\title{
Dual-Comb Modelocked Lasers
}

\section{Doctoral Thesis}

\section{Author(s):}

Link, Sandro

Publication date:

2017

Permanent link:

https://doi.org/10.3929/ethz-b-000199152

Rights / license:

In Copyright - Non-Commercial Use Permitted 
DISS. ETH NO. 24613

\title{
DUAL-COMB MODELOCKED LASERS
}

A thesis submitted to attain the degree of DOCTOR OF SCIENCES of ETH ZURICH

(Dr. sc. ETH Zurich)

\author{
presented by \\ Sandro Michael Link \\ MSc ETH Physics, ETH Zurich
}

born on 23.09.1987

citizen of Germany

accepted on the recommendation of

Prof. Dr. Ursula Keller, examiner Prof. Dr. Nathalie Picqué, co-examiner 

"Physics is really nothing more than a search for ultimate simplicity, but so far all we have is a kind of elegant messiness."

— Bill Bryson, A Short History of Nearly Everything 



\section{Contents}

Contents

List of figures vi vi vis

List of tables viii

$\begin{array}{ll}\text { List of symbols and acronyms } & \text { ix }\end{array}$

Publications $\quad$ xi

Abstract $\quad$ xix

Kurzfassung xxiii

1 Introduction $\quad 1$

2 Noise analysis of a MIXSEL 9

2.1 Noise characterization of a pulse train . . . . . . . . . . . 9

2.1 .1 Ideal pulse train . . . . . . . . . . . . . . 9

2.1.2 Amplitude noise ............... 10

2.1.3 Timing phase noise .............. . 11

2.2 Amplitude noise and timing jitter characterization of a highpower mode-locked integrated external-cavity surface emitting laser . . . . . . . . . . . . . . . . 13

2.2 .1 Introduction . . . . . . . . . . . . . 14

2.2.2 Laser design and mode-locking performance . . . . 16

2.2.3 Timing phase noise characterization and stabilization setup . . . . . . . . . . . . . . . . 19

2.2.4 Measured timing phase noise results . . . . . . . . 21

2.2 .5 Conclusion and outlook . . . . . . . . . . . . . 24

2.2.6 References.................. 24 
3 Dual-comb MIXSEL 29

3.1 Polarization duplexing . . . . . . . . . . . . . . . . . . . . . . . . $\quad 29$

3.2 Dual-comb modelocked laser . . . . . . . . . . . . . . . . 32

3.2 .1 Introduction . . . . . . . . . . . . . . . . 32

3.2 .2 Experimental setup . . . . . . . . . . . . . . . . 35

3.2 .3 Experimental results . . . . . . . . . . . . . . . . . . 38

3.2.4 Conclusion and outlook . . . . . . . . . . . . . . 45

3.2.5 References and links . . . . . . . . . . . . . . 45

4 Noise analysis of dual-comb modelocked lasers 51

4.1 Dual-comb modelocked lasers: semiconductor saturable absorber mirror decouples noise stabilization . . . . . . . . . 52

4.1.1 Introduction . . . . . . . . . . . . . 53

4.1.2 Phase noise of the dual-comb MIXSEL . . . . . . . . 56

4.1.3 Nd:YAG dual-comb . . . . . . . . . . . . . . . . . 60

4.1.4 Noise correlation study . . . . . . . . . . . . . . . 63

4.1.5 Conclusion and outlook . . . . . . . . . . . . . 70

4.1.6 References and links . . . . . . . . . . . . . . . 71

5 Dual-comb spectroscopy 77

5.1 Dual-comb spectroscopy principle . . . . . . . . . . . 78

5.2 Laser sources for dual-comb spectroscopy . . . . . . . . . 81

5.2.1 Quantum cascade laser (QCL) combs . . . . . . . . 82

5.2 .2 Microresonators . . . . . . . . . . . . . . 83

5.2.3 Further optical frequency comb (OFC) sources . . . 84

5.2.4 Dual-comb MIXSEL . . . . . . . . . . . . . . . . 84

5.3 Dual-comb spectroscopy of water vapor with a free-running semiconductor disk laser . . . . . . . . . . . . . . . 85

5.3.1 References and notes . . . . . . . . . . . . . . . 96

5.3.2 Supplementary materials . . . . . . . . . . . . . 98

6 Pulse shortening strategies for semiconductor disk lasers $\quad 103$

6.1 High-power $100 \mathrm{fs}$ semiconductor disk lasers . . . . . . . . . 104

6.1 .1 Introduction . . . . . . . . . . . . . . 105

6.1 .2 Laser design . . . . . . . . . . . . . . . . . 108

6.1 .3 Modelocking result . . . . . . . . . . . . . 111

6.1.4 Gain characterization . . . . . . . . . . . . . . . 115

6.1.5 Pulse formation simulation . . . . . . . . . . . . . 119

6.1.6 Performance scaling . . . . . . . . . . . . . . . . 121

6.1 .7 Conclusion . . . . . . . . . . . . . . . 122

6.1 .8 References. . . . . . . . . . . . . . . . . . . 123

7 Colliding pulse modelocked VECSELs 129

7.1 Coherent beam combining and noise analysis of a colliding pulse modelocked VECSEL . . . . . . . . . . . . . . 130

7.1 .1 Introduction . . . . . . . . . . . . . . . . 131

7.1 .2 Laser setup . . . . . . . . . . . . . . . . . . 132

7.1.3 Pulse repetition frequency analysis . . . . . . . . 134 
7.1.4 Carrier envelope offset (CEO) frequency analysis . . 136

7.1.5 Coherent beam combining . . . . . . . . . . 140

7.1.6 Conclusion and outlook . . . . . . . . . . . . . 143

7.1.7 References................... 144

8 Conclusion and outlook $\quad 147$

$\begin{array}{ll}\text { Literature } & 151\end{array}$

$\begin{array}{ll}\text { Curriculum Vitæ } & 155\end{array}$

$\begin{array}{lr}\text { Acknowledgments } & 157\end{array}$ 


\section{List of figures}

1.1 Frequency comb in the time and frequency domain . . . . . . . . . 2

1.2 Semiconductor disk laser configurations . . . . . . . . . . . . 5

2.1 Pulse train with amplitude noise . . . . . . . . . . . . . . . 11

2.2 Pulse train with timing phase noise . . . . . . . . . . . . . . . 12

2.3 MIXSEL design and housing . . . . . . . . . . . . . 17

2.4 Free-running MIXSEL modelocking results . . . . . . . . . . . . . . 18

2.5 Stabilized MIXSEL modelocking results . . . . . . . . . . . . . . . . . . 19

2.6 Schematic of noise characterization and stabilization . . . . . . . . . . 20

2.7 Noise characterization of MIXSEL . . . . . . . . . . . . . . . . . . 21

3.1 Beam propagation in birefringent crystal . . . . . . . . . . . . 31

3.2 Dual-comb MIXSEL gas sensing application . . . . . . . . . . . . . . . 35

3.3 Dual-comb MIXSEL pump-probe application . . . . . . . . . . . 36

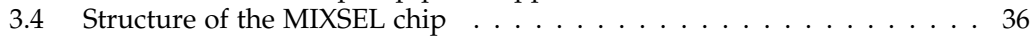

3.5 Dual-comb MIXSEL housing and setup . . . . . . . . . . . . . . 38

3.6 Modelocking results of the dual comb MIXSEL . . . . . . . . . . . . . . . 39

3.7 Microwave comb measurement and simulation . . . . . . . . . . . . . . . 42

3.8 Relative CEO frequency measurement and simulation . . . . . . . . . . . 43

3.9 Phase noise of pulse repetition rates of both beams . . . . . . . . . . . . . 44

$4.1 \quad$ Birefringent crystal for polarization duplexing . . . . . . . . . . . . . . 54

4.2 Dual-comb MIXSEL setup . . . . . . . . . . . . . . . . . 57

4.3 Noise characterization and stabilization setup . . . . . . . . . . . . . 58

4.4 Phase noise of the dual-comb MIXSEL . . . . . . . . . . . . . . . . . . . 59

4.5 Dual-comb Nd:YAG laser setup . . . . . . . . . . . . . . . . . . 61

4.6 Dual-comb Nd:YAG modelocking performance . . . . . . . . . . . . 62

4.7 Dual-comb Nd:YAG microwave spectrum . . . . . . . . . . . . 63

4.8 Phase noise of the dual-comb Nd:YAG . . . . . . . . . . . . . . . . . . . . 64

4.9 Beam separation with two birefringent crystals . . . . . . . . . . . 65

4.10 Dual-comb Nd:YAG microwave spectrum with two 1-mm-thick birefrin-

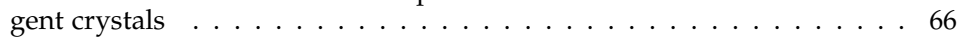

4.11 Dual-comb Nd:YAG microwave spectrum with two 3-mm-thick birefringent crystals ..................... 67 
4.12 Phase noise of the dual-comb MIXSEL with two 1-mm birefringent crys-

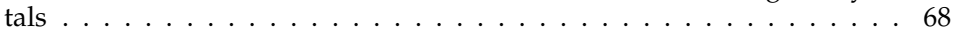

4.13 Pulse shift on saturable absorber and phase noise of the stabilized dualcomb MIXSEL . . . . . . . . . . . . . . . . . . . . . . . . 69

5.1 Gas spectroscopy principle with an optical frequency comb . . . . . . . . 77

5.2 Dual-comb frequency down-conversion principle . . . . . . . . . . . . 79

5.3 Dual-comb principle in the time domain . . . . . . . . . . . . . . . 80

5.4 Dual-comb gas spectroscopy concept . . . . . . . . . . . . . . . . 80

5.5 Current state-of-the-art performance in frequency comb characteristics . 87

5.6 Dual-comb source and operating principle . . . . . . . . . . . . . 88

5.7 Dual-comb spectroscopy results . . . . . . . . . . . . . . . . . . . . 91

5.8 Stabilization of the microwave comb and optical mode stability . . . . . 94

5.9 Second-harmonic autocorrelation of the two cross-polarized laser beams 98

5.10 Time-dependent interferogram . . . . . . . . . . . . . . . . . . . 99

5.11 Measurement setup for the absolute stability of the optical modes . . . 101

6.1 SDL pulse duration - peak power overview . . . . . . . . . . . . . 106

6.2 VECSEL cavity and chip design . . . . . . . . . . . . . . . . . . 109

6.3 Field intensity and GDD for VECSEL chip . . . . . . . . . . . . . . 111

$6.4128 \mathrm{fs}$ modelocking VECSEL result . . . . . . . . . . . . . . . . . 113

6.596 fs modelocking VECSEL result . . . . . . . . . . . . . . . . . . . 114

6.6 Spectral gain measurements . . . . . . . . . . . . . . . . . . 116

6.7 Gain saturation measurements . . . . . . . . . . . . . . . . . . . . . . . 118

6.8 Numerical pulse formation simulations . . . . . . . . . . . . . . . . . 120

7.1 Colliding pulse modelocked (CPM) VECSEL cavity . . . . . . . . . . . . . 132

7.2 CPM VECSEL modelocking results . . . . . . . . . . . . . . . . . . 134

7.3 Phase noise of the pulse repetition frequencies of the CPM VECSEL . . . 135

7.4 Analysis of the CEO frequencies of the CPM VECSEL . . . . . . . . . . 137

7.5 Long-term CEO frequency fluctuations of the CPM VECSEL . . . . . . 138

7.6 Coherent beam combining . . . . . . . . . . . . . . . . . 141

7.7 Intensity noise of combined beams . . . . . . . . . . . . . 142

8.1 Evolution of publications and citations about "dual-comb" over the last years . . . . . . . . . . . . . . . . . . 148 


\section{List of tables}

2.1 Timing-jitter comparison . . . . . . . . . . . . . . 23

3.1 Modelocking parameters of dual-comb pulse trains . . . . . . . . . 40

4.1 Overview of the different dual-comb laser configurations . . . . . . . . 64

6.1 Complete list of input parameters used for the pulse formation simulation112 


\section{List of symbols and acronyms}

\section{Symbols}

$\Delta f_{\text {lock }}$ bandwidth of locked frequencies for QCLs (Hz)

$\Delta f_{\text {CEO }}$ difference in carrier envelope offset frequency $(\mathrm{Hz})$

$\Delta f_{\text {rep }} \quad$ difference in pulse repetition frequency $(\mathrm{Hz})$

$f_{\text {CEO }} \quad$ carrier envelope offset frequency $(\mathrm{Hz})$

$f_{\text {rep }} \quad$ pulse repetition frequency $(\mathrm{Hz})$

$k_{n} \quad$ wavenumber $\left(\mathrm{m}^{-1}\right)$

$\delta v \quad$ optical laser linewidth $(\mathrm{Hz})$

$\Delta v \quad$ optical bandwidth $(\mathrm{Hz})$

$P_{\mathrm{av}} \quad$ average power $(\mathrm{W})$

$\sigma_{\mathrm{A}} \quad$ integrated amplitude noise (\%)

$\sigma_{\mathrm{T}} \quad$ integrated timing jitter (s)

$\tau_{\mathrm{p}} \quad$ pulse duration (FWHM) (s)

$T_{\mathrm{r}} \quad$ cavity roundtrip time (s)

$\rho \quad$ walk-off angle in birefringent crystal $\left({ }^{\circ}\right)$ 


\section{Acronyms}

ASOPS asynchronous optical sampling

CEO carrier envelope offset

CPM colliding pulse modelocking

$\mathrm{CW}$ continuous-wave

DBR distributed Bragg reflector

FWHM full-width at half maximum

FWM four-wave-mixing

GDD group delay dispersion

MIXSEL modelocked integrated external-cavity surface emitting laser

Nd:YAG neodymium-doped yttrium aluminium garnet $\left(\mathrm{Nd}: \mathrm{Y}_{3} \mathrm{Al}_{5} \mathrm{O}_{12}\right)$

OFC optical frequency comb

PSD power spectral density

QCL quantum cascade laser

rms root mean square

SDL semiconductor disk laser

SESAM semiconductor saturable absorber mirror

$\mathrm{TEM}_{00}$ fundamental transverse electromagnetic mode

VCSEL vertical cavity surface-emitting laser

VECSEL vertical external-cavity surface-emitting laser 


\section{Publications}

Parts of this thesis are published in the following journal papers and conference proceedings.

\section{Journal Papers}

1. C. G. E. Alfieri, D. Waldburger, S. M. Link, E. Gini, M. Golling, G. Eisenstein, U. Keller, "Optical efficiency and gain dynamics of modelocked semiconductor disk lasers", Opt. Express 6 (2017), 6402-6420

2. S. M. Link, D. J. H. C. Maas, D. Waldburger, U. Keller, "Dual-comb spectroscopy of water vapor with a free-running semiconductor disk laser", Science 356 (2017), 1164-1168

3. S. M. Link, D. Waldburger, C. G. E. Alfieri, M. Golling, U. Keller, "Coherent beam combining and noise analysis of a colliding pulse modelocked VECSEL", Opt. Express 25 (2017), 19281-19290

4. F. F. Voigt, F. Emaury, P. Bethge, D. Waldburger, S. M. Link, S. Carta, A. v. d. Bourg, M. Mangold, F. Helmchen, U. Keller, "Multiphoton in vivo imaging with a femtosecond semiconductor disk laser", Biomed. Opt. Express 8 (2017), 3213-3231

5. P. Brochard, N. Jornot, S. Schilt, V. Wittwer, S. Hakobyan, D. Waldburger, S. M. Link, C. Alfieri, M. Golling, L. Devenoges, J. Morel, U. Keller, T. Suedmeyer, "First Investigation of the Noise and Modulation Properties of the Carrier Envelope Offset in a Modelocked Semiconductor Laser", Opt. Lett. 41 (2016), 31653168

6. S. M. Link, A. Klenner, U. Keller, "Dual-comb modelocked lasers: semiconductor saturable absorber mirror decouples noise stabilization", Opt. Express 24 (2016), 1889-1902

7. S. M. Link, M. Mangold, M. Golling, A. Klenner, U. Keller, "Dual-comb modelocked lasers", SPIE Newsroom (2016), invited 
8. D. Waldburger, S. M. Link, M. Mangold, C. G. E. Alfieri, E. Gini, M. Golling, B. W. Tilma, U. Keller, "High-power 100 fs semiconductor disk lasers", Optica 3 (2016), 844-852

9. S. M. Link, A. Klenner, M. Mangold, C. A. Zaugg, M. Golling, B. W. Tilma, U. Keller, "Dual-comb modelocked laser", Opt. Express 23 (2015), 5521-5531

10. B. W. Tilma, M. Mangold, C. A. Zaugg, S. M. Link, D. Waldburger, A. Klenner, A. S. Mayer, E. Gini, M. Golling, U. Keller, "Recent advances in ultrafast semiconductor disk lasers", Light Sci Appl 4 (2015), e310, invited

11. M. Mangold, S. M. Link, A. Klenner, C. A. Zaugg, M. Golling, B. W. Tilma, U. Keller, "Amplitude noise and timing jitter characterization of a high-power mode-locked integrated external-cavity surface emitting laser", IEEE Photonics Journal 6 (2014), 1-9

12. M. Mangold, C. A. Zaugg, S. M. Link, M. Golling, B. W. Tilma, U. Keller, "Pulse repetition rate scaling from $5 \mathrm{GHz}$ to $100 \mathrm{GHz}$ with a high-power semiconductor disk laser", Optics Express 22 (2014), 6099-6107

13. C. Schriber, F. Emaury, A. Diebold, S. M. Link, M. Golling, K. Beil, C. Kraenkel, C. J. Saraceno, T. Suedmeyer, U. Keller, "Dual-gain SESAM modelocked thin disk laser based on Yb:Lu2O3 and Yb:Sc2O3", Opt. Express 22 (2014), 1897918986

14. C. A. Zaugg, A. Klenner, M. Mangold, A. S. Mayer, S. M. Link, F. Emaury, M. Golling, E. Gini, C. J. Saraceno, B. W. Tilma, U. Keller, "Gigahertz selfreferencable frequency comb from a semiconductor disk laser", Optics Express 22 (2014), 16445-16455

15. M. Mangold, V. J. Wittwer, C. A. Zaugg, S. M. Link, M. Golling, B. W. Tilma, U. Keller, "Femtosecond pulses from a modelocked integrated external-cavity surface emitting laser (MIXSEL)", Optics Express 21 (2013), 24904-24911

\section{Patent}

1. S. M. Link, B. W. Tilma, M. Mangold, C. A. Zaugg, A. Klenner, U. Keller, "Pulsed laser", International Patent, WO 2016/049787 A1 (filed September 31, 2014, issued April 7, 2016) 


\section{Conference Papers}

1. F. Emaury, F. F. Voigt, P. Bethge, D. Waldburger, S. M. Link, S. Carta, A. v. d. Bourg, F. Helmchen, U. Keller, "Femtosecond semiconductor disk lasers: a promising tool for the future of multiphoton imaging", in SPIE Photonics West - BIOS, San Francisco, USA (2018), submitted

2. J. Nürnberg, S. M. Link, C. G. E. Alfieri, D. Waldburger, M. Golling, U. Keller, "Femtosecond dual-comb MIXSEL at $1030 \mathrm{~nm}$ ", in SPIE Photonics West - LASE, San Francisco, USA (2018), submitted

3. D. Waldburger, S. M. Link, C. G. E. Alfieri, M. Golling, U. Keller, "Coherent beam combining of a colliding pulse modelocked VECSEL", in SPIE Photonics West - LASE, San Francisco, USA (2018), submitted

4. C. G. E. Alfieri, D. Waldburger, S. M. Link, M. Golling, U. Keller, "Carrier dynamics of ultrafast semiconductor disk lasers", in Conference on Lasers and Electro-Optics (CLEO), San Jose, USA (2017), poster

5. C. G. E. Alfieri, D. Waldburger, S. M. Link, M. Golling, U. Keller, "Optical efficiency and gain dynamics of semiconductor disk lasers", in SPIE Photonics West - LASE, San Francisco, USA (2017), invited talk

6. C. G. E. Alfieri, D. Waldburger, S. M. Link, M. Golling, U. Keller, “Ultrafast dynamics of semiconductor disk lasers", in Conference on Lasers and ElectroOptics (CLEO)-EQEC, Munich, Germany (2017), talk

7. F. Emaury, F. F. Voigt, P. Bethge, D. Waldburger, S. M. Link, S. Carta, A. v. d. Bourg, F. Helmchen, U. Keller, "Multiphoton microscopy in every lab: the promise of ultrafast semiconductor disk lasers", in Conference on Lasers and Electro-Optics (CLEO)-ECBO, Munich, Germany (2017), talk

8. F. Emaury, F. F. Voigt, P. Bethge, D. Waldburger, S. M. Link, S. Carta, F. Helmchen, U. Keller, "Ultrafast semiconductor disk lasers for in-vivo multi-photon microscopy", in Conference on Lasers and Electro-Optics (CLEO), San Jose, USA (2017), talk

9. S. M. Link, D. J. H. C. Maas, D. Waldburger, C. G. E. Alfieri, M. Golling, F. Emaury, U. Keller, "Gas spectroscopy with a dual-comb semiconductor disk laser", in Conference on Lasers and Electro-Optics (CLEO), San Jose, USA (2017), talk

10. S. M. Link, D. J. H. C. Maas, D. Waldburger, U. Keller, "Gas spectroscopy with a free-running dual-comb semiconductor disk laser", in Conference on Lasers and Electro-Optics (CLEO)-EQEC, Munich, Germany (2017), talk

11. S. M. Link, D. Waldburger, C. G. E. Alfieri, M. Golling, U. Keller, "Stabilized dual-comb MIXSEL", in SPIE Photonics West - LASE, San Francisco, USA (2017), invited talk

12. D. J. H. C. Maas, S. M. Link, D. Waldburger, U. Keller, "Dual-comb spectroscopy with a semiconductor disk laser", in IEEE Sensors 2017, Glasgow, Scotland (2017), talk 
13. F. F. Voigt, F. Emaury, P. Bethge, D. Waldburger, S. M. Link, S. Carta, A. v. d. Bourg, F. Helmchen, U. Keller, "In vivo multiphoton imaging using ultrafast semiconductor disk lasers", in Focus on Microscopy (FoM), Bordeaux, France (2017), poster

14. D. Waldburger, C. G. E. Alfieri, S. M. Link, E. Gini, M. Golling, U. Keller, "Gigahertz 100-fs semiconductor disk lasers", in Compound Semiconductor Week, Berlin, Germany (2017), talk

15. D. Waldburger, C. G. E. Alfieri, S. M. Link, E. Gini, M. Golling, U. Keller, "Highpower semiconductor disk lasers with record-short pulse durations", in Conference on Lasers and Electro-Optics (CLEO)-EQEC, Munich, Germany (2017), talk

16. D. Waldburger, S. M. Link, C. G. E. Alfieri, M. Golling, U. Keller, "Coherent beam combining of a colliding pulse modelocked VECSEL", in Advanced SolidState Lasers (ASSL), Nagoya, Japan (2017), talk

17. D. Waldburger, S. M. Link, C. G. E. Alfieri, M. Golling, U. Keller, "High-power 100-fs SESAM modelocked VESCEL", in SPIE Photonics West - LASE, San Francisco, USA (2017), invited talk

18. D. Waldburger, S. M. Link, D. J. H. C. Maas, U. Keller, "Free-running dual-comb MIXSEL used for dual-comb spectroscopy", in Advanced Solid-State Lasers (ASSL), Nagoya, Japan (2017), talk

19. C. G. E. Alfieri, D. Waldburger, S. M. Link, E. Gini, M. Golling, B. W. Tilma, M. Mangold, U. Keller, "Recent progress in high power ultrafast MIXSELs", in SPIE Photonics West - LASE, San Francisco, USA (2016), talk

20. P. Brochard, N. Jornod, V. Wittwer, S. Schilt, D. Waldburger, S. M. Link, C. G. E. Alfieri, M. Golling, L. Devenoges, J. Morel, U. Keller, T. Südmeyer, "Carrierenvelope offset characterization in a semiconductor modelocked laser without f-to-2f interferometry", in European Frequency and Time Forum (EFTF), York, United Kingdom (2016), talk

21. M. Golling, C. G. E. Alfieri, D. Waldburger, S. M. Link, E. Gini, U. Keller, "Progress in MBE and MOVPE semiconductor disk laser growth", in Deutscher MBE Workshop, Munich, Germany (2016), talk

22. N. Jornod, P. Brochard, V. Wittwer, S. Schilt, S. Hakobyan, D. Waldburger, S. M. Link, C. G. E. Alfieri, M. Golling, L. Devenoges, J. Morel, U. Keller, T. Südmeyer, "First Investigation of the noise and modulation properties of the carrier envelope offset frequency in a semiconductor modelocked laser", in Conference on Lasers and Electro-Optics (CLEO), San Jose, USA (2016), talk

23. S. M. Link, C. G. E. Alfieri, D. Waldburger, A. Klenner, M. Golling, U. Keller, "Saturable absorber decouples noise stabilization in dual-comb modelocked lasers", in Conference on Lasers and Electro-Optics (CLEO), San Jose, USA (2016), talk

24. S. M. Link, M. Mangold, M. Golling, A. Klenner, U. Keller, "Gigahertz dualcomb modelocked diode-pumped semiconductor and solid-state lasers", in SPIE Photonics West - LASE, San Francisco, USA (2016), invited talk 
25. S. M. Link, D. Waldburger, C. G. E. Alfieri, M. Golling, E. Gini, U. Keller, "Record-short pulses from semiconductor disk lasers", in $7^{\text {th }}$ EPS-QEOD Europhoton Conference, Vienna, Austria (2016), talk

26. S. M. Link, D. Waldburger, C. G. E. Alfieri, M. Golling, U. Keller, “Stabilized dual-comb modelocked semiconductor disk laser", in $7^{\text {th }}$ EPS-QEOD Europhoton Conference, Vienna, Austria (2016), post-deadline talk

27. S. M. Link, D. Waldburger, C. G. E. Alfieri, M. Golling, U. Keller, "Stabilized microwave frequency comb from a dual-comb modelocked semiconductor disk laser", in Advanced Solid-State Lasers (ASSL), Boston, USA (2016), talk

28. D. Waldburger, C. G. E. Alfieri, S. M. Link, E. Gini, M. Golling, M. Mangold, B. W. Tilma, U. Keller, "Pulse shortening of an ultrafast VECSEL", in SPIE Photonics West - LASE, San Francisco, USA (2016), talk

29. D. Waldburger, S. M. Link, C. G. E. Alfieri, M. Golling, U. Keller, "High-power 100-fs SESAM-modelocked VECSEL", in Advanced Solid-State Lasers (ASSL), Boston, USA (2016), talk

30. D. Waldburger, S. M. Link, C. G. E. Alfieri, M. Golling, U. Keller, "Multi-pulse behavior of a high-power sub-100-fs SESAM-modelocked VECSEL", in $7^{\text {th }}$ EPSQEOD Europhoton Conference, Vienna, Austria (2016), poster

31. C. G. E. Alfieri, D. Waldburger, S. M. Link, E. Gini, M. Mangold, B. W. Tilma, M. Golling, U. Keller, "Recent progress in high-power femtosecond semiconductor disk lasers", in Advanced Solid-State Lasers (ASSL), Berlin, Germany (2015), talk

32. M. Golling, M. Mangold, C. A. Zaugg, S. M. Link, D. Waldburger, C. G. E. Alfieri, B. W. Tilma, U. Keller, "MBE growth of semiconductor disk laser for repetition rate scaling from 5 to $100 \mathrm{GHz}^{\prime}$, in 18th European Molecular Beam Epitaxy Workshop (EUROMBE), Canazei, Italy (2015), talk

33. S. M. Link, M. Mangold, M. Golling, A. Klenner, U. Keller, "Modelocked dualcomb lasers", in Advanced Solid-State Lasers (ASSL), Berlin, Germany (2015), talk

34. S. M. Link, M. Mangold, M. Golling, B. W. Tilma, U. Keller, "Dual-comb modelocked lasers", in Conference on Lasers and Electro-Optics (CLEO), San Jose, USA (2015), talk

35. S. M. Link, M. Mangold, M. Golling, B. W. Tilma, U. Keller, "Dual-comb modelocked lasers", in Conference on Lasers and Electro-Optics (CLEO)-EQEC, Munich, Germany (2015), talk

36. S. M. Link, C. A. Zaugg, A. Klenner, M. Mangold, M. Golling, B. W. Tilma, U. Keller, "Dual-comb MIXSEL", in SPIE Photonics West - LASE, San Francisco, USA (2015), talk

37. M. Mangold, D. Waldburger, S. M. Link, M. Golling, E. Gini, B. W. Tilma, U. Keller, "High-power sub-300-femtosecond semiconductor disk lasers", in Conference on Lasers and Electro-Optics (CLEO)-EQEC, Munich, Germany (2015), upgrade to invited talk 
38. M. Mangold, C. A. Zaugg, A. Klenner, A. S. Mayer, S. M. Link, F. Emaury, M. Golling, E. Gini, C. J. Saraceno, B. W. Tilma, U. Keller, "Carrier-envelopeoffset frequency detection of an ultrafast VECSEL", in SPIE Photonics West LASE, San Francisco, USA (2015), invited talk

39. D. Waldburger, M. Mangold, S. M. Link, M. Golling, E. Gini, B. W. Tilma, U. Keller, "Sub-300-femtosecond semiconductor disk lasers", in Conference on Lasers and Electro-Optics (CLEO), San Jose, USA (2015), talk

40. M. Golling, M. Mangold, C. A. Zaugg, S. M. Link, B. W. Tilma, U. Keller, "Wachstum von optisch gepumpten vertikal-emittierenden Scheibenlasern und SESAMs in der MBE", in Deutscher MBE Workshop, Darmstadt, Germany (2014), talk

41. U. Keller, M. Mangold, S. M. Link, C. A. Zaugg, A. Klenner, A. S. Mayer, M. Golling, B. W. Tilma, "MIXSEL: a novel ultrafast semiconductor laser", in $45^{\text {th }}$ Winter Colloquium on the Physics of Quantum Electronics, Snowbird, USA (2014), invited talk

42. U. Keller, M. Mangold, C. A. Zaugg, S. M. Link, A. Klenner, A. S. Mayer, M. Golling, B. W. Tilma, "Ultrafast semiconductor lasers", in $24^{\text {th }}$ International Semiconductor Laser Conference (ISLC), Palma de Mallorca, Spain (2014), invited talk

43. A. Klenner, C. A. Zaugg, M. Mangold, A. S. Mayer, S. M. Link, F. Emaury, M. Golling, E. Gini, C. J. Saraceno, B. Tilma, S. Schilt, T. Suedmeyer, U. Keller, "Diode-pumped gigahertz frequency combs", in Advanced Solid-State Lasers (ASSL), Shanghai, China (2014), talk

44. S. M. Link, A. Klenner, M. Mangold, C. A. Zaugg, A. S. Mayer, E. Gini, B. W. Tilma, U. Keller, "Passive linewidth reduction of the carrier-envelope-offset frequency through high-brightness pumping", in $6^{\text {th }}$ EPS-QEOD Europhoton Conference, Neuchatel, Switzerland (2014), poster

45. S. M. Link, M. Mangold, V. J. Wittwer, A. Klenner, M. Golling, B. W. Tilma, U. Keller, "Record-low noise performance of high-power picosecond MIXSEL", in SPIE Photonics West - LASE, San Francisco, USA (2014), talk

46. M. Mangold, S. M. Link, C. A. Zaugg, A. Klenner, A. S. Mayer, M. Golling, B. W. Tilma, U. Keller, "High pulse repetition rate and low noise MIXSEL", in Advanced Solid-State Lasers (ASSL), Shanghai, China (2014), talk

47. M. Mangold, V. J. Wittwer, C. A. Zaugg, S. M. Link, M. Golling, B. W. Tilma, U. Keller, "Femtosecond MIXSEL", in SPIE Photonics West - LASE, San Francisco, USA (2014), invited talk

48. M. Mangold, V. J. Wittwer, C. A. Zaugg, S. M. Link, M. Golling, B. W. Tilma, U. Keller, "Picosecond MIXSEL for clocking applications", in SPIE Photonics West - LASE, San Francisco, USA (2014), talk

49. M. Mangold, C. A. Zaugg, S. Link, A. Klenner, A. S. Mayer, M. Golling, B. W. Tilma, U. Keller, "Recent advances in ultrafast MIXSELs", in $6^{\text {th }}$ EPS-QEOD Europhoton Conference, Neuchatel, Switzerland (2014), talk 
50. M. Mangold, C. A. Zaugg, S. M. Link, A. Klenner, M. Golling, B. W. Tilma, U. Keller, "Recent advances in ultrafast MIXSELs", in Conference on Lasers and Electro-Optics (CLEO), San Jose, USA (2014), talk

51. C. A. Zaugg, A. Klenner, M. Mangold, A. S. Mayer, S. M. Link, F. Emaury, M. Golling, E. Gini, C. J. Saraceno, B. W. Tilma, U. Keller, "Supercontinuum generation from a 1.75-GHz SESAM modelocked VECSEL and carrier envelope offset frequency detection", in $6^{\text {th }}$ EPS-QEOD Europhoton Conference, Neuchatel, Switzerland (2014), talk

52. U. Keller, M. Mangold, V. J. Wittwer, C. A. Zaugg, S. M. Link, M. Golling, B. W. Tilma, "High power ultrafast VECSELs and MIXSELs", in Fundamental optical processes in semiconductors (FOPS), Kodiak Island, Alaska, USA (2013), invited talk

53. M. Mangold, S. M. Link, V. J. Wittwer, A. Klenner, M. Golling, B. W. Tilma, U. Keller, "Record low timing jitter of a free-running high-power MIXSEL", in Advanced Solid-State Lasers (ASSL), Paris, France (2013), poster

54. M. Mangold, C. A. Zaugg, V. J. Wittwer, S. M. Link, M. Golling, B. W. Tilma, U. Keller, "MIXSEL generates femtosecond pulses and tunable gigahertz pulse repetition rates from 5-100 GHz", in Advanced Solid-State Lasers (ASSL), Paris, France (2013), post-deadline talk

55. B. W. Tilma, C. A. Zaugg, M. Mangold, S. M. Link, A. Klenner, M. Golling, U. Keller, "High power ultrafast VECSELs and MIXSELs", in $18^{\text {th }}$ Annual Symposium of the IEEE Photonics Benelux Chapter, Eindhoven, Netherlands (2013), invited talk

56. C. A. Zaugg, M. Mangold, S. M. Link, V. J. Wittwer, A. Klenner, M. Golling, B. W. Tilma, U. Keller, "Recent advances in ultrafast MIXSELs", in $2^{\text {nd }}$ Workshop VeCSEL, Montpellier, France (2013), talk 



\section{Abstract}

Modelocked lasers emit pulse trains of ultrashort light pulses with durations of only a few pico- $\left(1 \mathrm{ps}=10^{-12} \mathrm{~s}\right)$ or even femtoseconds $(1 \mathrm{fs}=$ $10^{-15} \mathrm{~s}$ ), allowing access to previously unresolvably fast dynamics. At the same time, their optical spectrum consists of a large number of equidistantly spaced frequency-lines with a relative uncertainty down to $10^{-19}$. These so-called optical frequency combs (OFCs) enabled a whole new scientific field - the frequency metrology - with myriads of emerging applications. The widespread use of these applications and further growth of the field demands new, simple, compact, and cost-efficient laser sources. In particular, advanced concepts which rely on two OFCs would greatly benefit from these improved sources. One example for these concepts is dual-comb spectroscopy, which is the targeted application of this thesis. Dual-comb spectroscopy enables non-invasive molecular spectroscopy with unprecedented speed and accuracy.

Lasers based on the semiconductor technology have shown that they are an ideal choice when it comes to compactness, miniaturization and price. Besides their economical benefits they also have demonstrated a tremendous performance progress since their first demonstration in 1962. In particular with semiconductor disk lasers (SDLs), also referred to as vertical external-cavity surface-emitting lasers (VECSELs), remarkable results have been displayed. Their thin gain structure and the vertical emission allow for efficient heat removal and mode-size scaling, facilitating high-power operation with more than $100 \mathrm{~W}$ in continuous-wave (cw) 
operation. In combination with semiconductor saturable absorber mirrors (SESAMs) inside a linear cavity, modelocking with pulse durations below $100 \mathrm{fs}$ is possible, as presented within this thesis. We also show that in case VECSEL and SESAM are used in a ring cavity for colliding pulse modelocking, the two output beams can be coherently combined for power scaling. Very small cavities can be designed with SDLs and pulse repetition rates ranging from $100 \mathrm{MHz}$ to $100 \mathrm{GHz}$ have been presented. The short upper-state lifetime and the high gain cross-section prevent semiconductor lasers from Q-switching instabilities, a common issue for high-repetition rate solid-state lasers.

The level of integration of these lasers can be further increased by combing the saturable absorber of the SESAM with the gain structure of a VECSEL in a single semiconductor chip. These modelocked integrated external-cavity surface emitting lasers (MIXSELs) allow for modelocking in a straight linear cavity. Inserting a birefringent crystal into this simple cavity leads to the simultaneous emission of two cross-polarized fundamentally modelocked beams with slightly different pulse repetition rates, i.e. two OFCs with a slightly different comb-line spacing. This dualcomb MIXSEL represents a smart and powerful source for dual-comb spectroscopy and other applications relying on two OFCs and is demonstrated for the first time within this thesis. The noise is characterized in detail and perturbations are stabilized for a single and a dual-comb MIXSEL. Its superb noise performance, with e.g. relative comb-line spacing variation of $\approx 2.5 \times 10^{-4}$ integrated over $10 \mathrm{~ms}$ in free-running operation, qualifies the MIXSEL for frequency metrology applications. In a first proof-of-principle dual-comb spectroscopy experiment the absorption of water vapor is detected with a dual-comb MIXSEL. The high mutual short-term coherence of the two beams due to the common laser cavity enabled absorption measurements with excellent agreement with the HITRAN database even in free-running operation. For long integration times, a simple yet effective stabilization scheme is presented that relies only on microwave signals and circumvents more complex optical stabilization methods.

The emission wavelength of SDLs can be tailored with band-gap engineering. This wavelength flexibility is a unique feature of semiconductor lasers and another advantage of the dual-comb MIXSEL, allowing the de- 
tection of different gas species, further underlining its potential as attractive, compact and affordable source for dual-comb spectroscopy. 



\section{Kurzfassung}

Modengekoppelte Laser emittieren Pulszüge aus ultrakurzen Lichtpulsen, die nur wenige Piko- $\left(1 \mathrm{ps}=10^{-12} \mathrm{~s}\right)$ oder sogar Femtosekunden $(1 \mathrm{fs}=$ $10^{-15} \mathrm{~s}$ ) dauern und Zugriff auf bisher unauflösbar schnelle Dynamiken ermöglichen. Zudem besteht ihr optisches Spektrum aus einer großen Anzahl an Frequenzlinien mit äquidistantem Abstand und einer möglichen relativen Ungenauigkeit die auf bis zu $10^{-19}$ reduziert werden kann. Diese so genannten optischen Frequenzkämme erschlossen ein gänzlich neues Gebiet der Wissenschaft, die Frequenzmetrologie, mit unzähligen entstehenden Anwendungen. Für die flächendeckende Verwendung dieser Anwendungen und das weitere Wachstum dieses Gebiets werden neue, einfache, kompakte und kosteneffiziente Laserquellen benötigt. Insbesondere fortgeschrittene Konzepte, die auf zwei dieser Frequenzkämme basieren, würden außerordentlich von solch neuen Quellen profitieren. Ein Beispiel für diese Konzepte ist Doppel-Kamm Spektroskopie, welches die in dieser Doktorarbeit anvisierte Anwendung ist. Doppel-Kamm Spektroskopie ermöglicht nicht-invasive molekulare Spektroskopie mit bisher unerreichter Messgeschwindigkeit und Präzision.

Eine ideale Wahl bezüglich Kompaktheit, Miniaturisierung und Preis sind Laser die auf Halbleitertechnologie basieren. Neben ihren ökonomischen Vorteilen zeigten sie auch einen enormen Fortschritt bezüglich ihrer Leistungsfähigkeit seit der ersten Demonstration im Jahr 1962. Insbesondere mit Halbleiterscheibenlasern (engl. semiconductor disk lasers (SDLs) oder vertical external-cavity surface-emit-ting lasers (VEC- 
SELs)) wurden beeindruckende Ergebnisse erreicht. Durch die dünne Verstärkunsschicht und die Emission in vertikaler Richtung wird eine effiziente Wärmeableitung und Skalierung der Lasermodengröße gewährleistet, was hohe Durchschnittsleistungen mit mehr als $100 \mathrm{~W}$ im Dauerstrich-Betrieb ermöglicht. Die Kombination mit sättigbaren Halbleiterspiegeln (engl. semiconductor saturable absorber mirror (SESAM)) in einem linearen Resonator erlaubt Modenkopplung mit Pulslängen von weniger als $100 \mathrm{fs}$, was in dieser Doktorarbeit präsentiert wird. Für den Fall dass VECSEL und SESAM in einem Ring Resonator für Modenkopplung mit kollidierenden Pulsen verwendet werden, zeigen wir dass beide Ausgangsstrahlen kohärent kombiniert werden können um Leistungsskalierbarkeit zu erreichen. Mit Halbleiterscheibenlasern können sehr kurze Resonatorlängen konzipiert werden, was zu Pulswiederholraten zwischen $100 \mathrm{MHz}$ und $100 \mathrm{GHz}$ führt. Die kurze Lebensdauer des angeregten Zustands und der hohe Verstärkungswirkungsquerschnitt verhindern bei Halbleiterlasern das Auftreten von Instabilitäten aufgrund von Güteschaltung, welches ein allgemeines Problem für Festkörper Laser mit hohen Pulswiederholraten darstellt.

Durch die Kombination des sättigbaren Absorbers des SESAMs und der Verstärkungsstruktur eines VECSELs in einem einzigen Halbleiterchip kann der Integrationsgrad dieser Laser weiter erhöht werden. Diese Klasse von Lasern wird als MIXSEL (engl. modelocked integrated external-cavity surface-emitting laser) bezeichnet und ermöglicht Modenkopplung in einem geraden linearen Laserresonator. Das Einfügen eines doppelbrechenden Kristalls in diesen einfachen Resonator führt zur gleichzeitigen Emission zweier fundamental modengekoppelter Strahlen mit leicht unterschiedlichen Pulswiederholungsraten und senkrecht zueinander stehenden Polarisationen. Dieses Doppel-Kamm MIXSEL wird in dieser Doktorarbeit zum ersten Mal demonstriert und stellt eine elegante und leistungsfähige Quelle dar für Doppel-Kamm Spektroskopie und andere Anwendungen, die auf zwei optischen Frequenzkämmen basieren. Das Rauschverhalten wird detailliert charakterisiert und Störungen stabilisiert sowohl für ein einfaches als auch für ein Doppel-Kamm MIXSEL. Dank seines hervorragenden Rauschverhaltens, mit beispielsweise einer relativen Unsicherheit des Kammlinienabstands von nur $\approx 2.5 \times 10^{-4}$ integriert über $10 \mathrm{~ms}$ ohne 
aktive Stabilisierung, eignet sich das MIXSEL bestens für Anwendungen im Bereich der Frequenzmetrologie. In der weltweit ersten Demonstration detektieren wir die Absorption von Wasserdampf mit dem Doppel-Kamm MIXSEL. Durch den gemeinsamen Laserresonator ermöglicht die hohe gegenseitige Kurzzeit-Kohärenz der zwei Strahlen Absorptionsmessungen mit exzellenter Übereinstimmung mit der HITRAN Datenbank sogar ohne aktive Stabilisierung. Für Messungen mit langen Integrationszeiten wird ein geschicktes Stabilisierungsschema präsentiert, welches nur auf Mikrowellen-Signalen basiert und komplexere optische Stabilisierungsmethoden umgeht.

Die Emissionswellenlänge von Halbleiterscheibenlasern kann durch die spezifische Anpassung der Bandlücke des Halbleitermaterials maßgeschneidert werden. Diese Flexibilität der Wellenlänge ist eine einzigartige Fähigkeit von Halbleiterlasern und ein weiterer Vorteil des Doppel-Kamm MIXSELs, der die Messung verschiedener Gasspezien erlaubt und der das Potential als attraktive, kompakte und bezahlbare Quelle für DoppelKamm Spektroskopie untermauert. 



\section{Chapter 1}

\section{Introduction}

The pursuit of controlling and utilizing light has always been fundamental for humankind. Achieving new ways of generating light marked new chapters in human history. The control of fire hundred thousands of years ago gave the early humans a decisive advantage and was a key factor for the evolution of the human species. The commercial manufacturing of light bulbs in 1880 through Thomas Edison and the following electrical light started a new era.

When the first laser was demonstrated by Theodor Maiman in 1960 [1], its impact was not foreseen and it was even considered a solution looking for a problem. Today, we know that lasers revolutionized our technology in countless fields. To just mention a few, lasers have changed the way we communicate, process materials, store data, treat patients and measure precisely an enormous variety of parameters. The digital age of our modern society could not be imagined without lasers.

A laser emits coherent light, possibly with a single frequency in continuous-wave (cw) operation, or with a broad optical spectrum if the light is generated in extremely short packages of light. The duration of such a light pulse can be as short as a few pico- $\left(10^{-12} \mathrm{~s}\right)$ or even femtoseconds $\left(10^{-15} \mathrm{~s}\right)$. These short pulse durations are approaching timescales of fundamental atomic and molecular processes, allowing observations and manipulations with unprecedented time resolution.

But ultra-short laser pulses are not only interesting from their temporal 
aspect. A train of evenly spaced short pulses in time is inherently linked to a comb of equidistantly spaced optical frequencies (Fig. 1.1). The transition from the time domain to the frequency domain and vice versa is described by the fundamental principle of Fourier transformation (FT). The optical spectrum of a pulse train emitted by a modelocked laser is
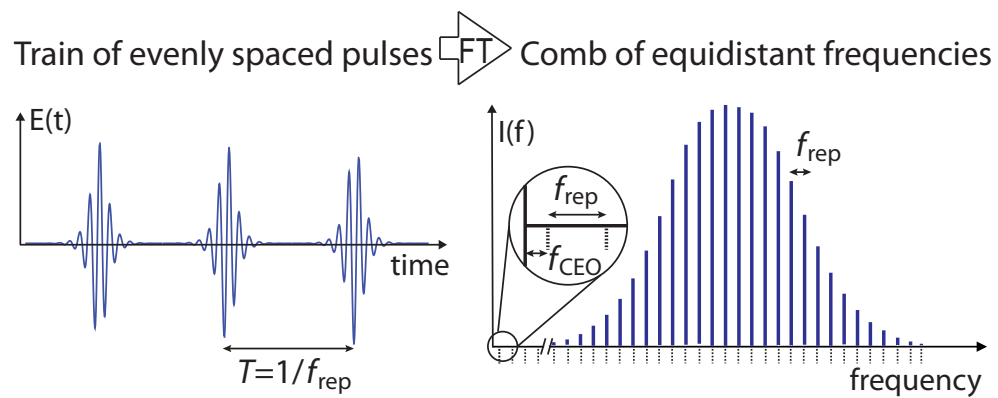

Figure 1.1: A train of evenly spaced pulses in time represents an optical frequency comb (OFC) with equidistant frequencies in the frequency domain. The transition between time and frequency domain is given by the principle of Fourier transformation (FT). The spacing of the comb-lines $f_{\text {rep }}$ is defined as one over the separation of the pulses in time. The absolut position of the optical frequency comb (OFC) is additionally determined by its offset to zero, the so called carrier envelope offset (CEO) frequency.

such an OFC. The whole comb is defined by only two frequencies:

- the spacing of the comb lines, called the pulse repetition frequency or pulse repetition rate, which is set by the time between two pulses $T$ via $T=\frac{1}{f_{\text {rep }}}$

- The offset of the comb to zero, called the carrier envelope offset (CEO) frequency

Once these two frequencies are well known or stabilized against perturbations, the OFC acts as an extremely precise frequency ruler. This very versatile tool has enabled the new field of optical metrology and was even recognized with the Nobel prize in 2005 [2].

An unknown frequency can be measured by generating a beat frequency between the unknown frequency and the OFC. This beat frequency 
is in the microwave regime and can be directly measured. The OFC creates a direct link between the optical (several hundreds of $\mathrm{THz}$ ) frequencies and the electronically accessible microwave $(\mathrm{MHz})$ frequencies. The OFC can not only interact with light but also with matter, for example a gas. The specific electronic or ro-vibrational transitions of a gas lead to absorption of the light, resulting in a modulation of the amplitude of the individual frequencies of the comb. If the OFC is measured after the interaction with the medium and the frequencies can be resolved individually, the gas species and its concentration can be determined. The challenge is the detection of these optical frequencies, since they are too fast to be measured with available electronics. One very attractive solution is the use of a second OFC with slightly different comb-line spacing. The principle is similar to measuring an unknown frequency with an OFC, but instead of measuring a single frequency, all frequencies of the comb are down-converted simultaneously (see chapter 5). This technique was for the first time proposed in 2002 [3] and is called dual-comb spectroscopy. It offers non-invasive measurements of gases or liquids with extremely high precision and acquisition speed. Since the first realization in 2004 [4], the field has attracted a lot of attention and the interest of a broad scientific community [5].

Currently, the main limitation of this technique is the complexity of the laser source. Usually, two modelocked Ti:Sapphire or fiber lasers are used. In order to fully stabilize both lasers to achieve a well resolved signal, they need to be nonlinearly broadened until their optical spectra span an octave and the $\mathrm{CEO}$ frequencies can be detected and stabilized via f-to- $2 \mathrm{f}$ interferometry [6]. These systems can deliver remarkable results, however, their complexity and very high price is hindering a more widespread usage for most applications outside of the research laboratory.

The main emphasis of this thesis is the development of a simple, compact, and cost-efficient source for dual-comb spectroscopy.

Lasers based on semiconductor gain materials are ideal candidates for small and low-priced laser sources. The first semiconductor laser diode was presented in 1962 [7], just two years after the invention of the laser. For a long time, these devices were all based on electrically pumped edge- 
emitting structures. Due to their very small foot-print and small price, they became extremely popular. However, they are not well suited for applications that require a high brightness and a good beam quality. To overcome these limitations, new concepts with vertical emission instead of edge emission were proposed, with a first demonstration in 1979 in form of a vertical cavity surface-emitting laser (VCSEL) [8]. This electrically pumped monolithic laser is widely used for example in the field of optical communication. It can be found in nearly every household within computer mice. However, for several applications it is beneficial to have an external cavity instead of a monolithic approach, for instance to generate short pulses in a modelocked laser. The external cavity was realized in 1993 [9], but the electrically pumped devices were limited in achievable output power. Consequently, the first optically pumped vertical externalcavity surface-emitting laser (VECSEL), also referred to as semiconductor disk laser (SDL), was presented in 1997 [10]. These devices combine a great number of advantages :

- wafer-scale fabrication enables cost-efficient mass-production

- very compact laser cavity

- semiconductor band-gap engineering allows to taylor the wavelength and enables a large spectral coverage from the visible up to the mid-infrared $[11,12,13,14,15,16]$

- the power is scalable with the mode area

- high power operation is further supported through efficient heat removal due to the one-dimensional heat flow

- high gain cross-section

- perfect beam quality with fundamental transverse electromagnetic mode $\left(\mathrm{TEM}_{00}\right)$ operation thanks to external mode control and choice of pump-spot-size

- short interaction length with gain medium leads to low intracavity nonlinearities

- the high-Q external cavity enables low noise performance and a variety of different laser operations such as intracavity frequency doubling and modelocking 


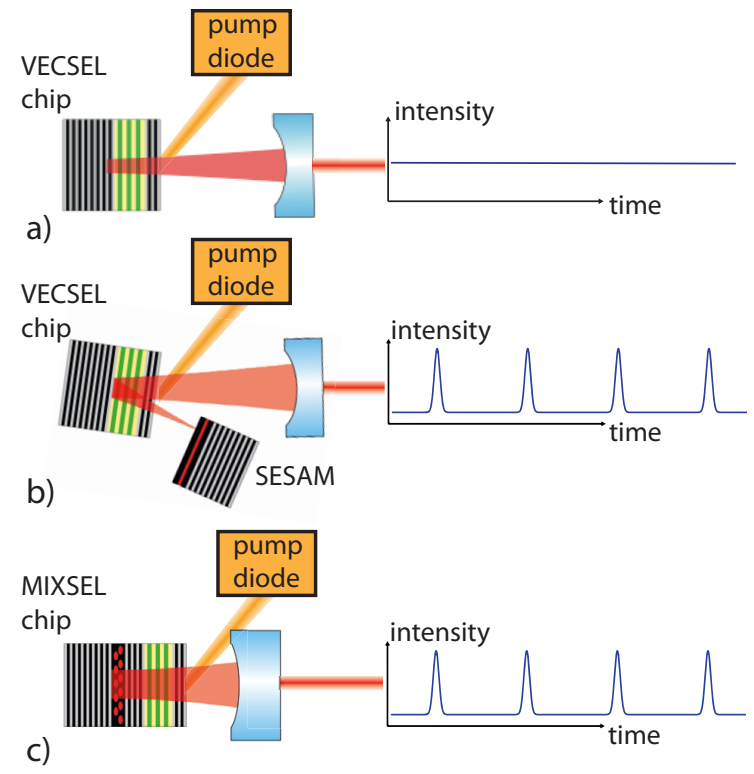

Figure 1.2: Semiconductor disk laser configurations: a) VECSEL chip and output coupling mirror form a straight linear cavity for continuous-wave operation; b) In a V-shaped cavity the VECSEL chip acts as a folding mirror while the SESAM and the output coupler form the end mirrors. This configuration is suited for modelocking operation. c) Since the MIXSEL chip combines the gain of a VECSEL chip and the saturable absorber of the SESAM, modelocking in a simple straight linear cavity is possible.

The VECSEL can emit cw light from a simple straight linear cavity in which the VECSEL chip and an output coupler form the two end mirrors (Fig. 1.2a). In single longitudinal and transversal mode up to $23 \mathrm{~W}$ of average output power has been achieved [17], and with multimode emission even up to $106 \mathrm{~W}$ [18]. To obtain pulsed operation, a semiconductor saturable absorber mirror (SESAM) can be inserted into the cavity (Fig. 1.2b). The saturable absorber of the SESAM leads to a nonlinear reflectivity with less losses in case of a high incident fluence compared to the case of a low fluence. Hence, it is energetically favorable for the laser to emit light in form of pulses instead of cw operation leading to passive modelocking. In 2000, the first SESAM-modelocked VECSEL produced pulses with a dura- 
tion of $22 \mathrm{ps}$ at an average output power of $22 \mathrm{~mW}$ and a peak power of $200 \mathrm{~mW}$ [19]. Since then, the performance has constantly been improved and peak power levels of up to $6.3 \mathrm{~kW}$ [20] and pulses as short as $96 \mathrm{fs}$ (see chapter 6) [21] have been obtained. In contrast to solid state lasers, SDLs do not suffer from Q-switching instabilities [22] due to the short upper-state lifetime and the high gain cross-section of the semiconductor material. Therefore, pulse repetition rates ranging from $100 \mathrm{MHz}$ [23] up to $100 \mathrm{GHz}$ [24] have been presented with fundamentally modelocked SDLs.

The V-shaped cavity of a SESAM-modelocked VECSEL is already very simple and compact. However, the compactness can still be increased. The VECSEL chip and the SESAM are based on the same semiconductor materials and include both a bottom distributed Bragg reflector (DBR). The integration of the saturable absorber of the SESAM into the gain structure of a VECSEL led to the modelocked integrated external-cavity surface emitting laser (MIXSEL), first demonstrated in 2007 [25]. The MIXSEL allows for fundamental modelocking in a simple straight linear cavity, only comprising the MIXSEL chip and an output coupler (Fig. 1.2c). Today, up to $6.4 \mathrm{~W}$ of average output power [26] and sub-200 fs pulses [27] have been presented with MIXSELs.

The main idea developed within the framework of this thesis is the concept of polarization duplexing for a modelocked cavity, which we also patented (International Patent, WO 2016/049787 A1 issued April 7, 2016) [28]. A birefringent crystal is inserted into a laser cavity to split the single cavity beam into two spatially separated and cross-polarized beams with slightly different optical path-lengths in the birefringent crystal. This enables the simultaneous emission of two modelocked beams from a single laser cavity. Applying this concept to the linear cavity of the MIXSEL led to the invention of the dual-comb MIXSEL. This thesis presents the first demonstration of the dual-comb MIXSEL and shows that such a simple, compact, and cost-efficient laser is capable of performing dual-comb spectroscopy measurements. This could drastically simplify dual-comb spectroscopy and lead to a widespread use and new applications of this technique. 
The thesis is structured in the following way: A key requirement for frequency metrology and spectroscopy is low noise operation of the laser. Chapter 2 contains the first detailed noise analysis of a MIXSEL including the stabilization of the pulse repetition rate. In chapter 3 the polarization duplexing method based on a birefringent crystal is explained and the dual-comb MIXSEL is presented. The interplay between the two beams in one cavity and their noise correlation is studied in detail in chapter 4 . The demonstration of a dual-comb SESAM-modelocked Nd:YAG laser shows that the concept can also be applied to other laser gain media such as diode-pumped solid-state lasers. With all requirements for dual-comb spectroscopy fulfilled, the first gas spectroscopy measurement of water vapor with the dual-comb MIXSEL is presented in chapter 5. Since both beams are emitted from the same cavity, dual-comb spectroscopy is even possible with a completely free-running dual-comb MIXSEL, i.e. without the stabilization of neither the pulse repetition frequencies nor the $\mathrm{CEO}$ frequencies. A microwave-only stabilization of the down-converted dualcomb enables also measurements with long integration times, for example in case of weak absorption. In order to increase the available optical bandwidth for future experiments, the pulse shortening strategies with the result of the first sub-100 fs SDL with an optical bandwidth of $17 \mathrm{~nm}$ full-width at half maximum (FWHM) are presented in chapter 6 . The knowledge about different noise characterization methods aquired during the work of this thesis is used to demonstrate that the pulse repetition rates and the CEO frequencies of the two counter-propagating beams in a colliding pulse modelocking (CPM) VECSEL are locked and that they can be coherently combined for power scaling in chapter 7. Finally, the work is concluded and an outlook on further improvements and directions of the dual-comb MIXSEL is given ins chapter 8.

The main results of chapters 2 to 7 are presented in journal publications. The text and figures are printed as published, only the format of the text, the numbering and the size of the figures were adapted to the style of this thesis. All papers are reprinted with permission. The copyright of the original publications are held by the respective copyright holders. 



\section{Noise analysis of a MIXSEL}

Low noise operation is a generally desired feature for laser oscillators, but it becomes especially important for applications in the field of optical metrology and optical frequency combs [6]. In this chapter, after a brief introduction of the theory of amplitude and timing phase noise from modelocked lasers in section 2.1, the noise of a MIXSEL in free-running as well as stabilized operation is analyzed in section 2.2. The results show that modelocked SDLs are well suited for applications relying on low-noise lasers.

\subsection{Noise characterization of a pulse train}

This section is modified from the comprehensive discussions in Ref. [29] with the notation adapted from Ref. [30].

\subsubsection{Ideal pulse train}

Assuming short pulse durations compared to the cavity roundtrip time $T_{\mathrm{r}}$, the time-dependent optical power $P(t)$ of an ideal noise-free pulse train emitted by a modelocked laser can be described by a sum of equally spaced Dirac delta functions

$$
P(t)=P_{\text {avg }} \cdot T_{\mathrm{r}} \cdot \sum_{\mathrm{n}=-\infty}^{+\infty} \delta\left(t-n \cdot T_{\mathrm{r}}\right),
$$


where $P_{\mathrm{av}}$ denotes the average output power. Applying the Wiener Khinchin theorem [29], we can derive the power spectral density (PSD)

$$
S_{\mathrm{P}}(f)=\left(P_{\text {avg }}\right)^{2} \cdot \sum_{\mathrm{n}=-\infty}^{+\infty} \delta\left(f-n \cdot f_{\text {rep }}\right),
$$

where $f_{\text {rep }}=1 / T_{\mathrm{r}}$ is the repetition frequency. This represents a comb of equidistantly spaced delta peaks at the frequencies $f_{\mathrm{n}}=n \cdot f_{\text {rep }}$.

\subsubsection{Amplitude noise}

A pulse train emitted from a real modelocked laser suffers from perturbations in form of timing phase noise and amplitude fluctuations. Taking the amplitude fluctuations (Fig 2.1a) into account, the time dependent optical power from equation (2.1) extends to

$$
P(t)=(1+N(t)) P_{\mathrm{avg}} \cdot T_{\mathrm{r}} \cdot \sum_{\mathrm{n}=-\infty}^{+\infty} \delta\left(t-n \cdot T_{\mathrm{r}}\right),
$$

where $N(t)$ describes the normalized power fluctuations. Under the assumption of an ergodic system [29] the Wiener-Khinchin theorem can be applied again leading to the according PSD

$$
S_{\mathrm{P}, \mathrm{A}}(f)=\left(P_{\text {avg }}\right)^{2} \cdot \sum_{\mathrm{n}=-\infty}^{+\infty}\left[\delta\left(f-n \cdot f_{\text {rep }}\right)+\left(\tilde{N}\left(f-n \cdot f_{\text {rep }}\right)\right)^{2}\right] .
$$

Here $\tilde{N}(f)$ represents the Fourier transformation of $N(t)$. The amplitude fluctuations (Fig 2.1a) add side bands to the delta peaks in the PSD (Fig 2.1b). These side bands are independent of the harmonic number $n$ assuming that coupling between amplitude and timing phase noise is neglected.

The integrated root mean square (rms) amplitude noise in the frequency range $\left[f_{\text {low }}, f_{\text {high }}\right]$ can be calculated from the PSD according to

$$
\left.\sigma_{\mathrm{A}}\right|_{\left[\mathrm{f}_{\mathrm{low}}, \mathrm{f}_{\text {high }}\right]}=\sqrt{2 \cdot \int_{\mathrm{f}_{\mathrm{low}}}^{f_{\text {high }}} S_{\mathrm{P}, \mathrm{A}}(f) d f .}
$$



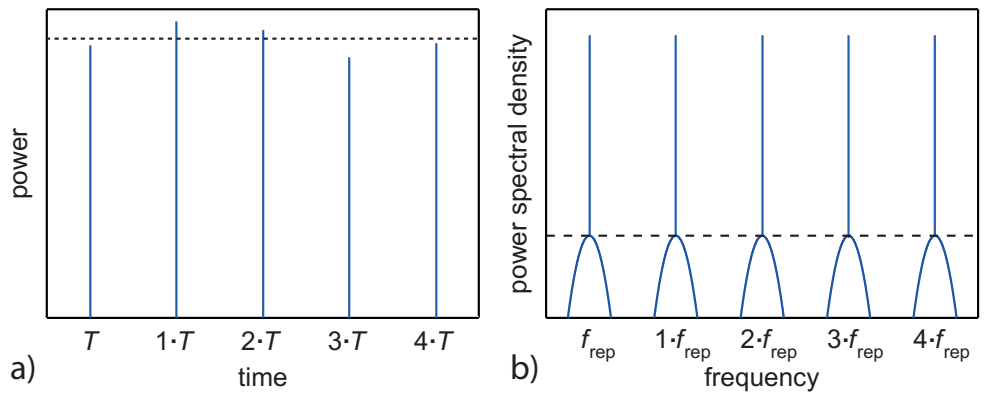

Figure 2.1: Pulse train with amplitude noise: a) power fluctuations in the time domain generate b) equal side bands for all the harmonics in the frequency domain.

The factor 2 in equation (2.5) is a result of the discrepancy between the definition of the PSD, which is two-sided, and the measured singlesideband PSD [31].

\subsubsection{Timing phase noise}

Fluctuations of the pulse roundtrip period $T_{r}$, referred to as timing phase noise, can be taken into account for the time dependent optical power by extending equation (2.1)

$$
P(t)=P_{\mathrm{avg}} \cdot T_{\mathrm{r}} \cdot \sum_{\mathrm{n}=-\infty}^{+\infty} \delta\left(t-n \cdot T_{\mathrm{r}}-\delta T_{\mathrm{r}}(t)\right) .
$$

The deviation from the ideal arrival time $t=n \cdot T_{\mathrm{r}}$ of the $n^{\text {th }}$ pulse (dotted lines in Fig 2.2a) is described by $\delta T_{\mathrm{r}}(t)$.

Using the shifting theorem of the Fourier transformation and the Wiener-Khinchin theorem and considering $\delta T_{\mathrm{r}}(t) / T_{\mathrm{r}} \ll 1$ one can calculate the PSD as:

$$
S_{\mathrm{P}, \mathrm{T}}(f)=\left(P_{\mathrm{avg}}\right)^{2} \cdot \sum_{\mathrm{n}=-\infty}^{+\infty}\left[\delta\left(f-n \cdot f_{\text {rep }}\right)+\left(n \cdot 2 \pi f_{\text {rep }} \cdot \delta \tilde{T}\left(f-n \cdot f_{\text {rep }}\right)\right)^{2}\right]
$$


where $\delta \tilde{T}(f)$ represents the Fourier transformation of $\delta T(t)$. The fluctuations of the pulse repetition rate add side bands to the peaks in the PSD (Fig 2.2b). In contrast to the amplitude noise, the side bands are not independent of the harmonic number $n$ but scale with $n^{2}$.
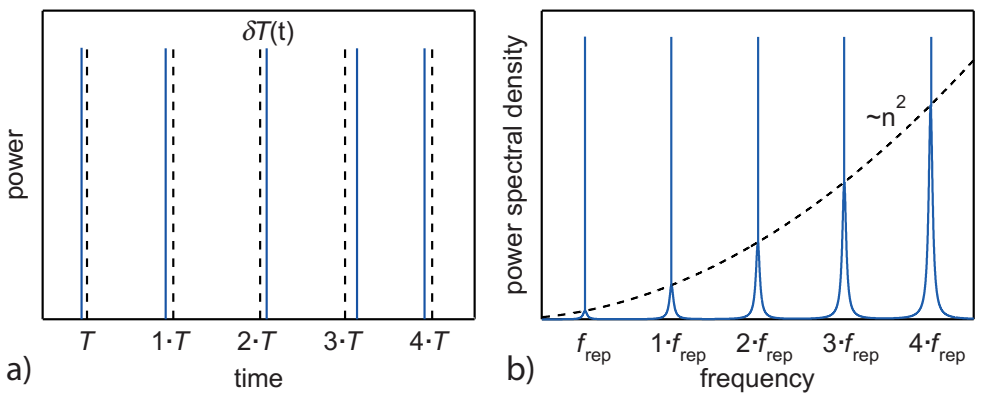

Figure 2.2: Pulse train with timing phase noise: a) fluctuations of the pulse arrival time with respect to the ideal case (dotted lines) result in b) side bands in the PSD that increase quadratically with the harmonic number $n$.

The integrated rms timing jitter $\sigma_{\mathrm{T}}$ can be used to quantify and compare the phase noise of different modelocked lasers over a frequency range $\left[f_{\text {low }}, f_{\text {high }}\right]$

$$
\left.\sigma_{\mathrm{T}}\right|_{\left[\mathrm{f}_{\text {low }}, \mathrm{f}_{\text {high }}\right]}=\frac{\sqrt{2 \cdot \int_{\mathrm{f}_{\text {low }}}^{f_{\text {high }}} S_{\mathrm{n}, \mathrm{P}, \mathrm{T}}(f) d f}}{2 \pi n f_{\text {rep }}} .
$$

The factor two arrises again due to the discrepancy between single- and two-sided PSD.

A detailed noise characterization of a MIXSEL with record noise performance is presented in the following IEEE Photonics Journal publication [32] (2014 IEEE. Reprinted, with permission, from M. Mangold, S. M. Link, A. Klenner, C. A. Zaugg, M. Golling, B. W. Tilma, U. Keller, Amplitude noise and timing jitter characterization of a high-power mode-locked integrated external-cavity surface emitting laser, IEEE Photonics Journal, Feb. 2014) 


\title{
2.2 Amplitude noise and timing jitter characterization of a high-power mode-locked integrated external-cavity surface emitting laser
}

\author{
M. Mangold, S. M. Link, A. Klenner, C. A. Zaugg, M. Golling, B. W.
}

Tilma, and U. Keller

Department of Physics, Institute for Quantum Electronics, ETH Zurich, 8093 Zurich, Switzerland

We present a timing jitter and amplitude noise characterization of a high-power mode-locked integrated external-cavity surface emitting laser (MIXSEL). In the MIXSEL, the semiconductor saturable absorber of a SESAM is integrated into the structure of a VECSEL to start and stabilize passive mode-locking. In comparison to previous noise characterization of SESAM-mode-locked VECSELs, this first noise characterization of a MIXSEL is performed at a much higher average output power. In a free-running operation, the laser generates 14.3-ps pulses at an average output power of $645 \mathrm{~mW}$ at a $2-\mathrm{GHz}$ pulse repetition rate and an RMS amplitude noise of $<0.15 \%$ [1 Hz, $10 \mathrm{MHz}]$. We measured an RMS timing jitter of $129 \mathrm{fs}$ [100 Hz, $10 \mathrm{MHz}$, which represents the lowest value for a free-running passively mode-locked semiconductor disk laser to date. Additionally, we stabilized the pulse repetition rate with a piezo actuator to control the cavity length. With the laser generating 16.7-ps pulses at an average output power of $701 \mathrm{~mW}$, the repetition frequency was phase-locked to a low-noise electronic reference using a feedback loop. In actively stabilized operation, the RMS timing jitter was reduced to less than $70 \mathrm{fs}[1 \mathrm{~Hz}, 100 \mathrm{MHz}$. In the $100-\mathrm{Hz}$ to $10-\mathrm{MHz}$ bandwidth, we report the lowest timing jitter measured from a passively mode-locked semiconductor disk laser to date with a value of $31 \mathrm{fs}$. These results show that the MIXSEL technology provides compact ultrafast laser sources combining high-power and low-noise performance similar to diode-pumped solid-state lasers, which enable world-record optical communication rates and low-noise frequency combs. 


\subsubsection{Introduction}

High power ultrafast laser sources with low timing jitter are required by numerous applications, for example in high-resolution optical sampling [1], frequency metrology [2], and high-class AD-converters [3]. In addition, modern ultra high-speed and high-bandwidth communication systems demand low-noise laser systems with gigahertz repetition rates [4] for which low-noise diode-pumped SESAM mode-locked solid-state lasers [5], [6] replaced fiber lasers. In comparison to the conventional bulk and fiber laser systems, the semiconductor disk laser technology can provide inexpensive wafer scale mass production at considerably lower costs, making this technology the most prominent candidate for widespread industrial applications [7].

Optically pumped vertical-external-cavity surface emitting lasers (VECSELs [8]), mode-locked with semiconductor saturable absorber mirrors (SESAMs [9], [10]), have recently shown remarkable progress with regard to high-power operation combined with femtosecond pulse durations [11][13]. The surface emitting disk geometry provides excellent beam quality, low intracavity nonlinearities and is power-scalable by simply increasing the laser mode area on the semiconductor disk [10]. Vertical integration of the saturable absorber of a SESAM led to the mode-locked integrated external-cavity surface emitting laser (MIXSEL [14]) combining the gain of VECSELs and the saturable absorber of a SESAM in a single semiconductor layer-stack. The absorber integration not only reduces the complexity of the laser system, but also reduces manufacturing costs and enables simpler device packaging. Stable and self-starting passive mode-locking can be achieved in highly compact straight cavities, which allow for straightforward repetition rate scaling by translating the output coupler in beamdirection.

After the first demonstration of a MIXSEL in 2007 with moderate output power [14], improvements of the quantum-dot (QD) saturable absorber and the thermal management resulted in a MIXSEL with a recordhigh average output power of $6.4 \mathrm{~W}$ in 28-ps pulses at a repetition rate of $2.5 \mathrm{GHz}$ [25]. Even at a repetition rate of $10 \mathrm{GHz}$ a very high average output power of $2.4 \mathrm{~W}$ in 17-ps pulses was obtained, which corresponds to the highest pulse energy of a mode-locked 10-GHz laser to date [15]. 
Most recently the first femtosecond MIXSEL has been demonstrated [16].

However, the noise performance of MIXSELs has not been characterized so far. In comparison to SESAM-mode-locked VECSELs [17]-[21] we would expect similar if not better performance. All semiconductor disk lasers (SDLs), such as VECSELs and MIXSELs, are typically operated with low-loss $(\approx 1-2 \%)$ cavities and repetition rates in the order of gigahertz (cavity roundtrip time of $<1 \mathrm{~ns}$ ). The photon lifetime in those cavities is typically in the order of $100 \mathrm{~ns}$ and therefore substantially longer than the semiconductor gain lifetime of a few nanoseconds (in comparison: the upper-state lifetime of commonly used rare-earth-doped gain media is in the order of a few micro-seconds to milliseconds). This configuration acts as a low-pass filter for spontaneous emission noise [22] and enables low amplitude noise operation. Furthermore, the much larger gain cross-section of the semiconductor material (in the order of $10^{-14} \mathrm{~cm}^{2}$ ) compared to diode-pumped solid-state lasers (typically in the order of $<10^{-19} \mathrm{~cm}^{2}$ ) enables stable mode-locking at high repetition rates without unwanted Q-switching instabilities [23]. These Q-switching instabilities become even worse for the small intracavity pulse energies in the gigahertz repetition rate regime [24]. Nevertheless, for a 10-GHz SESAM mode-locked diode-pumped solid-state laser a nearly quantum-noise limited timing jitter was achieved by a stable mechanical setup and moderate intracavity nonlinearities [6]. For SDLs one can expect similar noise performance, as the interaction of the pulse with the semiconductor gain region is limited to a length of $\approx 1-2$ micrometers compared to the propagation in the free-space cavity of several millimeters up to centimeters length. In addition, enhanced spontaneous emission noise arising from waveguide effects in mode-locked edge-emitting semiconductor lasers is not present in SDLs.

Different timing jitter measurements were performed for mode-locked VECSELs [17]-[21]. For a laser with a 2-GHz repetition rate, the lowest RMS timing jitter in free-running operation was $201 \mathrm{fs}$ over an integration bandwidth of [100 Hz, $100 \mathrm{MHz}$ [17]. Active stabilization of the cavity length using a piezo actuator reduced the RMS timing jitter to record-low $58 \mathrm{fs}$ [1 Hz, $100 \mathrm{MHz}$ [18]. For all results [17]-[21] the VECSEL gain chip was used as a cavity folding mirror which doubles the round-trip gain 
compared to a cavity configuration with the gain at the end of the linear cavity. In contrast, for the straight linear cavity of a MIXSEL the semiconductor structure forms one end mirror. Therefore, the laser setup can be reduced by at least one cavity component, which should give rise to an even better mechanical stability compared to a mode-locked VECSEL.

Here, we present a noise characterization of a high-power picosecond MIXSEL. In free-running operation at around a 2-GHz repetition rate, the laser generated 14.3-ps pulses with $645 \mathrm{~mW}$ of average output power with an excellent RMS amplitude noise of $<0.11 \%$ [ $1 \mathrm{~Hz}, 10 \mathrm{MHz}$. The average output power is therefore an order of magnitude higher than in all previous timing jitter measurements of SESAM mode-locked VECSELs [18]-[21]. We measured the single-sideband (SSB) timing phase noise power spectral density (PSD) with a commercial signal-source analyzer (SSA) and obtained an RMS timing jitter of $129 \mathrm{fs}$ [100 Hz, $10 \mathrm{MHz}$ ] and $60 \mathrm{fs}[300 \mathrm{~Hz}, 10 \mathrm{MHz}$. These are the lowest timing jitter values of a free-running passively mode-locked semiconductor disk laser to date. Furthermore, in a slightly altered laser configuration, the repetition rate was actively stabilized to an electronic reference oscillator using a piezo actuator that changes the cavity length. In this configuration the laser generates 16.7-ps pulses at $701 \mathrm{~mW}$ of average output power. In the actively stabilized operation we measured an RMS timing jitter of $31 \mathrm{fs}[100 \mathrm{~Hz}$, $100 \mathrm{MHz}$ ] which represents the lowest value from a passively mode-locked semiconductor disk laser in this frequency range. Thus, these measurements confirm the superior performance of MIXSELs compared to SESAM mode-locked VECSELs for reasons discussed above.

\subsubsection{Laser design and mode-locking performance}

The MIXSEL (Fig. 2.3(a)) combines the quantum-well (QW) gain of VECSELs with quantum-dot (QD) saturable absorbers of SESAMs in a single structure, where the saturable absorber starts and stabilizes fundamental passive mode-locking. A single layer of self-assembled InAs QDs is placed between an AlAs/GaAs distributed Bragg reflector (DBR) for the laser wavelength and an intermediate $\mathrm{AlAs} / \mathrm{Al}_{0.2} \mathrm{Ga}_{0.8} \mathrm{As} \mathrm{DBR}$ for the pump wavelength. The active region is based on seven $\operatorname{In}_{0.13} \mathrm{Ga}_{0.87} \mathrm{As}$ QWs, which are embedded in GaAs for pump light absorption. On top 
of the layer stack an anti-reflection section minimizes the losses for pump and laser light at the interface between air and the semiconductor structure. The MIXSEL structure was grown by molecular beam epitaxy and flip-chip bonded to a diamond grown by chemical vapor deposition. Detailed descriptions about the structure and the flip-chip bonding are given in Refs. [15], [25].
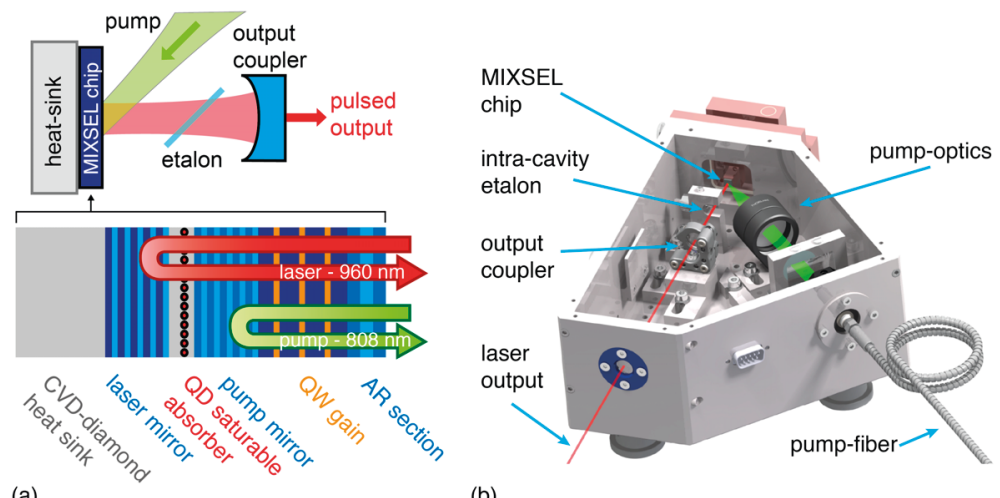

(a)

(b)

Figure 2.3: (a) MIXSEL structure. The single quantum-dot (QD) saturable absorber is placed between distributed Bragg reflectors for pump and lasing wavelength. The active region is based on InGaAs quantum-wells (QWs), followed by an antireflection (AR) section. The MIXSEL chip is inserted in a straight cavity with an intracavity AR-coated 20- $\mu \mathrm{m}$-thick fused silica etalon for wavelength tuning. The semiconductor chip is optically pumped under an angle of $45^{\circ}$. (b) MIXSEL housing to reduce external perturbation. The external 808-nm pump diode array is fibercoupled to the housing.

For optimum laser noise performance we reduced environmental influences with a stable metal housing designed and manufactured especially for this laser setup (Fig. 2.3(b)). In addition to improved mechanical and thermal stability, we reduced the unwanted airflow through the cavity with the closed housing. The MIXSEL chip is temperaturestabilized to minus $1{ }^{\circ} \mathrm{C}$ by a water-cooled Peltier element. Although water-cooling introduces additional vibrations of the housing, it is necessary to achieve high-power operation with any semiconductor disk laser. The laser is pumped at an angle of $45^{\circ}$ with up to $35 \mathrm{~W}$ by a water-cooled 
industrial-grade fiber-coupled 808-nm-pump-diode-array (LIMO35-F100DL808-LM) driven by a standard commercially available power supply (Delta Elektronika SM7.5-80). All pump optics are mounted tightly on the housing baseplate to guarantee the maximum stability and to avoid drifts caused by linear translation stages or adjustable lens mounts which are usually employed at university-based lasers. The flat MIXSEL chip and a curved output coupler (OC) form a straight linear cavity (Fig. 2.3(a), top). The cavity mode radius of $168 \mu \mathrm{m}$ on the gain chip was chosen to be slightly larger than the pump spot radius of $158 \mu \mathrm{m}$ to suppress higher order transverse mode operation. A $20-\mu \mathrm{m}$-thick anti-reflection-coated fused silica etalon is inserted in the cavity to tune and stabilize the lasing wavelength. The intracavity etalon bandwidth should support sub-1-ps pulses, but the long recovery time of the integrated quantum dot saturable absorber limits the pulse duration to $>10$ ps [26], [27].

In free-running operation we used an output coupler with 0.5 inch diameter, $200 \mathrm{~mm}$ radius of curvature (ROC) and a transmission of $0.3 \%$. At a pump power of $17.9 \mathrm{~W}$, stable mode-locking at a repetition rate of $1.985 \mathrm{GHz}$ was obtained, generating 14.3-ps pulses at an average output power of $645 \mathrm{~mW}$ and a center wavelength of $964.4 \mathrm{~nm}$ (Fig. 2.4).

For the active stabilization of the pulse repetition rate, as described
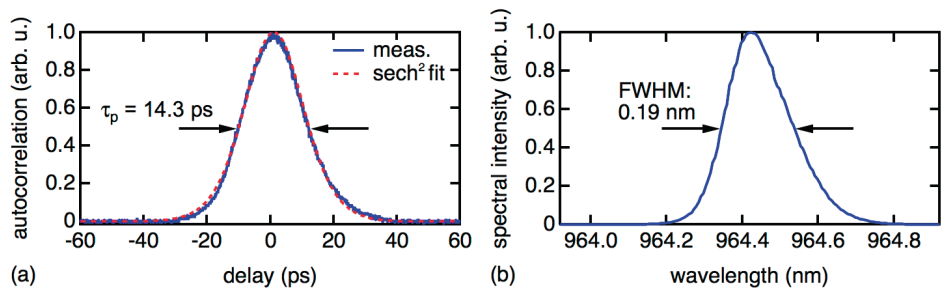

Figure 2.4: MIXSEL results in free-running operation at $645 \mathrm{~mW}$ average output power: (a) intensity autocorrelation with fit to an ideal sech $^{2}$-pulse of 14.3 ps duration, (b) optical spectrum centered at $964.4 \mathrm{~nm}$.

in Section 2.2.3, a different output coupler with a transmission of $0.5 \%$ and a ROC of $200 \mathrm{~mm}$ was used. To reduce the weight load on the piezo, an output coupler with a diameter of $2.5 \mathrm{~mm}$ and a thickness of $2 \mathrm{~mm}$ 


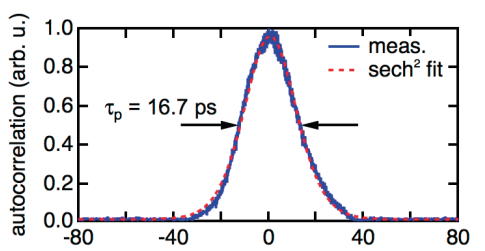

(a)

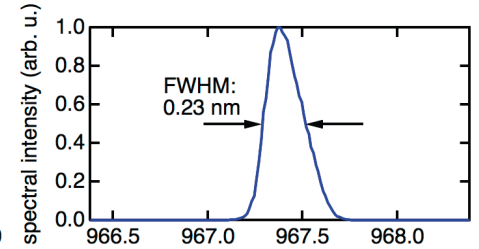

(b)

wavelength $(\mathrm{nm})$

Figure 2.5: MIXSEL results in stabilized operation at $701 \mathrm{~mW}$ average output power: (a) intensity autocorrelation with a fit to an ideal $\mathrm{sech}^{2}$-pulse of $16.7 \mathrm{ps}$ duration, (b) optical spectrum centered at $967.4 \mathrm{~nm}$.

was chosen (Fig 2.6(b)). At a heat-sink temperature of minus $1{ }^{\circ} \mathrm{C}$ and a pump power of $23 \mathrm{~W}$ stable mode-locking at a repetition rate of $2 \mathrm{GHz}$ was achieved (Fig. 2.4). In this configuration 16.7-ps pulses at an average output power of $701 \mathrm{~mW}$ were generated. In both configurations the laser was running stable over several hours of measurements without any signs of degradation.

\subsubsection{Timing phase noise characterization and stabilization setup}

For noise characterization in both, free-running and phase-locked operation, a fraction of the laser output power is detected by a highly linear photodiode (HLPD, Discovery Semiconductors Inc. DSC30S [28]). The detector signal is low-pass filtered at $2.4 \mathrm{GHz}$ to suppress harmonics of the repetition rate by $48 \mathrm{~dB}$. After low-noise amplification (Agilent $87405 \mathrm{C}$ ) of the signal to $+5 \mathrm{dBm}$, the SSB timing phase noise PSD was measured using a commercial SSA (Agilent E5052B) (Fig. 2.6(a), blue section). For an enhanced measurement sensitivity all timing phase noise measurements were performed with ten correlations [18].

To stabilize the pulse repetition rate of the laser, we used the piezocontrolled output coupler (OC) (Fig. 2.6(b)) to correct for any cavity length drifts. Therefore, an OC with a diameter of $2.5 \mathrm{~mm}$ was mounted on a piezoelectric ring actuator (maximum voltage: $200 \mathrm{~V}$, free-stroke: $2.7 \mu \mathrm{m}$ ) to pass the transmitted light (Fig. 2.6(b)). With the OC and its mounting 


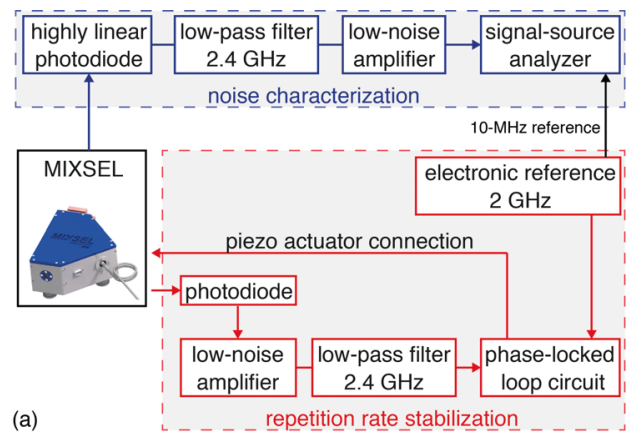

ring piezo actuator:

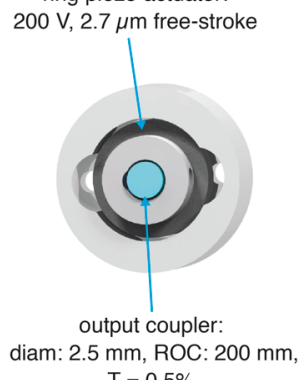

(b)

$\mathrm{T}=0.5 \%$

Figure 2.6: Schematic overview of the noise characterization and repetition-rate stabilization. The repetition-rate is stabilized to an electronic reference by a phaselocked loop (red section). For noise characterization (blue section) a part of the laser output is measured with a HLPD, low-pass filtered, amplified and the SSB timing phase noise PSD can be recorded with a commercial SSA. A stable common 10$\mathrm{MHz}$ reference signal from the reference oscillator is used as a frequency reference for the SSA. (b) Output coupler (diam.: $2.5 \mathrm{~mm}$, transmission: 0.5\%, ROC: $200 \mathrm{~mm}$ ) mounted on a ring piezo actuator $(200 \mathrm{~V}, 2.7 \mu \mathrm{m}$ free-stroke) for changing the length of the straight cavity.

plate weighing $\approx 230 \mathrm{mg}$, the $-3 \mathrm{~dB}$ operation bandwidth of the utilized actuator was measured to be around $1 \mathrm{kHz}$.

For the active stabilization, the repetition rate of the laser was measured with a 25-GHz InGaAs photodetector (NewFocus Model 1414). After amplification and a 2.4-GHz low-pass filter, the signal is used for stabilization with a custom-made phase-locked loop (PLL) [18]. The PLL is based on a double balanced mixer (DBM, MITEQ DM0104LA1) and a specifically designed Proportional-Integral (PI) loop filter. The DBM is used as an ana$\log$ phase detector and therefore seeded by the repetition frequency of the laser and a reference signal from a stable low noise electronic reference oscillator (Rohde\&Schwarz SMF100A). The phase error signal was filtered by the PI loop filter and sent to the piezo actuator. For the repetition rate stabilization over a time frame of about 1 hour a driving voltage of only $30 \mathrm{~V}$ was needed, which corresponds to a utilized stroke of $\approx 400 \mathrm{~nm}$. Note that the timing jitter is measured out-of-loop of the stabilization scheme. A stable common $10-\mathrm{MHz}$ reference signal from the electronic reference oscillator is used as a frequency reference for the SSA. 


\subsubsection{Measured timing phase noise results}

We measured the SSB timing phase noise PSD both in free-running and in actively stabilized operation with the measurement scheme presented in Section 2.2.3 (Fig. 2.7). For noise characterization of the laser in the different operation modes the photocurrent of the HLPD was chosen to be $0.6 \mathrm{~mA}$ in free-running and $1 \mathrm{~mA}$ in actively stabilized operation, which resulted in shot noise levels of $-144.5 \mathrm{dBc} / \mathrm{Hz}$ and $-154.9 \mathrm{dBc} / \mathrm{Hz}$, respectively. The photocurrents were chosen for a minimum shot noise level. However, in free-running operation excess amplitude to phase noise coupling in the detection set an upper limit to the photocurrent.

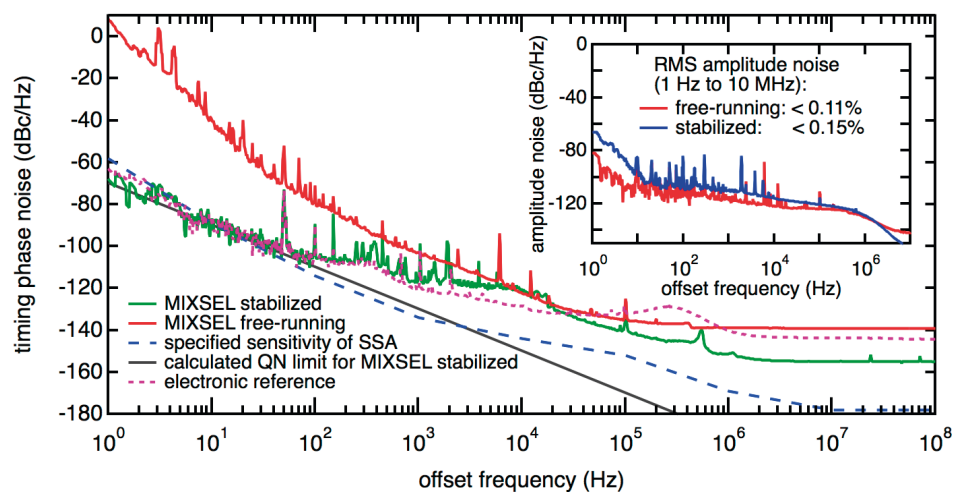

Figure 2.7: Noise characterization of a high-power MIXSEL: SSB timing phase noise PSD of free-running (solid red) and actively stabilized (solid green) laser. For comparison the PSD of the electronic reference (dashed purple), the specified sensitivity of the SSA (dashed blue) and the calculated QN limit (solid gray) are given. Inset: RMS amplitude noise of the free-running laser was $<0.11 \%[1 \mathrm{~Hz}, 10 \mathrm{MHz}]$ and of the stabilized laser was $<0.15 \%[1 \mathrm{~Hz}, 10 \mathrm{MHz}]$.

From the SSB timing phase noise PSD the RMS timing jitter in different frequency ranges between the lower limit $f_{\text {low }}$ and the higher limit $f_{\text {high }}$ is calculated. For all measurements we used the full bandwidth of the SSA of $\left(f_{\text {low }}=1 \mathrm{~Hz}\right.$ to $\left.f_{\text {high }}=100 \mathrm{MHz}\right)$, therefore timing jitter values for intermediate integration spans can be calculated. In free-running 
operation we measured an integrated RMS timing jitter of $129 \mathrm{fs}$ between $100 \mathrm{~Hz}$ and $10 \mathrm{MHz}$, which is the lowest value for free-running modelocked semiconductor disk laser in this integration range (Fig. 2.7, red solid line). Furthermore, an excellent amplitude noise of $<0.11 \%$ was obtained between $1 \mathrm{~Hz}$ and $10 \mathrm{MHz}$ in this configuration (Fig. 2.7 inset).

By phase-locking the repetition frequency to the low-noise electronic reference oscillator an integrated RMS timing jitter of 69 fs was measured for the integration between $1 \mathrm{~Hz}$ and $100 \mathrm{MHz}$ (Fig. 2.7, green solid line). The integrated RMS timing jitter between $100 \mathrm{~Hz}$ to $10 \mathrm{MHz}$ with a value of $31 \mathrm{fs}$ is lower than for any stabilized mode-locked semiconductor disk laser reported until now. For frequencies up to $1 \mathrm{kHz}$ the noise performance of the laser is limited by the electronic reference, which is in very good agreement with the measured actuator bandwidth. Between $1 \mathrm{kHz}$ and $10 \mathrm{kHz}$ the drop in actuator bandwidth causes deviations in the phase noise PSD from the electronic reference. The noise figure could be further improved with a piezo having a bandwidth greater than $10 \mathrm{kHz}$ even when loaded with the relatively heavy output coupler. Note that the noise performance of the stabilized laser is better than the timing phase noise of the electronic reference oscillator for frequencies above $20 \mathrm{kHz}$. In addition, for frequencies below $10 \mathrm{~Hz}$ the measured timing phase noise PSD is below the specified sensitivity of the SSA (Fig. 2.7, blue dashed line), which limits the measurement accuracy in this frequency range. For illustration the quantum noise (QN) limit (Fig. 2.7, grey solid line) was calculated for the stabilized laser configuration according to Ref. [29] with the laser parameters given in Section 2.2.2 for a sech'-pulse form and a saturated power small signal gain of 3\% [30]. The theoretical QN limited timing jitter is found to be proportional to the pulse duration and inversely proportional to the square-root of the pulse energy [31]. Therefore, shorter pulse durations for otherwise similar laser parameters should support even lower quantum noise limited timing jitter. A detailed overview of the noise performance in both operation modes is found in Table 2.1, together with timing jitter values of previously reported mode-locked VECSELs and a $10-\mathrm{GHz}$ diode-pumped solid-state laser. The integrated amplitude noise was $<0.15 \%[1 \mathrm{~Hz}, 10 \mathrm{MHz}]$ for the stabilized laser configuration (Fig. 2.7, inset). 
Table 2.1: Results of timing-jitter characterization of free-running and actively stabilized MIXSEL compared to different passively modelocked lasers with gigahertz pulse repetition rates [18]. All lasers are operated in fundamental mode-locking, i.e., with only one pulse per cavity roundtrip

\begin{tabular}{|c|c|c|c|c|c|}
\hline laser & $\begin{array}{l}\text { repetition } \\
\text { rate }(\mathrm{GHz})\end{array}$ & $\begin{array}{l}\text { output } \\
\text { power }(\mathrm{mW})\end{array}$ & $\begin{array}{l}f_{\text {low }} \\
(\mathrm{Hz})\end{array}$ & $\begin{array}{l}f_{\text {high }} \\
\text { (MHz) }\end{array}$ & $\begin{array}{l}\text { RMS timing } \\
\text { jitter (fs) }\end{array}$ \\
\hline \multicolumn{6}{|l|}{ MIXSEL } \\
\hline free-running & 1.985 & 645 & $\begin{array}{l}1 \\
100 \\
100 \\
300 \\
1000 \\
100\end{array}$ & $\begin{array}{l}100 \\
100 \\
1.56 \\
1.5 \\
15 \\
10\end{array}$ & $\begin{array}{l}141721 \\
145 \\
128 \\
56 \\
43.1 \\
129\end{array}$ \\
\hline stabilized & 2.004 & 701 & $\begin{array}{l}1 \\
100 \\
6 \\
300 \\
1000 \\
100\end{array}$ & $\begin{array}{l}100 \\
100 \\
1.56 \\
1.5 \\
15 \\
10\end{array}$ & $\begin{array}{l}69 \\
32 \\
45 \\
24 \\
25 \\
31\end{array}$ \\
\hline \multicolumn{6}{|l|}{ VECSEL } \\
\hline free-running [17] & 2 & 40 & $\begin{array}{l}100 \\
100\end{array}$ & $\begin{array}{l}10 \\
100\end{array}$ & $\begin{array}{l}200 \\
201\end{array}$ \\
\hline stabilized [18] & 2 & 40 & $\begin{array}{l}1 \\
100\end{array}$ & $\begin{array}{l}100 \\
100\end{array}$ & $\begin{array}{l}58 \\
47\end{array}$ \\
\hline free-running [20] & 0.897 & 40 & 1000 & 15 & 410 \\
\hline stabilized [20] & 0.897 & 40 & 1000 & 15 & 160 \\
\hline stabilized [19] & 1 & 40 & 300 & 1.5 & 190 \\
\hline free-running [21] & 1.68 & 21 & 100 & 10 & 8000 \\
\hline stabilized [21] & 1.68 & 21 & 100 & 10 & 423 \\
\hline \multicolumn{6}{|l|}{ Er:Yb:glass laser } \\
\hline free-running [6] & 10 & 15 & 100 & 1.56 & 190 \\
\hline stabilized [6] & 10 & 15 & 6 & 1.56 & 26 \\
\hline
\end{tabular}




\subsubsection{Conclusion and outlook}

We presented amplitude noise and timing jitter measurements of a freerunning and an actively stabilized high-power MIXSEL at a repetition rate of $2 \mathrm{GHz}$. Record-low noise performance was achieved with and without stabilization, even though we used a water-cooled MIXSEL chip mount that can introduce mechanical vibrations. In free-running operation the laser generated 14.3-ps pulses at an average output power of $645 \mathrm{~mW}$. By measuring the SSB timing phase noise PSD with an SSA, we obtained RMS timing jitters of $129 \mathrm{fs}$ [100 Hz, $10 \mathrm{MHz}]$ and $60 \mathrm{fs}[300 \mathrm{~Hz}, 10 \mathrm{MHz}$, which have been the lowest values for a free-running mode-locked semiconductor disk laser to date. In a slightly different laser configuration generating $701 \mathrm{~mW}$ of average output power in 16.7-ps pulses the repetition rate was stabilized. Phase-locking the repetition frequency to a lownoise electronic reference using a standard analog PLL greatly reduced the phase noise and resulted in an RMS timing-jitter of less than $70 \mathrm{fs}[1 \mathrm{~Hz}$, $100 \mathrm{MHz}$ ]. In the range between $100 \mathrm{~Hz}$ and $10 \mathrm{MHz}$ we report the lowest timing jitter from a passively mode-locked semiconductor disk laser with a value of $31 \mathrm{fs}$. These record-low timing phase noise properties are also supported by an excellent amplitude noise of $<0.15 \%$ [1 Hz, $10 \mathrm{MHz}$ ] for both the free-running and phase-locked operation with an average output power more than an order of magnitude higher than in all previous timing jitter measurements of SESAM-mode-locked VECSELs. Therefore, the excellent noise performance combined with high-power operation makes the MIXSEL a unique ultrafast laser source with adjustable pulse repetition rates in the gigahertz regime and high pulse energies [15]. Such a laser is ideally suited for applications in ultra high-speed optical clocking or high-resolution optical sampling.

Acknowledgement The authors acknowledge support of the technology and cleanroom facility FIRST of ETH Zurich for advanced micro- and nanotechnology.

\subsubsection{References}

[1] P. W. Juodawlkis, J. C. Twichell, G. E. Betts, J. J. Hargreaves, R. D. Younger, J. L. Wasserman, F. J. O'Donnell, K. G. Ray, and R. C. 
Williamson, "Optically sampled analog-to-digital converters," IEEE Trans. Microw. Theory Tech., vol. 49, no. 10, pp. 1840-1853, Oct. 2001.

[2] H. R. Telle, G. Steinmeyer, A. E. Dunlop, J. Stenger, D. H. Sutter, and U. Keller, "Carrier-envelope offset phase control: A novel concept for absolute optical frequency measurement and ultrashort pulse generation," Appl. Phys. B, vol. 69, no. 4, pp. 327-332, Oct. 1999.

[3] S. Rausch, T. Binhammer, A. Harth, J. Kim, R. Ell, F. X. Kärtner, and U. Morgner, "Controlled waveforms on the single-cycle scale from a femtosecond oscillator," Opt. Exp., vol. 16, no. 13, pp. 9739-9745, Jun. 2008.

[4] D. Hillerkuss, R. Schmogrow, T. Schellinger, M. Jordan, M. Winter, G. Huber, T. Vallaitis, R. Bonk, P. Kleinow, F. Frey, M. Roeger, S. Koenig, A. Ludwig, A. Marculescu, J. Li, M. Hoh, M. Dreschmann, J. Meyer, S. Ben Ezra, N. Narkiss, B. Nebendahl, F. Parmigiani, P. Petropoulos, B. Resan, A. Oehler, K. Weingarten, T. Ellermeyer, J. Lutz, M. Moeller, M. Huebner, J. Becker, C. Koos, W. Freude, and J. Leuthold, " $26 \mathrm{Tbit} \mathrm{s}^{-1}$ line-rate super-channel transmission utilizing all-optical fast Fourier transform processing," Nat. Photon., vol. 5, no. 6, pp. 364-371, Jun. 2011.

[5] A. E. H. Oehler, M. C. Stumpf, S. Pekarek, T. Südmeyer, K. J. Weingarten, and U. Keller, "Picosecond diode-pumped $1.5 \mu \mathrm{m} \mathrm{Er,} \mathrm{Yb:}$ Glass lasers operating at 10-100 GHz repetition rate," Appl. Phys. B, vol. 99, no. 1/2, pp. 53-62, Apr. 2010.

[6] A. Schlatter, B. Rudin, S. C. Zeller, R. Paschotta, G. J. Spühler, L. Krainer, N. Haverkamp, H. R. Telle, and U. Keller, “Nearly quantumnoise-limited timing jitter from miniature Er: Yb: Glass lasers," Opt. Lett., vol. 30, no. 12, pp. 1536-1538, Jun. 15, 2005.

[7] A.-R. Bellancourt, D. J. H. C. Maas, B. Rudin, M. Golling, T. Südmeyer, and U. Keller, "Modelocked Integrated External-Cavity Surface Emitting Laser (MIXSEL)," IET Optoelectron., vol. 3, no. 2, pp. 61-72, Apr. 2009.

[8] M. Kuznetsov, F. Hakimi, R. Sprague, and A. Mooradian, "High- 
power ( $>0.5-\mathrm{W} \mathrm{CW}$ ) diode-pumped vertical-external-cavity surfaceemitting semiconductor lasers with circular TEM00 beams," IEEE Photon. Technol. Lett., vol. 9, no. 8, pp. 1063-65, Aug. 1997.

[9] U. Keller, K. J. Weingarten, F. X. Kärtner, D. Kopf, B. Braun, I. D. Jung, R. Fluck, C. Hönninger, N. Matuschek, and J. Aus der Au, "Semiconductor Saturable Absorber Mirrors (SESAMs) for femtosecond to nanosecond pulse generation in solid-state lasers," IEEE J. Sel. Topics Quantum Electron., vol. 2, no. 3, pp. 435-453, Sep. 1996.

[10] U. Keller and A. C. Tropper, "Passively modelocked surface-emitting semiconductor lasers," Phys. Rep., vol. 429, no. 2, pp. 67-120, Jun. 2006.

[11] M. Scheller, T. L. Wang, B. Kunert, W. Stolz, S. W. Koch, and J. V. Moloney, "Passively modelocked VECSEL emitting 682 fs pulses with $5.1 \mathrm{~W}$ of average output power," Electron. Lett., vol. 48, no. 10, pp. 588-589, May 2012.

[12] K. G. Wilcox, A. C. Tropper, H. E. Beere, D. A. Ritchie, B. Kunert, B. Heinen, and W. Stolz, " $4.35 \mathrm{~kW}$ peak power femtosecond pulse mode-locked VECSEL for supercontinuum generation," Opt. Exp., vol. 21, no. 2, pp. 1599-1605, Jan. 2013.

[13] M. Hoffmann, O. D. Sieber, V. J. Wittwer, I. L. Krestnikov, D. A. Livshits, Y. Barbarin, T. Südmeyer, and U. Keller, "Femtosecond highpower quantum dot vertical external cavity surface emitting laser," Opt. Exp., vol. 19, no. 9, pp. 8108-8116, Apr. 2011.

[14] D. J. H. C. Maas, A.-R. Bellancourt, B. Rudin, M. Golling, H. J. Unold, T. Südmeyer, and U. Keller, "Vertical integration of ultrafast semiconductor lasers," Appl. Phys. B, vol. 88, no. 4, pp. 493-497, Sep. 2007.

[15] V. J. Wittwer, M. Mangold, M. Hoffmann, O. D. Sieber, M. Golling, T. Südmeyer, and U. Keller, "High-power integrated ultrafast semiconductor disk laser: Multi-Watt $10 \mathrm{GHz}$ pulse generation," Electron. Lett., vol. 48, no. 18, pp. 1144-1145, Aug. 2012.

[16] M. Mangold, V. J. Wittwer, C. A. Zaugg, S. M. Link, M. Golling, B. W. Tilma, and U. Keller, "Femtosecond pulses from a Modelocked 
Integrated External-Cavity Surface Emitting Laser (MIXSEL)," Opt. Exp., vol. 21, no. 21, pp. 24 904-24 911, Oct. 2013.

[17] V. J. Wittwer, C. A. Zaugg, W. P. Pallmann, A. E. H. Oehler, B. Rudin, M. Hoffmann, M. Golling, Y. Barbarin, T. Südmeyer, and U. Keller, "Timing jitter characterization of a free-running SESAM mode-locked VECSEL," IEEE Photon. J., vol. 3, no. 4, pp. 658-664, Aug. 2011.

[18] V. J. Wittwer, R. van der Linden, B. W. Tilma, B. Resan, K. J. Weingarten, T. Südmeyer, and U. Keller, "Sub-60-fs timing jitter of a SESAM modelocked VECSEL," IEEE Photon. J., vol. 5, no. 1, p. 1400107, Feb. 2013.

[19] A. H. Quarterman, K. G. Wilcox, S. P. Elsmere, Z. Mihoubi, and A. C. Tropper, "Active stabilisation and timing jitter characterisation of sub-500 fs pulse passively modelocked VECSEL," Electron. Lett., vol. 44, no. 19, pp. 1135-1137, Sep. 2008.

[20] K. G. Wilcox, H. D. Foreman, J. S. Roberts, and A. C. Tropper, “Timing jitter of $897 \mathrm{MHz}$ optical pulse train from actively stabilised passively modelocked surface-emitting semiconductor laser," Electron. Lett., vol. 42, no. 3, pp. 159-160, Feb. 2006.

[21] G. Baili, M. Alouini, L. Morvan, D. Dolfi, A. Khadour, S. Bouchoule, and J. L. Oudar, "Timing jitter reduction of a mode-locked VECSEL using an optically triggered SESAM," IEEE Photon. Technol. Lett., vol. 22, no. 19, pp. 1434-1436, Oct. 2010.

[22] M. Myara, M. Sellahi, A. Laurain, A. Michon, I. Sagnes, and A. Garnache, "Noise properties of NIR and MIR VECSELs," in Proc. SPIE Vertical External Cavity Surf. Emitting Lasers III, 2013, pp. 86060Q1-86060Q-13.

[23] C. Hönninger, R. Paschotta, F. Morier-Genoud, M. Moser, and U. Keller, "Q-switching stability limits of continuous-wave passive mode locking," J. Opt. Soc. Amer. B, Opt. Phys., vol. 16, no. 1, pp. 46-56, Jan. 1999.

[24] ] R. Paschotta, L. Krainer, S. Lecomte, G. J. Spühler, S. C. Zeller, A. Aschwanden, D. Lorenser, H. J. Unold, K. J. Weingarten, and U. 
Keller, "Picosecond pulse sources with multi-GHz repetition rates and high output power," New J. Phys., vol. 6, no. 1, p. 174, Nov. 2004.

[25] B. Rudin, V. J. Wittwer, D. J. H. C. Maas, M. Hoffmann, O. D. Sieber, Y. Barbarin, M. Golling, T. Südmeyer, and U. Keller, "High-power MIXSEL: An integrated ultrafast semiconductor laser with $6.4 \mathrm{~W}$ average power," Opt. Exp., vol. 18, no. 26, pp. 27 582-27 588, Dec. 2010.

[26] O. Sieber, M. Hoffmann, V. Wittwer, M. Mangold, M. Golling, B. Tilma, T. Südmeyer, and U. Keller, "Experimentally verified pulse formation model for high-power femtosecond VECSELs," Appl. Phys. B, vol. 113, no. 1, pp. 133-145, Oct. 2013.

[27] D. J. H. C. Maas, A. R. Bellancourt, M. Hoffmann, B. Rudin, Y. Barbarin, M. Golling, T. Südmeyer, and U. Keller, "Growth parameter optimization for fast quantum dot SESAMs," Opt. Exp., vol. 16, no. 23, pp. 18 646-18 656, Nov. 2008.

[28] A. Joshi and S. Datta, "Dual InGaAs photodiodes having high phase linearity for precise timing applications," IEEE Photon. Technol. Lett., vol. 21, no. 19, pp. 1360-1362, Oct. 2009.

[29] R. Paschotta, "Noise of mode-locked lasers. Part II: Timing jitter and other fluctuations," Appl. Phys. B, vol. 79, no. 2, pp. 163-173, Jul. 2004.

[30] M. Mangold, V. J. Wittwer, O. D. Sieber, M. Hoffmann, I. L. Krestnikov, D. A. Livshits, M. Golling, T. Südmeyer, and U. Keller, “VECSEL gain characterization," Opt. Exp., vol. 20, no. 4, pp. 4136-4148, Feb. 2012.

[31] R. Paschotta, H. R. Telle, and U. Keller, "Noise of solid-state lasers," in Solid-State Lasers and Applications, A. Sennaroglu, Ed. Boca Raton, FL, USA: CRC Press, 2007, pp. 473-510. 


\section{Dual-comb MIXSEL}

The excellent noise performance of SDLs and especially the MIXSEL allow the use of these lasers for sensitive applications in the field of optical metrology. Due to the rapid development and progress of optical frequency combs over the last years, many new and exciting applications based on this technology emerged. In particular the use of two optical frequency combs with a slightly different comb spacing has enabled new applications such as dual-comb spectroscopy [5] and asynchronous optical sampling (ASOPS) [33] which are tremendously decreasing the acquisition time and increasing the accuracy compared to previous measurement techniques. However, currently the complexity and the high costs of having two optical frequency combs that are very stable in respect to each other limits these applications from a widespread use outside of the research laboratory. In this chapter, a new method is presented that enables the simultaneous generation of two optical frequency combs from a single semiconductor disk laser, drastically simplifying the dual-comb approach. In section 3.1 the principal idea of polarization duplexing using a birefringent crystal is introduced and in section 3.2 the method is applied to demonstrate the first dual-comb MIXSEL.

\subsection{Polarization duplexing}

The idea of the polarization duplexing method is to split an unpolarized beam into two cross-polarized and spatially separated beams using a bire- 
fringent crystal inside the laser cavity (Fig 3.1a). The polarization orthogonal (indicated by the circles in Fig 3.1a) to the optical axis of the birefringent crystal passes through the crystal in a straight line. However, the polarization turned by $90^{\circ}$ (indicated by two-sided arrows in Fig 3.1a) compared to the orthogonal one is deflected under a walk-off angle $\rho$. The reason for this is that a birefringent material has two different refractive indices, $n_{\mathrm{o}}$ and $n_{\mathrm{e}}$, for the ordinary and the extraordinary axis, respectively [34]. The energy will flow along the pointing vector $\vec{S}$ under this angle $\rho$ for the second polarization. The $\vec{k}$ vector, however, is still parallel to the $\vec{k}$ vector of the other polarization. Therefore, both beams will exit the birefringent crystal spatially separated but parallel, assuming normal incidence on the crystal. The distance $d$ between the two beams can be calculated with the length of the crystal $L$ and $\rho$

$$
d=L \cdot \tan (\rho) .
$$

The walk-off angle $\rho$ depends on the refractive indices $n_{\mathrm{o}}$ and $n_{\mathrm{e}}$ and the angle $\Psi$

$$
\tan (\rho)=\frac{\left|n_{\mathrm{e}}^{2}-n_{\mathrm{o}}^{2}\right| \sin (\Psi) \cos (\Psi)}{\left(n_{\mathrm{o}} \cos (\Psi)\right)^{2}+\left(n_{\mathrm{e}} \sin (\Psi)\right)^{2}}
$$

where $\Psi$ is the angle between the electric displacement field vector $\vec{D}$ and the optical axis of the birefringent crystal (Fig 3.1a).

Calcium carbonate $\left(\mathrm{CaCO}_{3}\right)$ is a convenient birefringent material to use inside a laser cavity since it combines a relatively large difference for the refractive indices ( $n_{\mathrm{o}}=1.645$ and $n_{\mathrm{e}}=1.480$ at a wavelength of $968 \mathrm{~nm}$ ) with very low group delay dispersion (GDD) values of $52 \mathrm{fs}^{2} / \mathrm{mm}$ and $33 \mathrm{fs}^{2} / \mathrm{mm}$ for the ordinary and the extraordinary axis, respectively. If $d$ should be maximized for normal incidence to get the biggest possible spatial separation of the two beams, the angle of the optical axis of the birefringent crystal has to be chosen accordingly. Fig 3.1b shows that $\tan (\rho)$ is maximal for an angle $\Psi$ of $48^{\circ}$ (black dotted line) with a value of 0.106 (for $\mathrm{CaCO}_{3}$, using Eq. 3.2). For all following experiments we used $\left(\mathrm{CaCO}_{3}\right)$ crystals with the optical axis under an angle of $45^{\circ}$ (red dotted line in Fig 3.1b) since the value for $\tan (\rho)$ at this angle is with 0.105 very close to the maximum value and a $45^{\circ}$ optical axis is more easily 

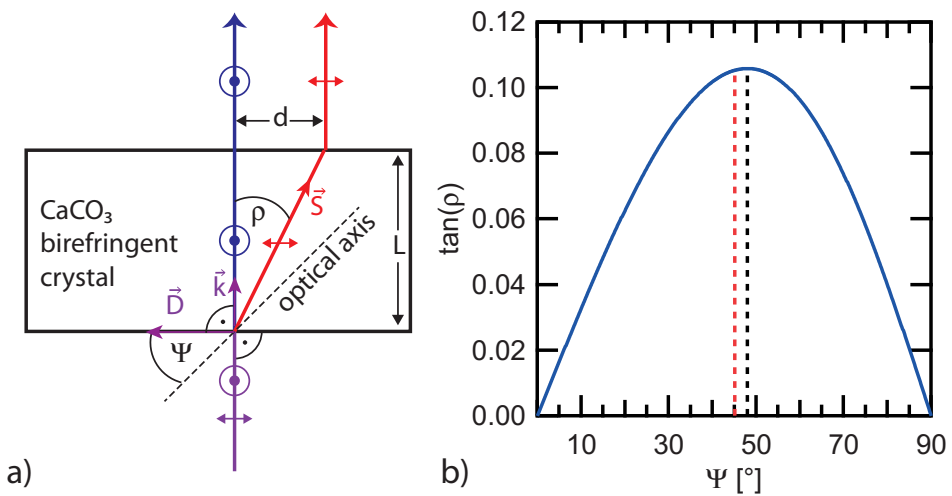

Figure 3.1: Beam propagation in birefringent crystal: a) an unpolarized beam is split into two spatially separated and cross-polarized beams. The polarization orthogonal to the optical axis of the birefringent crystal continues straight through the crystal. The polarization rotated by $90^{\circ}$ compared to the first polarization is deflected under a walk-off angle $\rho$ and continues parallel to the first polarization behind the crystal. b) The walk-off angle $\rho$ depends on angle $\Psi$ between the optical axis of the crystal and the electric displacement field vector $\vec{D}$ of the beam. The values for the birefringent crystal are taken for $\mathrm{CaCO}_{3}$ at a wavelength of $968 \mathrm{~nm}$.

commercially available. From equation 3.2 it follows that an unpolarized beam entering a 2-mm long $\mathrm{CaCO}_{3}$ crystal under normal incidence will be split into two cross-polarized beams with a spatial separation of $210 \mu \mathrm{m}$ after the crystal.

The MIXSEL is the ideal modelocked laser to apply the polarization duplexing method, since its simple straight linear cavity drastically facilitates the implementation. The first demonstration of a dual-comb MIXSEL is presented in the following Optics Express publication [35]. (C) 2015 Optical Society of America. One print or electronic copy may be made for personal use only. Systematic reproduction and distribution, duplication of any material in this paper for a fee or for commercial purposes, or modifications of the content of this paper are prohibited.) 


\subsection{Dual-comb modelocked laser}

Sandro M. Link, Alexander Klenner, Mario Mangold, Christian A. Zaugg, Matthias Golling, Bauke W. Tilma and Ursula Keller

Department of Physics, Institute for Quantum Electronics, ETH Zürich, 8093 Zürich, Switzerland.

In this paper we present the first semiconductor disk laser (SDL) emitting simultaneously two collinearly overlapping cross-polarized gigahertz modelocked pulse trains with different pulse repetition rates. Using only a simple photo detector and a microwave spectrum analyzer directly down-converts the frequency comb difference from the optical to the microwave frequency domain. With this setup, the relative carrier-envelope-offset (CEO) frequency can be accessed directly without an f-to-2f interferometer. A very compact design is obtained using the modelocked integrated external-cavity surface emitting laser (MIXSEL) which is part of the family of optically pumped SDLs and similar to a vertical external cavity surface emitting laser (VECSEL) but with both gain and saturable absorber integrated into the same semiconductor wafer (i.e. MIXSEL chip). We then simply added an additional intracavity birefringent crystal inside the linear straight cavity between the output coupler and the MIXSEL chip which splits the cavity beam into two collinear but spatially separated cross-polarized beams on the MIXSEL chip. This results in two modelocked collinear and fully overlapping cross-polarized output beams with adjustable pulse repetition frequencies with excellent noise performance. We stabilized both pulse repetition rates of the dual comb MIXSEL.

\subsubsection{Introduction}

Numerous frequency comb applications are based on two modelocked lasers with slightly different pulse repetition rates. One specific application is dual-comb spectroscopy [1-5]. Using heterodyne detection with two frequency combs offers excellent accuracy and very high sampling rates because no mechanical scanning technique is required. Another application benefiting from two modelocked lasers is asynchronous optical 
sampling (ASOPS) [6] which allows for pump-probe measurements without mechanical delay scanners. However, two separate modelocked lasers is complex, bulky, expensive and needs special stabilization efforts. Therefore, these applications would greatly benefit from a simple, compact and cost-efficient way of generating two modelocked pulse trains. Here we present a very simple solution which becomes even more attractive and more compact with optically pumped semiconductor disk lasers (SDLs).

Optically pumped SDLs is a family of vertical emitting semiconductor lasers with the first device demonstration referred to as the vertical external-cavity surface emitting laser (VECSEL) [7]. They have become very attractive because of their large spectral range of operation ranging from the visible to the infrared [8-10] and with intracavity frequency conversion covering high power operation from the visible to the UV [11, 12]. Modelocking was achieved with a semiconductor saturable absorber mirror (SESAM) $[13,14]$ the first time in $2000[15,16]$. Since then, SESAMmodelocked VECSELs generated pulses as short as $107 \mathrm{fs}$ [17] and peak power levels of up to $4.35 \mathrm{~kW}$ [18]. The first detection of the modelocked frequency comb offset, i.e. the carrier-envelope offset (CEO) frequency [19], from an amplified and pulse-compressed SESAM-modelocked VECSEL [20] has demonstrated the potential for low-noise frequency comb generation using optically pumped SDLs. As VECSEL and SESAM in most cases rely on the same semiconductor material system and fabrication techniques, the vertical integration of the saturable absorber into the gain structure of a VECSEL was the next level of integration to reduce complexity of ultrafast SDLs. Hence in 2007 the first modelocked integrated external-cavity surface emitting laser (MIXSEL) has been demonstrated [21]. In contrast to a MIXSEL, a SESAM-modelocked VECSEL has two separate semiconductor elements in the modelocked laser resonator, i.e. the VECSEL chip and the SESAM. With a MIXSEL a simple straight cavity is formed comprising the gain chip and an output coupler. The total cavity length determined by the MIXSEL chip (which adds less than $10 \mu \mathrm{m}$ to the cavity length) and the air space before the output coupler then sets the pulse repetition rate. This technology allows for stable and self-starting fundamental modelocking. An average output power of up to $6.4 \mathrm{~W}$ [22] and also femtosecond operation [23] has been demonstrated 
with a MIXSEL. Using the same MIXSEL chip and changing the cavity length we recently could adjust the pulse repetition rate between 5 and $100 \mathrm{GHz}$ [24]. In addition, MIXSELs have demonstrated very low noise performance similar to diode-pumped solid-state lasers [25].

In this paper we demonstrate for the first time a dual comb MIXSEL. With the MIXSEL device we substantially reduce complexity for dual comb generation because of the straight linear cavity made possible by the additional integration of the gain and absorber layers inside the same semiconductor chip. We then applied a method that has already been used in continuous wave $(\mathrm{cw})$ operation to generate dual frequency emission from an optically pumped VECSEL [26, 27]. We insert a birefringent crystal into the cavity to separate the modelocked cavity beam into two spatially separated beams with perpendicular polarization on the MIXSEL chip. This results in the first SDL emitting simultaneously two different gigahertz modelocked pulse trains from a single gain chip using the same cavity components. The microwave frequency comb-lines are the beat signal between the optical frequencies of the two modelocked laser beams observed with a photo detector and a microwave spectrum analyzer (MSA) even without any active cavity length stabilization. In addition with a second birefringent crystal placed inside the cavity to compensate the different path length introduced with the first crystal we can obtain two pulse trains with the same pulse repetition frequencies. This gives direct access to the relative $\mathrm{CEO}$ frequency between the two pulse trains without using an f-to-2f interferometer [19]. In addition, we show that both pulse repetition frequencies can be stabilized.

Recently, dual frequency comb spectroscopy was presented using only one Kerr-lens modelocked Yb:YAG thin disk oscillator, by creating a second beam with a slightly lower repetition frequency using an acoustooptic programmable dispersive filter (AOPDF) [28]. The two beams were directed through a gas cell to measure absorption features of acetylene. The dual-comb MIXSEL approach presented in this manuscript could be potentially used for similar gas spectroscopy, with the benefit of providing both lasers directly from a compact laser cavity. The absorption of one or several optical lines of the lasers in the gas cell can be directly detected in a decrease in amplitude of the corresponding beat-lines of the 


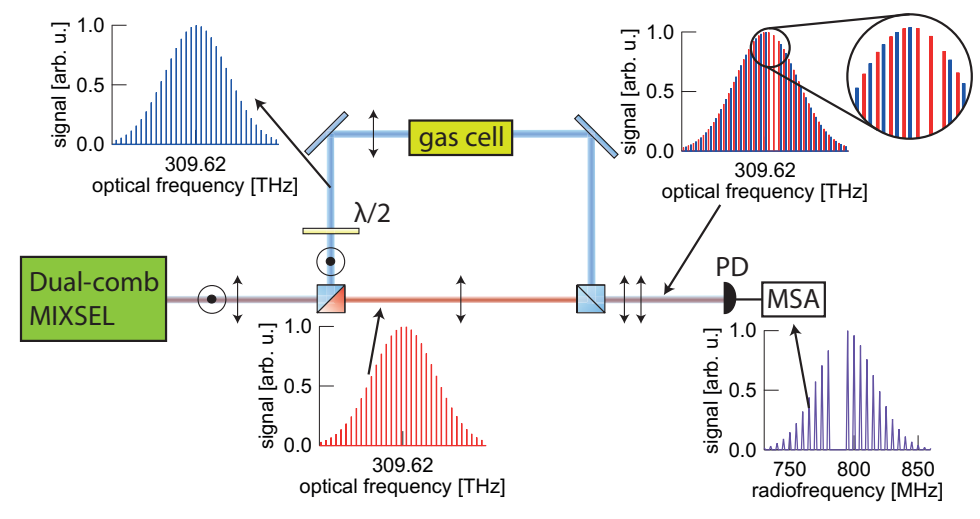

Figure 3.2: Potential gas sensing application using a dual comb MIXSEL: The two modelocked beams are separated with a polarizing beam splitter and one of the beams is guided through a gas cell. Both beams are then superimposed on a photo detector (PD) and measured with a microwave spectrum analyzer (MSA).

microwave frequency comb (Fig. 3.2). Another advantage of our systems is the fact that both output beams are collinear, fully overlapping and can be separated by a simple polarizing beam splitter. This typically simplifies potential applications such as pump-probe experiments for example. With the difference in pulse repetition frequency $\Delta f_{\text {rep }}$ of the two beams a total measurement time window $T=1 / f_{\text {rep }}$ can be scanned with a step increment size $\Delta f_{\text {rep }} / f_{\text {rep }}^{2}$ without using any mechanical delay line (Fig. 3.3).

\subsubsection{Experimental setup}

The MIXSEL chip used for this experiment was grown with a VEECO GEN III (Veeco instruments Inc.) molecular beam epitaxy machine. It consists of an anti-reflection section, followed by the active gain region, which contains $7 \mathrm{InGaAs}$ quantum wells (Fig. 3.4). Between the gain region and the InAs quantum dot absorber layer we inserted a distributed Bragg distributed Bragg reflector (DBR) for the pump light to prevent any bleaching of the saturable absorber layer by the pump light. The absorber is followed by a DBR for the laser wavelength. The detailed 


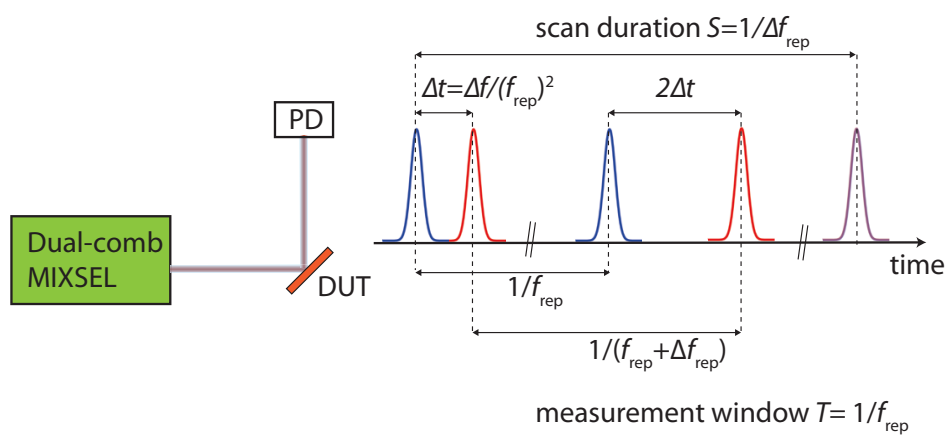

Figure 3.3: Potential pump-probe measurement without a mechanical delay line using a dual comb MIXSEL: device under test (DUT) in reflection (shown here) or transmission simply using a PD. The difference in pulse repetition frequency allows for a time dependent measurement.

parameters of the saturable absorber, for example the modulation depth and the saturation fluence, can be found in Ref [29]. The structure is grown in a single epitaxial growth run in reverse order and is then flipchip bonded to a $600-\mu \mathrm{m}$ thick diamond heat spreader [30]. The GaAs substrate is removed subsequently by chemical wet etching. For more details on the MIXSEL design we refer to Ref [22].

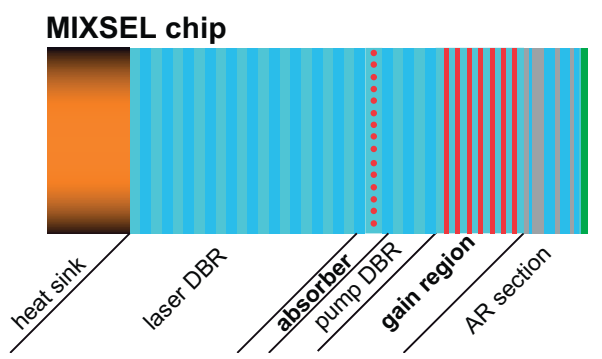

Figure 3.4: Structure of the MIXSEL chip: an anti-reflection (AR) section is followed by the gain region containing 7 InGaAs quantum wells. The InAs quantum dot saturable absorber is placed between the distributed Bragg reflector (DBR) for the laser light and the pump light. The chip is flip-chip bonded to a diamond heat spreader. 
Since the saturable absorber and the gain region are integrated into a single chip, the MIXSEL can be modelocked in a simple straight cavity, with only the MIXSEL chip and a curved output coupler (OC) as the two end mirrors. We then inserted a calcium carbonate $\left(\mathrm{CaCO}_{3}\right)$ birefringent crystal into the cavity to spatially separate the one cavity beam into two collinear but spatially separated beams with perpendicular polarizations on the MIXSEL chip (Fig. 3.5). We use $\mathrm{CaCO}_{3}$ due to its relative high refractive index contrast of 0.165 at $960 \mathrm{~nm}$ and its low group delay dispersion (GDD) of $52 \mathrm{fs}^{2} / \mathrm{mm}$ and $33 \mathrm{fs}^{2} / \mathrm{mm}$ along the extraordinary and the ordinary axes, respectively [31]. The length of the crystal is $2 \mathrm{~mm}$, which leads to a spatial separation of the cross-polarized beams of $210 \mu \mathrm{m}$. The beam diameter of the individual beams on the MIXSEL chip is estimated to be $220 \mu \mathrm{m}$. In addition, an anti-reflection coated 20- $\mu \mathrm{m}$ thick fused silica etalon is placed between the crystal and the OC for wavelength tuning. We chose a small angle of incidence to reduce polarization-dependent effects.

Both cavity spots on the MIXSEL are pumped at $808 \mathrm{~nm}$ with the same multi-mode $\left(\mathrm{M}^{2} \approx 36\right)$ fiber-coupled laser-diode array. We used two different pump configurations that worked equally well. In one configuration the collimated pump beam is split by a $50: 50$ beam splitter and both beams are separately focused onto the two cavity spots on the chip (Fig. 3.5). In another configuration the pump beam shape is adapted with cylindrical lenses to have an elliptical beam shape covering both cavity spots. The first configuration offers more flexibility on the position and size of the pump spots. It also improves the efficiency due to a better overlap of the pumped region with the high-field optical gain region. On the other hand the second configuration is more compact and requires less optical elements.

The cavity and pump optics are placed inside a closed aluminum housing to prevent disturbing airflow. All optical elements are mounted directly on the base plate to minimize mechanical vibrations (Fig. 3.5(a)).

The two orthogonally polarized pulse trains are coupled out collinearly with an OC of $0.5 \%$ transmission and a radius of curvature of $100 \mathrm{~mm}$ and are then separated by a polarizing beam splitter (PBS) (Fig. 3.5(b)). To allow for optical interference of the two pulse trains, the polarization of one 


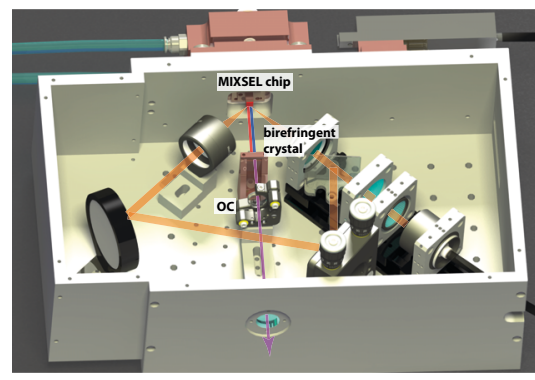

a)

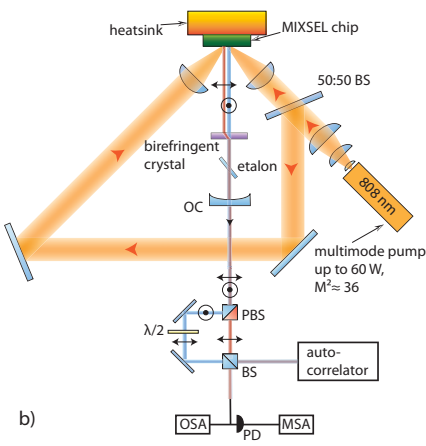

Figure 3.5: a) A design image of the laser housing with the optically pumped dual comb MIXSEL. The linear straight cavity is formed by the MIXSEL chip end mirror and the output coupler (OC). An intracavity birefringent crystal is used to generate the two combs. One of the two pumping configuration is shown here with the orange path of the pump light. An aluminum housing surrounds the cavity and all optical elements are mounted directly on the base plate to minimize mechanical vibrations. b) Schematic of the setup, showing the paths of the two modelocked beams, p-polarized in red and s-polarized in blue. Again the pump beam is shown in orange. PBS: polarizing beam splitter, BS: beam splitter, PD: photo detector, OSA: optical spectrum analyzer, MSA: microwave spectrum analyzer, $\lambda / 2$ : halfwave plate

of the beams is turned by $90^{\circ}$ with a half-wave plate and then both beams are collinearly combined by a non-polarizing beam splitter (BS). By superposition in the optical domain and detecting the combined beams on a photo detector all possible beat notes can be measured in the microwave frequency domain. Furthermore the optical spectrum, the microwave spectrum and the autocorrelation of each individual pulse train are recorded.

\subsubsection{Experimental results}

At a total pump power of $10 \mathrm{~W}$, divided equally for pumping the separate laser beams, both cross-polarized pulse trains show stable and self-starting fundamental modelocking (i.e. only one pulse per cavity roundtrip). The temperature of the MIXSEL chip is stabilized with a Peltier element to $15^{\circ} \mathrm{C}$. The modelocking results are shown in Fig. 3.6 and Table 3.1. With $1.890 \mathrm{GHz}$, the pulse repetition frequency of the p-polarized beam is $5 \mathrm{MHz}$ lower than the s-polarized beam due to the slightly longer opti- 
cal path length in the birefringent crystal. The cavity length was chosen for a pulse repetition frequencies around $2 \mathrm{GHz}$. However, higher repetition rates up to $10 \mathrm{GHz}$ are feasible with the same MIXSEL structure [29]. The separation of the individual beams on the MIXSEL is $210 \mu \mathrm{m}$. The individual Gaussian beam diameters of approximately $220 \mu \mathrm{m}$ gives only a small overlap of the two laser beams in the tail of their Gaussian beam profiles. Figure 3.6(a) and Fig. 3.6(b) show the optical spectra of the spolarized and the p-polarized beam, respectively. The center wavelengths around $966 \mathrm{~nm}$ exhibit a small difference, but the two spectra show a sufficiently strong overlap. The $2^{n d}$ harmonic intensity autocorrelations of the two beams are displayed in Fig. 3.6(c) and Fig. 3.6(d) with pulse durations of $13.5 \mathrm{ps}$ and $19.1 \mathrm{ps}$, respectively. The different pulse durations of the two beams originate from the spatial separation on the MIXSEL chip, because the semiconductor growth of the MIXSEL chip is not perfectly homogeneous. In addition, the pulses are chirped because the GDD for the MIXSEL chip was not optimized [22]. Previously we have shown that better GDD compensation can generate much shorter and close to transform-limited pulses [23].

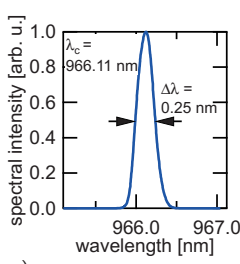

a)

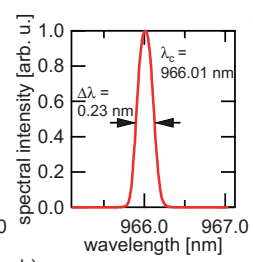

b)

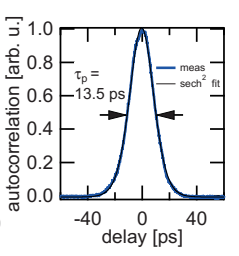

c)

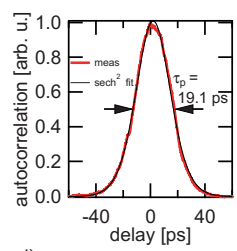

d)

Figure 3.6: Modelocking results of the dual comb MIXSEL: a) Optical spectrum of the s-polarized pulse train; b) Optical spectrum of the p-polarized pulse train; c) $2^{\text {nd }}$ harmonic intensity autocorrelation of the s-polarized beam; d) $2^{\text {nd }}$ harmonic intensity autocorrelation of the p-polarized beam

The electric field $E_{\mathrm{k}}$ of both pulse trains in time with $k=1,2$ can be 
Table 3.1: Modelocking parameters of the two pulse trains

\begin{tabular}{l|c|c}
\hline & s-polarized beam (blue) & p-polarized beam (red) \\
\hline output power $P_{\text {out }}[\mathrm{mW}]$ & 78 & 70 \\
pulse repetition frequency $f_{\text {rep }}[\mathrm{GHz}]$ & 1.895 & 1.890 \\
center wavelength $\lambda_{\text {center }}[\mathrm{nm}]$ & 966.11 & 966.01 \\
spectral bandwidth FWHM [nm] & 0.25 & 0.23 \\
pulse duration $\tau_{\mathrm{p}}[\mathrm{ps}]$ & 13.5 & 19.1 \\
time-bandwidth-product TBP & $1.07\left(3.3 \times \mathrm{sech}^{2}\right)$ & $1.39\left(4.35 \times \mathrm{sech}^{2}\right)$ \\
\hline
\end{tabular}

written as

$$
\begin{aligned}
E_{\mathrm{k}}(t) & =\left(A_{\mathrm{k}}(t) \exp \left(2 \pi i f_{\text {center, } \mathrm{k}} t\right)\right) *\left[\sum_{\mathrm{n}} \delta\left(t-n \frac{1}{f_{\text {rep }, \mathrm{k}}}\right) \exp \left(2 \pi i f_{\text {CEO, } \mathrm{k}} t\right)\right] \\
& + \text { c.c., }
\end{aligned}
$$

with $A_{\mathrm{k}}(t)$ the pulse envelope

$$
A_{\mathrm{k}}(t)=\operatorname{sech}\left(\frac{t}{\tau_{k}}\right)
$$

The Fourier transformation of [Eq. (3.1)] gives the expression for the pulse trains in the frequency domain

$$
\tilde{E}_{\mathrm{k}}(f)=\tilde{A}_{\mathrm{k}}\left(f-f_{\text {center }, k}\right) \sum_{\mathrm{n}} \delta\left(f-n f_{\mathrm{rep}, k}-f_{\mathrm{CEO}, k}\right)+\text { c.c., }
$$

where the pulse envelopes are normalized accordingly. The center frequency $f_{\text {center,k }}$ is given for both beams by the carrier-envelope-offset frequency $f_{\mathrm{CEO}, \mathrm{k}}$ plus a multiple $m_{\mathrm{k}}$ of the pulse repetition frequency $f_{\text {rep,k }}$

$$
f_{\text {center }, \mathrm{k}}=f_{\mathrm{CEO}, \mathrm{k}}+m f_{\text {rep, } \mathrm{k}}
$$

The difference $\Delta f_{\text {rep }}$ between the pulse repetition frequencies of the two beams arises from the difference in the optical path length in the birefringent crystal, leading to

$$
f_{\text {rep }, 2}=f_{\text {rep }, 1}+\Delta f_{\text {rep }}
$$

The photo detector (PD) measures then a photo current IPD, which is

$$
I_{\mathrm{PD}}=\left|E_{1}(t)+E_{2}(t)\right|^{2}
$$


The microwave spectrum analyzer (MSA) then measures the frequencyresolved power of this $\mathrm{PD}$ signal, which is proportional to $I_{\mathrm{PD}}^{2}$. Thus the Fourier transformation (FT) determines the measured microwave spectrum $S_{\text {freq }}$ :

$$
S_{\text {freq }}=F T\left(\left|E_{1}(t)+E_{2}(t)\right|^{4}\right)
$$

With [Eq. (3.1)-(3.7)] we simulated the expected microwave spectrum using as an input the measured pulse durations, center wavelengths, and pulse repetition rates of the two pulse trains. The simulated microwave spectrum (Figs. 3.7(f)-3.7(j)) shows excellent agreement with the measurements (Figs. 3.7(a)-3.7(e)). All features of the microwave spectrum are clearly visible for all measured higher harmonics (Fig. 3.7(a)). The drop of the harmonic signal above $5 \mathrm{GHz}$ is due to the limited amplifier bandwidth after the PD before the MSA.

Between DC and the pulse repetition frequencies, two comb structures $\left(\mathrm{comb}_{1}\right.$ and $\left.\mathrm{comb}_{2}\right)$ are visible (Fig. 3.7(b)). The center frequency of the first comb $f_{\text {comb }_{1} \text {,center }}$ is given by:

$$
\begin{aligned}
f_{\mathrm{comb}_{1, \text { center }}} & =\left(f_{\text {center }, 2}-f_{\text {center }, 1}\right) \bmod f_{\text {rep }, 1} \\
& =\left(\left(f_{\mathrm{CEO}, 2}+m_{2} f_{\text {rep }, 2}\right)-\left(f_{\mathrm{CEO}, 1}+m_{1} f_{\text {rep }, 1}\right)\right) \bmod f_{\text {rep }, 1} \\
& =\left(\Delta f_{\mathrm{CEO}}+\left(m_{2}-m_{1}\right) f_{\text {rep }, 1}+m_{2} \Delta f_{\text {rep }}\right) \bmod f_{\text {rep }, 1} \\
& =\left(\Delta f_{\mathrm{CEO}}+m_{2} \Delta f_{\text {rep }}\right) \bmod f_{\text {rep }, 1}
\end{aligned}
$$

where "mod" is the modulus-function. The spacing of the comb lines is set by $\Delta f_{\text {rep }}$ (Fig. 3.7(c)), set by the length of the birefringent crystal. Since both lasers are not stabilized in repetition frequency in this measurement, slight drifts are causing deviations from the exact difference in repetition frequency. These microwave combs, comb ${ }_{1}$ (green in Fig. 3.7(b) and Fig. 3.7(c)) and $\mathrm{comb}_{2}$, result from interference between the optical frequencies of the two modelocked laser beams and represent a direct link between the optical frequencies and the electronically accessible microwave frequencies. They are only visible if the two optical spectra overlap and if both beams are detected in the same polarization. On the other hand, the pulse repetition frequencies $f_{\text {rep }}$ p-pol and $f_{\text {rep }}$ s-pol (Fig. 3.7(d)) and the difference in pulse repetition frequencies $\Delta f_{\text {rep }}$ or multiple of $\Delta f_{\text {rep }}$ (Fig. 3.7(e)) are independent of the polarization and can be measured in 


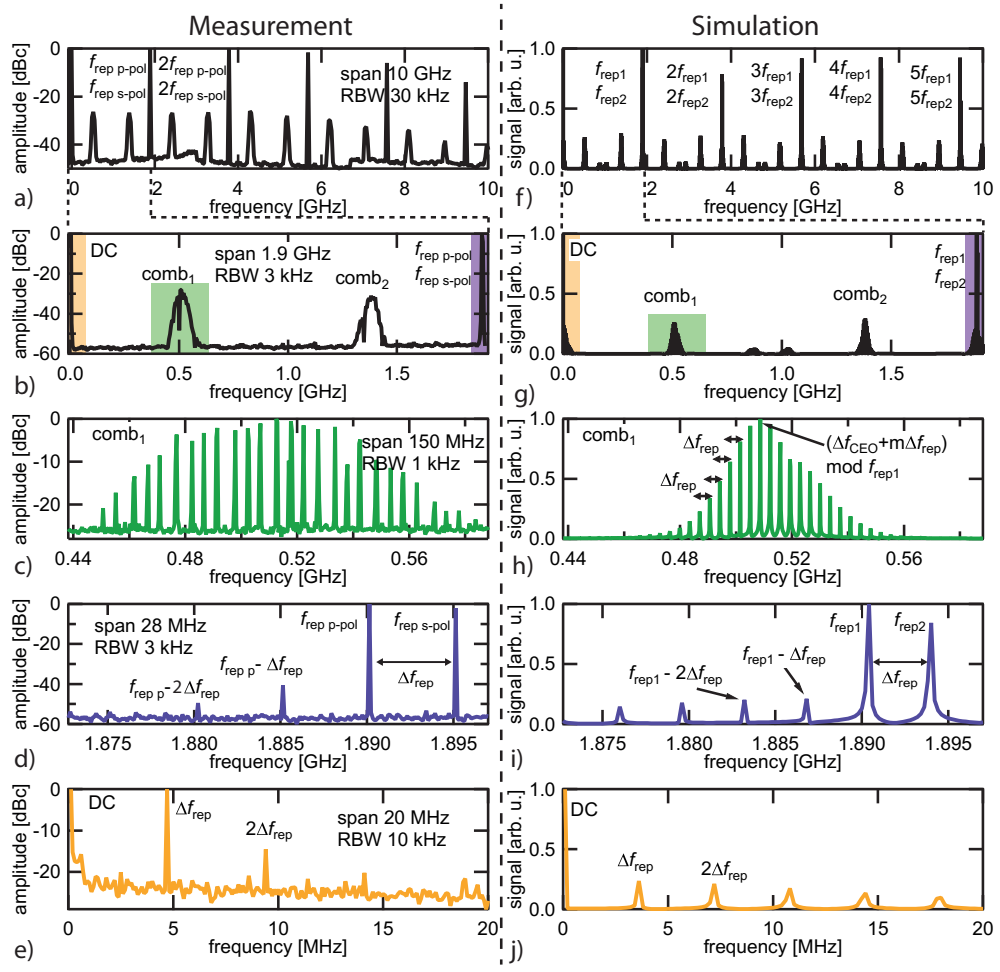

Figure 3.7: a) Microwave spectrum analyzer (MSA) signal from DC to $10 \mathrm{GHz}$ showing the pulse repetition frequencies and their higher harmonics as well as the beat-combs. The drop of the harmonic signal above $5 \mathrm{GHz}$ is due to the limited bandwidth of the amplifier used to amplify the signal after the photo detector (PD) before the MSA. b) Zoom-in from DC to $1.9 \mathrm{GHz}$ with a resolution bandwidth (RBW) of $3 \mathrm{kHz}$. c) Zoom-in around the first comb with a span of $150 \mathrm{MHz}$ and a RBW of $1 \mathrm{kHz}$. d) Zoom-in around the pulse repetition frequencies with a span of $28 \mathrm{MHz}$ and a RBW of $3 \mathrm{kHz}$. e) Zoom-in around DC with a span of $20 \mathrm{MHz}$ and a RBW of $10 \mathrm{kHz}$. $\mathrm{f}-\mathrm{j}$ ) Simulated microwave spectrum in the corresponding frequency spans; In g) "mod" means modulo. 
cross-polarized detection as well as in the same polarization. In the simulation (Fig. 3.7(f)) two additional combs are visible, which result from the beat between the two combs comb 1 and comb 2 . They are not visible in the measurement (Fig. 3.7(b)) because they are below the noise level. The amplitudes of the frequencies in the simulation (Figs. 3.7(a)-3.7(e)) differ from the amplitudes of the frequencies in the measurement (Figs. 3.7(f)3.7(j)) because the simulation is on a linear scale and the measurement is on a logarithmic scale.
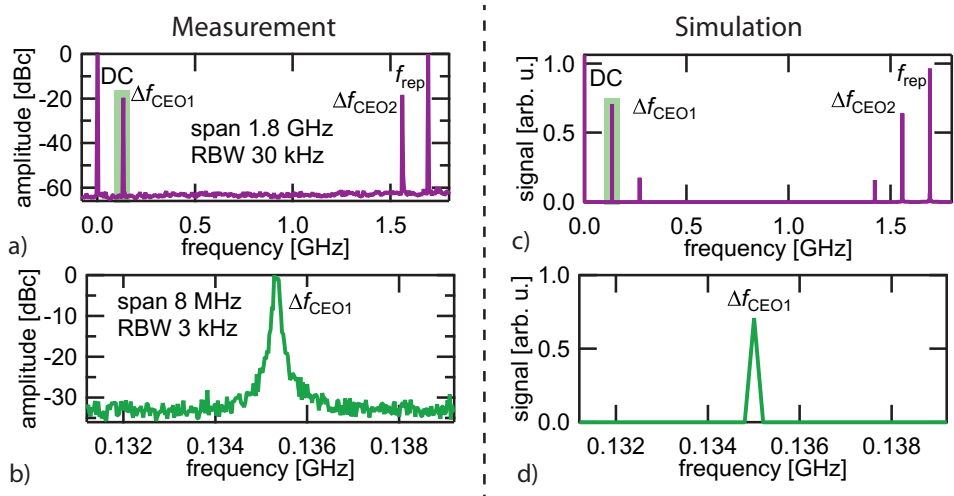

Figure 3.8: a) Measured microwave spectrum showing both relative CEO frequencies $\Delta f_{\mathrm{CEO} 1}$ and $\Delta f_{\mathrm{CEO} 2}$ and the pulse repetition frequency $f_{\text {rep. }}$ b) Zoom-in around $\Delta f_{\mathrm{CEO} 1}$ with a frequency span of $8 \mathrm{MHz}$ and a reduced RBW of $3 \mathrm{kHz}$. c) Simulated microwave spectrum showing both relative CEO frequencies $\Delta f_{\mathrm{CEO} 1}$ and $\Delta f_{\mathrm{CEO} 2}$ and the repetition frequency $f_{\text {rep }}$ and the beat between the two relative CEO frequencies. d) Simulated $\Delta f_{\mathrm{CEO} 1}$ in an $8 \mathrm{MHz}$ span.

The position of the first comb (Fig. 3.7(g)) depends directly on the difference between the CEO frequencies $\Delta f_{\text {CEO }}$ of the two beams. This becomes more obvious when both beams have exactly the same pulse repetition frequency. For an experimental demonstration we add a second identical birefringent calcite crystal between the first crystal and the MIXSEL chip with the same thickness but with the optical axis rotated by $90^{\circ}$ with respect to the first crystal. The thickness of each of the crystals is chosen in this configuration to give a separation of $150 \mu \mathrm{m}$ in order to 
have approximately the same total separation of $\approx 210 \mu \mathrm{m}$ of the two cavity spots as in the previous configuration. This leads to two modelocked cross-polarized beams with identical pulse repetition frequencies. In this case, $\Delta f_{\text {rep }}=0$ in [Eq. (3.8)] and the interference between the optical frequencies of the two beams results in two strong relative $\mathrm{CEO}$ frequencies $\Delta f_{\mathrm{CEO} 1}$ and $\Delta f_{\mathrm{CEO} 2}$ (Fig. 3.8(a)). A zoom-in around $\Delta f_{\mathrm{CEO} 1}$ with a frequency span of $8 \mathrm{MHz}$ and a reduced resolution bandwidth (RBW) of $3 \mathrm{kHz}$ is shown in Fig. 3.8(b). Again, the simulations agree very well with the measurements (Figs. 7(c) and 7(d)). The additional peaks in the simulation (Fig. 3.8(c)) result from a beat between $\Delta f_{\mathrm{CEO} 1}$ and $\Delta f_{\mathrm{CEO} 2}$. They are below the noise level in the measurement (Fig. 3.8(a)).

We were able to simultaneously stabilize the different pulse repetition frequency in both output beams of the dual comb MIXSEL and measured the resulting phase noise with a signal source analyzer. In stabilized operation a noise reduction of up to $100 \mathrm{~dB} / \mathrm{Hz}$ for frequencies below $100 \mathrm{~Hz}$ compared to free-running operation is achieved (Fig. 3.9). Further details on the feedback mechanism and stabilization dynamics will be discussed in the near future and is beyond the scope of this paper.

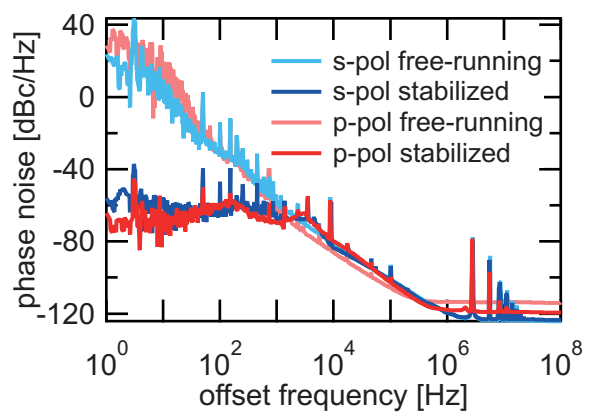

Figure 3.9: Phase noise of both beams in free-running and stabilized operation, measured with a signal source analyzer. 


\subsubsection{Conclusion and outlook}

We have presented a novel straightforward technique of generating two fundamentally modelocked pulse trains within the same laser cavity. We utilized the key advantage of the MIXSEL, having the saturable absorber integrated into the gain chip, to enable a compact design with a linear straight cavity. We simultaneously stabilized the different pulse repetition rates of the two output beams of the dual comb MIXSEL. The beat signal of the optical interference of the two pulse trains detected with a photo detector (PD) and a microwave spectrum analyzer (MSA) provides a strikingly simple setup to down-convert terahertz optical frequencies into the electronically accessible microwave regime. This experiment offers for the first time access to the relative CEO frequencies of an SDL. We will further study the dynamics of the $\mathrm{CEO}$ frequencies and possible stabilization mechanisms. Our first proof-of-principle concept experiments were performed with a MIXSEL structure that only supports rather long picosecond pulses. However recently femtosecond operation of a MIXSEL has been demonstrated [23]. In the near future the experiments will be performed with a MIXSEL structure that generates shorter pulses with a much larger optical bandwidth and therefore also a larger microwave beat comb. This will make the dual-comb MIXSEL a very compact and inexpensive source for applications in frequency metrology, optical sensing or pump-probe experiments for example.

Acknowledgements The authors acknowledge support of the technology and clean room facility FIRST of ETH Zurich for advanced micro- and nanotechnology. This work was financed by the Swiss Confederation Program Nano-Tera.ch, which was scientifically evaluated by the Swiss National Science Foundation (SNSF).

\subsubsection{References and links}

1. F. Keilmann, C. Gohle, and R. Holzwarth, "Time-domain midinfrared frequency-comb spectrometer," Opt. Lett. 29(13), 1542-1544 (2004).

2. A. Schliesser, M. Brehm, F. Keilmann, and D. van der Weide, 
"Frequency-comb infrared spectrometer for rapid, remote chemical sensing," Opt. Express 13(22), 9029-9038 (2005).

3. S. Schiller, "Spectrometry with frequency combs," Opt. Lett. 27(9), 766-768 (2002).

4. I. Coddington, W. C. Swann, and N. R. Newbury, "Coherent multiheterodyne spectroscopy using stabilized optical frequency combs," Phys. Rev. Lett. 100(1), 013902 (2008).

5. B. Bernhardt, A. Ozawa, P. Jaquet, M. Jacquey, Y. Kobayashi, T. Udem, R. Holzwarth, G. Guelachvili, T. Hänsch, and N. Piqué, "Cavity-enhanced dual-comb spectroscopy," Nat. Photonics 4(1), 5557 (2010).

6. A. Bartels, R. Cerna, C. Kistner, A. Thoma, F. Hudert, C. Janke, and T. Dekorsy, “Ultrafast time-domain spectroscopy based on high-speed asynchronous optical sampling," Rev. Sci. Instrum. 78(3), 035107 (2007).

7. M. Kuznetsov, F. Hakimi, R. Sprague, and A. Mooradian, "Highpower $(>0.5-\mathrm{W} \mathrm{CW}$ ) diode-pumped vertical-external-cavity surfaceemitting semiconductor lasers with circular TEM00 beams," IEEE Photon. Technol. Lett. 9(8), 1063-1065 (1997).

8. S. Calvez, J. E. Hastie, M. Guina, O. G. Okhotnikov, and M. D. Dawson, "Semiconductor disk lasers for the generation of visible and ultraviolet radiation," Laser Photon. Rev. 3(5), 407-434 (2009).

9. B. Rösener, M. Rattunde, R. Moser, S. Kaspar, C. Manz, K. Köhler, and J. Wagner, "GaSb-based optically pumped semiconductor disk lasers emitting in the 2.0-2.8 $\mu \mathrm{m}$ wavelength range," Proc. SPIE 7578, 75780X (2010).

10. D. J. M. Stothard, J.-M. Hopkins, D. Burns, and M. H. Dunn, “Stable, continuous-wave, intracavity, optical parametric oscillator pumped by a semiconductor disk laser (VECSEL)," Opt. Express 17(13), 10648-10658 (2009).

11. J. D. Berger, D. W. Anthon, A. Caprara, J. L. Chilla, S. V. Govorkov, A. Y. Lepert, W. Mefferd, Q.-Z. Shu, and L. Spinelli, "20 Watt CW 
TEM00 intracavity doubled optically pumped semiconductor laser at $532 \mathrm{~nm}, "$ Proc. SPIE 8242, 824206 (2012).

12. H. Kahle, R. Bek, M. Heldmaier, T. Schwarzbäck, M. Jetter, and P. Michler, "High optical output power in the UVA range of a frequency-doubled, strain-compensated AlGaInP-VECSEL," Appl. Phys. Express 7(9), 092705 (2014).

13. U. Keller, K. J. Weingarten, F. X. Kärtner, D. Kopf, B. Braun, I. D. Jung, R. Fluck, C. Hönninger, N. Matuschek, and J. Aus der Au, "Semiconductor saturable absorber mirrors (SESAMs) for femtosecond to nanosecond pulse generation in solid-state lasers," IEEE J. Sel. Top. Quantum Electron. 2(3), 435-453 (1996).

14. U. Keller, "Recent developments in compact ultrafast lasers," Nature 424(6950), 831-838 (2003).

15. U. Keller and A. C. Tropper, "Passively modelocked surface-emitting semiconductor lasers," Phys. Rep. 429(2), 67-120 (2006).

16. S. Hoogland, S. Dhanjal, A. C. Tropper, S. J. Roberts, R. Häring, R. Paschotta, and U. Keller, "Passively mode-locked diode-pumped surface-emitting semiconductor laser," IEEE Photon. Technol. Lett. 12(9), 1135-1137 (2000).

17. P. Klopp, U. Griebner, M. Zorn, and M. Weyers, “Pulse repetition rate up to $92 \mathrm{GHz}$ or pulse duration shorter than $110 \mathrm{fs}$ from a modelocked semiconductor disk laser," Appl. Phys. Lett. 98(7), 071103 (2011).

18. K. G. Wilcox, A. C. Tropper, H. E. Beere, D. A. Ritchie, B. Kunert, B. Heinen, and W. Stolz, " $4.35 \mathrm{~kW}$ peak power femtosecond pulse mode-locked VECSEL for supercontinuum generation," Opt. Express 21(2), 1599-1605 (2013).

19. H. R. Telle, G. Steinmeyer, A. E. Dunlop, J. Stenger, D. H. Sutter, and U. Keller, "Carrier-envelope offset phase control: A novel concept for absolute optical frequency measurement and ultrashort pulse generation," Appl. Phys. B 69(4), 327-332 (1999).

20. C. A. Zaugg, A. Klenner, M. Mangold, A. S. Mayer, S. M. Link, F. Emaury, M. Golling, E. Gini, C. J. Saraceno, B. W. Tilma, and U. 
Keller, "Gigahertz self-referenceable frequency comb from a semiconductor disk laser," Opt. Express 22(13), 16445-16455 (2014).

21. D. J. H. C. Maas, A.-R. Bellancourt, B. Rudin, M. Golling, H. J. Unold, T. Südmeyer, and U. Keller, "Vertical integration of ultrafast semiconductor lasers," Appl. Phys. B 88(4), 493-497 (2007).

22. B. Rudin, V. J. Wittwer, D. J. H. C. Maas, M. Hoffmann, O. D. Sieber, Y. Barbarin, M. Golling, T. Südmeyer, and U. Keller, "High-power MIXSEL: an integrated ultrafast semiconductor laser with $6.4 \mathrm{~W}$ average power," Opt. Express 18(26), 27582-27588 (2010).

23. M. Mangold, V. J. Wittwer, C. A. Zaugg, S. M. Link, M. Golling, B. W. Tilma, and U. Keller, "Femtosecond pulses from a modelocked integrated external-cavity surface emitting laser (MIXSEL)," Opt. Express 21(21), 24904-24911 (2013).

24. M. Mangold, C. A. Zaugg, S. M. Link, M. Golling, B. W. Tilma, and U. Keller, "Pulse repetition rate scaling from 5 to $100 \mathrm{GHz}$ with a high-power semiconductor disk laser," Opt. Express 22(5), 6099-6107 (2014).

25. M. Mangold, S. M. Link, A. Klenner, C. A. Zaugg, M. Golling, B. W. Tilma, and U. Keller, "Amplitude Noise and Timing Jitter Characterization of a High-Power Mode-Locked Integrated External-Cavity Surface Emitting Laser," IEEE Photonics J. 6(1), 1-9 (2014).

26. G. Baili, L. Morvan, M. Alouini, D. Dolfi, F. Bretenaker, I. Sagnes, and A. Garnache, "Experimental demonstration of a tunable dualfrequency semiconductor laser free of relaxation oscillations," Opt. Lett. 34(21), 3421-3423 (2009).

27. F. A. Camargo, J. Barrientos, G. Baili, L. Morvan, D. Dolfi, D. Holleville, S. Guerandel, I. Sagnes, P. Georges, and G. Lucas-Leclin, "Coherent dual-frequency emission of a vertical external-cavity semiconductor laser at the cesium D2 line," IEEE Photon. Technol. Lett. 24(14), 1218-1220 (2012).

28. I. Znakovskaya, E. Fill, N. Forget, P. Tournois, M. Seidel, O. Pronin, F. Krausz, and A. Apolonski, "Dual frequency comb spectroscopy with a single laser," Opt. Lett. 39(19), 5471-5474 (2014). 
29. V. J. Wittwer, M. Mangold, M. Hoffmann, O. D. Sieber, M. Golling, T. Sudmeyer, and U. Keller, "High-power integrated ultrafast semiconductor disk laser: multi-Watt $10 \mathrm{GHz}$ pulse generation," Electron. Lett. 48(18), 1144-1145 (2012).

30. R. Häring, R. Paschotta, A. Aschwanden, E. Gini, F. Morier-Genoud, and U. Keller, "High-power passively mode-locked semiconductor lasers," IEEE J. Quantum Electron. 38(9), 1268-1275 (2002).

31. G. Ghosh, "Dispersion-equation coefficients for the refractive index and birefringence of calcite and quartz crystals," Opt. Commun. 163(1-3), 95-102 (1999). 



\section{Noise analysis of dual-comb modelocked lasers}

The results of chapter 2 show that the already good timing phase noise performance of a free-running MIXSEL can be drastically improved by actively stabilizing the pulse repetition rate via cavity length adjustments with a piezo-controlled output coupler. The dual-comb method presented in chapter 3 delivers two modelocked pulse trains from a single MIXSEL chip with slightly different pulse repetition rates. In this chapter we apply the active feedback loop of chapter 2 to the dual-comb MIXSEL to find out how both pulse repetition rates behave if one of them is actively stabilized. The initially surprising result that the cavity length adjustments to correct the error signal of one beam did not significantly affect the other beam (i.e. the timing jitter noise was uncorrelated), even though they share all components within the linear cavity, is presented in section 4.1.2. One important step to understand the underlying physics and to explain the uncorrelated timing jitter noise was to separate the gain and the absorber dynamics. Since this is not possible in a MIXSEL, we applied the polarizationduplexing method to a SESAM-modelocked neodymium-doped yttrium aluminium garnet $\left(\mathrm{Nd}: \mathrm{Y}_{3} \mathrm{Al}_{5} \mathrm{O}_{12}\right)$ (Nd:YAG) laser in section 4.1.3. This dual-comb Nd:YAG laser shows that the dual-comb method is also applicable to SESAM-modelocked diode-pumped solid-state lasers. With a thorough analysis of the two dual-comb lasers in section 4.1.4 the uncorrelated behavior of the two beams can be explained. 
The dual-comb noise analysis study is presented in the following Optics Express publication [36]. (๑) 2016 Optical Society of America. One print or electronic copy may be made for personal use only. Systematic reproduction and distribution, duplication of any material in this paper for a fee or for commercial purposes, or modifications of the content of this paper are prohibited.)

\title{
4.1 Dual-comb modelocked lasers: semiconductor sat- urable absorber mirror decouples noise stabilization
}

\author{
Sandro M. Link, Alexander Klenner, and Ursula Keller
}

Department of Physics, Institute for Quantum Electronics, ETH Zürich, 8093 Zürich, Switzerland

In this paper we present the stabilization of the pulse repetition rate of dual-comb lasers using an intracavity semiconductor saturable absorber mirror (SESAM) for passive modelocking and an intracavity birefringent crystal for polarization-duplexing to obtain simultaneous emission of two modelocked beams from the same linear cavity sharing all components. Initially surprising was the observation that the cavity length adjustments to stabilize one polarization did not significantly affect the pulse repetition rate of the other. We gained insight in the underlying physics using both a semiconductor and Nd:YAG laser gain material with the conclusion that the pulse arrival timing jitter of the two beams is decoupled by the uncorrelated time delay from the saturated SESAM and becomes locked with sufficient but not too much pulse overlap. Noise stabilization is in all cases still possible for both combs. The dual-comb modelocked laser is particularly interesting for the semiconductor laser enabling the integration of gain and absorber layers within one wafer (referred to as the modelocked integrated external-cavity surface emitting laser - MIXSEL). 


\subsubsection{Introduction}

A modelocked laser can support not only very short pulses but at the same time very precise frequency metrology for the most accurate clocks. These ultrafast - or ultra-short pulse - lasers are dramatically impacting many areas of photonics, from basic science to industrial manufacturing and biomedicine. The design and performance of the lasers behind these applications is critical for new discoveries, creating new applications and opening new market opportunities. Modelocked lasers produce a frequency comb for which the frequency spacing (i.e. the pulse repetition rate) has been stabilized in the 1980s [1,2] achieving close to quantum-noise limited performance with diode-pumped solid-state lasers [3]. However the stabilization of the frequency comb offset (i.e. the carrier envelope offset (CEO) frequency) remained a challenge and only has become possible more recently [4-6]. Since then the field of optical frequency combs has evolved very quickly, and current applications range from high-precision spectroscopy $[7,8]$ over frequency metrology $[4,9]$ to ultra-high-speed optical communication $[10,11]$. Many of these applications are based not only on one, but on two frequency combs such as dual-comb spectroscopy [1216], asynchronous optical sampling (ASOPS) [17], pump probe measurements and fiber Bragg grating sensing [18]. Usually two separate lasers need to be stabilized and therefore, one simple, compact and cost-efficient dual-comb laser would be greatly beneficial for these kind of applications.

We have introduced the concept of dual-comb lasers [19] to generate two modelocked beams with slightly different pulse repetition rates using only a single gain medium in a shared cavity. The dual-comb emission is obtained by inserting a birefringent crystal into the laser cavity, to split an unpolarized cavity-beam into two cross-polarized beams, which are collinear but spatially separated and have slightly different optical path lengths in the birefringent crystal (Fig. 4.1). The pulse repetition rate is set by the cavity length and the difference by the different optical path length inside the birefringent crystal (Fig. 4.1) that can be adjusted by the crystal thickness and even compensated using a second birefringent crystal. For the first dual-comb laser demonstration [19] we used an optically pumped semiconductor laser, referred to as the modelocked integrated external-cavity surface emitting laser (MIXSEL) [20], which integrates the 


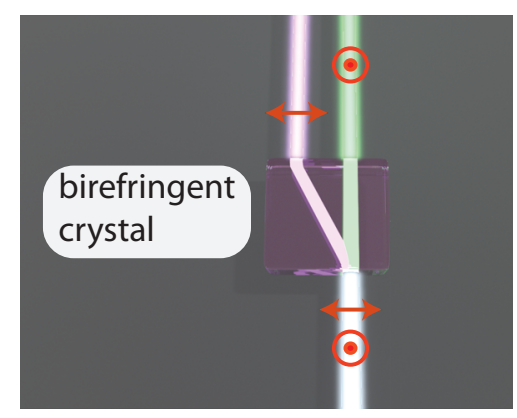

Figure 4.1: A birefringent crystal splits an unpolarized beam into two orthogonally polarized beams which are collinear but spatially separated with different optical path lengths in the birefringent crystal.

saturable absorber of a semiconductor saturable absorber mirror (SESAM) $[21,22]$ with the gain structure of a vertical external-cavity surface emitting laser (VECSEL) [23] into a single semiconductor wafer. Thus modelocking in a simple straight linear cavity can be achieved with a MIXSEL (Fig. 4.2). VECSELs and MIXSELs are part of the family of optically pumped semiconductor disk lasers and have become successful industrial products due to their large spectral range of operation from the infrared to the visible [24-26] and even reaching the UV regime [27,28]. Passively modelocked VECSELs first demonstrated in 2000 [29,30] using SESAMs have been improved to peak power levels of up to $4.35 \mathrm{~kW}$ [31] and pulses as short as $107 \mathrm{fs}$ [32]. The potential of optically pumped semiconductor disk lasers for the generation of low-noise frequency combs has been demonstrated using a SESAM-modelocked VECSEL, however, at this point still with the additional complexity of external pulse amplification and compression to obtain the required peak power for frequency comb stabilization [33].

More recent progress with MIXSELs results in pulse durations as short as $253 \mathrm{fs}$ with $240 \mathrm{~W}$ of peak power [34]. The reduced complexity in comparison to any diode-pumped ultrafast solid-state laser makes the MIXSEL platform very attractive and particularly interesting for dual-comb lasers. The first demonstration of a dual-comb MIXSEL [19] generated two modelocked pulse trains with an average output power per beam of around $70 \mathrm{~mW}$, pulse durations of $13 \mathrm{ps}$ and 19 ps and a pulse repetition rate of 
$1.890 \mathrm{GHz}$ and $1.895 \mathrm{GHz}$, respectively.

A single-comb MIXSEL with actively stabilized pulse repetition frequency has shown excellent noise performance with record-low timing jitter for the arrival time of the pulses [35]. A dual-comb MIXSEL (Fig. 4.1 and 4.2), however, revealed an initially very surprising result: when the cavity length was corrected by the error signal of one beam only, the other beam was not affected significantly (i.e. the timing jitter noise was uncorrelated). This means that the pulse repetition rates of the two crosspolarized beams seem to be decoupled from each other even though they share all components within the linear cavity (Fig. 4.2).

In this paper we will explain the observed uncorrelated timing jitter noise of the two frequency combs (Fig. 4.3) and provide a solution how to stabilize both pulse repetition frequencies at the same time (Fig. 4.4). To explore the underlying physics we studied both a semiconductor and $\mathrm{Nd}$ :YAG dual-comb laser with opposite gain parameters in terms of upper state lifetimes (nanoseconds versus $230 \mu \mathrm{s}$ ) and gain cross sections $\left(\approx 10^{-14} \mathrm{~cm}^{2}\right.$ versus $\left.2.8 \times 10^{-19} \mathrm{~cm}^{2}\right)$ using in both cases the same pump laser diodes and the same intracavity birefringent crystals. For the dualcomb Nd:YAG laser (Fig. 4.5) we used a separate SESAM as one endmirror of the linear folded cavity which allowed us to have perfect beam overlap in the gain. The outcome was that the spatially and temporarily incoherent pump laser was not responsible for the uncorrelated noise observed with the dual comb MIXSEL because we observed the same behavior with the dual comb Nd:YAG laser using the same pumping schemes. Furthermore with the dual-comb Nd:YAG laser as shown in Fig. 4.5 we could separate the two beams on the SESAM alone. This gave us strong experimental evidence that the noise of both beams is uncorrelated due to the time delay, that is introduced by the saturated SESAM since only the leading edge of the pulse experiences absorption. This was further confirmed when the difference in pulse repetition rates was reduced to allow for sufficient temporal overlap of the two pulses on the SESAM. At a certain point for these small differences the two pulse repetition rates have become locked as long as we provide some partial spatial beam overlap on the SESAM. Furthermore, with an increasingly larger spatial overlap of the two beams on the SESAM, the modelocking has become only stable 
for one beam because of net-gain competition between the two beams due to small inhomogeneities in the birefringent crystal and SESAM.

For all cases we were able to finally stabilize both frequency combs. For applications such as high-precision spectroscopy we need the stabilization of both the pulse repetition frequency $f_{\text {rep }}$ (i.e. comb spacing) and of the carrier envelope offset (CEO) frequency $f_{\text {CEO }}$ (i.e. comb offset). The stabilization of the pulse repetition frequency of one of the beams enables for the first time to observe the effect on the phase noise of the other beam that shares the same cavity.

In the next sections we describe in more details the two different dual comb lasers and their noise characterizations. Our dual comb modelocked laser was initially motivated by the dual frequency VECSEL [36], where the VECSEL is operated in continuous wave $(\mathrm{cw})$. A recent study of noise correlation in such dual-frequency VECSELs [37] could be interesting for the "locked" regime of operation where both beams have the same pulse repetition rate. However, for dual comb spectroscopy applications we need two different pulse repetition rates and our focus in this paper is with regards to the origin of the decoupled timing jitter noise between the two output beams with different pulse repetition rates. We test our observation for two lasers, an optically pumped MIXSEL and Nd:YAG laser, using the same pump laser and the same SESAM for modelocking. These lasers operate in different regimes of noise properties [35,38,39] with strong relaxation oscillations, long upper state lifetimes and small gain cross sections in the latter case, but show very similar behavior with regards to the uncorrelated timing jitter.

\subsubsection{Phase noise of the dual-comb MIXSEL}

For the dual-comb MIXSEL (Fig. 4.2) we have a simple straight linear cavity defined by the MIXSEL chip and the output coupler (OC) as the two end mirrors. In this case it is straightforward to apply the concept of polarization-duplexing with a $\mathrm{CaCO}_{3}$ birefringent crystal inside the laser cavity, splitting the one cavity beam into two spatially separated and orthogonally polarized beams with slightly different pulse repetition frequencies of $1.895 \mathrm{GHz}$ and $1.890 \mathrm{GHz}$ [19]. The center wavelength of the two beams also slightly differs with $966.11 \mathrm{~nm}$ and $966.01 \mathrm{~nm}$, but 
their spectra are still well overlapping within the full width at half maximum of $0.25 \mathrm{~nm}$ and $0.23 \mathrm{~nm}$ for the s- and the p-polarized beam, respectively. The pulse duration for the s-polarized beam is $13.5 \mathrm{ps}$ and for the p-polarized beam $19.1 \mathrm{ps}$ and the average output power of each beam is $\approx 70 \mathrm{~mW}$. These modelocking parameters slightly vary for the different configurations tested within this paper. More details on the cavity and the modelocking performance can be found in [19].

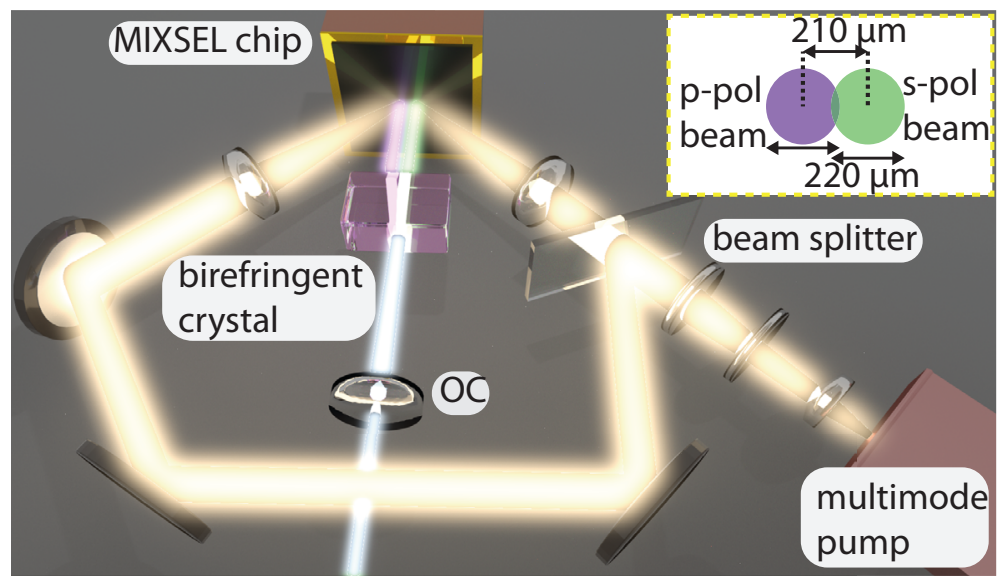

Figure 4.2: Dual-comb MIXSEL setup: the linear straight laser cavity is defined by the MIXSEL chip and output coupler (OC). An intracavity birefringent crystal is introduced to generate two beams (Fig. 4.1). The MIXSEL is pumped with a multimode semiconductor diode array which is split with a 50:50 beam splitter such that both cavity modes on the MIXSEL chip can be pumped under an angle of $45^{\circ}$. Inset: the two cavity modes on the MIXSEL chip with a diameter of $220 \mu \mathrm{m}$, separated by $210 \mu \mathrm{m}$ with a small overlap.

The phase noise of the s- and p-polarized beam is measured in freerunning and stabilized operation with a commercial signal source analyzer (SSA) (Agilent E5052B). The two collinear output beams are separated with a polarizing beam splitter (PBS) (Fig. 4.3), and each beam is independently fiber coupled and either the s-or the p-polarized beam is detected by a highly linear photodiode (PD1) (HLPD, Discovery Semiconductors Inc. DSC30S [40]). For the stabilization of the pulse repetition 
frequency, again part of either the s- or p-polarized beam is detected with a photodetector (PD2) (Thorlabs, DET01CFC) and then mixed in a double balanced mixer with a signal from a low noise electronic reference. The resulting phase error signal is then filtered with a custom designed proportional-integral loop filter [41] and the feedback of this phase-locked loop (PLL 1) is sent to the piezo-controlled output coupler to adjust the dual-comb laser cavity length. The PLL is adapted from previous pulse repetition frequency stabilizations of a MIXSEL [35].

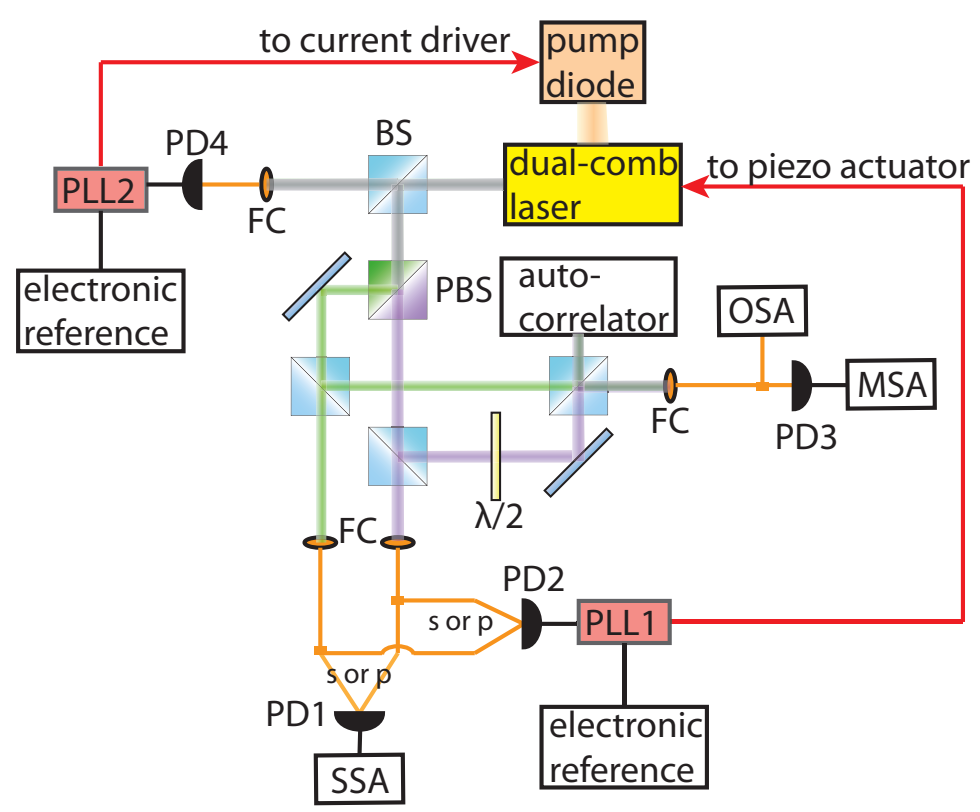

Figure 4.3: Laser characterization: each beam is characterized with a second harmonic autocorrelation, an optical spectrum analyzer (OSA), a microwave spectrum analyzer (MSA) and a signal source analyzer (SSA, Agilent E5052B). Two feedback loops can be applied. The first phase-locked loop (PLL1) gives a feedback of the error signal of one of the pulse repetition rates on the piezo-controlled output coupler to adjust the cavity length (i.e. for either the $\mathrm{s}$ or $\mathrm{p}$ polarized beam). The second phase-locked loop (PLL2) detects the interference of the two beams and gives a feedback of the error signal of the difference in pulse repetition rate on the current driver of the multimode pump; BS: beam splitter, PBS: polarizing beam splitter, FC: fiber coupling, PD: photodetector, $\lambda / 2$ : lambda half-wave plate 
The phase noise measurement with the SSA shows a noise reduction of over $100 \mathrm{~dB}$ for the s-polarized beam (Fig. 4(a)) from free-running operation (turquoise line) to the case of actively stabilizing the error signal of the s-polarized beam (green line). Turning on the active stabilization of the p-polarized beam and at the same time measuring the phase noise of the spolarized beam (blue line), shows that the stabilization of the p-polarized beam has in comparison no significant influence on the s-polarized beam. The same applies for the phase noise measurement of the p-polarized beam (Fig. 4.4(b)).
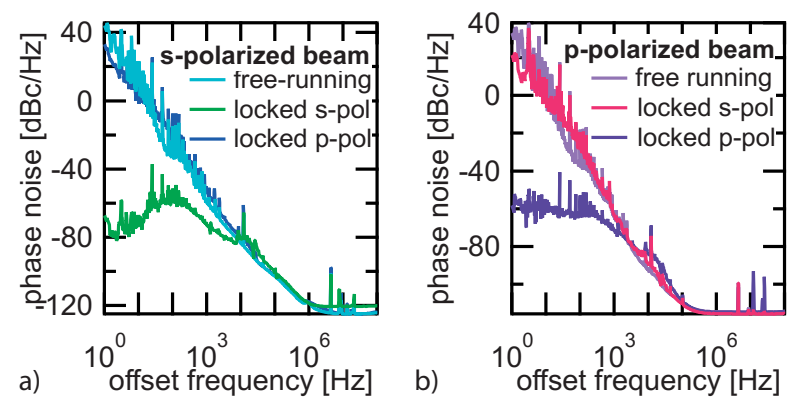

Figure 4.4: Dual-comb MIXSEL: power spectral densities of the measured timing phase noise for the arrival time of the pulses in each frequency comb [42] using a commercial signal source analyzer (SSA - Agilent E5052B) for the (a) s-polarized beam and for the (b) p-polarized beam, both in free-running operation, and with a feedback given on the error of the s-polarized beam (locked s-pol) or on the error of the p-polarized beam (locked p-pol). The vertical axis shows phase noise in units of $\mathrm{dBc}$ per hertz bandwidth: decibels relative to carrier i.e. $\mathrm{dB}$ below pulse repetition rate peak signal. The phase noise of both beams is uncorrelated. The integrated timing jitter over the whole measurement span from $1 \mathrm{~Hz}$ to $100 \mathrm{MHz}$ is in case of the s-polarized beam reduced by more than 3 orders of magnitude from $16.5 \mathrm{~ns}$ in free-running operation to $4.3 \mathrm{ps}$ with active stabilization of the spolarized beam. Same applies for the p-polarized beam with a reduction from $8 \mathrm{~ns}$ free-running to $4.8 \mathrm{ps}$ with active feedback on the p-polarized beam.

This uncorrelated timing jitter for the two frequency combs (Fig. 4.4) has initially been a surprise, since both beams share the same cavity and the feedback moves the output coupler for both beams. This implicates that the feedback of the stabilization is not only compensating mechanical vibrations of the cavity but accounts also for a noise source that is not 
common for both beams. We wanted to explore the most likely sources for these decoupled noise such as time delay on the saturable absorber [43] and pump laser noise.

We used a low-coherent multi-mode diode array laser to pump the MIXSEL. The short nanosecond upper-state lifetime of the semiconductor gain-material could potentially make the dual-comb MIXSEL more susceptible to the pump laser noise because the two cavity beams on the MIXSEL chip were both pumped under an angle of 45 degrees by splitting the pump beam with a 50:50 beam splitter (Fig. 4.2). Therefore, there is a spatial and temporal difference for the pump at the two spots on the gain. To explore this further we build a second dual comb laser using a Nd:YAG gain crystal with an upper state lifetime of $230 \mu$ s. The results with this laser are explained in more details in the next section.

\subsubsection{Nd:YAG dual-comb}

With a dual-comb Nd:YAG laser (Fig. 4.5) we need to separate the gain and absorber in two cavity elements which allows us to pump the gain for both combs fully overlapping in the gain. In this case both combs are pumped under identical conditions and the two beams are only separated on the SESAM. This will decouple the two most probable timing jitter noise sources. In addition the much longer upper state lifetime of the Nd:YAG laser (230 $\mu$ s versus nanoseconds) would reduce any possible pump noise induced timing jitter observed in the MIXSEL. The birefringent crystal is inserted in front of the SESAM (Fig. 4.5), such that the two cavity beams are spatially separated on the saturable absorber alone and perfectly overlap in the gain. With this configuration, the same geometry for the pump beam can be used to pump both beams simultaneously (Fig. 4.5(inset)). The diameter of the pump beam is chosen slightly smaller $(226 \mu \mathrm{m})$ than the diameter of the two cavity beams $(236 \mu \mathrm{m})$ to insure a good TEM00 beam profile. The M2 value is measured to be 1.1 or smaller for both beams in $x$ - and y-direction (Fig. 4.6 (a) and (b)). We used the same pump laser as before with the MIXSEL and a $1 \%$ output coupler. The modelocking performance of both beams is nearly identical in terms of beam profile, optical spectrum and pulse duration (Fig. 4.6). The average output power is $400 \mathrm{~mW}$ and $530 \mathrm{~mW}$ for the s- and p-polarized beam, 
respectively.

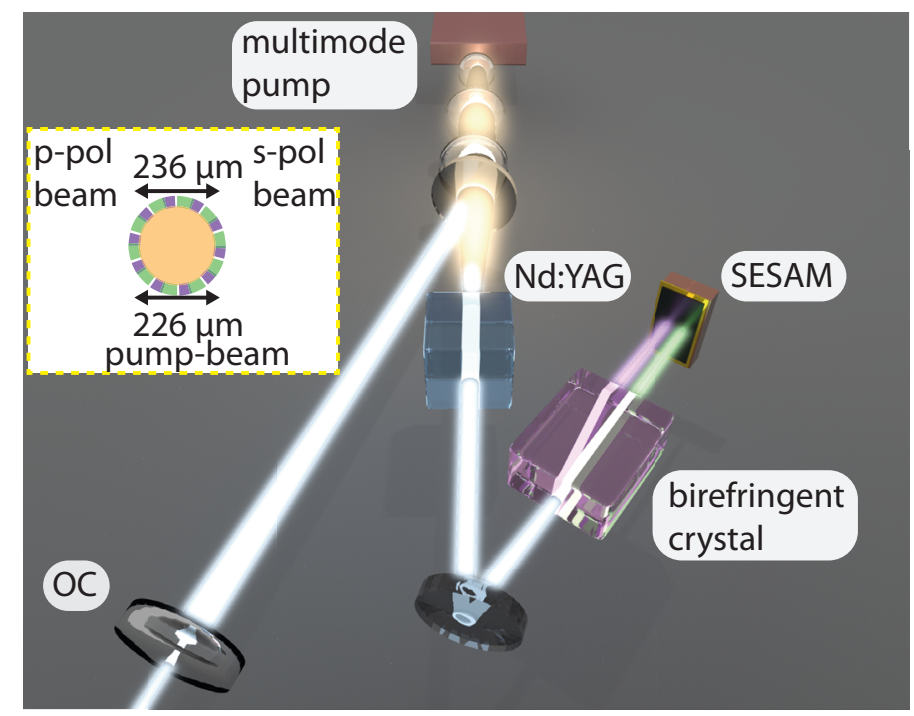

Figure 4.5: Dual-comb Nd:YAG laser setup: the cavity comprises (from left to right) an output coupler (OC), a flat dichroic folding mirror, the Nd:YAG crystal, a curved folding mirror to focus on the SESAM, the birefringent $\mathrm{CaCO} 3$ crystal and the SESAM. The pump beam is focused through the dichroic folding mirror into the Nd:YAG crystal. Inset: The s- and p-polarized beams are perfectly overlapping in the Nd:YAG crystal with a beam diameter of $236 \mu \mathrm{m}$ and a slightly smaller pump diameter of $226 \mu \mathrm{m}$.

The dual comb performance is similar as observed before with the MIXSEL [19]. We measured the microwave spectrum of the optical interference signal of the two combs on a fast photodetector (PD3) (NewFocus Model 1414) (Fig. 4.3). Optical interference is achieved when we turn the polarization of the p-polarized beam by 90 degrees with a lambda half-wave plate $(\lambda / 2)$ after passing the first beam splitter (BS) and then recombine with the s-polarized beam. The output signal of PD3 is shown on a microwave spectrum analyzer (MSA) between DC and the pulse repetition frequencies in a span of $1.2 \mathrm{GHz}$ and a resolution bandwidth (RBW) of $10 \mathrm{kHz}$ (Fig. 4.7(a)). A decreased span of $10 \mathrm{MHz}$ in a RBW of $3 \mathrm{kHz}$ (Fig. 4.7(b)) shows that the difference in pulse repetition frequen- 

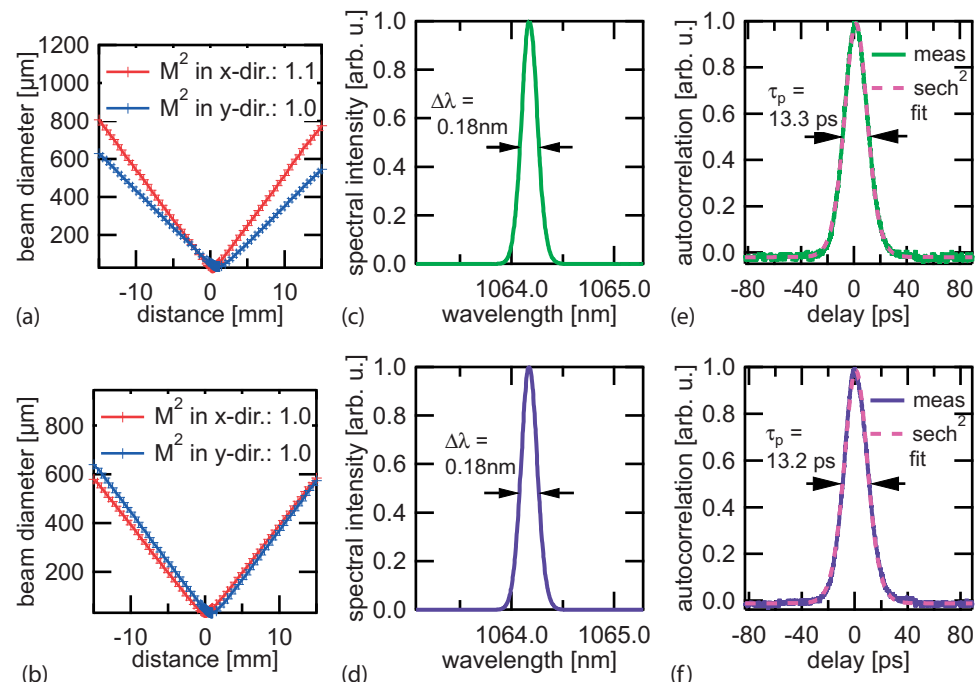

(d)

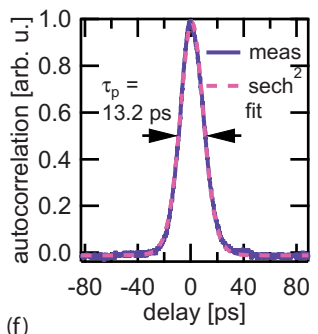

Figure 4.6: Dual-comb Nd:YAG laser: Beam profile M2 measurements of the (a) spolarized beam and of the (b) p-polarized beam. Optical spectrum of the (c) spolarized beam and of the (d) p-polarized beam, both centered at $1064.5 \mathrm{~nm}$. Second harmonic autocorrelation of the (e) s-polarized beam and of the (f) p-polarized beam revealing a pulse duration of around $13 \mathrm{ps}$.

cies $\left(\Delta f_{\text {rep }}\right)$, due to the different optical path-lengths in the birefringent crystal, is around $2.35 \mathrm{MHz}$. The difference frequency $\Delta f_{\text {rep }}$ appears also as additional side-peaks around DC and the pulse repetition frequencies. Very interesting for applications, for example for dual-comb spectroscopy, is the comb structure $\left(\mathrm{comb}_{1}\right)$ in between $\mathrm{DC}$ and the pulse repetition rates (Fig. 4.7(a)). In a reduced span of $200 \mathrm{MHz}$ and a $\mathrm{RBW}$ of $10 \mathrm{kHz}$, the individual comb lines are visible, which are spaced by $\Delta f_{\text {rep }}$ (Fig. 4.7(c)). This comb is only visible, if we observe optical interference of both beams on PD 3. This comb represents a direct link between the optical terahertz frequencies and the electronically accessible microwave regime. 

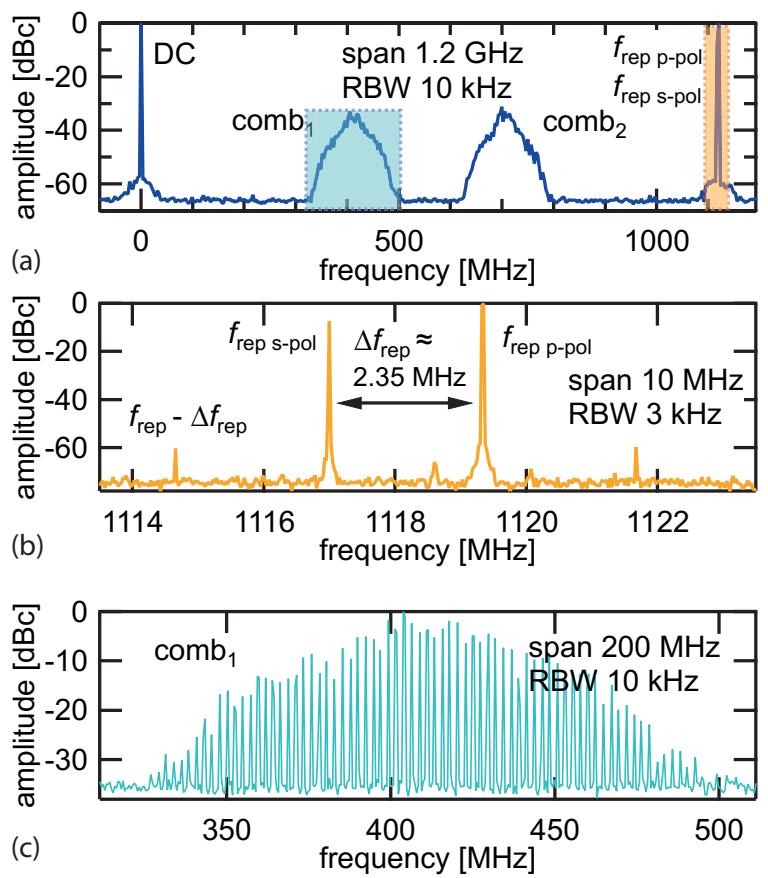

Figure 4.7: Dual-comb Nd:YAG laser with a 3-mm-thick birefringent plate (Fig. 4.5): (a) microwave spectrum analyzer (MSA) signal from DC to $1.2 \mathrm{GHz}$ with a resolution bandwidth (RBW) of $10 \mathrm{kHz}$. (b) Zoom-in around the pulse repetition frequencies with a span of $10 \mathrm{MHz}$ and a RBW of $3 \mathrm{kHz}$. (c) Zoom-in around the first comb (comb 1$)$ with a span of $200 \mathrm{MHz}$ and a RBW of $10 \mathrm{kHz}$.

\subsubsection{Noise correlation study}

We use the same timing jitter characterization configuration (Fig. 4.3) and observe the same uncorrelated timing jitter between the two beams (Fig. 4.8) as before with the dual-comb MIXSEL (Fig. 4.4). Therefore, we can conclude that the pump noise is not the source for the uncorrelated timing jitter of the two beams.

We used the dual-comb lasers for further parameter studies that are summarized in Table 4.1. The Nd:YAG laser allows for studies with beam overlap on the SESAM independent of the overlap on the gain. In configu- 

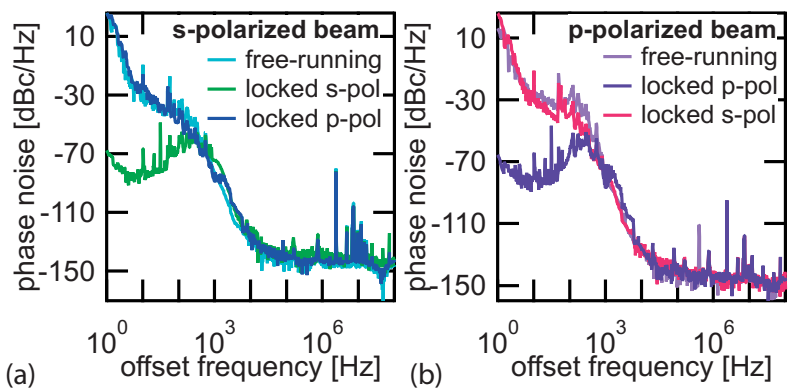

Figure 4.8: Dual-comb Nd:YAG laser: power spectral densities of the measured timing phase noise for the arrival time of the pulses in each frequency comb [42] using a commercial signal source analyzer (SSA - Agilent E5052B) for the (a) spolarized beam and for the (b) p-polarized beam, both in free-running operation, and with a feedback given on the error of the s-polarized beam (locked s-pol) or on the error of the p-polarized beam (locked p-pol). The phase noise of both beams is uncorrelated as before with the dual-comb MIXSEL (Fig. 4.4). The integrated timing jitter over the whole measurement span from $1 \mathrm{~Hz}$ to $100 \mathrm{MHz}$ is in case of the s-polarized beam reduced again by nearly 3 orders of magnitude from $2 \mathrm{~ns}$ in free-running operation to $6.4 \mathrm{ps}$ with active stabilization of the s-polarized beam. Same applies for the p-polarized beam with a reduction from 856 ps free-running to $5.8 \mathrm{ps}$ with active feedback on the p-polarized beam.

Table 4.1: Overview of the different laser configurations used in this paper

\begin{tabular}{|c|c|c|c|c|c|c|c|}
\hline configuration & 1 & 2 & 3 & 4 & 5 & 6 & 7 \\
\hline laser & MIXSEL & Nd:YAG & Nd:YAG & Nd:YAG & MIXSEL & MIXSEL & MIXSEL \\
\hline $\begin{array}{l}\text { same pump } \\
\text { configuration }\end{array}$ & $x$ & $\sqrt{ }$ & $\checkmark$ & $\checkmark$ & $x$ & $x$ & $x$ \\
\hline $\begin{array}{l}\text { overlap on } \\
\text { gain }\end{array}$ & partly & full & full & full & partly & partly & partly \\
\hline $\begin{array}{l}\text { overlap on } \\
\text { saturable } \\
\text { absorber }\end{array}$ & partly & $x$ & partly & $x$ & partly & partly & partly \\
\hline $\begin{array}{l}\text { feedback on } \\
f_{\text {rep }, 1} \text { via piezo }\end{array}$ & $\checkmark$ & $\sqrt{ }$ & $x$ & $x$ & $\checkmark$ & $\checkmark$ & $x$ \\
\hline$\Delta f_{\text {rep }}$ & $5 \mathrm{MHz}$ & $2.5 \mathrm{MHz}$ & $0 \mathrm{kHz}$ & $88 \mathrm{kHz}$ & $0 \mathrm{kHz}$ & $5 \mathrm{MHz}$ & $5 \mathrm{MHz}$ \\
\hline $\begin{array}{l}\text { feedback on } \\
\Delta f_{\text {rep via pump }} \\
\text { current }\end{array}$ & $x$ & $x$ & $x$ & $x$ & $x$ & $\checkmark$ & $\checkmark$ \\
\hline $\begin{array}{l}\text { passive locking } \\
\text { of pulse } \\
\text { repetition rate }\end{array}$ & $x$ & $x$ & $\checkmark$ & $x$ & $\checkmark$ & $x$ & $x$ \\
\hline $\begin{array}{l}\text { stabilization of } \\
f_{\text {rep }, 1} \text { and } f_{\text {rep }, 2}\end{array}$ & $x$ & $x$ & $x$ & $x$ & $\checkmark$ & $\checkmark$ & $x$ \\
\hline
\end{tabular}


ration number 1 and $2, \Delta f_{\text {rep }}$ between the two perpendicularly polarized output combs is in the megahertz range. In a configuration number 3 (Table 4.1) with the dual-comb Nd:YAG laser we have inserted a second intracavity birefringent crystal with the same thickness of $1 \mathrm{~mm}$ but with the optical axis rotated by 90 degrees such that both beams have approximately the same optical path length inside the cavity (Fig. 4.9(a)). We still maintain a small spatial overlap of the two beams on the SESAM (Fig. 4.9(b)) but now in addition we also allow for a temporal overlap of the two pulses. The microwave spectrum of the two superimposed beams is measured again in the same polarization (Fig. 4.10(a)). Between DC and the pulse repetition rate there is in this case only a single strong signal left instead of the previous microwave comb structure (comb 1 in Fig. 4.7). The repetition rate of the two beams is exactly the same $\left(\Delta f_{\text {rep }}=0 \mathrm{~Hz}\right)$, because passive locking of the pulse repetition rate occurs between the two beams with the additional temporal overlap. Therefore, the only difference in their frequency combs is the carrier envelope offset frequencies, and the measured signal is the relative carrier envelope offset $\Delta f_{\mathrm{CEO}}$ with a signal to noise ratio of more than $40 \mathrm{~dB}$ (Fig. 4.10(b)).

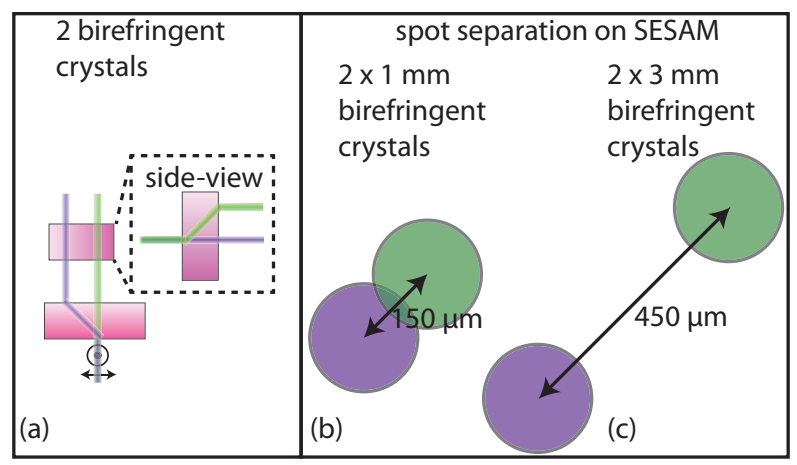

Figure 4.9: (a) Insertion of a second birefringent crystal of same thickness but with the optical axis rotated by $90^{\circ}$, such that both beams have approximately the same optical path length. (b) In configuration number 3 (Table 4.1), the beams overlap slightly on the SESAM with a distance between the centers of the two beams of $\approx 150 \mu \mathrm{m}$. The beam diameter is for both beams $\approx 180 \mu \mathrm{m}$. (c) In configuration number 4 (Table 4.1) the distance between the beams on the SESAM is increased to $\approx 450 \mu \mathrm{m}$, such that there is no overlap between the beams. 


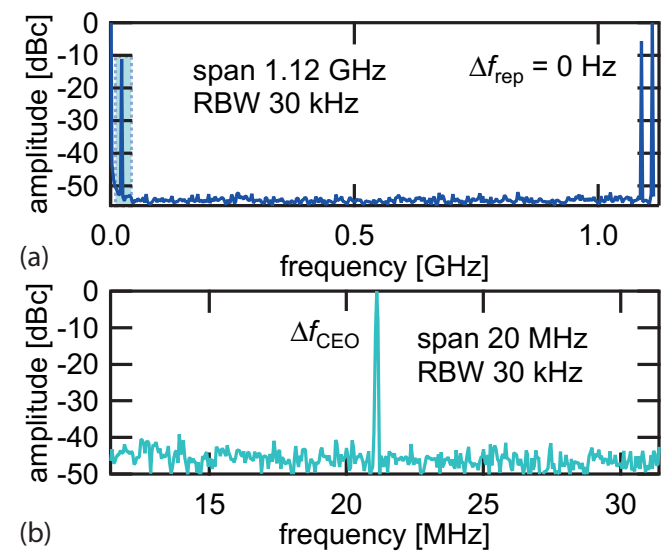

Figure 4.10: Dual-comb Nd:YAG laser with two 1-mm-thick birefringent crystals (Fig. 4.9(a)) to sufficiently reduce the different optical cavity length of the two beams to passively lock the two pulse repetition rates: (a) Microwave spectrum analyzer (MSA) signal from DC to $1.12 \mathrm{GHz}$. Both beams have exactly the same pulse repetition frequency due to passive locking of two overlapping pulses in the saturable absorber (Fig. 4.9(b)). Therefore, the only difference in frequency between the two beams is the carrier envelope offset (CEO) frequency. (b) Instead of a comb structure (comb $b_{1}$ in Fig. 4.7) we observe only one single strong frequency signal which is the relative CEO frequency $\left(\Delta f_{\mathrm{CEO}}\right)$ between the two combs.

To further confirm our conclusions from configuration number 3 we studied configuration number 4 for which we increased the beam separation to obtain no pulse overlap on the SESAM (Fig. 4.9(c)). In this case we used two birefringent crystals of $3 \mathrm{~mm}$ thickness. Sure enough we did not observe the passive locking of the pulse repetition rate (Fig. 4.11(a)). A zoom-in on the signal between DC and the repetition rates (Fig. 4.11(b)) reveals again a comb structure with a comb-line spacing of $88 \mathrm{kHz}$, which is the difference in pulse repetition rate.

In configuration number 5 we measure the timing phase noise of both beams with active stabilization under the condition of passive locking of the pulse repetition rate (i.e. with two intracavity birefringent crystals (Fig. 4.9(a)). With the Nd:YAG laser, the SESAM modelocking is, however, at its stability limit due to the increased loss of the two birefringent plates at gigahertz pulse repetition rates. We therefore continued with the MIXSEL 


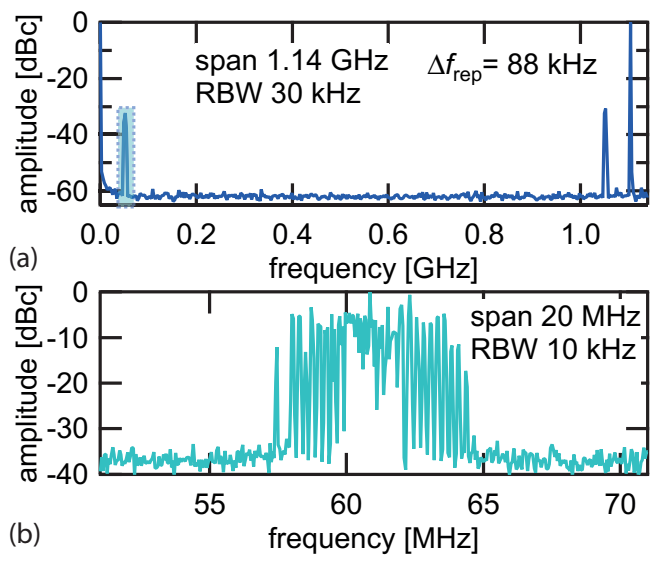

Figure 4.11: Dual-comb Nd:YAG laser with two 3-mm-thick birefringent crystals (Fig. 4.9(c)) to obtain no spatial beam overlap on the SESAM but still with significant temporal overlap: (a) microwave spectrum analyzer (MSA) signal from DC to $1.14 \mathrm{GHz}$. The difference in pulse repetition frequency $\left(\Delta f_{\text {rep }}\right)$ is $88 \mathrm{kHz}$ since no passive pulse repetition rate locking occurs because the beams do not overlap on the saturable absorber. (b) Zoom-in around the first comb with a span of $20 \mathrm{MHz}$ and a RBW of $10 \mathrm{kHz}$. The comb-line-spacing is reduced to $88 \mathrm{kHz}$, because it is set by $\Delta f_{\text {rep. }}$.

which does not suffer from Q-switching instabilities. The phase noise measurement (Fig. 4.12(a) and 4.12(b)) shows, that the s-polarized and the p-polarized beam are stabilized simultaneously, independent of the specific beam used for the feedback control. Thus with partial pulse overlap on the MIXSEL chip the timing phase noise becomes correlated. They are both stabilized simultaneously, however the pulse repetition frequency is also the same for both beams. Typically however, for most applications two different but still stabilized pulse repetition rates are required. For a strongly saturated absorber only the leading edge of the pulse experiences absorption which introduces an effective pulse delay (Fig. 4.13(a)) [43]. For our laser the incident pulse fluence on the saturable absorber is more then 10-times larger than the saturation fluence of the absorber. This means that the pulse is shifted back in time with each roundtrip. The estimated pulse delay is in the order of $10 \mathrm{fs}$ for a pulse duration of around 13 ps (Eq. (9) in [43]). This delay is not the same for both pulse 

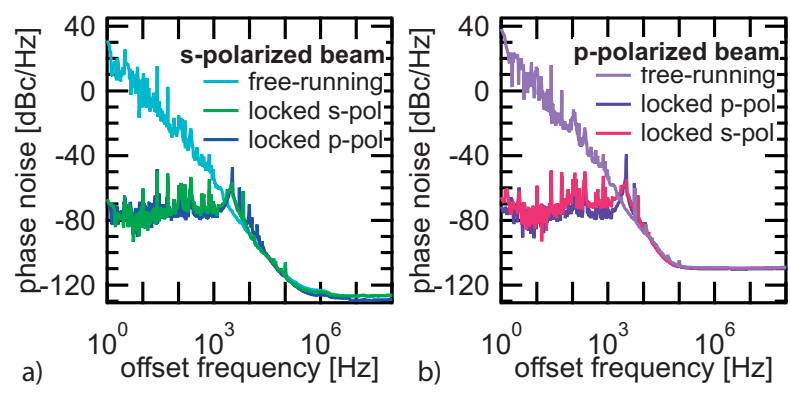

Figure 4.12: Timing phase noise of a dual-comb MIXSEL with two intracavity 1-mmthick birefringent crystals (Fig. 4.9(a)) with partial pulse overlap on the MIXSEL chip: (a) s-polarized beam and (b) p-polarized beam, both in free-running operation and with a feedback given on the error of the s-polarized beam (locked s-pol) or on the error of the p-polarized beam (locked p-pol). The phase noise of both beams is correlated.

trains, because it depends on the energy in each pulse and of course also on the saturable absorber. Small inhomogeneity in SESAM and MIXSEL will result in slightly different time delays and also explained the slightly different pulse performance in the two beams before [19]. However if the difference in roundtrip time is in the order of the saturation-induced pulse delay (e.g. for configuration number 3 in Tab. 1 a $\Delta f_{\text {rep }}=88 \mathrm{kHz}$ results in a difference in roundtrip time of $\Delta T_{\mathrm{rt}} \approx 70 \mathrm{fs}$ ) we could observe passive locking of the pulse repetition rate when the two beams also have a partial spatial overlap. If $\Delta f_{\text {rep }}$ is in the megahertz range, $\Delta T_{\mathrm{rt}}$ is in the picosecond range and therefore orders of magnitude larger than the saturation-induced pulse delay and no passive locking of the pulse repetition rate occurs.

For the dual-comb MIXSEL we therefore can only obtain stabilization of two different pulse repetition rates in the megahertz regime (i.e. with only one birefringent crystal as shown in Fig. 4.2) when we apply two feedback loops to stabilize the two uncorrelated noise sources. We can achieve this for a case with $\Delta f_{\text {rep }} \approx 5 \mathrm{MHz}$ when we use one feedback loop (PPL1 in Fig. 4.3) to adjust the cavity length based on the error signal of one beam and a second feedback loop (PPL2 in Fig. 4.3) to adjust the pump power on the error signal of the difference of the pulse repetition 


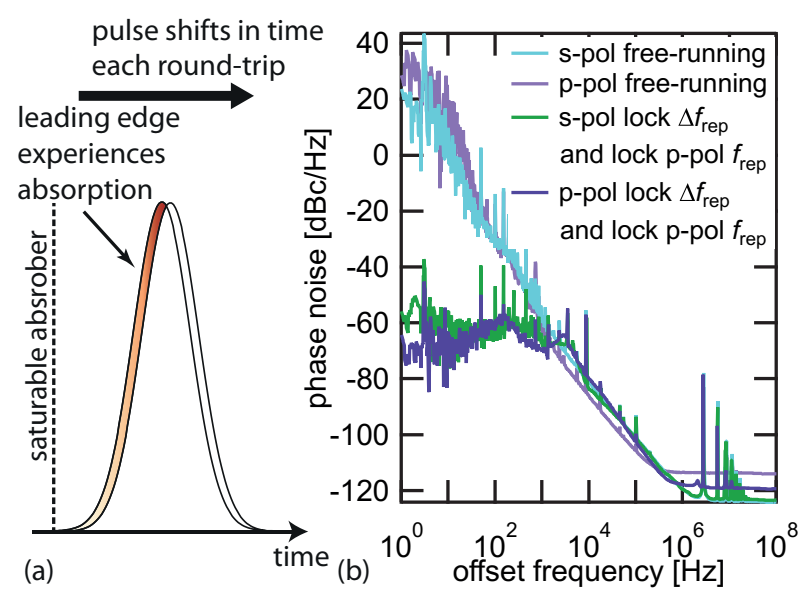

Figure 4.13: (a) Strongly saturated absorber introduces a pulse delay with each round-trip, because only the leading edge of the pulse experiences absorption since the absorber is already saturated for the trailing edge of the pulse. (b) Timing phase noise of the MIXSEL (Fig. 4.2) with only one birefringent crystal without pulse overlap in the MIXSEL chip. Both beams can only be stabilized simultaneously when two feedback loops are applied (Fig. 4.3).

rates (configuration number 6 in Table 4.1). In contrast to the microwave comb structure, which is a result of the optical interference of the two optical beams and can only be detected if both beams are superimposed in the same polarization, the detection of the difference in pulse repetition rate is independent of the polarization and can also be detected if the beams are cross-polarized. The difference in pulse repetition rate is then isolated using a low pass filter, and the signal is mixed with a low noise electronic reference signal. The measurement of the timing phase noise shows, that if both feedback loops are activated, both beams are stabilized for either using the s- or p-polarized beam for PPL1 (Fig. 4.13(b)). The feedback loop on the difference in pulse repetition frequencies accounts for the different pulse delays in the saturable absorber of the two beams. It is however not sufficient for the stabilization of the two beams to turn on only PLL2 (configuration number 7 in Table 4.1), because it only locks the phase noise of one beam to the noise of the other beam. Only if additionally PLL1 is turned on, both beams are stabilized. 


\subsubsection{Conclusion and outlook}

We have successfully demonstrated dual-comb lasers for both optically pumped semiconductor and Nd:YAG gain materials. An intracavity SESAM is used for passive modelocking and an intracavity birefringent filter is used to generate two collinear perpendicularly polarized output beams providing two frequency combs with two different pulse repetition rates from a single laser cavity (i.e. polarization-duplexed dual-comb laser).

We have successfully stabilized the pulse repetition rates for both gigahertz frequency combs. We explained the initially surprising observation that we need two feedback loops to stabilize the timing jitter for both beams when the laser is operated with significantly different pulse repetition rates. The different pulse delays for both beams, introduced by the strongly saturated absorber are responsible for the uncorrelated timing jitter of the two beams. We observed this uncorrelated noise for two different dual-comb lasers with very different gain materials such as semiconductor and Nd:YAG in terms of upper state lifetime and gain cross section. In both cases we used the same multi-mode diode laser array for pumping. We could demonstrate that in both cases the pump laser was not responsible for the uncorrelated noise of the pulse repetition rate in the two beams.

We could show that passive locking of the pulse repetition rate becomes possible with sufficient spatial and temporal pulse overlap in the absorber. In this case we only needed to apply one feedback loop to stabilize both frequency combs because the passive locking results in exactly the same pulse repetition rates in both beams. In this regime we have direct access to the difference in the frequency comb offset without any f-to-2f interferometric techniques [4].

The next step will be a more detailed analysis of the noise and possible stabilization schemes of the relative carrier envelope offset frequency. The MIXSEL structure used in this experiment supported only picosecond pulses. The recent progress in femtosecond MIXSELs [34] will support a much broader optical bandwidth centered around $1030 \mathrm{~nm}$. We are confident that in the near future gas-spectroscopy, for example on Acetylene, using a fully stabilized dual-comb MIXSEL will be presented, opening up 
this compact and inexpensive source for many applications in the field of frequency metrology, optical sensing and pump-probe experiments.

Acknowledgement The authors acknowledge support of the technology and clean room facility FIRST of ETH Zurich for advanced micro- and nanotechnology. This work was financed by the Swiss Confederation Program Nano-Tera.ch, which was scientifically evaluated by the Swiss National Science Foundation (SNSF).

\subsubsection{References and links}

1. D. Cotter, "Technique for highly stable active mode-locking," in Ultrafast Phenomena IV (Monterey, 1984), pp. 78-80.

2. M. J. W. Rodwell, D. M. Bloom, and K. J. Weingarten, "Subpicosecond laser timing stabilization," IEEE J. Quantum Electron. 25(4), 817-827 (1989).

3. A. Schlatter, B. Rudin, S. C. Zeller, R. Paschotta, G. J. Spühler, L. Krainer, N. Haverkamp, H. R. Telle, and U. Keller, "Nearly quantumnoise-limited timing jitter from miniature Er:Yb:glass lasers," Opt. Lett. 30(12), 1536-1538 (2005).

4. H. R. Telle, G. Steinmeyer, A. E. Dunlop, J. Stenger, D. H. Sutter, and U. Keller, "Carrier-envelope offset phase control: A novel concept for absolute optical frequency measurement and ultrashort pulse generation," Appl. Phys. B 69(4), 327-332 (1999).

5. D. J. Jones, S. A. Diddams, J. K. Ranka, A. Stentz, R. S. Windeler, J. L. Hall, and S. T. Cundiff, "Carrier-envelope phase control of femtosecond mode-locked lasers and direct optical frequency synthesis," Science 288(5466), 635-639 (2000).

6. A. Apolonski, A. Poppe, G. Tempea, C. Spielmann, T. Udem, R. Holzwarth, T. W. Hänsch, and F. Krausz, "Controlling the phase evolution of few-cycle light pulses," Phys. Rev. Lett. 85(4), 740-743 (2000).

7. V. Gerginov, C. E. Tanner, S. A. Diddams, A. Bartels, and L. Hollberg, "High-resolution spectroscopy with a femtosecond laser frequency 
comb," Opt. Lett. 30(13), 1734-1736 (2005).

8. J. Mandon, G. Guelachvili, and N. Picqué, "Fourier transform spectroscopy with a laser frequency comb," Nat. Photonics 3(2), 99-102 (2009).

9. T. Steinmetz, T. Wilken, C. Araujo-Hauck, R. Holzwarth, T. W. Hänsch, L. Pasquini, A. Manescau, S. D'Odorico, M. T. Murphy, T. Kentischer, W. Schmidt, and T. Udem, "Laser frequency combs for astronomical observations," Science 321(5894), 1335-1337 (2008).

10. D. Hillerkuss, R. Schmogrow, T. Schellinger, M. Jordan, M. Winter, G. Huber, T. Vallaitis, R. Bonk, P. Kleinow, F. Frey, M. Roeger, S. Koenig, A. Ludwig, A. Marculescu, J. Li, M. Hoh, M. Dreschmann, J. Meyer, S. Ben Ezra, N. Narkiss, B. Nebendahl, F. Parmigiani, P. Petropoulos, B. Resan, A. Oehler, K. Weingarten, T. Ellermeyer, J. Lutz, M. Moeller, M. Huebner, J. Becker, C. Koos, W. Freude, and J. Leuthold, " $26 \mathrm{Tbit} \mathrm{s}^{-1}$ line-rate super-channel transmission utilizing all-optical fast Fourier transform processing," Nat. Photonics 5(6), 364-371 (2011).

11. D. Hillerkuss, R. Schmogrow, M. Meyer, S. Wolf, M. Jordan, P. Kleinow, N. Lindenmann, P. C. Schindler, A. Melikyan, X. Yang, S. Ben-Ezra, B. Nebendahl, M. Dreschmann, J. Meyer, F. Parmigiani, P. Petropoulos, B. Resan, A. Oehler, K. Weingarten, L. Altenhain, T. Ellermeyer, M. Moeller, M. Huebner, J. Becker, C. Koos, W. Freude, and J. Leuthold, "Single-laser 32.4 Tbit/s Nyquist WDM transmission," J. Opt. Commun. Netw. 4, 715-723 (2012).

12. F. Keilmann, C. Gohle, and R. Holzwarth, "Time-domain midinfrared frequency-comb spectrometer," Opt. Lett. 29(13), 1542-1544 (2004).

13. A. Schliesser, M. Brehm, F. Keilmann, and D. van der Weide, "Frequency-comb infrared spectrometer for rapid, remote chemical sensing," Opt. Express 13(22), 9029-9038 (2005).

14. S. Schiller, "Spectrometry with frequency combs," Opt. Lett. 27(9), 766-768 (2002).

15. I. Coddington, W. C. Swann, and N. R. Newbury, "Coherent multi- 
heterodyne spectroscopy using stabilized optical frequency combs," Phys. Rev. Lett. 100(1), 013902 (2008).

16. B. Bernhardt, A. Ozawa, P. Jacquet, M. Jacquey, Y. Kobayashi, T. Udem, R. Holzwarth, G. Guelachvili, T. W. Hänsch, and N. Picqué, "Cavity-enhanced dual-comb spectroscopy," Nat. Photonics 4(1), 5557 (2010).

17. A. Bartels, R. Cerna, C. Kistner, A. Thoma, F. Hudert, C. Janke, and T. Dekorsy, "Ultrafast time-domain spectroscopy based on high-speed asynchronous optical sampling," Rev. Sci. Instrum. 78(3), 035107 (2007).

18. K. O. Hill, Y. Fujii, D. C. Johnson, and B. S. Kawasaki, "Photosensitivity in optical fiber waveguides: Application to reflection filter fabrication," Appl. Phys. Lett. 32(10), 647 (1978).

19. S. M. Link, A. Klenner, M. Mangold, C. A. Zaugg, M. Golling, B. W. Tilma, and U. Keller, "Dual-comb modelocked laser," Opt. Express 23(5), 5521-5531 (2015).

20. D. J. H. C. Maas, A.-R. Bellancourt, B. Rudin, M. Golling, H. J. Unold, T. Südmeyer, and U. Keller, "Vertical integration of ultrafast semiconductor lasers," Appl. Phys. B 88(4), 493-497 (2007).

21. U. Keller, K. J. Weingarten, F. X. Kärtner, D. Kopf, B. Braun, I. D. Jung, R. Fluck, C. Hönninger, N. Matuschek, and J. Aus der Au, "Semiconductor saturable absorber mirrors (SESAMs) for femtosecond to nanosecond pulse generation in solid-state lasers," IEEE J. Sel. Top. Quantum Electron. 2(3), 435-453 (1996).

22. U. Keller, "Recent developments in compact ultrafast lasers," Nature 424(6950), 831-838 (2003).

23. M. Kuznetsov, F. Hakimi, R. Sprague, and A. Mooradian, "Highpower ( $>0.5-\mathrm{W} \mathrm{CW}$ ) diode-pumped vertical-external-cavity surfaceemitting semiconductor lasers with circular TEM00 beams," IEEE Photonics Technol. Lett. 9(8), 1063-1065 (1997).

24. S. Calvez, J. E. Hastie, M. Guina, O. G. Okhotnikov, and M. D. Dawson, "Semiconductor disk lasers for the generation of visible and ultraviolet radiation," Laser Photonics Rev. 3(5), 407-434 (2009). 
25. B. Rösener, N. Schulz, M. Rattunde, C. Manz, K. Köhler, and J. Wagner, "High-power high-brightness operation of a 2.25- m (Al$\mathrm{GaIn}$ ) (AsSb)-based barrier-pumped vertical-external-cavity surfaceemitting laser," IEEE Photonics Technol. Lett. 20(7), 502-504 (2008).

26. D. J. M. Stothard, J.-M. Hopkins, D. Burns, and M. H. Dunn, "Stable, continuous-wave, intracavity, optical parametric oscillator pumped by a semiconductor disk laser (VECSEL)," Opt. Express 17(13), 10648-10658 (2009).

27. J. D. Berger, D. W. Anthon, A. Caprara, J. L. Chilla, S. V. Govorkov, A. Y. Lepert, W. Mefferd, Q.-Z. Shu, and L. Spinelli, "20 Watt CW TEM00 intracavity doubled optically pumped semiconductor laser at 532 nm," SPIE Proceedings 8242, 824206 (2012).

28. H. Kahle, R. Bek, M. Heldmaier, T. Schwarzbäck, M. Jetter, and P. Michler, "High optical output power in the UVA range of a frequency-doubled, strain-compensated AlGaInP-VECSEL," Appl. Phys. Express 7(9), 092705 (2014).

29. S. Hoogland, S. Dhanjal, A. C. Tropper, S. J. Roberts, R. Häring, R. Paschotta, F. Morier-Genoud, and U. Keller, "Passively mode-locked diode-pumped surface-emitting semiconductor laser," IEEE Photonics Technol. Lett. 12(9), 1135-1137 (2000).

30. U. Keller and A. C. Tropper, "Passively modelocked surface-emitting semiconductor lasers," Phys. Rep. 429(2), 67-120 (2006).

31. K. G. Wilcox, A. C. Tropper, H. E. Beere, D. A. Ritchie, B. Kunert, B. Heinen, and W. Stolz, " $4.35 \mathrm{~kW}$ peak power femtosecond pulse mode-locked VECSEL for supercontinuum generation," Opt. Express 21(2), 1599-1605 (2013).

32. P. Klopp, U. Griebner, M. Zorn, and M. Weyers, "Pulse repetition rate up to $92 \mathrm{GHz}$ or pulse duration shorter than $110 \mathrm{fs}$ from a modelocked semiconductor disk laser," Appl. Phys. Lett. 98(7), 071103 (2011).

33. C. A. Zaugg, A. Klenner, M. Mangold, A. S. Mayer, S. M. Link, F. Emaury, M. Golling, E. Gini, C. J. Saraceno, B. W. Tilma, and U. 
Keller, "Gigahertz self-referenceable frequency comb from a semiconductor disk laser," Opt. Express 22(13), 16445-16455 (2014).

34. M. Mangold, M. Golling, E. Gini, B. W. Tilma, and U. Keller, "Sub300-femtosecond operation from a MIXSEL," Opt. Express 23(17), 22043-22059 (2015).

35. M. Mangold, S. M. Link, A. Klenner, C. A. Zaugg, M. Golling, B. W. Tilma, and U. Keller, "Amplitude noise and timing jitter characterization of a high-power mode-locked integrated external-cavity surface emitting laser," IEEE Photonics J. 6(1), 1-9 (2014).

36. G. Baili, L. Morvan, M. Alouini, D. Dolfi, F. Bretenaker, I. Sagnes, and A. Garnache, "Experimental demonstration of a tunable dualfrequency semiconductor laser free of relaxation oscillations," Opt. Lett. 34(21), 3421-3423 (2009).

37. S. De, G. Baili, S. Bouchoule, M. Alouini, and F. Bretenaker, "Intensity- and phase-noise correlations in a dual-frequency verticalexternal-cavity surface-emitting laser operating at telecom wavelength," Phys. Rev. A 91(5), 053828 (2015).

38. U. Keller, T. H. Chiu, and J. F. Ferguson, "Self-starting and self-Qswitching dynamics of passively mode-locked Nd:YLF and Nd:YAG lasers," Opt. Lett. 18(3), 217-219 (1993).

39. C. Hönninger, R. Paschotta, F. Morier-Genoud, M. Moser, and U. Keller, "Q-switching stability limits of continuous-wave passive mode locking," J. Opt. Soc. Am. B 16(1), 46-56 (1999).

40. A. Joshi and S. Datta, “Dual InGaAs photodiodes having high phase linearity for precise timing applications," IEEE Photonics Technol. Lett. 21(19), 1360-1362 (2009).

41. V. J. Wittwer, R. van der Linden, B. W. Tilma, B. Resan, K. J. Weingarten, T. Sudmeyer, and U. Keller, "Sub- 60- fs timing jitter of a SESAM modelocked VECSEL," IEEE Photonics J. 5(1), 1400107 (2013).

42. U. Keller, K. D. Li, M. J. W. Rodwell, and D. M. Bloom, "Noise characterization of femtosecond fiber raman soliton lasers," IEEE J. Quantum Electron. 25(3), 280-288 (1989). 
43. R. Paschotta and U. Keller, "Passive mode locking with slow saturable absorbers," Appl. Phys. B 73(7), 653-662 (2001). 


\section{Chapter 5}

\section{Dual-comb spectroscopy}

OFCs are very versatile tools that have shown tremendous progress over the last 20 years and enabled and improved numerous applications. This chapter focuses on the application of spectroscopy, especially the detection of gas species and their concentration. If an OFC is sent through a gas, its equidistantly spaced comb of frequencies will be modulated according to the specific absorption lines of the gas (Fig. 5.1), assuming that the optical spectrum of the OFC overlaps with a spectral region where the gas exhibits absorption features. Reading out the frequencies of the

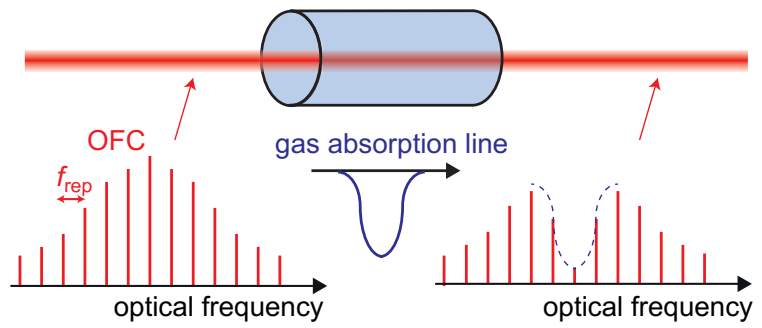

Figure 5.1: Gas spectroscopy principle with an optical frequency comb (OFC): The equidistantly spaced frequencies will be modulated according to the specific absorption lines of the gas under test. 
modulated OFC after the interaction with the gas gives the required information about the probed gas species and its concentration. The issue is that the optical frequencies of the OFC are in the range of several tens to hundreds of terahertz, which is faster than any available electronics could count. One established way of overcoming this issue is to use Fourier transform spectroscopy. This technique uses the interferences of one OFC with a delayed version of itself in an interferometric setup to down-convert the optical frequencies into a range where they can be directly measured with standard electronics. The drawback is that a long and variable delay line is needed in one of the arms of the spectrometer, which makes the detection setup rather bulky and slow, hindering a more wide-spread use of this technique for many applications.

\subsection{Dual-comb spectroscopy principle}

Dual-comb spectroscopy utilized the same down-conversion principle as Fourier transform spectroscopy but in a static manner, circumventing any moving part in the setup and therefore dramatically improving the acquisition speed and potentially also the setup size. Instead of delaying part of the beam and tuning the delay, a second OFC with a slightly different pulse repetition frequency $f_{\text {rep }}$ and therefore comb-line spacing is used to sample the first comb. The down-conversion in the frequency domain is described in Fig. 5.2. The beat notes between adjacent optical comb-lines (Fig. 5.2a) form a new frequency comb in the microwave regime (Fig. 5.2b), with the new comb-line spacing given by the difference in pulse repetition frequency $\Delta f_{\text {rep }}$ of the two OFCs. This microwave comb can be detected with standard electronics and represents a direct link between the optical frequencies and the microwave regime. The down-conversion coefficient $a$, also referred to as scaling factor [37], is give by

$$
a=\frac{\Delta f_{\text {rep }}}{f_{\text {rep }, 1}} .
$$

In the time-domain, the dual-comb approach is similar to a pumpprobe experiment, where one pulse train is sampled by the second pulse train (Fig. 5.3). The pulses of the two lasers are slowly walking over each other due to the slightly different pulse repetition rate (Fig. 5.3). If both 


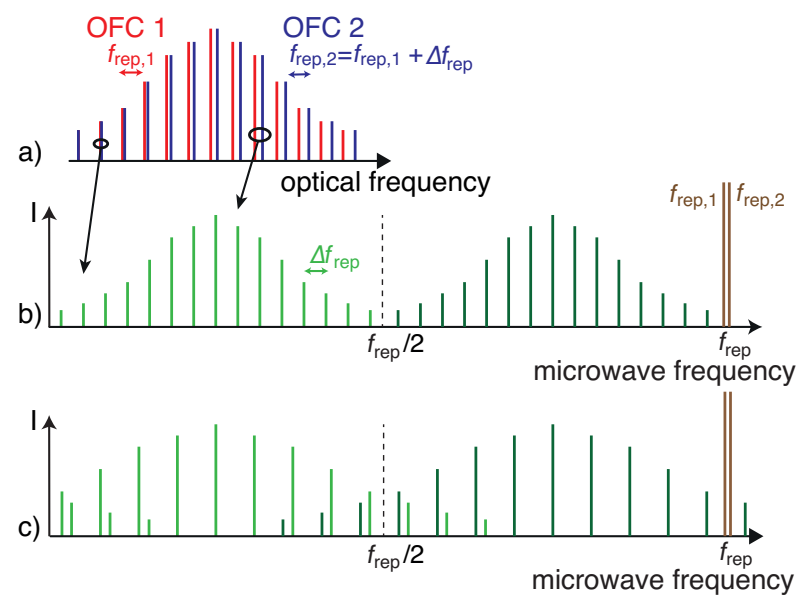

Figure 5.2: Dual-comb frequency down-conversion principle in the frequency domain. a) Two OFCs with slightly different comb-line spacing are superimposed on a detector. $b$ ) The beating between adjacent lines generates a frequency comb in the microwave regime. If the difference in pulse repetition rate $\Delta f_{\text {rep }}$ is chosen ideally, the microwave comb spans over half of the pulse repetition frequency $f_{\text {rep }} / 2$. c) If $\Delta f_{\text {rep }}$ is too large with respect to the optical bandwith, aliasing occurs and the different beat notes merge and become ambiguous.

pulses arrive at the same time on the detector, a burst is detected. This interferogram repeats every $1 / \Delta f_{\text {rep }}$, which corresponds to the acquisition time of a single interferogram. Therefore, the higher $\Delta f_{\text {rep }}$ is chosen, the faster the acquisition becomes. However, there is an upper limit for $\Delta f_{\text {rep }}$. If the difference in pulse repetition frequency is too large, and therefore the comb-line spacing of the microwave comb becomes to big, aliasing occurs and the beat notes start to merge and become ambiguous (Fig. 5.2c). The maximum value for $\Delta f_{\text {rep }}$ depends on the optical bandwidth $\Delta v$ of the OFCs [5]

$$
\Delta f_{\text {rep }} \leq \frac{f_{\text {rep }}^{2}}{2 \Delta v}
$$

In a dual-comb gas spectroscopy experiment, one OFC is sent through the gas cell and afterwards recombined with the second OFC on a detector. It is also possible to send both OFCs through the cell. This reduces the 


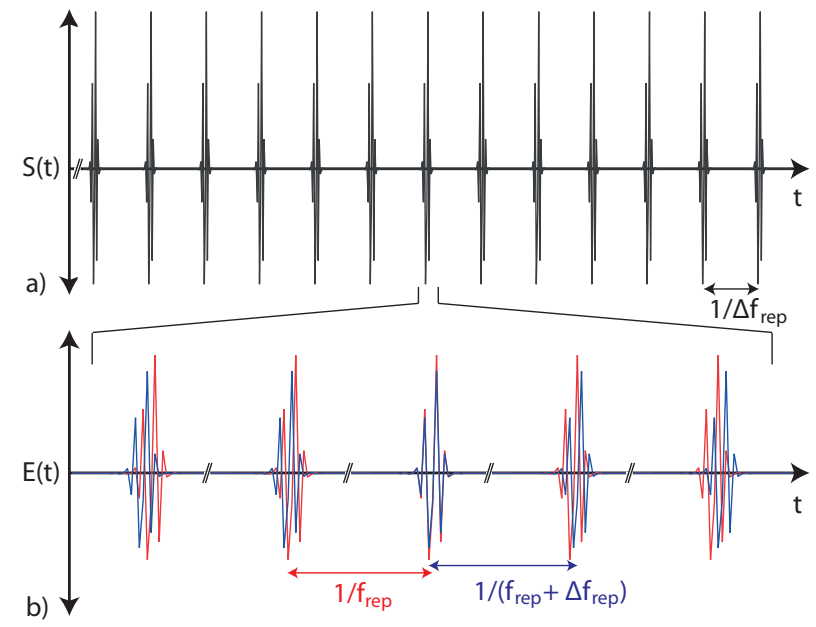

Figure 5.3: Dual-comb principle in the time domain. a) Every time a pulse of each OFC arrives simultaneously on the detector, a burst is visible. b) The pulses of the two OFCs are sampling each other by slowly walking over each other due to the slightly different pulse repetition rate.

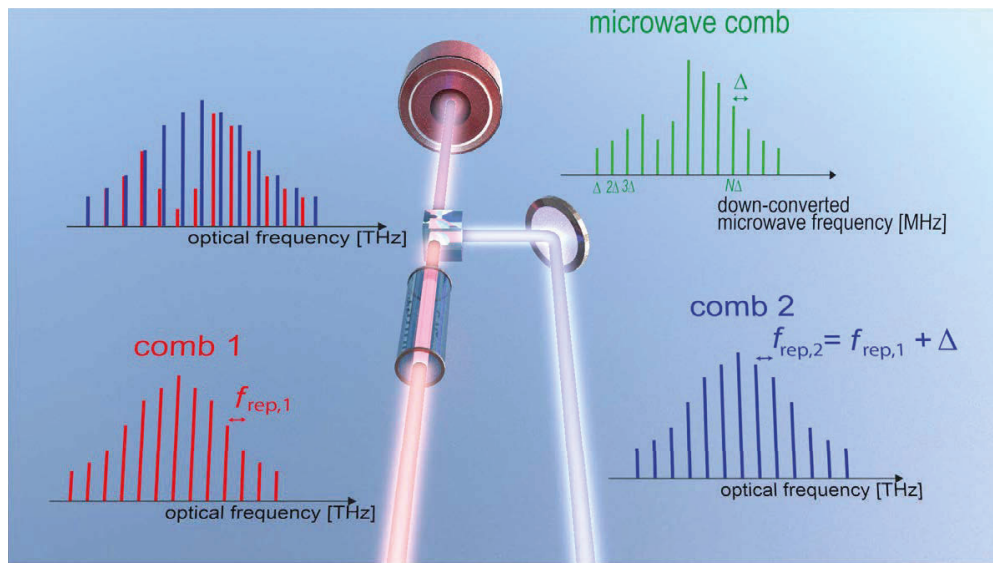

Figure 5.4: Dual-comb gas spectroscopy concept: one OFC (or both) is send through a gas cell and then both beams are superimposed on a detector. 
complexity of the setup and enhances the absorption but only allows for amplitude- and not for phase-sensitive measurements. The modulation of the OFC due to the absorption of the gas is transferred to the microwave comb (Fig. 5.4). Using the microwave comb one can calculate the absorption and with that the concentration of the gas. Due to the high acquisition speed and the non-invasive nature of the measurement using lasers, detection of flowing gases, e.g. in pipelines or exhausts of factories could be enabled.

\subsection{Laser sources for dual-comb spectroscopy}

The only drawback of dual-comb spectroscopy is the complexity and high price of its currently available laser sources. The required two OFCs need to be very stable and coherent with respect to each other, which usually demands for complex stabilization schemes. Most of the so far demonstrated dual-comb spectroscopy systems rely on two modelocked Ti:Sapphire or fiber lasers, that are broadened in a nonlinear fiber to generate an octavespanning supercontinuum. The supercontinuum is necessary to detect the CEO frequency in order to fully stabilize both lasers using self-referencing via f-to-2f interferometry [6]. Even though excellent results are achieved with these systems, their high complexity and cost limit them to the laboratory environment and prevent a wide-spread industrial use.

There is a strong ongoing effort to simplify the laser sources for dualcomb spectroscopy with several different approaches. Especially the use of quantum cascade lasers (QCLs) [38] and microresonators [39] found a lot of attraction. A brief overview of these two approaches is given in the following for comparison with our dual-comb MIXSEL method. Special consideration is given to the following key requirements for molecular spectroscopy:

- Power per line: A higher power per comb-line results in a better signal-to-noise ratio and therefore less averaging is needed, enabling faster measurement times.

- Optical comb-line spacing: The spacing of the optical comb-lines should be sufficiently narrow to resolve spectroscopic features (the 
line-spacing corresponds to the pulse repetition frequency in case of modelocked lasers).

- Optical linewidth: Also the optical linewidth of the individual comblines should be sufficiently narrow to resolve the spectroscopic features.

\subsubsection{QCL combs}

Due to the much shorter gain recovery time ( $\approx 300 \mathrm{fs})$ compared to the usual cavity roundtrip time $(\approx 60 \mathrm{ps})$ stable $\mathrm{cw}$ modelocking with QCLs at room-temperature is not practical [40]. Nonetheless the generation of frequency combs with QCLs is possible, however, it does not lead to short pulses in time. The QCL combs (Kerr combs) are generated via four-wavemixing (FWM). The optical transition of the QCL is an intraband transition in a semiconductor gain structure with a very high $\chi^{3}$ nonlinearity, allowing for broadband FWM. The locking of the phases of the generated frequencies can occur due to internal injection locking of newly generated modes to stronger existing modes in a master-slave configuration [40]. The injection locking requires a low GDD which is crucial and challenging. The bandwidth of locked frequencies $\Delta f_{\text {lock }}$ depends on the power of the master $P_{\mathrm{M}}$ and slave $P_{\mathrm{S}}$ mode as well as on the comb-line spacing $f_{\text {rep,QCL }}$ [40]

$$
\Delta f_{\text {lock }}=\frac{\eta f_{\text {rep }, \mathrm{QCL}}}{2 \pi^{2}} \sqrt{\frac{P_{\mathrm{M}}}{P_{\mathrm{S}}}} .
$$

For such QCL combs the phase difference between two successive modes is constant, however, in contrast to modelocked lasers, all phases are not the same but are related through a non-trivial relationship. Therefore, QCL combs do not produce a pulse train in the time domain, with the consequence that nonlinear frequency conversion and broadening can not be applied.

So far, it has been shown that micro heaters can be used to shift the comb offset and the repetition rate to overlap two combs, however, a feedback loop using micro heaters to fully stabilize the frequency comb has not yet been realized. The thermal control affects both, comb spacing and offset and it is not yet possible to control the two frequencies independently of each other [41]. 
The high repetition rate is usually in the range of $>7.5 \mathrm{GHz}$ [38]. Therefore, the optical comb spacing is wide which reduces the resolution for spectroscopy. A repetition rate of $7.5 \mathrm{GHz}$ corresponds to a device length of $\approx 6 \mathrm{~mm}$. Producing a QCL with a better suited repetition rate of 1 or $2 \mathrm{GHz}$ will be very challenging since it would require a structure of $\approx 45 \mathrm{~mm}$ length. In addition, Eq. 5.3 shows that there is a trade-off between short repetition rates and achievable phase-locked optical bandwidth.

The linewidth $\delta v$ of a laser depends on the Q-factor of the cavity and the optical center frequency $v_{0}$

$$
\delta v=\frac{v_{0}}{Q} .
$$

The $\mathrm{Q}$ factor is given by

$$
Q=v_{0} \frac{2 \pi}{f_{\text {rep }} l}
$$

with $l$ being the losses of the cavity.

Usually in a QCL nearly $70 \%$ of the light is coupled out through one $\mathrm{InP} /$ air facet. This leads to a very low finesse $(\approx 5)[42]$ and a $\mathrm{Q}$ factor in the order of $10^{3}$. The corresponding linewidth is with roughly $10 \mathrm{GHz}$ very broad. In addition, QCLs seem to suffer from flicker noise, which is yet to be solved [41].

Despite their current drawbacks and limitations QCLs are interesting sources for dual-comb spectroscopy since they cover wavelengths in the range of 7-10 $\mu \mathrm{m}$ [42] and with cryogenic cooling also 2-5 THz [43] with relatively high average output power. These spectral domains are interesting for spectroscopy and challenging to reach with other laser sources.

\subsubsection{Microresonators}

In a microresonator, a frequency comb can be generated in a nondegenerate FWM (or cascaded FWM) process [44] which leads to a phaselock between the different modes. This process is already possible with low intracavity power due to the high nonlinearity and the long interaction length of the microresonator. In contrast to QCLs, modelocking is possible with microresonators, however, the soliton formation and stabilization is a very delicate process [45]. 
Even more so than for QCLs, the optical comb spacing/repetition rate poses a problem for dual-comb spectroscopy. Most of the microresonator publications report repetition rates above $100 \mathrm{GHz}$. The reason for this is that the fabrication of long waveguides with large enough confinement and small enough optical losses is very challenging. The lowest repetition rate demonstrated so far with soliton-modelocked chip-based microresonators is $22 \mathrm{GHz}$ [39] and $12 \mathrm{GHz}$ for crystalline microresonators [46]. The large comb-line spacing leads to a coarse resolution, which poses a problem for dual-comb spectroscopy because it is not possible to resolve all the absorption details, as observed in the first dual-comb spectroscopy demonstration with microresonators [39].

The average output power is typically in the range of a few $\mu \mathrm{Ws}$ to $1 \mathrm{~mW}$. Therefore, the power per comb-line is low resulting in a very limited signal-to-noise ratio. On the other hand, the Q-factor of microresonators is very high $\left(>10^{8}\right)$ with a small corresponding optical linewidth at $1550 \mathrm{~nm}$ of roughly $1-10 \mathrm{MHz}$. In addition, a spectral bandwidth covering up to an octave has been presented with microresonators [44].

\subsubsection{Further OFC sources}

Besides these two laser technologies, there are also other approaches with the goal to simplify dual-comb spectroscopy sources such as a bidirectional fiber-ring laser [47] or frequency agile modulation of a cw laser with electro-optic modulators [48]. These methods have different drawbacks at this point in time but show a lot of potential and could evolve to interesting alternatives. However, the aim of this thesis is not to give an exhaustive review on all possible dual-comb sources.

\subsubsection{Dual-comb MIXSEL}

In contrast to QCLs and microresonators, the dual-comb MIXSEL works well with a pulse repetition rate below $2 \mathrm{GHz}$, as shown in chapter 3, enabling the detection of much narrower spectroscopic features. The cavity losses and the output coupling rate are small in a MIXSEL cavity, which leads to a high $Q$ factor in the range of $10^{7}-10^{8}$ and a corresponding optical linewidth below $10 \mathrm{MHz}$. As demonstrated in chapter 2, the noise proper- 
ties of the passively modelocked MIXSEL are excellent, with a comb-line spacing variation of $\approx 2.5 \times 10^{-4}$ integrated over $10 \mathrm{~ms}$ in free-running operation [32]. Due to the generation of short pulses in time in the modelocking process, external nonlinear difference frequency generation or broadening are easily applicable. In the dual-comb MIXSEL demonstration in chapter 3 , the average power per comb-line exceeds $1 \mathrm{~mW}$.

Since the specifications of the dual-comb MIXSEL fulfill nicely the requirements for dual-comb spectroscopy, this promising source needs to be tested in a gas spectroscopy experiment. The first gas spectroscopy demonstration of water vapor using a dual-comb MIXSEL is presented in the following Science publication [49].

\title{
5.3 Dual-comb spectroscopy of water vapor with a free- running semiconductor disk laser
}

\author{
S. M. Link, ${ }^{1 *}$ D. J. H. C. Maas, ${ }^{2}$ D. Waldburger, ${ }^{1}$ U. Keller ${ }^{1}$
}

${ }^{1}$ Department of Physics, Institute for Quantum Electronics, ETH Zürich, 8093 Zürich, Switzerland.

${ }^{2}$ ABB Switzerland Ltd., Corporate Research, Segelhofstrasse 1K, 5405 Baden-Daettwil, Switzerland.

Dual-comb spectroscopy offers the potential for high accuracy combined with fast data acquisition. Applications are often limited, however, by the complexity of optical comb systems. Here we present dual-comb spectroscopy of water vapor using a substantially simplified single-laser system. Very good spectroscopy measurements with fast sampling rates are achieved with a free-running dual-comb modelocked semiconductor disk laser. The absolute stability of the optical comb modes is characterized both for free-running operation and with simple microwave stabilization. This approach drastically reduces the complexity for dual-comb spectroscopy. Band-gap engineering to tune the center wavelength from the UV to the mid-IR could optimize frequency combs for specific gas targets, further enabling dual-comb spectroscopy for a wider range of industrial applications. 
A fully stabilized optical frequency comb (OFC) consists of equally spaced frequencies with a relative uncertainty as low as $10^{-19}$ (1) and can serve as a very precise ruler for optical frequency measurements. The combs can be generated by mode-locked lasers with sufficient peak power in femtosecond pulses for supercontinuum generation in a single pass through a fiber or waveguide (2-4); generation with cw lasers (5) requires sufficient average power in a narrow-frequency linewidth to couple into a high-Q microresonator for highly nonlinear parametric interactions. Many applications require frequency combs in the $100-\mathrm{MHz}$ to $10-\mathrm{GHz}$ regime, where equally spaced microresonator combs become more challenging (6). Although mode-locked laser technology has enabled frequency-spacing stabilization since the 1980 s $(7,8)$, stabilizing the frequency comb offset requires substantial spectral broadening (2). To date, stabilized GHz OFCs have had to rely on rather bulky and complex ultrafast diode-pumped solid-state (9), fiber (10), or Ti:sapphire lasers (11). In comparison, optically pumped semiconductor disk lasers such as the MIXSELs (modelocked integrated external-cavity surface-emitting lasers) (12) are often better suited for mass production and widespread applications, as they are based on a wafer-scale technology with reduced packaging requirements and potentially a higher level of integration.

Here we demonstrate dual-comb spectroscopy of water vapor with a single mode-locked laser cavity. The dual-comb mode-locked laser (13) generates two collinear, perpendicularly polarized OFCs with an adjustable frequency difference in comb spacing. A fast scan rate and the single-laser cavity approach support spectroscopic measurements even using the free-running laser without any additional external spectral broadening or stabilization. This is a potential paradigm shift in frequency metrology: to use a narrowband OFC, which is then tuned to the desired spectral range based on the specific target to be tested. This approach provides more power per comb line and a resulting higher signal-to-noise ratio (Fig. 5.5A). A dual-comb MIXSEL generates two OFCs from the same laser cavity and provides a potentially very attractive source for this approach. Tuning the OFC center wavelength from UV to mid-IR for different gas targets can be achieved with semiconductor band-gap engineering (14-19) (Fig. 5.5B). For the demonstration on water vapor, the dual-comb 

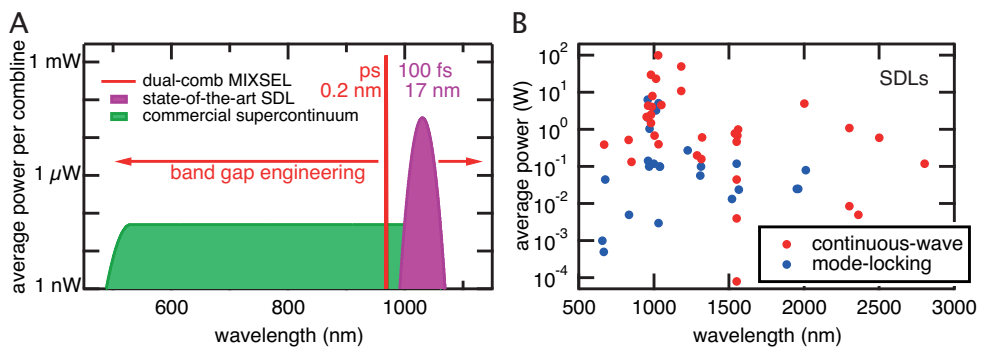

Figure 5.5: Current state-of-the-art performance in frequency comb characteristics. (A) A commercial OFC (e.g., Menlo FC1500-250-WG MVIS) can cover an octave of optical spectrum. The large bandwidth, however, strongly reduces the average power per comb line to about $50 \mathrm{nW}$. Optically pumped semiconductor disk lasers (SDLs) can provide more than $1 \mathrm{~mW}$ of average power per comb line. With pulse durations as short as $100 \mathrm{fs}$, their optical spectrum spans up to $17 \mathrm{~nm}$ at a center wavelength around $1 \mathrm{~mm}$ (41). The center wavelength of ultrafast SDLs can be shifted with semiconductor band-gap engineering. (B) Large spectral coverage of optically pumped SDLs. Overview of continuous-wave and passively mode-locked SDL results in terms of center operation wavelength and average output power on a logarithmic scale. The plotted results are summarized in recent review papers (14-19).

MIXSEL (Fig. 5.6A) was operated around a center wavelength of $968 \mathrm{~nm}$ with a pulse repetition rate of $\approx 1.7 \mathrm{GHz}$ and a frequency offset of $4 \mathrm{MHz}$ with an average power of $>60 \mathrm{~mW}$ and a pulse duration of about $18 \mathrm{ps}$ (Fig. 5.9).

Dual-comb spectroscopy is generally very attractive for molecular spectroscopy $(20,21)$, as well as asynchronous optical sampling (22), distance measurements (23), and fiber Bragg grating sensing (24). The technique often offers dramatic improvements in spectral resolution, sensitivity, and data-acquisition speed compared to traditional optical spectrometer techniques. With the dual-comb principle, a direct link between the optical frequencies and the microwave regime is established (Fig. 5.6B). This dualcomb approach is very attractive for applications such as spectroscopy. A key drawback of dual-comb spectroscopy is the requirement for two fully stabilized OFCs with slightly different comb spacing (Fig. 5.6B). For mode-locked lasers, this requires stabilization of both the pulse repetition frequency (i.e., the frequency comb spacing) and the carrier envelope off- 


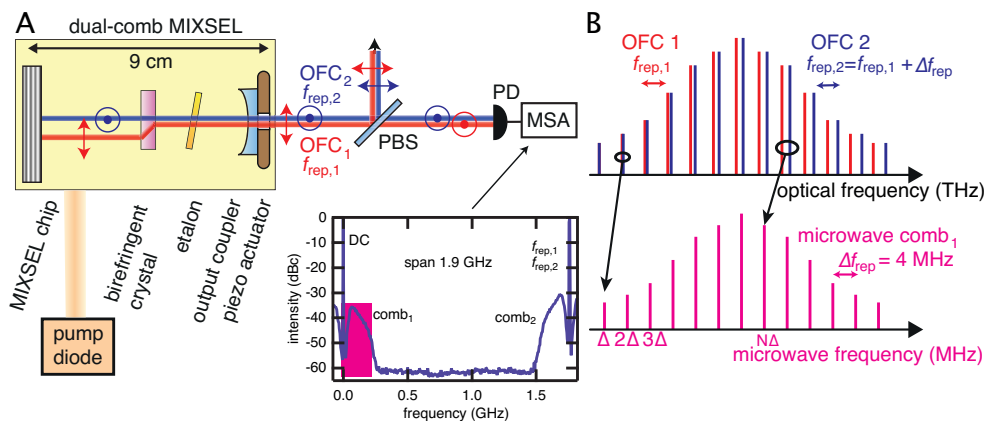

Figure 5.6: Dual-comb source and operating principle. (A) Dual-comb MIXSEL. The diode-pumped dual-comb MIXSEL is a specialized SDL for which both the gain and the saturable absorber are integrated into the same semiconductor wafer. The semiconductor MIXSEL chip forms one cavity-end mirror, and the output coupler forms the other. In addition, two intracavity elements are used: first, an etalon to adjust the center wavelength, and second, a birefringent crystal for polarization splitting and dual-comb generation. The output coupler can be mounted on a piezo actuator to adjust the cavity length (and then the repetition rate). The dual-comb MIXSEL generates two collinear perpendicularly polarized (polarization indicated by circles and arrows) mode-locked pulse trains with a small difference in the pulse repetition frequency due to the difference in optical path length in the birefringent crystal. Thus, we obtain two collinear OFCs). A polarizing beam splitter (PBS), with an optical axis at an angle of $45^{\circ}$ to the polarization axes of the OFCs, combines both OFCs into the same polarization, which then optically interfere on a photodetector (PD). A microwave spectrum analyzer (MSA) directly measures the microwave frequency comb, which manifests as a comb structure $\left(\mathrm{comb}_{1}\right)$ between dc and the pulse repetition rates $f_{\text {rep }, 1}$ and $f_{\text {rep }, 2}$. The pink rectangle indicates the microwave comb. $\mathrm{dBc}$, decibels relative to the carrier. (B) The microwave frequency comb results from the optical interference on the PD between all the optical frequencies of the two collinear and combined OFCs with their offset in pulse repetition rate $\Delta f_{\text {rep }}$, which can be adjusted with the optical path length in the birefringent crystal. With this dual-comb principle, we obtain a direct link between the optical and the microwave frequencies. 
set (CEO) frequency (i.e., the frequency comb zero offset) for two separate OFCs. Therefore, four separate stabilization loops and two highly nonlinear processes for supercontinuum generation are required.

There is a concerted ongoing effort in the research community to find ways to simplify these systems (25-28). The detection of the CEO frequency with optical f-to-2f interferometry (2) requires a coherent octavespanning supercontinuum, which is very demanding on the laser performance and substantially reduces the power per mode. Unfortunately, most of the time the broad spectrum cannot be fully used for spectroscopy directly but needs to be filtered to avoid aliasing effects as described by the Nyquist- Shannon sampling condition (29). Therefore, very often it is sufficient to use a dual-comb source with a narrower optical spectrum centered at a wavelength which can be tailored to the region of interest (e.g., matching absorption lines of gases of interest).

We consider the dual-comb MIXSEL an ideal source for dual-comb spectroscopy. Generally, given an optical source at the required wavelength, key requirements for molecular spectroscopy include (i) as much power per line as possible, resulting in better signal-to-noise ratio and faster measurement time; (ii) sufficiently narrow line spacing to resolve the spectroscopic feature (e.g., the mode-locked repetition rate equals the optical line spacing); and (iii) sufficiently narrow optical linewidth, of the individual comb lines, to resolve the spectroscopic features. For example, the water linewidth near $960 \mathrm{~nm}$ is $\approx 10 \mathrm{GHz}$; obtaining five sampling points per line requires a comb spacing of 1 to $2 \mathrm{GHz}$. Typical comb linewidth should be less than 10 to $100 \mathrm{MHz}$. MIXSEL combs have been successfully demonstrated in the 1- to $100-\mathrm{GHz}$ regime (30), and in contrast to microcombs $(27,31)$ or quantum cascade laser combs $(32,33)$, the MIXSEL combs have shown even better performance in the 1- to $10-\mathrm{GHz}$ regime so far. For example, without any further active stabilization, a single 2-GHz MIXSEL comb has a comb line-spacing variation of only $\approx 2.5 \times 10^{-4}$ integrated over a measurement time of $10 \mathrm{~ms}(34)$, which is a longer measurement time than required for most dual-comb spectroscopy applications (typically $>1 \mathrm{~ms}$ to ideally $\approx 1 \mathrm{~ms}$ ). The simplicity of the dual-comb MIXSEL (Fig. 5.6A) allows for fundamental mode-locking in a simple straight linear cavity, and the operation wavelength can be adjusted 
with an intracavity etalon to a center wavelength of $968.3 \mathrm{~nm}\left(10,327 \mathrm{~cm}^{-1}\right)$ to match an absorption line of water vapor. The pulse repetition rate can be set by the laser-cavity length, which corresponds to about $9 \mathrm{~cm}$ in air for the 1.7-GHz comb spacing used in this work. For dual-comb mode-locked operation (13), we insert a birefringent crystal inside this linear cavity, which splits the cavity beam on the side toward the MIXSEL chip into two spatially separated and cross-polarized beams with slightly different optical-cavity round-trip path lengths, defining a small difference in the comb frequency spacing $\Delta f_{\text {rep }}$ of $4 \mathrm{MHz}$. This frequency difference can be adjusted by the thickness of the intracavity birefringent crystal (Fig. 5.6A). At the other side of the birefringent crystal, the two beams propagate collinearly but with perpendicular polarization, making them easy to separate, e.g., with a polarizing beam splitter (PBS). Therefore, both OFCs share the same optical components, pump laser, and electronics, leading to intrinsically strong mutual coherence.

When we first explored dual-comb modelocking (13), we encountered a very unexpected result. Cavity-length adjustments to stabilize one comb's spacing did not significantly affect the other comb's spacing. We were concerned that the incoherent pump laser might be responsible, which would have been a more serious problem for dual-comb spectroscopy. We therefore had to first resolve this mysterious behavior. We gained insight into the underlying physics by comparing semiconductor and Nd:YAG lasergain materials, leading to the conclusion that the time delay introduced by the fully saturated saturable absorber is responsible for decoupling the two combs in an uncorrelated but deterministic way that can be fully stabilized (35). We therefore could then proceed to application of dualcomb spectroscopy. Dual-comb spectroscopy of water vapor (Fig. 5.7) was first explored with the laser operating in free-running mode without any further active stabilization. A 40-m-long multipass cell was used to enhance the absorption, as the absorption cross section of water vapor at $968.3 \mathrm{~nm}$ is 4 orders of magnitude smaller than in the mid-IR. The two perpendicularly polarized collinear OFCs at the output of the dual-comb laser were combined into the same polarization with a PBS (Fig. 5.7A). One such combined dual-comb was directly detected with a photodetector (PD1) and a digitizer to serve as a reference (Fig. 5.7B, blue microwave 
A
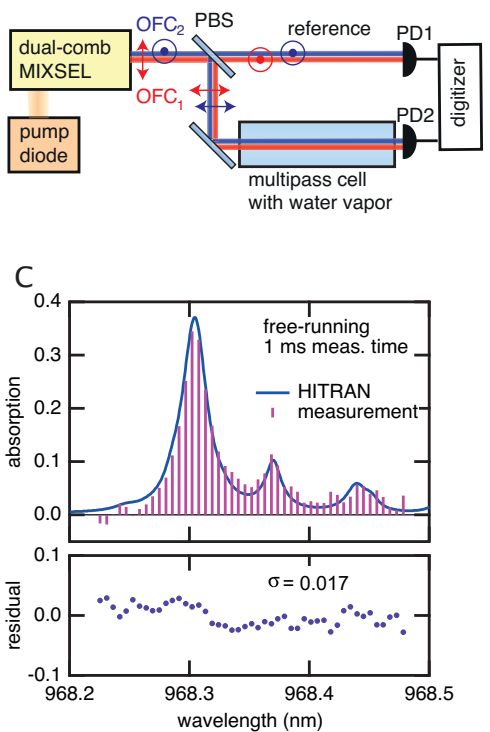

B
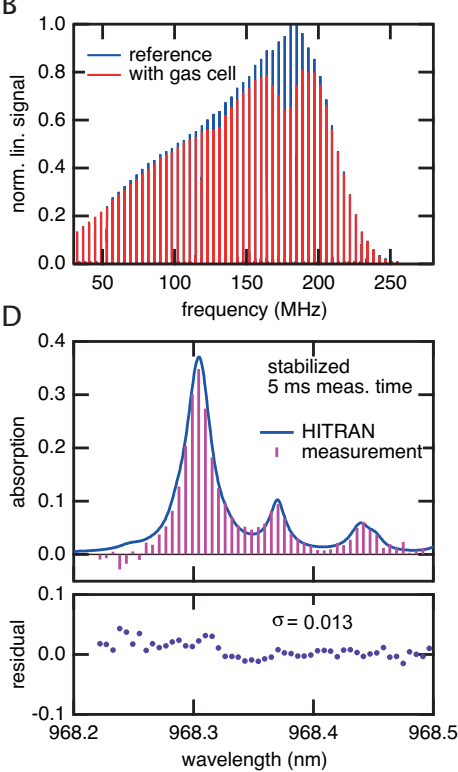

Figure 5.7: Dual-comb spectroscopy results. (A) Schematic of the experimental setup with two combined dual-comb OFCs. A reference microwave spectrum is measured with PD1, and the microwave spectrum after the multipass gas cell is simultaneously measured with PD2, making the setup insensitive to phase errors. (B) Microwave frequency combs for the reference measurement (blue) and the measurement with the gas cell (red), which is modulated because of the absorption of the water vapor. (C) Absorption measurement with the free-running system on water vapor and comparison with the HITRAN database, with the residual error shown below in purple. (D) Same measurements as in (C) with the stabilized microwave comb. 
spectrum). In analogy to Fourier spectrometers, the second frequency comb replaces the scanning mirror, which typically limits the scan rate to a few $\mathrm{Hz}$ in Michelson-type systems. With a frequency offset of $4 \mathrm{MHz}$ between the two combs, we have a minimum acquisition time of only $0.25 \mu$ s (Fig. 5.10) for the time-dependent interferograms detected by PD1. A simple Fourier transformation of the interferogram generates the spectrum shown in Fig. 5.7B. The other combined dual-comb after the PBS in Fig. 5.7A was sent through the air-filled multipass gas cell with a relative humidity of $60 \%$ at $36^{\circ} \mathrm{C}$ under ambient pressure and detected with PD2 (Fig. 5.7B, red microwave spectrum). The change introduced by the watervapor absorption was clearly visible; the absorption was calculated by taking the difference between the two microwave spectra and normalizing the difference with the reference comb (Fig. 5.7B, blue microwave spectrum) (Fig. 5.7C) (36). Here we directly use the absorption line of water to calibrate the absolute wavelength (36), which is a commonly used method for dual-comb spectroscopy. A comparison with the high-resolution transmission molecular absorption (HITRAN) database (37) simulation using the laboratory parameters shows excellent agreement even though no active stabilization of the dual-comb laser was used. The residual error, with a standard deviation of 0.017, is shown below these data (Fig. 5.7C). We could further improve the signal-to-noise ratio and prevent long-term drifts with a simple stabilization scheme. For example, a longer measurement duration of $5 \mathrm{~ms}$ with this active stabilization switched on shows even better agreement with the HITRAN data (Fig. 5.7D). For the stabilization scheme shown in Fig. 5.8A, both OFCs are superimposed in the same polarization on the photodetector (PD3) and analyzed with a microwave spectrum analyzer (MSA). The generated microwave frequency comb (Fig. 5.8B) results from the interference of the optical modes of the two beams, representing a direct link between the optical frequencies and the electronically accessible microwave regime (Fig. 5.6B). All lines of the microwave comb can be clearly resolved without any stabilization. With a measurement time of $10 \mathrm{~s}$, the comb lines fluctuate by only $\approx 200 \mathrm{kHz}$, shown here for a single comb line by holding the maximum of the trace for $10 \mathrm{~s}$ (blue line in Fig. 5.8C). This could be further improved with active stabilization. A single line (red line in Fig. 5.8B) of the comb was digi- 
tally filtered and locked to a stable electronic reference with an ultra-high frequency lock-in amplifier (UHFLI, Zurich Instruments). The error signal was corrected by current modulation to the multimode pump diode laser in the dual-comb MIXSEL. The stability of the comb line was substantially improved and the linewidth decreased to below $300 \mathrm{~Hz}$ (red line in Fig. $5.8 \mathrm{C}$ ). In addition to one comb line, $\Delta f_{\text {rep}}$, was stabilized by using a second input port of the UHFLI. The corresponding error signal was corrected by modulation of the cavity length by means of a piezo actuator- controlled output coupler.With both a stabilized comb line and a stabilized $\Delta f_{\text {rep }}$, all microwave comb lines were stabilized simultaneously, shown here for an arbitrarily chosen second comb line (green line in Fig. 5.8C) (36). Therefore, by applying only two feedback loops to signals that are measured directly with photodetectors without the need for any optical f-to-2f-interferometry (2), the full microwave frequency comb could be stabilized.

After characterizing the microwave frequency comb, we measured the absolute stability of the modes of the optical frequency combs. A single frequency laser was used to generate a beat signal with one of the modes of the two optical OFCs (Fig. 5.11). The strong beat frequency was measured at $100-\mathrm{kHz}$ resolution bandwidth (RBW) with the MSA (Fig. 5.8D). The small side peaks originate from the beat between the single-frequency laser and a mode of the other optical spectrum, because the suppression is limited by the suppression ratio of the PBS. We then measured the beat frequency during $10 \mathrm{~s}$ every $5 \mathrm{~ms}$ with a gate time duration of $1 \mathrm{~ms}$ with a frequency counter to characterize the fluctuations of the optical mode (Fig. 5.8D). The measurement confirmed that the short-term (few milliseconds) stability of the optical modes is sufficient even for the free-running system. The long-term (few seconds) stability of the optical modes drastically improved by turning on the stabilization of the microwave comb. The measurement was not limited by the stability of the single-frequency laser, which was characterized with a wavelength meter (gray line in Fig. 5.8D). For a measurement time of $1 \mathrm{~s}$, we could average more than $10^{6}$ interferograms, and the standard deviation of the beat frequency in the case of the stabilized microwave comb was below $800 \mathrm{kHz}(2.5 \mathrm{fm})$, which is more than 3 orders of magnitude smaller than the comb spacing of $1.76 \mathrm{GHz}$ 

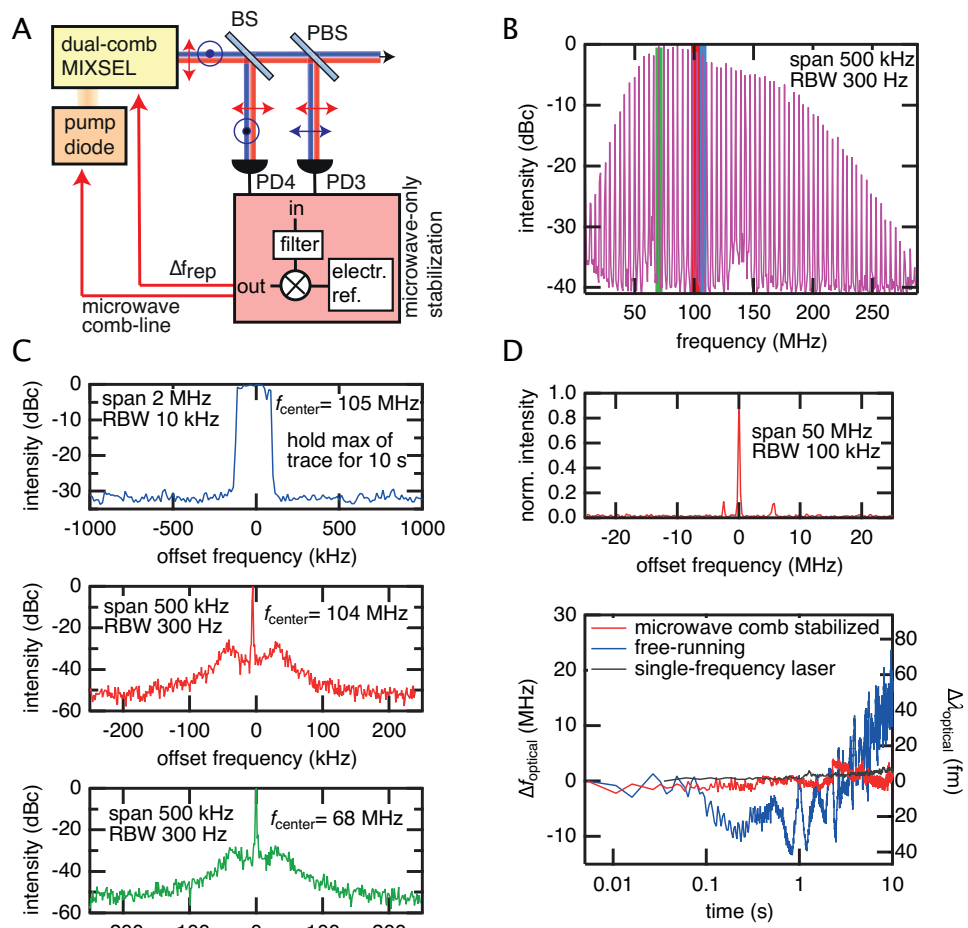

Figure 5.8: Stabilization of the microwave comb and optical mode stability. (A) Schematic of the stabilization setup. By using a beam splitter (BS), both OFCs (red and blue lines) are superimposed in cross-polarization on PD4 to prevent optical interference and to simply measure and stabilize the difference in pulse repetition

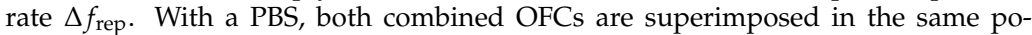
larization on PD3, where the microwave comb is measured and one of the comb lines is stabilized. Electr. ref., electronic reference. (B) Microwave spectrum of the free-running microwave comb1 (Fig. 5.6A) with a resolution bandwidth (RBW) of $10 \mathrm{kHz}$. (C) Magnification of a single microwave comb line. The blue line shows the fluctuations of a free-running comb line. The maximum of the trace is recorded for $10 \mathrm{~s}$ with a RBW of $10 \mathrm{kHz}$. The red line shows a stabilized comb line with a RBW of $300 \mathrm{~Hz}$. The green line demonstrates that all comb lines are simultaneously stabilized if the red line, and additionally $\Delta f_{\text {rep }}$, are stabilized (RBW $300 \mathrm{~Hz}$ ) (36). (D) Measurement of the absolute stability of the optical modes. The microwave spectrum shows the beat signal between the single-frequency laser and one optical comb line with a RBW of $100 \mathrm{kHz}$. The fluctuations of the beat frequency and the consequent fluctuations of the optical comb line are measured with a frequency counter over time in case of the free-running system (blue line) and with the microwave comb stabilization active (red line). The fluctuations of the single-frequency laser are measured with a wavelength meter (gray line). Stabilizing the microwave comb drastically improves the long-term stability of the optical comb lines. 
(5.5 pm). Because the line width of typical atmospheric absorption features is on the order of several gigahertz, an optical stability of $800 \mathrm{kHz}$ is more than sufficient for most applications (38), and no further additional stabilization of the optical spectrum is required.

The main trade-off with the free-running dual-comb MIXSEL approach compared to current systems is the reduced frequency comb bandwidth, requiring different lasers for applications with substantially different center wavelengths. However, semiconductor technology allows the OFC center wavelength to be tailored to the region of interest via band-gap engineering from the UV to the mid-IR and can be very cost effective due to its wafer scalability. We have observed a similar trend in biomedical imaging where the expensive, broadly tunable Ti:sapphire lasers are being replaced by much simpler optically pumped semiconductor disk lasers (SDLs) with a specific selection of operation wavelengths (39). In addition, we have shown that electrically pumped ultrafast SDLs are also possible with an average output power up to $50 \mathrm{~mW}$ to potentially further reduce complexity (40). We believe that the dual-comb MIXSEL approach has the potential to bring dual-comb spectroscopy from a laboratory environment to the field for a wide range of industrial applications.

Acknowledgements We thank M. Kroner and A. Imamoglu for lending us the single-frequency laser (Toptica DL pro); J. Deiglmayr and F. Merkt for the wavelength meter (High-Finesse WS-7); and G. Villares, M. Rösch, and J. Faist for the frequency counter (Agilent 53220A). The authors acknowledge the support of the technology and cleanroom facility at Frontiers in Research: Space and Time (FIRST) of ETH Zurich for advanced micro- and nanotechnology. This work was financed by the Swiss Confederation program Nano-Tera.ch, which was scientifically evaluated by the Swiss National Science Foundation (SNSF). S.M.L., B. W. Tilma, M. Mangold, C. A. Zaugg, A. Klenner, and U.K. are inventors on a patent application (WO 2016/049787 A1) held and submitted by ETH Zurich that covers dual-comb mode-locking. Data can be obtained by contacting U.K. at keller@phys.ethz.ch. 


\subsubsection{References and notes}

1. L.-S. Ma et al., Science 303, 1843-1845 (2004).

2. H. R. Telle et al., Appl. Phys. B 69, 327-332 (1999).

3. D. J. Jones et al., Science 288, 635-640 (2000).

4. A. Apolonski et al., Phys. Rev. Lett. 85, 740-743 (2000).

5. P. Del'Haye et al., Nature 450, 1214-1217 (2007).

6. J. Li, H. Lee, T. Chen, K. J. Vahala, Phys. Rev. Lett. 109, 233901 (2012).

7. D. Cotter, in Ultrafast Phenomena IV, D. H. Auston, K. B. Eisenthal, Eds. (Springer Series in Chemical Physics, Springer, 1984), vol. 38, pp. $78-80$.

8. M. J. W. Rodwell, D. M. Bloom, K. J. Weingarten, IEEE J. Quantum Electron. 25, 817-827 (1989).

9. A. Klenner, S. Schilt, T. Südmeyer, U. Keller, Opt. Express 22, 3100831019 (2014).

10. I. Hartl, H. A. McKay, R. Thapa, B. K. Thomas, J. Dong, M. E. Ferman, "GHz Yb-femtosecond-fiber laser frequency comb," paper presented at the Conference on Lasers and Electro-Optics (CLEO), CMN1, San Jose, CA, 2009.

11. A. Bartels, D. Heinecke, S. A. Diddams, Science 326, 681 (2009).

12. D. J. H. C. Maas et al., Appl. Phys. B 88, 493-497 (2007).

13. S. M. Link et al., Opt. Express 23, 5521-5531 (2015).

14. U. Keller, A. C. Tropper, Phys. Rep. 429, 67-120 (2006).

15. N. Schulz, J. M. Hopkins, M. Rattunde, D. Burns, J. Wagner, Laser Photonics Rev. 2, 160-181 (2008).

16. M. Guina, A. Härkönen, V.-M. Korpijärvi, T. Leinonen, S. Suomalainen, Adv. Opt. Technol. 2012, 265010 (2012).

17. B. W. Tilma et al., Light Sci. Appl. 4, e310 (2015).

18. A. Rahimi-Iman, J. Opt. 18, 093003 (2016). 
19. M. A. Gaafar et al., Adv. Opt. Photonics 8, 370-400 (2016).

20. S. Schiller, Opt. Lett. 27, 766-768 (2002).

21. I. Coddington, N. Newbury, W. Swann, Optica 3, 414-426 (2016).

22. A. Bartels et al., Rev. Sci. Instrum. 78, 035107 (2007).

23. I. Coddington, W. C. Swann, L. Nenadovic, N. R. Newbury, Nat. Photonics 3, 351-356 (2009).

24. K. O. Hill, Y. Fujii, D. C. Johnson, B. S. Kawasaki, Appl. Phys. Lett. 32, 647-649 (1978).

25. T. Ideguchi, A. Poisson, G. Guelachvili, N. Picqué, T. W. Hänsch, Nat. Commun. 5, 3375 (2014).

26. Y. Liu et al., Opt. Express 24, 21392-21398 (2016).

27. M.-G. Suh, Q.-F. Yang, K. Y. Yang, X. Yi, K. J. Vahala, Science 354, 600-603 (2016).

28. T. Yasui et al., Sci. Rep. 5, 10786 (2015).

29. C. E. Shannon, Proc. IEEE 86, 447-457 (1998) [reprinted from Proc. IRE 37, 10-21 (1949)].

30. M. Mangold et al., Opt. Express 22, 6099-6107 (2014).

31. T. J. Kippenberg, R. Holzwarth, S. A. Diddams, Science 332, 555-559 (2011).

32. G. Villares, A. Hugi, S. Blaser, J. Faist, Nat. Commun. 5, 5192 (2014).

33. G. Villares et al., Appl. Phys. Lett. 107, 251104 (2015).

34. M. Mangold et al., IEEE Photonics J. 6, 1-9 (2014).

35. S. M. Link, A. Klenner, U. Keller, Opt. Express 24, 1889-1902 (2016).

36. Materials and methods are available as supplementary materials.

37. L. S. Rothman et al., J. Quant. Spectrosc. Radiat. Transf. 130, 4-50 (2013).

38. P. J. Schroeder et al., Proc. Combust. Inst. 36, 4565-4573 (2017).

39. G. Vicidomini et al., Nat. Methods 8, 571-573 (2011). 
40. C. A. Zaugg et al., Appl. Phys. Lett. 104, 121115 (2014).

41. D. Waldburger et al., Optica 3, 844-852 (2016).

\subsubsection{Supplementary materials}

\section{Experimental setup}

The dual-comb MIXSEL benefits from the simple straight linear cavity. The linear cavity is defined by the MIXSEL chip and an output coupler (radius of curvature $100 \mathrm{~mm}$, transmission $0.5 \%$ ) as the two end mirrors (Fig. 5.6A). It is straightforward with this cavity design to apply the concept of polarization duplexing by inserting a 2-mm thick, wedged birefringent $\mathrm{CaCO} 3$ crystal into the cavity to split the one cavity beam into two cross-polarized and spatially separated beams (13). Both cavity spots on the MIXSEL chip are pumped under an angle of $45^{\circ}$ with a commercial multimode pump diode at $808 \mathrm{~nm}$, which is split with a 50:50 beam splitter (BS). Both optical spectra are tuned with an intracavity etalon to a center wavelength of $968.3 \mathrm{~nm}$ to match an absorption line of water vapor. The pulse duration is $17.6 \mathrm{ps}$ and $18.6 \mathrm{ps}$ and the average output power is $60 \mathrm{~mW}$ and $78 \mathrm{~mW}$ for the $\mathrm{p}$ - and s-polarized beam, respectively (Fig. 5.9). The temperature of the MIXSEL chip is kept constant with a Peltier element at $14{ }^{\circ} \mathrm{C}$.

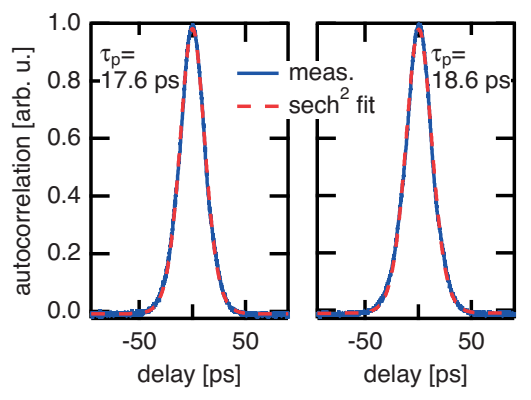

Figure 5.9: Second-harmonic autocorrelation of the two cross-polarized laser beams. 


\section{Absorption calculation}

The time-dependent interferograms (Fig. 5.10) of the reference arm and the multi-pass cell arm are recorded simultaneously with two photodetectors (PD1 and PD2) with a 5-GHz bandwidth and a high-speed oscilloscope with a 1-GHz bandwidth (Fig. 5.7A). The Fourier transformations of the two time-dependent interferograms result in the two microwave combs (Fig. 5.7B) from which the area below each peak is calculated with numeric integration. The absorption at each peak position $A_{\mathrm{i}}$ is then calculated by subtracting the area of the $\mathrm{i}^{\text {th }}$ peak of the reference spectrum $a_{\mathrm{i}, \text { ref }}$ from the area of the corresponding peak in the spectrum measured with the multipass gas cell $a_{\mathrm{i}, \mathrm{MPC}}$. Dividing this value by $a_{\mathrm{i}, \text { ref }}$ gives the absorption:

$$
a_{\mathrm{i}}=\frac{a_{\mathrm{i}, \mathrm{ref}}-a_{\mathrm{i}, \mathrm{MPC}}}{a_{\mathrm{i}, \mathrm{ref}}}
$$

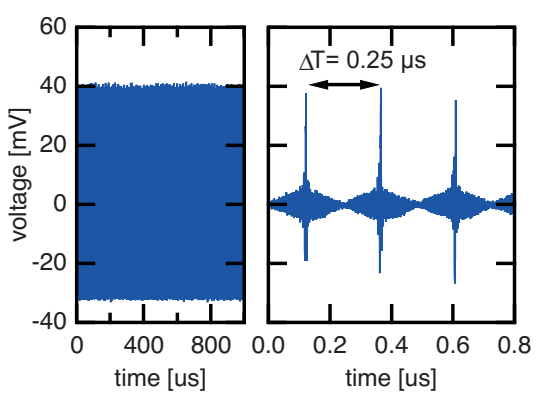

Figure 5.10: Signal from optical interference of the two combined OFCs recorded with the PD in the time domain showing time-dependent interferograms over a time span of $1 \mathrm{~ms}$ and $0.8 \mu \mathrm{s}$. Whenever one pulse of each OFC hits the detector at the same time a strong burst peak is recorded. The bursts are repeated every $0.25 \mu \mathrm{s}$, corresponding to the inverse of the difference in pulse repetition rate $\Delta f_{\text {rep }}$ of $4 \mathrm{MHz}$.

The wavelength axis can be calibrated using the optical mode spacing and $\Delta f_{\text {rep. }}$. For the absolute wavelength position one could take a single frequency laser with known frequency or an atomic transition. Here we 
directly use the absorption line of water to calibrate the absolute wavelength, which is a commonly used method for dual-comb spectroscopy. The microwave axis (Fig. 5.7B) has to be inverted compared to the wavelength axis (Fig. 5.7C and D) in this case. This depends on which of the two combs between DC and the pulse repetition rates (comb or $^{\circ} \mathrm{comb}_{2}$ in Fig. 5.6A) have been used for the measurement because they mirror each other. Measuring the comb at lower microwave frequencies is preferred, since less bandwidth is required for the PDs and the oscilloscope. The HITRAN simulation is calculate with the HITRAN database taking the temperature, pressure, relative humidity and path length as input parameters (Fig. 5.7C and D).

\section{Full stabilization of the microwave comb}

Stabilizing only a single line of the microwave comb is not sufficient to stabilize the full comb since it depends on two parameters: the difference in repetition rate $\Delta f_{\text {rep }}$ and the difference in carrier envelope offset (CEO) frequency $\Delta f_{\mathrm{CEO}}$. Therefore two feedback loops are required. The comb lines are a result of the beating of the optical modes of the two combined collinear OFCs. The red line (Fig. 5.8B) $f_{\text {red }}$ is given by

$$
f_{\text {red }}=\left(N_{2} \cdot f_{\text {rep }, 2}+f_{\mathrm{CEO}, 2}\right)-\left(N_{1} \cdot f_{\text {rep }, 1}+f_{\mathrm{CEO}, 1}\right) \approx \text { const },
$$

where $N_{1}$ and $N_{2}$ are integers. This beat frequency is kept constant within the limits of the feedback loop. The expression can be re-written as

$$
f_{\text {red }}=\Delta f_{\text {CEO }}+\left(N_{2}-N_{1}\right) \cdot f_{\text {rep }, 1}+N_{2} \cdot \Delta f_{\text {rep }} \approx \text { const. }
$$

with $\Delta f_{\text {rep }}$ given by

$$
\Delta f_{\text {rep }}=f_{\text {rep }, 2}-f_{\text {rep }, 1}
$$

and $\Delta f_{\mathrm{CEO}}$ described by

$$
\Delta f_{\mathrm{CEO}}=f_{\mathrm{CEO}, 2}-f_{\mathrm{CEO}, 1}
$$

The green line (Fig. 5.8B) $f_{\text {green }}$ of the comb is given by

$f_{\text {green }}=\left(\left(N_{2}+m\right) \cdot f_{\text {rep }, 2}+f_{\mathrm{CEO}, 2}\right)-\left(\left(N_{1}+m\right) \cdot f_{\text {rep }, 1}+f_{\mathrm{CEO}, 1}\right) \approx$ const, 
where $m$ is an integer counting the number of lines between the red and the green line. We can re-write $f_{\text {green }}$ and express it as a function of $f_{\text {red, }}$

$$
\begin{aligned}
f_{\text {green }} & =\Delta f_{\mathrm{CEO}}+\left(N_{2}-N_{1}\right) \cdot f_{\text {rep }, 1}+N_{2} \cdot \Delta f_{\text {rep }}+m \cdot \Delta f_{\text {rep }} \\
& \approx \text { const }+m \cdot \Delta f_{\text {rep }}
\end{aligned}
$$

from which we can conclude that in order to stabilize the green line, we need to stabilize both $\Delta f_{\text {rep }}$ and one comb line, which are directly detected with PD3 and PD4 (Fig. 5.8A). We use PD3 and PD4 to obtain a better signal-to-noise ratio, but in principle it would also be sufficient to take the signal from PD1 for the stabilization and therefore only use two detectors in total. Since Eq. 5.12 is true for any integer $m$, it is true for all microwave comb lines and therefore the full comb is stabilized simultaneously when both feedback loops are applied. Even though the microwave comb is fully stabilized, both optical spectra could fluctuate the same amount in the same direction, e.g. if the two CEO frequencies are changing by the exact same amount, which would not be visible in the microwave spectrum and accordingly could not be prevented by the two feedback loops. Therefore, we characterize the absolute stability of the modes of the optical frequency combs as well (Fig. 5.11) (Fig 5.8D).

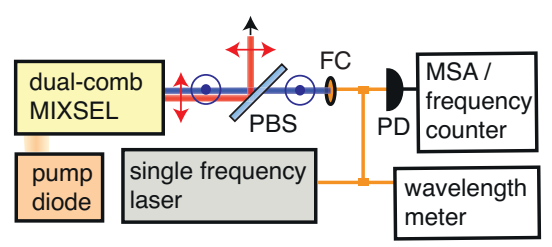

Figure 5.11: Measurement setup for the absolute stability of the optical modes of one of the two optical frequency combs (OFCs). A commercial highly stable single frequency laser (Toptica DL pro) is used to generate a beat frequency with one of the modes of one OFC on the photo detector (PD). This beat frequency is measured with a microwave spectrum analyzer (MSA) and a frequency counter. To make sure that the measurement is not limited by the fluctuations of the single frequency laser, its frequency is characterized with a wavelength meter. PBS polarizing beam splitter (polarizer axis turned such that the OFCs are spatially separated), FC fiber coupling. 



\section{Pulse shortening strategies for semiconductor disk lasers}

The last chapter shows, that the dual-comb MIXSEL performs very well for gas sensing using the dual-comb spectroscopy approach. The main limitations for this first proof-of-concept demonstration are the center wavelength and the optical bandwidth. The center wavelength is with $968 \mathrm{~nm}$ in a regime where very little absorption features of molecules are situated. An outlook on potential strategies to shift the center wavelength to more exciting spectroscopic regions will be given in chapter 8 .

The optical bandwidth scales with the the pulse duration - the shorter the pulses, the larger the optical bandwidth. Therefore, it is a strong ongoing effort to reduce the pulse durations of our SDLs. Previous simulations [50] have shown that the following parameters are crucial to obtain short pulses from SDLs:

- Low, spectrally flat and broad field intensity enhancement factor to support a large gain bandwidth and a high saturation fluence for the gain

- Broadband and flat GDD close to zero but positive

- Fast recovery time for the saturable absorber

- An increased field intensity enhancement for the absorber compared to the gain to reduce the saturation fluence of the absorber 
Following these guidelines enabled sub-200 fs from a MIXSEL [27] and sub-100 fs for a SESAM-modelocked VECSEL. This was the first time that sub-100 fs were reached with a fundamentally modelocked SDL. The broad optical spectrum of $17 \mathrm{~nm}$ FWHM corresponds to a usable $-30 \mathrm{~dB}$ bandwidth of more than $50 \mathrm{~nm}$ (Fig. 6.4), which is already a very promising bandwidth for dual-comb spectroscopy. The details of these recordshort pulses from a SDL are presented in the following Optica publication [21]. (@ 2016 Optical Society of America. One print or electronic copy may be made for personal use only. Systematic reproduction and distribution, duplication of any material in this paper for a fee or for commercial purposes, or modifications of the content of this paper are prohibited.)

\subsection{High-power $100 \mathrm{fs}$ semiconductor disk lasers}

Dominik Waldburger, ${ }^{1 *}$ Sandro M. Link, ${ }^{1}$ Mario Mangold, ${ }^{1}$ Cesare G. E. Alfieri, ${ }^{1}$ Emilio Gini, ${ }^{2}$ Matthias Golling, ${ }^{1}$ Bauke W. Tilma, ${ }^{1}$ and Ursula Keller ${ }^{1}$

${ }^{1}$ Department of Physics, Institute for Quantum Electronics, ETH Zürich, 8093 Zürich, Switzerland.

${ }^{2}$ FIRST Center for Micro- and Nanoscience, ETH Zürich, 8093 Zürich, Switzerland.

Optically pumped passively modelocked semiconductor disk lasers (SDLs) provide superior performance in average output power, a broad range of operation wavelengths, and reduced complexity. Here, we present record performance with high average power and pulse durations as short as $\mathbf{1 0 0} \mathrm{fs}$ with a semiconductor saturable absorber mirror (SESAM) modelocked vertical external-cavity surface-emitting laser (VECSEL) at a center wavelength of $1034 \mathrm{~nm}$. A comprehensive pulse characterization confirms fundamental modelocking with a close to transform-limited output pulse of 128 fs and with negatively chirped output pulses as short as $107 \mathrm{fs}$, which are externally compressed to 96 fs with a single path through a 2-mm-thick ZnSe plate. For the "96 fs result" the pulse repetition rate is $1.6 \mathrm{GHz}$, the average output power is $100 \mathrm{~mW}$, and the pulse peak power is $560 \mathrm{~W}$. The transform-limited 
optical spectrum could in principle support pulses as short as $65 \mathrm{fs}$ with higher order dispersion compensation. We measured the most relevant spectral and nonlinear VECSEL and SESAM parameters and used them as input parameters for our pulse formation simulations. These simulations agree well with our experimental results and provide an outlook for further performance scaling of ultrafast SDL technology.

\subsubsection{Introduction}

Passive modelocking of optically pumped semiconductor disk lasers (SDLs) [1] using semiconductor saturable absorber mirrors (SESAMs) [2] has de-monstrated impressive progress during the past 10 years, as reviewed recently [3]. The optically pumped vertical external-cavity surface-emitting laser (VECSEL) [4] was the first laser in the family of semiconductor disk lasers to demonstrate significant power scaling by superior heat removal in a thin disk geometry. The optically pumped VECSEL is an efficient mode converter that uses low-coherent high-power diode pump arrays and benefits from semiconductor bandgap engineering to support emission wavelengths ranging from UV to mid-IR (i.e., $391 \mathrm{~nm}$ up to $5.3 \mu \mathrm{m}$ ) [5,6]. The highest continuous wave (cw) output power is $\approx 20 \mathrm{~W}$ in a Gaussian mode profile [7-9] and $106 \mathrm{~W}$ in multimode operation [10] at an emission wavelength around $\approx 1 \mu \mathrm{m}$ using InGaAs quantum well gain on GaAs/AlGaAs Bragg reflectors. Here, we present record power performance with SESAM-modelocked VECSELs generating pulse durations in the $100 \mathrm{fs}$ regime (Fig. 6.1).

We have observed a strong trade-off between short pulse durations and average power or pulse peak power (Fig. 6.1). Fundamental physical processes, such as non-equilibrium kinetic hole burning [12], can partially explain this, but the full complex interplay between the gain and absorber dynamics in these high-Q SDLs are not yet fully understood. To date, the shortest pulses have been $107 \mathrm{fs}$, but with only $3 \mathrm{~mW}$ of average output power [13] and $60 \mathrm{fs}$ in a pulse train within a picosecond pulse envelope [14]. The highest pulse peak power of up to $4.35 \mathrm{~kW}$ was obtained with significantly longer pulses of $400 \mathrm{fs}$ duration [15].

Peak power scaling by reducing the pulse repetition rate has been 


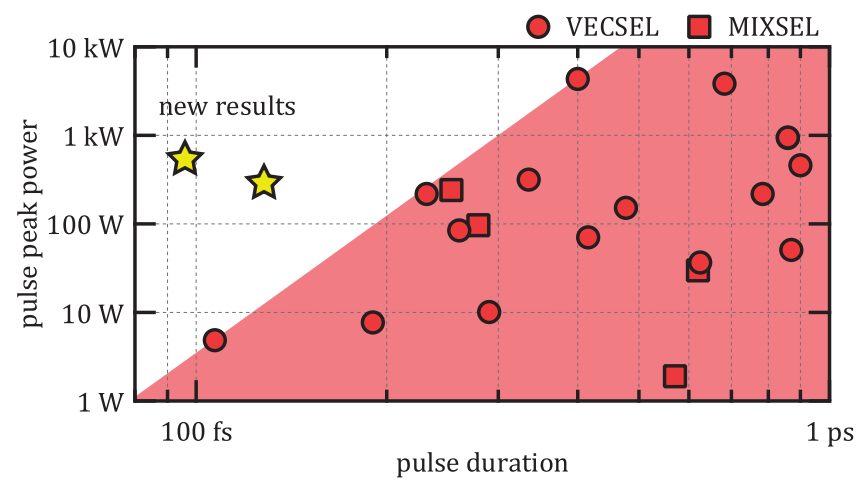

Figure 6.1: Overview of the pulse peak power versus pulse duration of the stateof-the-art femtosecond SDLs operating at a center wavelength around $1 \mu \mathrm{m}$ using InGaAs gain layers $[3,11]$. The yellow stars indicate the two new milestone results presented in this paper.

successfully demonstrated with diode-pumped solid-state lasers [16]. This approach is less attractive for diode-pumped semiconductor lasers because the highly inverted quantum well gain has a lifetime of only a few hundred picoseconds, which currently limits the pulse repetition rate of quantum well VECSELs to above $100 \mathrm{MHz}$ without the use of a more elaborated multi-gain-pass cavity [17,18]. Quantum dot gain SDLs [19-21] have a longer upper-state lifetime and may offer better performance in the future.

Many applications greatly benefit from a combination of femtosecond pulses with high pulse peak power to drive nonlinear optical processes, such as two-photon absorption for multiphoton microscopy [22] or supercontinuum generation for frequency comb applications [23-25]. The standard method to detect and stabilize the carrier-envelope offset (CEO) frequency in a self-referencing scheme is based on f-to-2f interferometry [26], for which a coherent octave-spanning spectrum is required. Typically the combination of sub-100-fs pulses with kilowatt pulse peak power was required for coherent supercontinuum generation in photonic crystal fibers [27], but supercontinuum generation in silicon nitride waveguides strongly reduces this requirement [28].

The limited upper-state lifetime of semiconductor lasers makes them 
ideally suited for gigahertz pulse repetition rates and the high gain crosssection eliminates Q-switching instabilities, typically a challenge with SESAM-modelocked diode-pumped ion-doped solid-state lasers [29,30]. The gigahertz repetition rate can be beneficial for frequency comb applications, since the comb lines have a larger frequency spacing with more power per mode and therefore provide a better signal-to-noise ratio (SNR). Additional benefits are a faster data acquisition or a faster data transmission in telecommunication applications [31]. To date, ultrafast SDLs have been demonstrated up to $100 \mathrm{GHz}$ [32].

Here, we present two new milestone results for femtosecond ultrafast SDLs (shown in yellow in Fig. 6.1). First, our "128 fs result" with a pulse duration of $128 \mathrm{fs}$, a pulse repetition rate of $1.8 \mathrm{GHz}$, an average power of $80 \mathrm{~mW}$, and, therefore, a pulse peak power of $303 \mathrm{~W}$, slightly improved compared to [33]. Second, we present our "96 fs result" with a pulse duration of $96 \mathrm{fs}$, a pulse repetition rate of $1.6 \mathrm{GHz}$, an average power of $100 \mathrm{~mW}$, and, therefore, a pulse peak power of $560 \mathrm{~W}$. These are, to the best of our knowledge, the shortest pulses of a fundamental modelocked SDL. To achieve pulse duration of $96 \mathrm{fs}$, the slightly negatively chirped $107 \mathrm{fs}$ output pulses have been compressed with a single path through a 2-mm-thick zinc selenide plate.

We used a newly optimized VECSEL structure following the guidelines based on numerical pulse formation simulations [34]. This VECSEL structure has a larger gain saturation fluence and a slightly positive but flat group delay dispersion (GDD) over a larger gain bandwidth. In addition, a fast SESAM with a low saturation fluence was required. In a similar approach Head et al. [35] presented preliminary results of a high-power sub-200-fs VECSEL. We did not include this result in our overview graph because the VECSEL chip got damaged before fundamental modelocking was confirmed. Higher average power can easily be obtained with multiple pulses per cavity round-trip.

We measured most laser and absorber parameters (Table 6.1) [36,37], which were used as input parameters for our numerical pulse formation simulation and obtained a good agreement. This allows us to give an outlook for further performance scaling at the end of this paper. 


\subsubsection{Laser design}

The laser cavity is arranged in a simple V-shaped cavity where the output coupler and the SESAM form the end mirrors, and the VECSEL chip is used as a folding mirror, as shown in Fig. 6.2. For the "96 fs result" [Fig. 6.2(a)], we changed the V-shaped cavity such that we more strongly saturate the absorber (i.e., beam radius in the gain estimated from ABCDmatrix calculations is increased from 186 to $192 \mu \mathrm{m}$, and in the absorber the beam radius is decreased from 100 to $83 \mu \mathrm{m}$; Table 6.1) and obtained more balanced cavity legs $\mathrm{L}_{1}$ and $\mathrm{L}_{2}$ (Fig. 6.2), which is clearly preferred for better gain recovery. In addition, for the "96 fs result", we inserted a 1mm-thick fused silica Brewster plate (Infrasil 302, 300 wedged; wzw-optic AG) between the VECSEL and the SESAM for more stable operation in linear polarization and shorter pulses. The wedged plate strongly reduces etalon effects and introduces additional positive dispersion inside the cavity. The VECSEL is pumped under an angle of $45^{\circ}$ with a $35 \mathrm{~W}, 808 \mathrm{~nm}$ spatial multimode diode laser (LIMO; Lissotschenko Mikrooptik GmbH), with an elliptical shaped beam such that on the VECSEL chip surface the pump beam is circular with a radius of $177 \mu \mathrm{m}$. For the "96 fs result"we used a pump power of $21 \mathrm{~W}$ and for the " 128 fs result" we used $18.1 \mathrm{~W}$. The VECSEL and the SESAM are temperature stabilized with water-cooled Peltier elements to $1^{\circ} \mathrm{C}\left(-10^{\circ} \mathrm{C}\right)$ and $37^{\circ} \mathrm{C}\left(24^{\circ} \mathrm{C}\right)$, respectively for the "96 fs result" ("128 fs result").

\section{VECSEL}

The VECSEL structure was grown on a metalorganic vapor phase epitaxy (MOVPE) machine. For flip-chip bonding, the structure was grown in reversed order on a 600- $\mu$ m-thick, (100)-oriented GaAs substrate. The structure is divided into a distributed Bragg reflector (DBR), an active region, and a multipurpose antireflection (AR) top coating, as shown in Fig. 6.2(c). The active region is grown at a lower substrate temperature of $660^{\circ} \mathrm{C}$, while the DBR and the AR top coating are grown at $750^{\circ} \mathrm{C}$.

The DBR consists of 23.5 pairs of GaAs/AlAs quarter-wave layers, which balances the trade-off between sufficient DBR reflectivity and high thermal conductivity to the underlying heat spreader. The center wavelength is designed to be at $1030 \mathrm{~nm}$. 

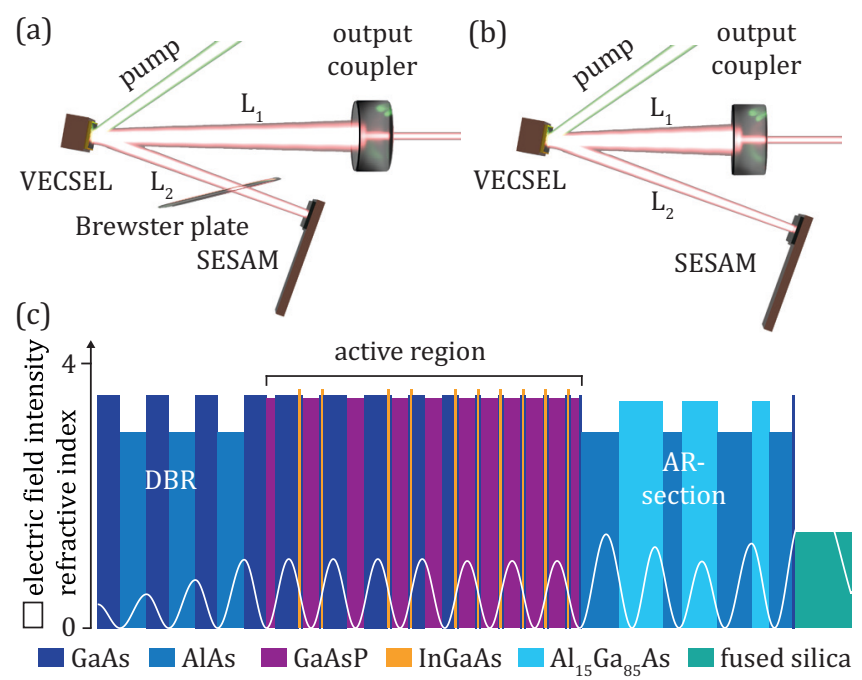

Figure 6.2: V-shaped cavity design with SESAM and output coupler as end mirrors and the VECSEL gain chip as a folding mirror. The output coupler has a $0.9 \%$ transmission and a radius of curvature of $100 \mathrm{~mm}$. The folding angle is $20^{\circ}$. The 1mm-thick fused silica Brewster plate was only used for the "96 fs result." (a) Cavity for the "96 fs result": $L_{1}=4.8 \mathrm{~cm}$ and $L_{2}=4.2 \mathrm{~cm}$. (b) Cavity for the "128 fs result": $L_{1}=3.4 \mathrm{~cm}$ and $L_{2}=4.8 \mathrm{~cm}$. (c) VECSEL layer stack, with a DBR, a straincompensated active region with $10 \mathrm{InGaAs}$ quantum wells, and a multi-purpose AR section. The center laser wavelength is designed to be $1030 \mathrm{~nm}$, and the white curve indicates the corresponding standing electric field intensity (normalized to 4 outside the structure).

The active region contains five pairs of strain-compensated InGaAs quantum wells. The compressively strained, 8.8-nm-thick $\operatorname{In}_{0.193} \mathrm{Ga}_{0.807} \mathrm{As}$ quantum wells are embedded in GaAs and compensated by $54 \mathrm{~nm}$ tensile strained $\mathrm{GaAs}_{0.933} \mathrm{P}_{0.067}$ layers. The quantum well pairs are separated by $64 \mathrm{~nm}$ and are placed symmetrically around the node or the anti-node of the standing electric field intensity. The spacing is optimized to obtain a low and spectrally flat field intensity enhancement factor of 0.52 [Fig. 6.3(a)]. The field intensity enhancement factor is defined as the field intensity inside the quantum wells averaged over all quantum wells and is normalized to the incoming field intensity (i.e., a 100\% reflector results in a field intensity of 4) [38]. The spectrally flat field intensity enhance- 
ment avoids structural gain bandwidth restrictions, and the lower field intensity enhancement is expected to increase the gain saturation fluence at the expense of available gain. The pump absorbing GaAs layers become thicker with increasing penetration of the pump light [Fig. 6.2(c)], and the AR coating is designed for no absorption. The AR section consists of 3.5 pairs of $\mathrm{Al}_{0.15} \mathrm{Ga}_{0.85} \mathrm{As} / \mathrm{AlAs}$ followed by a fused $\mathrm{SiO}_{\mathrm{x}} \mathrm{N}_{\mathrm{y}}$ dielectric layer and is optimized for a reduced pump reflectivity, a broadband flat GDD, and a close to zero but positive GDD.

The semiconductor structure is cleaved in $4.5 \mathrm{~mm} \times 4.5 \mathrm{~mm}$ pieces and is then flip-chip bonded onto a 1-mm-thick chemical vapor deposited diamond heat spreader with a high thermal conductivity exceeding $2000 \mathrm{~W} \mathrm{~m}^{-1} \mathrm{~K}^{-1}$ [39]. After soldering and wet etching, the final $\mathrm{SiO}_{\mathrm{x}} \mathrm{N}_{\mathrm{y}}$ layer is deposited using plasma-enhanced chemical vapor deposition (PECVD; Oxford Instruments).

The two modelocking results have been achieved with chips from different processing runs resulting in different $\mathrm{SiO}_{\mathrm{x}} \mathrm{N}_{\mathrm{y}}$ layer thicknesses and so different GDD. The GDD of the VECSELs is measured at $20^{\circ} \mathrm{C}$ without optical pumping [40]. The measured GDD curves [Fig. 6.3(b)] are not flat but have in comparison to prior results [23] a much smaller deviation of $\pm 100 \mathrm{fs}^{2}$. The drop in GDD at $1015 \mathrm{~nm}$ is due to the absorption of the quantum wells. The nonuniform PECVD deposition allows us to further optimize the GDD by scanning the position on the processed chip.

\section{SESAM}

The SESAM was grown on a molecular beam epitaxy (MBE) machine. A single InGaAs quantum well absorber is grown on top of a 30 pair GaAs/AlAs DBR. To achieve a fast recovery time, the absorber is grown at a low temperature of $260^{\circ} \mathrm{C}$ and is embedded in AlAs barriers. The field intensity enhancement factor of the antiresonant structure can be engineered by PECVD deposition of a $\mathrm{SiN}_{\mathrm{x}}$ top coating. An increased field intensity enhancement gives rise to a lower saturation fluence and an increased modulation depth [38]. For the "96 fs result", we reduced the mode size for a stronger saturation and increased the modulation depth with a higher temperature (i.e., moving from a heat-sink temperature of $24^{\circ} \mathrm{C}$ to $37^{\circ} \mathrm{C}$ ). All SESAM parameters are summarized in Table 6.1. 

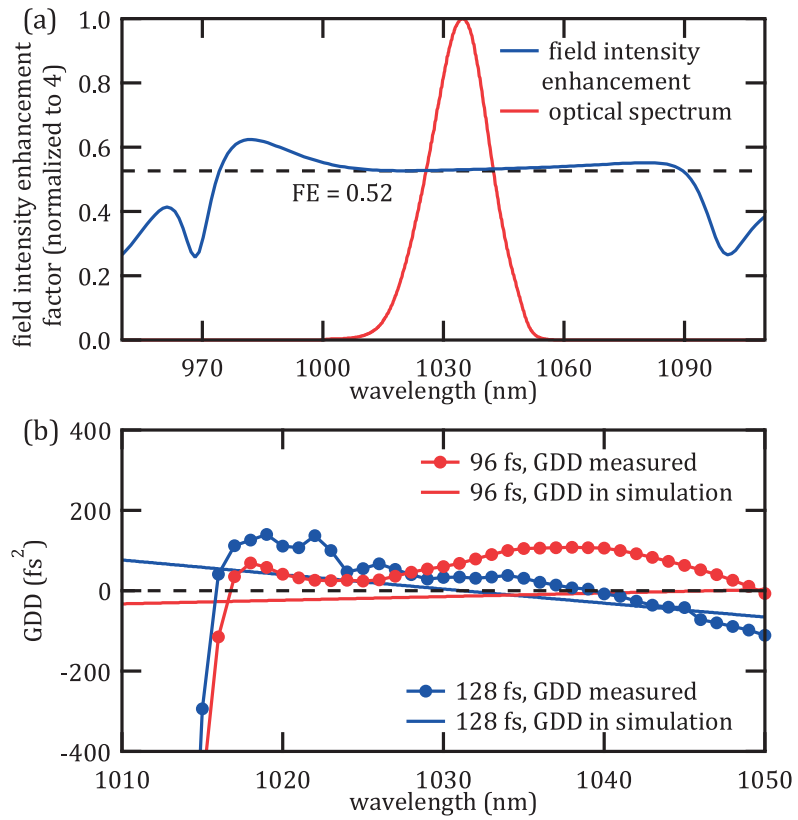

Figure 6.3: (a) Average field intensity in the gain quantum wells of the VECSEL (blue) normalized to the incoming field intensity (i.e., a 100\% reflector results in a field intensity of 4) and the measured optical spectrum (red). (b) Measured GDD of the "unpumped" VECSEL structure at normal incidence and at room temperature (circles). For simulation of the modelocking results, we used the dispersion (both second and third order) as an adjustable parameter (Table 6.1). The dispersion explain-ing our pulse durations is shown for the "128 fs result" (blue line) and the "96 fs result" (red line).

\subsubsection{Modelocking result}

A careful and extended pulse characterization has to be performed to prove clean and stable fundamental modelocking. An incomplete or limited characterization may incorrectly suggest fundamental modelocking and may result in much higher average output powers [11]. In the following, the two modelocking results are presented. For the "96 fs result", the initially negatively chirped $107 \mathrm{fs}$ output pulses are externally compressed by a single path through a 2-mm-thick zinc selenide plate (Eksma Optics) introducing a positive GDD of $\approx 1350 \mathrm{fs}^{2}$. 
Table 6.1: Complete list of input parameters used for the pulse formation simulation. (All non-italic parameters have been directly measured. If different, we show the parameters for the two modelocking results as follows: $96 \mathrm{fs}$ input parameter/128 fs input parameter.)

\begin{tabular}{|c|c|c|c|c|c|}
\hline Cavity & & VECSEL & & SESAM & \\
\hline Output coupler & $0.9 \%$ & $\begin{array}{l}\text { Saturation } \\
\text { fluence }\end{array}$ & $40 \mu \mathrm{J} / \mathrm{cm}^{2}$ & $\begin{array}{l}\text { Saturation } \\
\text { fluence }\end{array}$ & $\begin{array}{c}4.3 / \\
5.4 \mu \mathrm{J} / \mathrm{cm}^{2}\end{array}$ \\
\hline Other losses & $0.8 \%$ & $\begin{array}{l}\text { Recovery } \\
\text { time }\end{array}$ & $3 \mathrm{~ns}$ & $\begin{array}{l}\text { Fast recovery } \\
\text { time }\end{array}$ & $560 \mathrm{fs}$ \\
\hline Repetition rate & $\begin{array}{c}1.63 / \\
1.81 \mathrm{GHz}\end{array}$ & $\begin{array}{l}\text { Linewidth } \\
\text { enhancement } \\
\text { factor }\end{array}$ & 3 & $\begin{array}{l}\text { Slow recovery } \\
\text { time }\end{array}$ & $5.5 \mathrm{ps}$ \\
\hline $\begin{array}{l}\text { Center } \\
\text { wavelength }\end{array}$ & $\begin{array}{c}1034 / \\
1033 \mathrm{~nm}\end{array}$ & $\begin{array}{l}\text { Small signal } \\
\text { gain }\end{array}$ & $2.9 \%$ & $\begin{array}{l}\text { Aplitude of } \\
\text { slow } \\
\text { component }\end{array}$ & 0.25 \\
\hline $\begin{array}{l}\text { Round-trip } \\
\text { GDD }\end{array}$ & $-12 / 12 \mathrm{fs}^{2}$ & $\begin{array}{l}\text { Gain } \\
\text { FWHM }\end{array}$ & $50 \mathrm{~nm}$ & $\begin{array}{l}\text { Linewidth } \\
\text { enhancement } \\
\text { factor }\end{array}$ & 2 \\
\hline \multirow[t]{2}{*}{$\begin{array}{l}\text { Round-trip } \\
\text { TOD }\end{array}$} & $\begin{array}{l}-500 / \\
620 \mathrm{fs}^{3}\end{array}$ & $\begin{array}{l}\text { Beam } \\
\text { radius }\end{array}$ & $\begin{array}{c}192 / \\
185 \mu \mathrm{m}\end{array}$ & $\begin{array}{l}\text { Modulation } \\
\text { depth }\end{array}$ & $2.03 / 1.37 \%$ \\
\hline & & & & Beam radius & $83 / 100 \mu \mathrm{m}$ \\
\hline
\end{tabular}

The detailed pulse characterization is summarized in Figs. 6.4 and 6.5. The pulse duration is measured with a home-built second harmonic generation (SHG) frequency-resolved optical gating (FROG) apparatus. The measured SHG-FROG spectrogram is reconstructed with a grid size of 512 by 512 points [Fig. 6.4(a)] and 1024 by 1024 points [Fig. 6.5(a)]. The retrieved pulse durations agree well with a more standard SHG-based autocorrelator (FR-103MN; Femtochrome Research, Inc.). The intensity auto-correlations are in excellent agreement with a $\operatorname{sech}^{2}$ pulse shape [Figs. 6.4(d) and 6.5(d)], and long-span autocorrelation scans show no additional side pulses or pedestals [inset in Figs. 6.4(d) and 6.5(d)]. Figure 6.5(d) shows the autocorrelation of both the $107 \mathrm{fs}$ output pulses and the compressed 96 fs pulses. The following pulse characterizations are identical for the $107 \mathrm{fs}$ and the $96 \mathrm{fs}$ pulses. The smooth modelocked spectrum overlaps with the retrieved FROG spectrum [Figs. 6.4(e) and 6.5(e)] and is free of cw break-throughs, further shown in a logarithmic scale in Figs. 6.4(f) and 6.5(f). The "128 fs result" is close to transformlimited with a spectral bandwidth of $9.48 \mathrm{~nm}$ corresponding to a timebandwidth product of 0.345 , being 1.09 times the value for a sech ${ }^{2}$ pulse shape. The $17.5 \mathrm{~nm}$ broad spectrum of the " $96 \mathrm{fs}$ result" corresponds to a 

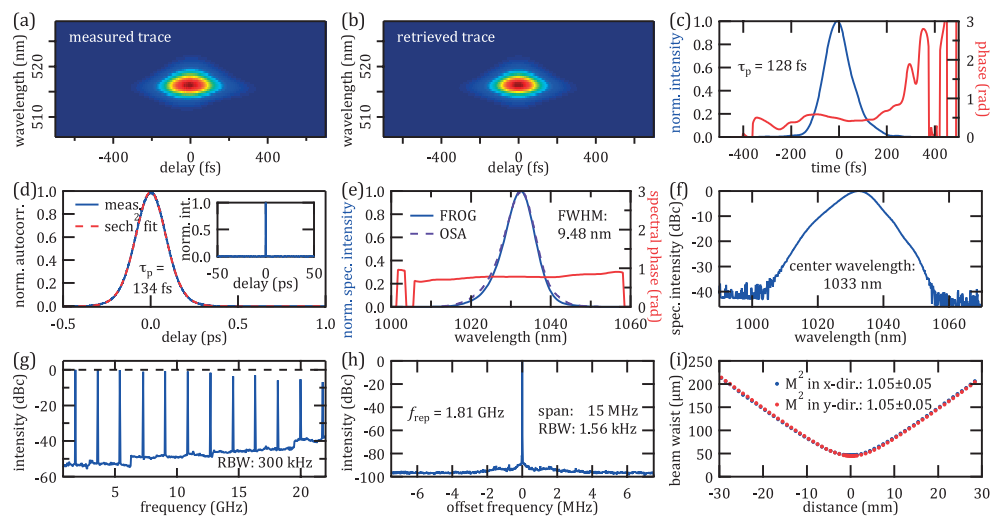

Figure 6.4: "128 fs result": modelocking characterization of the $128 \mathrm{fs}$ pulses at $80 \mathrm{~mW}$ average output power, a peak power of $303 \mathrm{~W}$, and a pulse repetition rate of $1.81 \mathrm{GHz}$. (a) Measured SHG-FROG spectrogram. (b) Retrieved FROG spectrogram (error: 0.0019). (c) Retrieved temporal intensity profile and temporal phase. (d) Intensity autocorrelation with corresponding $\mathrm{sech}^{2}$ fit (inset: long-span autocorrelation without any satellite pulses).(e) Retrieved spectrum and spectral phase overlaid with the measured optical spectrum with a FWHM bandwidth of $9.48 \mathrm{~nm}$. The time-bandwidth product is 1.09 times the ideal value for a sech ${ }^{2}$ pulse shape, i.e., very close to perfect bandwidth-limited pulse duration. (f) Optical spectrum in logarithmic scale centered around $1033 \mathrm{~nm}$, measured with a RBW of $0.1 \mathrm{~nm}$. (g) Long-span microwave spectrum with a RBW of $300 \mathrm{kHz}$. (h) $15 \mathrm{MHz}$ microwave spectrum at $1.81 \mathrm{GHz}$, measured with a RBW of $1.56 \mathrm{kHz}$. (i) Gaussian beam profile confirmed with a measured $\mathrm{m}^{2}<1.05 \pm 0.05$ in two orthogonal directions.

time-bandwidth product of 0.472 , i.e., 1.47 times the ideal value for a sech ${ }^{2}$ pulse shape, and better external pulse compression could potentially support pulses as short as $65 \mathrm{fs}$.

The temporal laser intensity is measured with a fast photo-diode (New Focus, Model 1454), amplified with a broadband and low noise preamplifier (Agilent 87405C) and detected with a microwave spectrum analyzer (Agilent 8565EC, HP 8592L). As discussed in [11], we show two microwave spectra in Figs. 6.4(g) and 6.5(g) and Figs. 6.4(g) and 6.5(g) to further confirm clean fundamental modelocking.

Figures $6.4(\mathrm{~g})$ and $6.5(\mathrm{~g})$ show a scan with high resolution band-width (RBW) of $300 \mathrm{kHz}$ from DC to $22 \mathrm{GHz}$ with a high SNR of $\approx 50 \mathrm{~dB}$. All higher harmonics of the repetition rate exist and are constant, apart from 

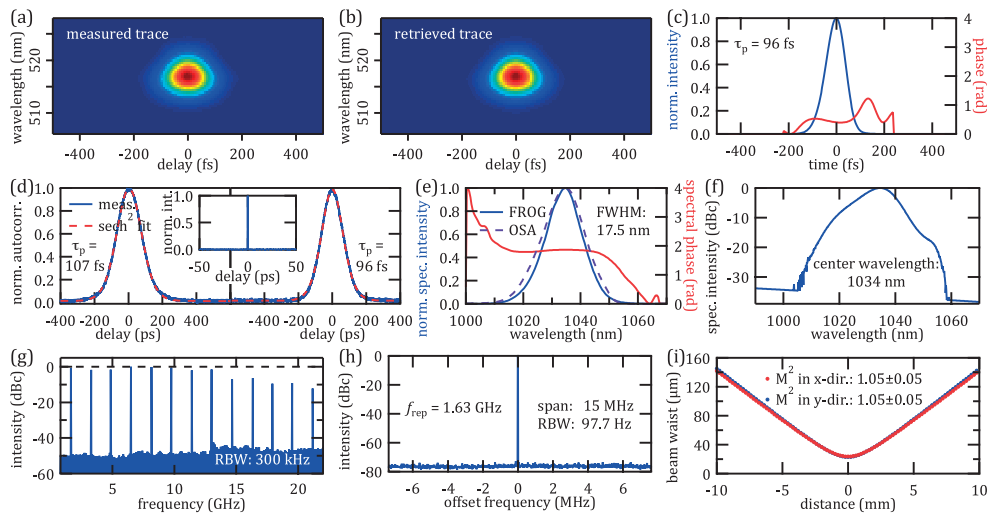

Figure 6.5: "96 fs result": modelocking characterization of the 96-fs pulses at $100 \mathrm{~mW}$ average output power, a peak power of $560 \mathrm{~W}$ and a pulse repetition rate of $1.63 \mathrm{GHz}$. (a) Measured SHG-FROG spectrogram. (b) Retrieved FROG spectrogram (error: 0.0004). (c) Retrieved temporal intensity profile and temporal phase. (d) Intensity autocorrelations with corresponding sech ${ }^{2}$ fits of the $107 \mathrm{fs}$ and $96 \mathrm{fs}$ pulses (inset: long-span autocorrelation without any satellite pulses). (e) Retrieved spectrum and spectral phase overlaid with the measured optical spectrum with a FWHM bandwidth of $17.5 \mathrm{~nm}$. The time-bandwidth product is 1.47 times the ideal value for a sech ${ }^{2}$ pulse shape, i.e., with better dispersion compensation, we could obtain even shorter pulses.(f) Optical spectrum in logarithmic scale centered around $1034 \mathrm{~nm}$, measured with a RBW of $0.1 \mathrm{~nm}$. (g) Long-span microwave spectrum with a RBW of $300 \mathrm{kHz}$. (h) $15 \mathrm{MHz}$ microwave spectrum at $1.63 \mathrm{GHz}$, measured with a RBW of $97.7 \mathrm{~Hz}$. (i) Gaussian beam profile confirmed with a measured $\mathrm{m}^{2}<1.05$ \pm 0.05 in two orthogonal directions.

a small drop in intensity of the higher harmonics due to the limited bandwidth of the $18 \mathrm{GHz}$ preamplifier. Figures 6.4(h) and 6.5(h) zoom into the first harmonic with a higher RBW over an offset frequency span of $\pm 7.5 \mathrm{MHz}$ using a highly linear photodiode (DSC30S-HLPD; Discovery Semiconductors, Inc.) and a signal source analyzer (Agilent E5052B). Even with the high SNR of $\approx 80 \mathrm{~dB}(\approx 90 \mathrm{~dB})$, no line broadening and no additional side peaks become visible. Excellent beam quality was confirmed with measured $\mathrm{M}^{2}$ values of less than $1.05 \pm 0.05$ in two orthogonal directions [Figs. 6.4(g) and 6.5(g)]. 


\subsubsection{Gain characterization}

Here we present both a spectrally resolved measurement of the small signal gain and a measurement of the gain saturation as described by Mangold et al. [37].

\section{Spectral gain measurements}

For the spectral gain characterization, we measured the spectrally resolved small signal reflectivity of a diode-pumped VECSEL. The probe laser is a tunable cw Ti:sapphire laser that is focused tightly to obtain a sub-20- $\mu \mathrm{m}$ diameter beam waist on the surface of the VECSEL chip. The VECSEL is optically pumped by a cw $808 \mathrm{~nm}$ multimode diode laser (Lumics) with an elliptical $122 \mu \mathrm{m} \times 172 \mu \mathrm{m}$ full width at half-maximum (FWHM) spot. The average pump intensity $I_{\mathrm{p}}$ of the probe spot can be varied from 0 to $57 \mathrm{~kW} / \mathrm{cm}^{2}$. Without any pump power, the VECSEL absorbs the probe laser for photon energies exceeding the bandgap energy [Fig. 6.6(a)]. With a pump intensity of approximately $10 \mathrm{~kW} / \mathrm{cm}^{2}$, the laser is pumped into transparency. For increasing pump power, the small signal gain increases, the spectrum becomes broader, and the peak wavelength experiences a redshift due to the elevated temperature in the gain quantum wells.

A shift in the peak wavelength is also visible in Fig. 6.6(b), which shows the small signal gain for different heat-sink temperatures at a constant pump intensity of $57 \mathrm{~kW} / \mathrm{cm}^{2}$. By lowering the heat-sink temperature, the small signal gain increases slightly, and the peak wavelength experiences a blueshift in the range of $0.32 \mathrm{~nm} / \mathrm{K}$, in agreement with the intrinsic temperature dependence of the InGaAs quantum well gain [1]. The maximum small signal gain of $3.17 \%$ is achieved at $57 \mathrm{~kW} / \mathrm{cm}^{2}$ pump in-tensity and a heat-sink temperature of $-5^{\circ} \mathrm{C}$. The small signal gain is lower than the values measured in [37], which can be explained by the reduced field intensity enhancement factor.

The VECSEL chip is heated by the absorbed pump light from the front side and cooled from the backside, creating a temperature gradient normal to the chip surface. This temperature gradient in the active region leads to different temperatures of the individual quantum wells, which shifts the corresponding emission wavelength and thereby broadens the overall accessible macroscopic gain. This effect becomes even stronger with in- 

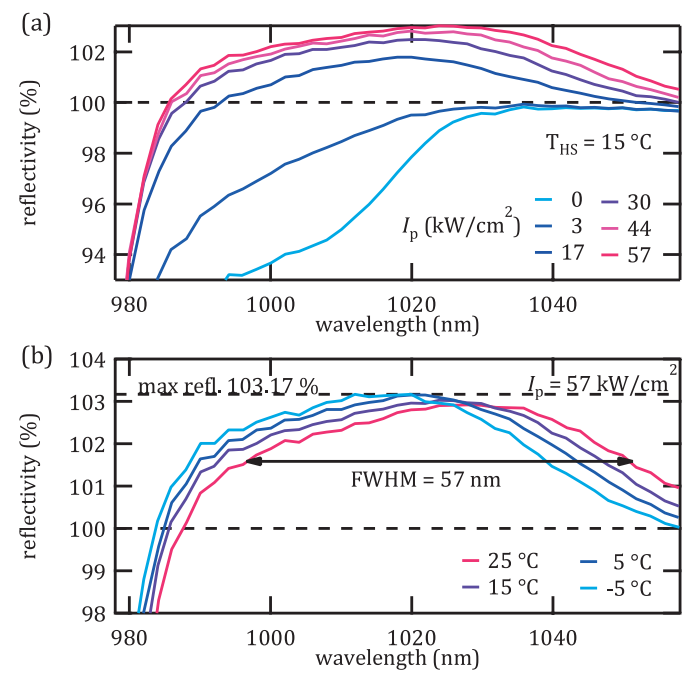

Figure 6.6: Spectral gain measurements. (a) Spectrally resolved reflectivity for different pump intensities at a constant heat-sink temperature of $15^{\circ} \mathrm{C}$. (b) Spectrally resolved reflectivity for different heat-sink temperatures at a constant pump intensity of $I_{\mathrm{p}}=57 \mathrm{~kW} / \mathrm{cm}^{2}$.

creased pump intensities and reduced heat-sink temperatures. The short wavelength limit of the gain shown in Fig. 6.6, is defined by the reflectivity bandwidth of the underlying AlAs/GaAs DBR (full bandwidth of about $110 \mathrm{~nm}$ ), resulting in a gain bandwidth of approximately 50-57 nm. This corresponds to a small signal gain, which is 2-3 times broader than the quantum well photo-luminescence measured by Wang et al. [7].

\section{Gain saturation measurements}

For the gain saturation characterization, we measured the fluencedependent reflectivity of the diode-pumped VECSEL. The probe laser is a tunable modelocked Ti:sapphire laser generating $140 \mathrm{fs}$ pulses. The probe light is focused to a $29.6 \mu \mathrm{m}$ diameter spot. The pump arrangement is the same as the one used for the spectral gain measurement, resulting in a maximum pump intensity of $46.5 \mathrm{~kW} / \mathrm{cm}^{2}$. The dependence of the reflectivity on the probe fluence is measured for different probe wavelengths, 
pump fluences, and heat-sink temperatures. The probe fluence is varied over 3 orders of magnitude up to $1200 \mu \mathrm{J} / \mathrm{cm}^{2}$ for probing wave-lengths of 1010, 1020, 1030, and $1040 \mathrm{~nm}$. The pump intensity is varied between 0 and $46.5 \mathrm{~kW} / \mathrm{cm}^{2}$, while the heat-sink temperature is kept at $-5^{\circ} \mathrm{C}, 5^{\circ} \mathrm{C}$, $15^{\circ} \mathrm{C}$, or $25^{\circ} \mathrm{C}$.

The nonlinear reflectivity [Fig. 6.7(a)] is analyzed with a macroscopic model for the gain saturation analogous to the saturable absorption of a SESAM. The characteristic parameters are the small signal reflectivity, the gain saturation fluence, the nonsaturable losses, and the induced absorption. The detailed description of the model can be found in [37].

To study the measured gain saturation parameters, the concept of the equivalent wavelength is introduced, which allows for a matching of measurement series taken at different probing wavelengths and temperatures. As visible in Fig. 6.6(b), the quantum well emission is blueshifted by approximately $3.2 \mathrm{~nm}$ for a heat-sink temperature reduction of $10^{\circ} \mathrm{C}$. To account for this blueshift, the probing wavelength of $1030 \mathrm{~nm}$ at a heat-sink temperature of $15^{\circ} \mathrm{C}$ corresponds to an equivalent probing wavelength of $1033.2 \mathrm{~nm}$ at a heat-sink temperature of $25^{\circ} \mathrm{C}$, for example. Following this concept, the equivalent probing wavelength is calculated for all temperatures lower than $25^{\circ} \mathrm{C}$.

The saturation fluence varies between 30 and $50 \mu \mathrm{J} / \mathrm{cm}^{2}$ and has a slight dip around $1030 \mathrm{~nm}$ [Fig. 6.7(b)]. The saturation fluence is comparable to that reported in [37], although the saturation fluence is expected to scale inversely proportional to the field intensity enhancement factor, and, therefore, we have expected higher saturation fluences. Figure 6.7(c) shows the small signal reflectivity of the probing pulse with an $11 \mathrm{~nm}$ wide spectrum analogous to the reflectivity spectrum [Fig. 6.6(b)] of a single frequency $\mathrm{cw}$ probe. The small signal reflectivity has a maximum around $1030 \mathrm{~nm}$ and is slightly reduced for lower heat-sink temperatures. The induced absorption shows a weak temperature dependence and has a maximum of $17.6 \mathrm{~mJ} / \mathrm{cm}^{2}$ at a probing wavelength of $1030 \mathrm{~nm}$, as shown in Fig. 6.7(d). 

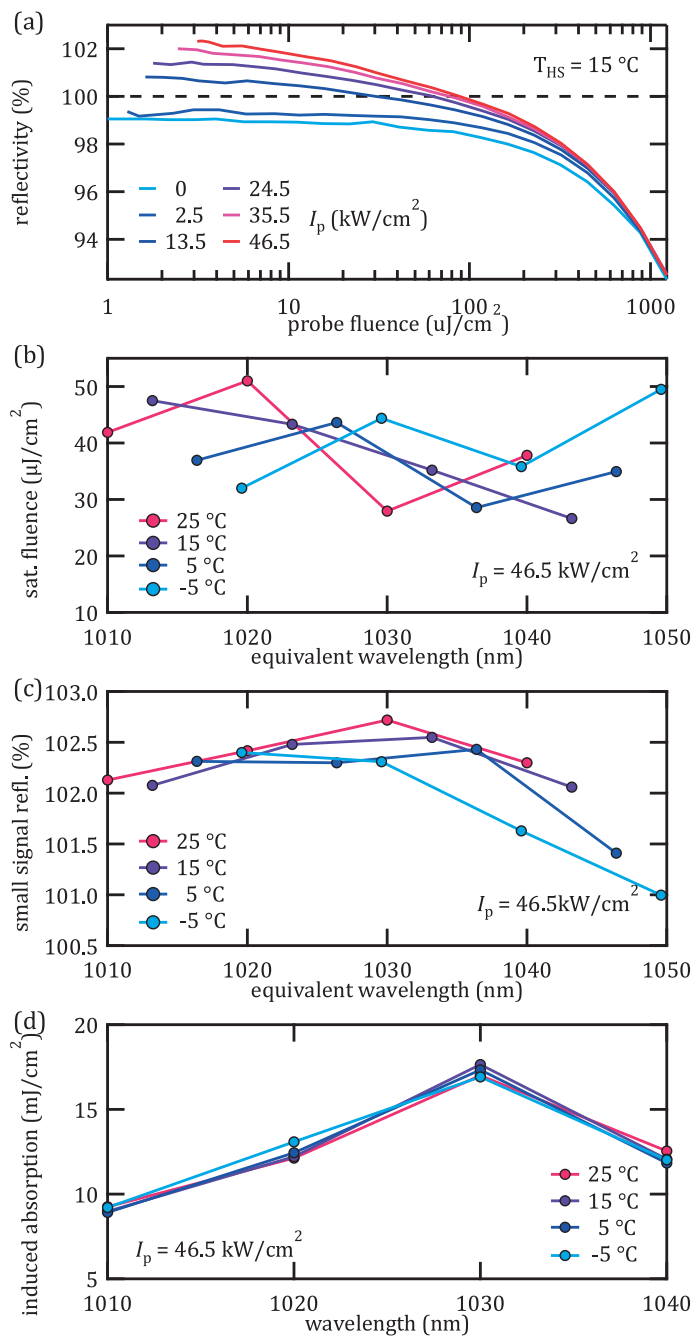

Figure 6.7: Gain saturation measurements. (a) Fluence-dependent reflectivity for different pump intensities at a heat-sink temperature of $15^{\circ} \mathrm{C}$.(b) Saturation fluence as a function of heat-sink temperature and equivalent wavelength. (c) Small signal reflectivity as a function of heat-sink temperature and equivalent wavelength. The equivalent wavelength accounts for the temperature-induced wavelength shift of the gain peak wavelength for matching of measurement series. See text for a more detailed definition of equivalent wavelength. (d) Induced absorption as a function of wavelength and heat-sink temperature. 


\subsubsection{Pulse formation simulation}

We used our pulse formation simulations as described in [34]. This simulation is based on macroscopic input parameters summarized in Table 6.1. We used the same linewidth enhancement factors, the same additional cavity losses, and the same gain recovery time as discussed before in [34]. The input parameters of the VECSEL gain chip are chosen in the middle of the measured range of 50-57 nm (FWHM) for the gain bandwidth, 30-50 $\mathrm{\mu J} / \mathrm{cm}^{2}$ for the gain saturation fluence, and $2.5 \%-3.17 \%$ for the small signal gain. More elaborated simulations starting from the fully microscopic many-body theory by Kilen et al. [41] indicated the limitations of macroscopic models for pulse durations below some hundreds of femtoseconds. Nevertheless, so far our macroscopic models have shown good agreement with our experimental results.

Our simulation shows that the combination of the gain bandwidth [Fig. 6.6(b)] and the gain saturation fluence [Fig. 6.7(b)] defines a range of stable modelocking configurations and, so, the minimum possible pulse duration. As expected, the small signal gain [Fig. 6.6(b)] and the losses mainly determine the output power. The values for dispersion per cavity round-trip used for the simulation are not straightforward because, for example, the dispersion of the VECSEL [Fig. 6.3(b)] was not measured under lasing operation. Furthermore, the modelocking simulations are very sensitive to dispersion, with increasing sensitivity for increasing gain bandwidth. The cavity round-trip dispersion fitting the "96 fs result" is $-12 \mathrm{fs}^{2}$ of GDD and $-500 \mathrm{fs}^{3}$ of third-order dispersion (TOD), and these values are $12 \mathrm{fs}^{2}$ of GDD and $620 \mathrm{fs}^{3}$ of TOD for the "128 fs result." These dispersion values are shown in Fig. 6.3(b) and are in reasonable agreement with the dispersion of the VECSEL, which is expected to give the main contribution.

The pulse formation simulation for the " $128 \mathrm{fs}$ input parameters" (Table 6.1,Fig.8) not only agrees with the pulse duration of $128 \mathrm{fs}$, but also with the spectral bandwidth of $9.5 \mathrm{~nm}$ (measured $9.48 \mathrm{~nm}$ ) and the average output power of $78 \mathrm{~mW}$ (measured $80 \mathrm{~mW}$ ). The $96 \mathrm{fs}$ input parameters (Table 6.1) with the dispersion adapted to fit the compressed 96 fs pulses (Table 6.1: $-12 \mathrm{fs}^{2}$ and $-500 \mathrm{fs}^{3}$ ) results in negatively chirped $130 \mathrm{fs}$ output pulses (i.e., not the measured $107 \mathrm{fs}$ ), which can be compressed with 

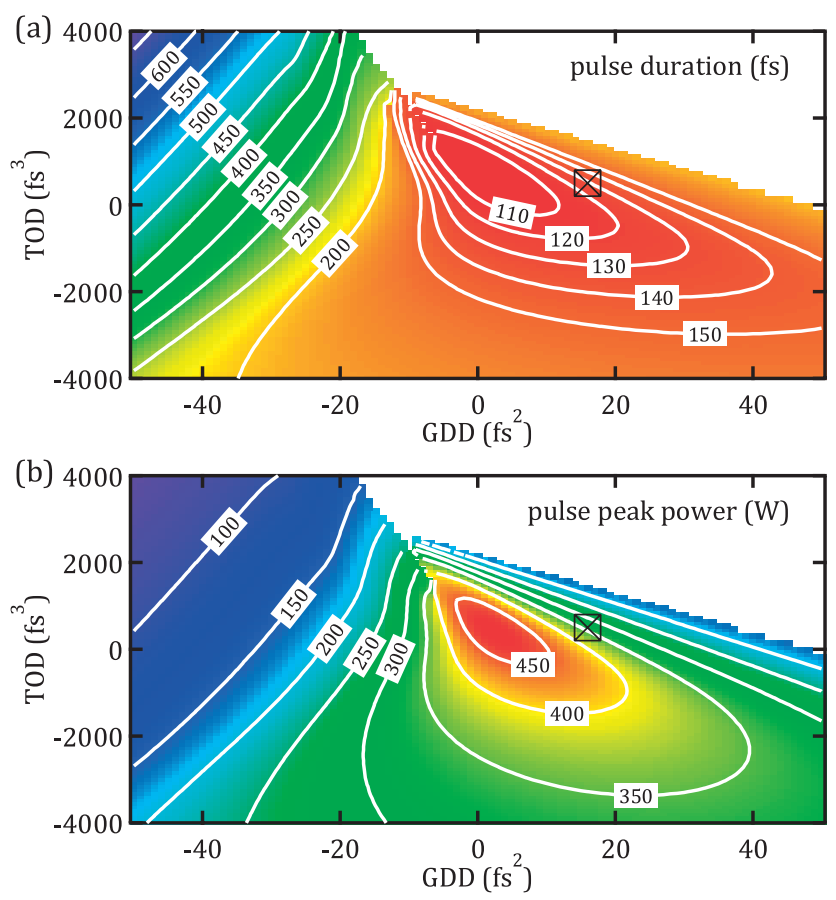

Figure 6.8: Numerical pulse formation simulations using the $128 \mathrm{fs}$ input parameters from Table 6.1. Cavity round-trip dispersion management for both (a) a shorter pulse duration and (b) a higher peak power as functions of second-order dispersion (i.e., GDD) and TOD is shown. The best performance is obtained in the red shaded area, and the "128 fs result" is marked with a black cross.

$1350 \mathrm{fs}^{2}$ positive GDD to $96 \mathrm{fs}$, a spectral bandwidth of $17.5 \mathrm{~nm}$, and an average output power of $104 \mathrm{~mW}$. With a slightly different cavity round-trip GDD of $-4 \mathrm{fs}^{2}$ and TOD of- $600 \mathrm{fs}^{3}$, we can fit the $107 \mathrm{fs}$ output pulses: the simulation results in negatively chirped 108 fs output pulses, which can be com-pressed with $1350 \mathrm{fs}^{2}$ positive GDD to $86 \mathrm{fs}$ (i.e., not the measured $96 \mathrm{fs})$, a spectral bandwidth of $17.4 \mathrm{~nm}$, and an average output power of $100 \mathrm{~mW}$ (measured: 107 fs, 96 fs, $17.5 \mathrm{~nm}, 100 \mathrm{~mW}$ ). Higher order dispersion of the ZnSe plate has not been taken into account. The agreement is no longer perfect, but the simulation confirms the $100 \mathrm{fs}$ pulse duration with initially negatively chirped output pulses. At this point, we did 
not include the nonlinear induced absorption in the SESAM and VECSEL even though this becomes more important with shorter pulses.

Using the $128 \mathrm{fs}$ input parameters (Table 6.1), we further explored the sensitivity of the cavity round-trip dispersion taking into account both the second-order dispersion (GDD) and the TOD. Different values of TOD are applied in addition to the GDD, centered around the lasing frequency (Fig. 6.8), with the cross indicating the dispersion values used for fitting the "128 fs result". The shortest pulses (101 fs) are achieved with $2 \mathrm{fs}^{2}$ GDD and $560 \mathrm{fs}^{3}$ TOD. The highest pulse peak power is $480 \mathrm{~W}$ and is obtained with $3 \mathrm{fs}^{2}$ GDD and $400 \mathrm{fs}^{3}$ TOD. Similar sensitivity is observed with the $96 \mathrm{fs}$ input parameters, but is not shown here.

\subsubsection{Performance scaling}

From our simulations as presented in Section 6.1 .5 we can conclude that the shortest pulse duration is mainly determined by the gain bandwidth, the gain saturation fluence, and the second- and third-order dispersion of the VECSEL. Figure 6.8 summarizes the general sensitivity on secondand third-order dispersion for both shorter pulse durations [red area in Fig. 6.8(a)] and higher pulse peak powers [red area in Fig. 6.8(b)]. Slightly positive TOD is needed for the shortest pulses. Too much positive TOD, how-ever, causes instabilities, while negative TOD results in stable modelocking with slowly increasing pulse duration and decreasing peak power (Fig. 6.8). Negative GDD should be avoided, as the pulse duration increases rapidly into the picosecond regime, while for positive GDD, the pulse duration increases more slowly. Thus dispersion management becomes even more critical for broad-band gain. Most recently, this has also been confirmed by Head et al. [35]. Control of the GDD and TOD of a VECSEL is challenging, as they are very sensitive to growth and fabrication uncertainties. Even growth errors in the 1\% range can significantly change the wavelength-dependent dispersion. The simulation and the modelocking results confirm that a VECSEL gain bandwidth of 50$57 \mathrm{~nm}$ (FWHM) is sufficient for the generation of sub-100-fs pulses.

In the future we will focus our efforts toward even higher average output power and higher optical-to-optical efficiency of sub-100-fs SESAM modelocked VECSELs. The optimized broadband gain has been the 
key enabler for these new milestone results and has been achieved with flat field intensity enhancement, broadband dispersion control, and highquality epitaxial growth. Higher gain saturation fluence would further increase the output power and simultaneously shorten the pulse duration. The measured saturation fluences [Fig. 6.7(b)] have been lower than expected in comparison to [37] with the corresponding field intensity enhancement factors taken into account. However, we changed the lasing wavelength (1030 nm instead of $960 \mathrm{~nm}$ ) and the epitaxial growth (MOVPE instead of MBE), which makes a direct comparison more difficult.

\subsubsection{Conclusion}

In conclusion, we have presented a significant improvement of SESAM modelocked VECSELs with both shorter pulse durations and higher average power with $96 \mathrm{fs}$ pulses and $560 \mathrm{~W}$ pulse peak power. We performed a detailed characterization of both the VECSEL and the SESAM parameters, which were used as input parameters in our simulations (Table 6.1). We obtained good agreement between experiment and theory. Both second- and third-order dispersion management is required for short pulse durations and transform-limited pulses. The " $128 \mathrm{fs}$ result" is very close to transform limited with a time-bandwidth product of 1.09 times the ideal value. The 96 fs pulses were obtained by additional external dispersion compensation with a single path through a 2-mm-thick zinc selenide plate and are still not transform limited, with a time-bandwidth product of 1.47 times the ideal value of a sech ${ }^{2}$ pulse shape. The bandwidth could in principle support transform-limited pulses as short as $65 \mathrm{fs}$. We have explained the sensitivity and importance of dispersion management and outlined design guidelines for future improvements in output power. With these record modelocking results, we have demonstrated that high-power $100 \mathrm{fs}$ pulses are possible from optically pumped ultrafast VECSELs. This performance will enable octave-spanning supercontinuum generation without any additional external pulse amplifications based on recent progress with $\mathrm{Si}_{3} \mathrm{~N}_{4}$ waveguides $[27,28]$ for supercontinuum generation.

Funding. Swiss Confederation Program Nano-Tera.ch.

Acknowledgement We thank the technology and clean-room facility 
FIRST of ETH Zürich for advanced micro- and nanotechnology. This work was financed by the Swiss Confederation Program Nano-Tera.ch, which was scientifically evaluated by the Swiss National Science Foundation (SNSF).

\subsubsection{References}

[1] U. Keller and A. C. Tropper, "Passively modelocked surface-emitting semiconductor lasers," Phys. Rep. 429,67-120 (2006).

[2] U. Keller, K. J. Weingarten, F. X. Kärtner, D. Kopf, B. Braun, I. D. Jung, R. Fluck, C. Hönninger, N. Matuschek, and J. Aus der Au, "Semiconductor saturable absorber mirrors (SESAMs) for femtosecond to nanosecond pulse generation in solid-state lasers," IEEE J. Sel. Top. Quantum Electron. 2, 435-453 (1996).

[3] B. W. Tilma, M. Mangold, C. A. Zaugg, S. M. Link, D. Waldburger, A. Klenner, A. S. Mayer, E. Gini, M. Golling, and U. Keller, "Recent advances in ultrafast semiconductor disk lasers," Light Sci. Appl. 4, e310 (2015).

[4] M. Kuznetsov, F. Hakimi, R. Sprague, and A. Mooradian, "Highpower ( $>0.5-\mathrm{W} \mathrm{CW}$ ) diode-pumped vertical-external-cavity surfaceemitting semiconductor lasers with circular TEM00 beams," IEEE Photon. Technol. Lett. 9, 1063-1065 (1997).

[5] S. H. Park, J. Kim, H. Jeon, T. Sakong, S. N. Lee, S. Chae, Y. Park, C. H. Jeong, G. Y. Yeom, and Y. H. Cho, "Room-temperature GaN

[6] M. Rahim, M. Arnold, F. Felder, K. Behfar, and H. Zogg, "Midinfrared lead-chalcogenide vertical external cavity surface emitting laser with 5 mm wavelength," Appl. Phys. Lett. 91, 151102 (2007).

[7] T. L. Wang, Y. Kaneda, J. M. Yarborough, J. Hader, J. V. Moloney, A. Chernikov, S. Chatterjee, S. W. Koch, B. Kunert, and W. Stolz, "Highpower optically pumped semiconductor laser at $1040 \mathrm{~nm}$," IEEE Photon. Technol. Lett. 22, 661-663 (2010).

[8] B. Rudin, A. Rutz, M. Hoffmann, D. J. H. C. Maas, A.-R. Bellancourt, E. Gini, T. Südmeyer, and U. Keller, “Highly efficient optically 
pumped vertical emitting semiconductor laser with more than $20 \mathrm{~W}$ average output power in a fundamental transverse mode," Opt. Lett. 33, 2719-2721 (2008).

[9] A. Laurain, C. Mart, J. Hader, J. V. Moloney, B. Kunert, and W. Stolz, " $15 \mathrm{~W}$ single frequency optically pumped semiconductor laser with sub-megahertz linewidth," IEEE Photon. Technol. Lett. 26, 131-133 (2014).

[10] B. Heinen, T. L. Wang, M. Sparenberg, A. Weber, B. Kunert, J. Hader, S. W. Koch, J. V. Moloney, M. Koch, and W. Stolz, "106 W continuouswave output power from vertical-external-cavity surface-emitting laser," Electron. Lett. 48, 516-517 (2012).

[11] M. Mangold, M. Golling, E. Gini, B. W. Tilma, and U. Keller, "Sub300-femtosecond operation from a MIXSEL," Opt. Express 23, 2204322059 (2015).

[12] I. Kilen, J. Hader, J. V. Moloney, and S. W. Koch, "Ultrafast nonequilibrium carrier dynamics in semiconductor laser mode locking," Optica 1, 192-197 (2014).

[13] P. Klopp, U. Griebner, M. Zorn, and M. Weyers, "Pulse repetition rate up to $92 \mathrm{GHz}$ or pulse duration shorter than $110 \mathrm{fs}$ from a modelocked semiconductor disk laser," Appl. Phys. Lett. 98, 071103 (2011).

[14] A. H. Quarterman, K. G. Wilcox, V. Apostolopoulos, Z. Mihoubi, S. P. Elsmere, I. Farrer, D. A. Ritchie, and A. Tropper, "A passively modelocked external-cavity semiconductor laser emitting 60-fs pulses," Nat. Photonics 3, 729-731 (2009).

[15] K. G. Wilcox, A. C. Tropper, H. E. Beere, D. A. Ritchie, B. Kunert, B. Heinen, and W. Stolz, " $4.35 \mathrm{~kW}$ peak power femtosecond pulse mode-locked VECSEL for supercontinuum generation," Opt. Express 21, 1599-1605 (2013).

[16] C. J. Saraceno, F. Emaury, C. Schriber, M. Hoffmann, M. Golling, T. Südmeyer, and U. Keller, "Ultrafast thin-disk laser with $80 \mu \mathrm{J}$ pulse energy and $242 \mathrm{~W}$ of average power," Opt. Lett. 39,9-12 (2014). 
[17] C. A. Zaugg, A. Klenner, O. D. Sieber, M. Golling, B. W. Tilma, and U. Keller, "Sub-100 MHz passively modelocked VECSEL," in Conference on Lasers and Electro Optics (CLEO) (Optical Society of America, 2013), paper CW1G.6.

[18] M. Butkus, E. A. Viktorov, T. Erneux, C. J. Hamilton, G. Maker, G. P. A. Malcolm, and E. U. Rafailov, “85.7 MHz repetition rate modelocked semiconductor disk laser: fundamental and soliton bound states," Opt. Express 21, 25526-25531 (2013).

[19] M. Hoffmann, Y. Barbarin, D. J. H. C. Maas, M. Golling, I. L. Krestnikov, S. S. Mikhrin, A. R. Kovsh, T. Südmeyer, and U. Keller, "Modelocked quantum dot vertical external cavity surface emitting laser," Appl. Phys. B 93, 733-736 (2008).

[20] M. Hoffmann, O. D. Sieber, V. J. Wittwer, I. L. Krestnikov, D. A. Livshits, Y. Barbarin, T. Südmeyer, and U. Keller, "Femtosecond highpower quantum dot vertical external cavity surface emitting laser," Opt. Express 19, 8108-8116 (2011).

[21] R. Bek, G. Kersteen, H. Kahle, T. Schwarzbäck, M. Jetter, and P. Michler, "All quantum dot mode-locked semiconductor disk laser emitting at 655 nm," Appl. Phys. Lett. 105, 082107 (2014).

[22] R. Aviles-Espinosa, G. Filippidis, C. Hamilton, G. Malcolm, K. J. Weingarten, T. Südmeyer, Y. Barbarin, U. Keller, S. I. C. O. Santos, D. Artigas, and P. Loza-Alvarez, "Compact ultrafast semiconductor disk laser: targeting GFP based nonlinear applications in living organisms," Biomed. Opt. Express 2, 739-747 (2011).

[23] C. A. Zaugg, A. Klenner, M. Mangold, A. S. Mayer, S. M. Link, F. Emaury, M. Golling, E. Gini, C. J. Saraceno, B. W. Tilma, and U. Keller, "Gigahertz self-referenceable frequency comb from a semiconductor disk laser," Opt. Express 22, 16445-16455 (2014).

[24] A. Klenner, S. Schilt, T. Südmeyer, and U. Keller, "Gigahertz frequency comb from a diode-pumped solid-state laser," Opt. Express 22, 31008-31019 (2014).

[25] A. H. Quarterman, L. E. Hooper, P. J. Mosley, and K. G. Wilcox, "Gigahertz pulse source by compression of mode-locked VECSEL 
pulses coherently broadened in the normal dispersion regime," Opt. Express 22, 12096-12101 (2014).

[26] H. R. Telle, G. Steinmeyer, A. E. Dunlop, J. Stenger, D. H. Sutter, and U. Keller, "Carrier-envelope offset phase control: a novel concept for absolute optical frequency measurement and ultrashort pulse generation," Appl. Phys. B 69, 327-332 (1999).

[27] A. Klenner, A. S. Mayer, A. R. Johnson, K. Luke, M. R. E. Lamont, Y. Okawachi, M. Lipson, A. L. Gaeta, and U. Keller, “Gigahertz frequency comb offset stabilization based on supercontinuum generation in silicon nitride waveguides," Opt. Express 24, 11043-11053 (2016).

[28] A. S. Mayer, A. Klenner, A. R. Johnson, K. Luke, M. R. E. Lamont, Y. Okawachi, M. Lipson, A. L. Gaeta, and U. Keller, “Frequency comb offset detection using supercontinuum generation in silicon nitride wave-guides," Opt. Express 23, 15440-15451 (2015).

[29] C. Hönninger, R. Paschotta, F. Morier-Genoud, M. Moser, and U. Keller, "Q-switching stability limits of continuous-wave passive mode locking," J. Opt. Soc. Am. B 16,46-56 (1999).

[30] A. Klenner and U. Keller, "All-optical Q-switching limiter for highpower gigahertz modelocked diode-pumped solid-state lasers," Opt. Express 23, 8532-8544 (2015).

[31] D. Hillerkuss, R. Schmogrow, T. Schellinger, M. Jordan, M. Winter, G. Huber, T. Vallaitis, R. Bonk, P. Kleinow, F. Frey, M. Roeger, S. Koenig, A. Ludwig, A. Marculescu, J. Li, M. Hoh, M. Dreschmann, J. Meyer, S. Ben Ezra, N. Narkiss, B. Nebendahl, F. Parmigiani, P. Petropoulos, B. Resan, A. Oehler, K. Weingarten, T. Ellermeyer, J. Lutz, M. Moeller, M. Huebner, J. Becker, C. Koos, W. Freude, and J. Leuthold, " 26 Tbit s-1 line-rate super-channel transmission utilizing all-optical fast Fourier transform processing," Nat. Photonics 5, 364371 (2011).

[32] M. Mangold, C. A. Zaugg, S. M. Link, M. Golling, B. W. Tilma, and U. Keller, "Pulse repetition rate scaling from 5 to $100 \mathrm{GHz}$ with a high-power semiconductor disk laser," Opt. Express 22, 6099-6107 
(2014).

[33] D. Waldburger, C. G. E. Alfieri, S. M. Link, E. Gini, M. Golling, M. Mangold, B. W. Tilma, and U. Keller, "Pulse shortening of an ultrafast VECSEL," Proc. SPIE 9734, 973409 (2016).

[34] O. D. Sieber, M. Hoffmann, V. J. Wittwer, M. Mangold, M. Golling, B. W. Tilma, T. Südmeyer, and U. Keller, "Experimentally verified pulse formation model for high-power femtosecond VECSELs," Appl. Phys. B 113, 133-145 (2013).

[35] C. R. Head, A. Hein, A. P. Turnbull, M. Polanik, E. A. Shaw, T. Chen Sverre, P. Unger, and A. C. Tropper, "High-order dispersion in sub200- fs pulsed VECSELs," Proc. SPIE 9734, 973408 (2016).

[36] D. J. H. C. Maas, B. Rudin, A.-R. Bellancourt, D. Iwaniuk, S. V. Marchese, T. Südmeyer, and U. Keller, "High precision optical characterization of semiconductor saturable absorber mirrors," Opt. Express 16, 7571-7579 (2008).

[37] M. Mangold, V. J. Wittwer, O. D. Sieber, M. Hoffmann, I. L. Krestnikov, D. A. Livshits, M. Golling, T. Südmeyer, and U. Keller, “VECSEL gain characterization," Opt. Express 20, 4136-4148 (2012).

[38] G. J. Spühler, K. J. Weingarten, R. Grange, L. Krainer, M. Haiml, V. Liverini, M. Golling, S. Schon, and U. Keller, "Semiconductor saturable absorber mirror structures with low saturation fluence," Appl. Phys. B 81, 27-32 (2005).

[39] S. Hoogland, S. Dhanjal, A. C. Tropper, S. J. Roberts, R. Häring, R. Paschotta, and U. Keller, "Passively mode-locked diode-pumped surface-emitting semiconductor laser," IEEE Photon. Technol. Lett. $12,1135-1137$ (2000).

[40] A. Gosteva, M. Haiml, R. Paschotta, and U. Keller, "Noise-related resolution limit of dispersion measurements with white-light interferometers," J. Opt. Soc. Am. B 22, 1868-1874 (2005).

[41] I. Kilen, S. W. Koch, J. Hader, and J. V. Moloney, "Fully microscopic modeling of mode locking in microcavity lasers," J. Opt. Soc. Am. B 33,75-80 (2016). 



\section{Chapter 7}

\section{Colliding pulse modelocked VECSELs}

The external cavity of SDLs creates the possibility for intracavity elements and to build different kinds of cavities around the semiconductor chip. In case of the MIXSEL the intracavity birefringent crystal enabled dual-comb modelocking. For VECSELs, besides the standard V-shaped cavity, also ring cavities are possible, enabling colliding pulse modelocking (CPM) [51]. The recently demonstrated performance of a CPM VECSEL is with $128 \mathrm{fs}$ and $90 \mathrm{~mW}$ average output power [52] comparable to state of the art VECSELs in a V-shaped cavity (see last chapter). The ring cavity adds additional complexity compared to the V-shaped cavity, however, instead of one output beam, two beams are coupled out from the two counter propagating pulse trains inside the ring cavity. A coherent beam combination of these two beams could therefore double the output power, which could be the missing factor in terms of power for supercontinuum generation with SDLs. Coherent beam combining requires that the CEO frequency as well as the pulse repetition frequencies of both beams are locked to each other. Similar concepts and measurement techniques as used for the pulse repetition rate stabilization in chapter 2 and chapter 4 and for the optical linewidth stability measurement in chapter 5 can be applied to determine, wether these two requirements are fulfilled.

The confirmation, that both CEO and pulse repetition frequencies are locked and a first proof-of-principle demonstration of coherent beam combining of a CPM VECSEL are demonstrated in the following Optics Express publication [53]. (C) 2017 Optical Society of America. One print or 
electronic copy may be made for personal use only. Systematic reproduction and distribution, duplication of any material in this paper for a fee or for commercial purposes, or modifications of the content of this paper are prohibited.)

\subsection{Coherent beam combining and noise analysis of a colliding pulse modelocked VECSEL}

Sandro M. Link, Dominik Waldburger, Cesare G. E. Alfieri, Matthias Golling and Ursula Keller

Department of Physics, Institute for Quantum Electronics, ETH Zürich, 8093 Zürich, Switzerland

Optically-pumped SESAM-modelocked semiconductor disk lasers have become interesting ultrafast lasers with gigahertz pulse repetition rates, high average power and adjustable lasing wavelength. It is well established that colliding pulse modelocking (CPM) can generate both shorter pulses and improved stability. These improvements however typically come at the expense of a more complex ring cavity and two output beams. So far similar modelocking results have been obtained with CPM vertical external-cavity surface-emitting lasers (VECSELs) and with SESAM-modelocked VECSELs or modelocked integrated external-cavity surface-emitting lasers (MIXSELs) in a linear cavity. However coherent beam combining of the two output beams of a CPM VECSEL could result in a significantly higher peak power. This is interesting for example for applications in biomedical microscopy and frequency metrology. Here we demonstrate with a more detailed noise analysis that for both output beams of a CPM VECSEL the pulse repetition rates and the carrier envelope offset frequencies are locked to each other. In contrast to standard SESAM-modelocked VECSELs in a linear cavity, we only have been able to actively stabilize the pulse repetition rate of the CPM VECSEL by cavity length control and not by pump-power control. Furthermore, a first coherent beam combining experiment of the two output beams is demonstrated. 


\subsubsection{Introduction}

Colliding pulse modelocking (CPM) was first introduced with passively modelocked dye lasers [1]. The modelocking is based on dynamic gain saturation for which a critical balance between loss and gain saturation opens up a net gain window [2]. Better modelocking stability and shorter pulses were achieved with a ring laser when the distance between the absorber and the gain is about one quarter of the resonator roundtrip length. In this case, the two pulses propagating in opposite direction collide in the saturable absorber and saturate the absorber more strongly. In addition, the time between the pulses in the gain corresponds to half the roundtrip time which allows for the gain to recover to the same value for both pulses. The optimization of the CPM Rhodamine 6G laser resulted in world-record pulse durations of only $27 \mathrm{fs}$ [3]. The CPM technique then has been also applied for semiconductor lasers with a monolithic edge-emitting quantum well laser in a linear cavity [4, 5]. However, the critical cavity stability requirements seem to have prevented a stronger impact of this approach so far. More recently the CPM technique has been used for an optically pumped vertical-external cavity surface-emitting laser (VECSEL) in a ring cavity [6] generating pulses as short as $128 \mathrm{fs}$ with an average power of $90 \mathrm{~mW}$ per output beam at a pulse repetition rate of $3.27 \mathrm{GHz}$ [7].

However, it is not clear that the additional complexity of a ring cavity is justified when we compare CPM VECSELs with standard SESAMmodelocked VECSELs $[8,9]$. Stable modelocking with pulses as short as $96 \mathrm{fs}$ with $100 \mathrm{~mW}$ of output power in a more simple linear cavity has been demonstrated [10]. In addition, CPM is not possible with the more compact approach of a modelocked integrated external-cavity surface-emitting laser (MIXSEL) [11] integrating the saturable absorber and the gain within the same semiconductor wafer. To date with optically pumped MIXSELs pulses as short as $184 \mathrm{fs}$ with $115 \mathrm{~mW}$ average output power have been demonstrated [12]. The MIXSEL also provides a very stable optical frequency comb even without any further active stabilizations: for example a free-running single 2-GHz MIXSEL comb has a comb line spacing variation of only $\approx 2.5 \times 10^{-4}$ integrated over a measurement time of $10 \mathrm{~ms}$ [13]. Such excellent modelocking stability enabled dual comb spectroscopy in water vapor with only one laser cavity and without any further active 
stabilization [14].

A CPM VECSEL has two output beams and so far it has not been demonstrated that these two beams are fully coherent and potentially could be combined to obtain a higher peak power. This could be interesting for applications in biomedical microscopy [15] and supercontinuum generation [16]. Coherent beam combining requires that both the pulse repetition frequencies and the carrier envelope offset (CEO) frequencies [17] are identical for both beams, which has not been demonstrated for CPM VECSELs to date. Here, we analyze the noise behavior of a CPM VECSEL and confirm that indeed, both the pulse repetition frequencies and the CEO frequencies of both beams are intrinsically locked to each other. We coherently combine the two beams in a proof-of-principle second harmonic generation (SHG) experiment.

\subsubsection{Laser setup}

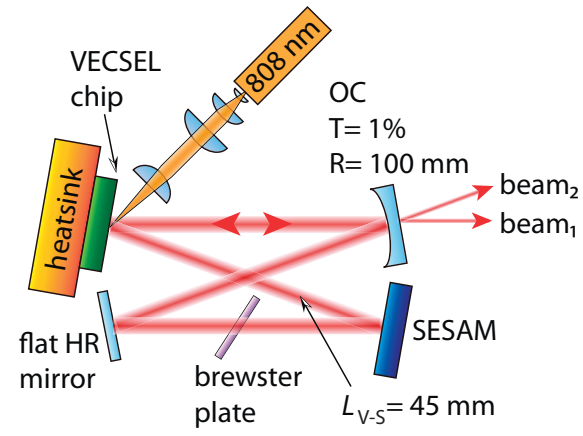

Figure 7.1: Colliding pulse modelocked (CPM) VECSEL. The VECSEL chip is optically pumped by a cw 808-nm diode laser array. The ring cavity enables two pulses propagating in opposite directions. Stable CPM is obtained when the cavity length between the VECSEL chip and the SESAM (LV-S) is a quarter of the total cavity roundtrip length. OC, output coupler; HR, high reflector.

The details of the VECSEL chip used to build the ring cavity can be found in [10]. The cavity comprises the VECSEL chip, the SESAM, a flat high reflectance mirror and an output coupler (OC) with $1 \%$ transmission 
and a radius of curvature of $100 \mathrm{~mm}$ [Fig. 7.1]. The total cavity length is $180 \mathrm{~mm}$. Since there is no direction-selective element in the ring cavity, two pulses can propagate in opposite direction around the ring cavity. The CPM technique introduces a stronger saturation of the saturable absorber based on interference of the two counter-propagating beams. This intrinsically favors the same linear polarization for both output beams which was also observed in our experiment. However, depending on the pump power, we have observed that both beams can simultaneously switch from p- to s-polarization. This was also observed in the linear cavity configuration using the same VECSEL chip. Since part of the experiment is polarization sensitive such as the second harmonic crystal, we introduced an intracavity Brewster plate to fix the polarization. For the distance between the VECSEL chip and the SESAM (LV-S) a quarter of the total cavity length is chosen to enable an equal pumping duration and gain recovery time for both counter-propagating pulses [6].

The VECSEL chip and the SESAM are operated at $20^{\circ} \mathrm{C}$ and $18^{\circ} \mathrm{C}$, respectively. The cavity and pump spot on the gain chip have a beam radius of $177 \mu \mathrm{m}$ and the beam radius on the SESAM is $100 \mu \mathrm{m}$. With a total pump power of $20 \mathrm{~W}$, we achieved $170 \mathrm{~mW}$ average output power per beam. The diagnostics show that both beams have identical modelocking characteristics [Fig. 7.2]. The center wavelength of both beams is $1034.3 \mathrm{~nm}$ with a full width at half maximum (FWHM) of $2.4 \mathrm{~nm}$ [Fig. 7.2(a)]. The pulse duration, measured with a second-harmonic autocorrelation, is for both beams 780 fs [Fig. 7.2(b)] and the pulse repetition frequencies are identical with $1.668 \mathrm{GHz}$, shown for the full span of the microwave spectrum analyzer (MSA) of $13 \mathrm{GHz}$ with a resolution bandwidth (RBW) of $30 \mathrm{kHz}$ [Fig. 7.2(c)] and magnified around the first harmonic in a span of $10 \mathrm{MHz}$ with a RBW of $1 \mathrm{kHz}$ [Fig. 7.2(d)]. The goal of this experiment was not to obtain a new record performance of CPM VECSELs but to study the coherence of the two output beams and investigate if coherent beam combining is possible. Therefore, we did not optimize the CPM VECSEL for short pulse durations but used a very stable configuration at room temperature that delivered reproducible parameters over weeks of operation. 
a)

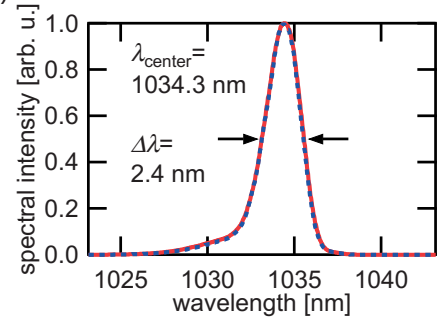

C)

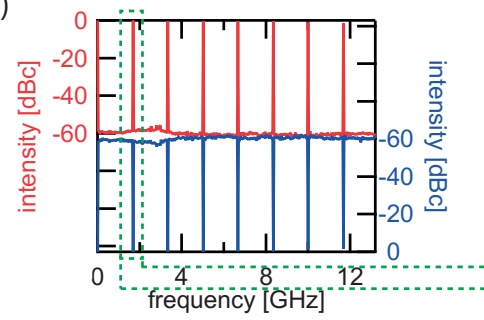

b)

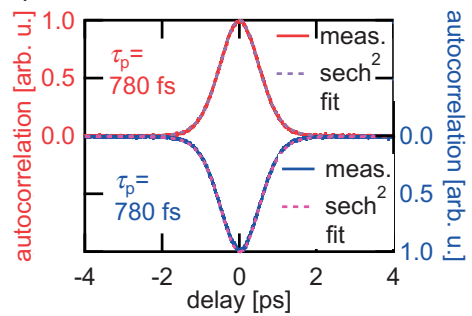

d)

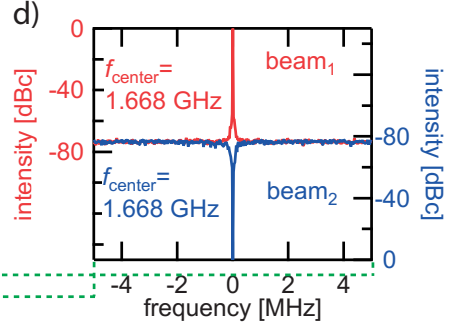

Figure 7.2: CPM VECSEL modelocking results for the two output beams: beam 1 (red) and beam 2 (blue). a) The optical spectrum is identical for both beams. b) Second harmonic autocorrelations show for each beam a pulse duration of $780 \mathrm{fs}$. c) Microwave spectrum measured over the full span of the microwave spectrum analyzer with a resolution bandwidth (RBW) of $30 \mathrm{kHz}$. d) Microwave spectrum zoomed in around the first harmonic with a span of $10 \mathrm{MHz}$ and a RBW of $1 \mathrm{kHz}$ confirms that both beams have the same pulse repetition frequency.

\subsubsection{Pulse repetition frequency analysis}

In CPM operation both pulses collide in the SESAM. Constructive interference of the two pulses increases the saturation of the SESAM which is energetically favorable. Therefore we expect that CPM locks the pulse repetition rates of the two counter-propagating beams. We recently observed similar intrinsic locking in case of a dual-comb modelocked SDL with sufficient spatial and temporal overlap on the absorber [18]. However, the microwave spectrum measurement of the pulse repetition frequency [Fig. 7.2(d)] is not sufficient as proof for perfectly synchronized pulses, since small asynchronous timing jittering would not be resolved with an MSA measurement.

We actively stabilize the pulse repetition frequency of one of the two 
beams $\left(\right.$ beam $\left._{1}\right)$ by actively controlling the cavity roundtrip length. We then measure the phase noise of both beams with a signal source analyzer (SSA) [Fig. 7.3]. For this stabilization approach we detect the pulse repetition frequency of beam 1 with a photodetector and mix the signal with a low noise electronic reference set to the same frequency. The phase error of the mixed signal is fed to a phase-locked loop (PLL). We exchanged the half-inch output coupler with a 2-mm thick output coupler that we mount on a piezo actuator. By sending the correction voltage of the PLL to the piezo, the cavity length is actively changed to compensate for the phase error. With a second photodetector, we measure the phase noise of beam $_{1}$ out-of-loop with the SSA. A strong reduction of the phase noise of over $100 \mathrm{~dB}$ is observed from free-running to stabilized operation [Fig. 7.3]. We keep the stabilization of beam 1 active and measure the phase noise of beam $_{2}$. Also for the pulse repetition frequency of beam 2 the phase noise is reduced by the same amount [Fig. 7.3], confirming that both pulse repetition rates are fully locked.

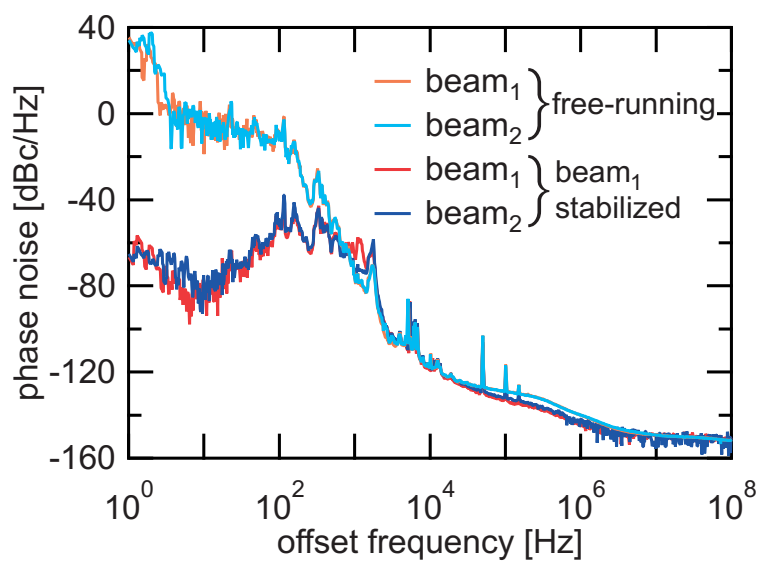

Figure 7.3: Phase noise measurements of the pulse repetition frequencies of the two output beams from the CPM VECSEL. If the pulse repetition frequency of beam is $_{1}$ actively stabilized by controlling the cavity roundtrip time, the noise is drastically reduced for both beams simultaneously, confirming that the two pulse repetition frequencies are locked with CPM. Note that it was not possible to obtain the same stabilization by controlling the pump power. 
In CPM the intrinsic lock of the pulse repetition rate is based on the colliding pulses on the saturable absorber which is a pulse-energy dependent and interferometric mechanism. Thus, stabilization via the cavity length is an independent feedback mechanism. To investigate further, we also try to stabilize the pulse repetition frequency of beam ${ }_{1}$ by modulating the current of the pump diode instead of the cavity length. All other parts of the detection and the PLL remain unchanged. In this configuration, it is not possible to lock the phase noise. We did try to stabilize the CPM laser via pump-power modulation under different operation parameters such as cooling temperatures, pump power levels and cavity lengths, but still no locking was achieved.

Previously without CPM we stabilized the pulse repetition frequency of a standard SESAM-modelocked VECSEL via both cavity length and pumppower control and obtained the same stabilization performance. However, the pump power modulation changes the pulse energy and therefore starts to affect the CPM. The effect is so strong that no active stabilization of the pulse repetition rate is possible with pump-power control any more.

\subsubsection{Carrier envelope offset (CEO) frequency analysis}

For coherent beam combining we need a fully locked frequency comb with both a locked pulse repetition frequency and a carrier envelope offset (CEO) frequency. For the following analysis of CEO frequencies, the pulse repetition frequency is not stabilized. Assuming the two CEO frequencies in each output beam are different but relatively stable with respect to each other, we should be able to detect the difference in CEO frequency as a beat signal on a photodetector. We obtain both a spatial and temporal overlap of the two output beams in the same polarization with a delay stage in the path of beam 2 before the spatial combination of the two beams with a 50:50 beam splitter (BS) [Fig. 7.4(a)]. The temporal overlap is confirmed by an autocorrelation measurement. However, no relative CEO beat frequency is visible in the microwave spectrum [Fig. 7.4(b)]. This means that either both CEO frequencies are identical or their relative noise is so high that the beat frequency stays below the noise floor of the MSA. To distinguish these two possibilities, we send beam 2 through an acousto-optic modulator (AOM) with a modulation frequency of $60 \mathrm{MHz}$ [Fig. 7.4(c)]. 
We take the first diffraction order which introduces a frequency shift by $60 \mathrm{MHz}$ for the full frequency comb of beam 2 , resulting in an effective CEO frequency that is $60 \mathrm{MHz}$ higher than before. If both CEO frequencies were the same, a relative beat signal should become visible at $60 \mathrm{MHz}$.
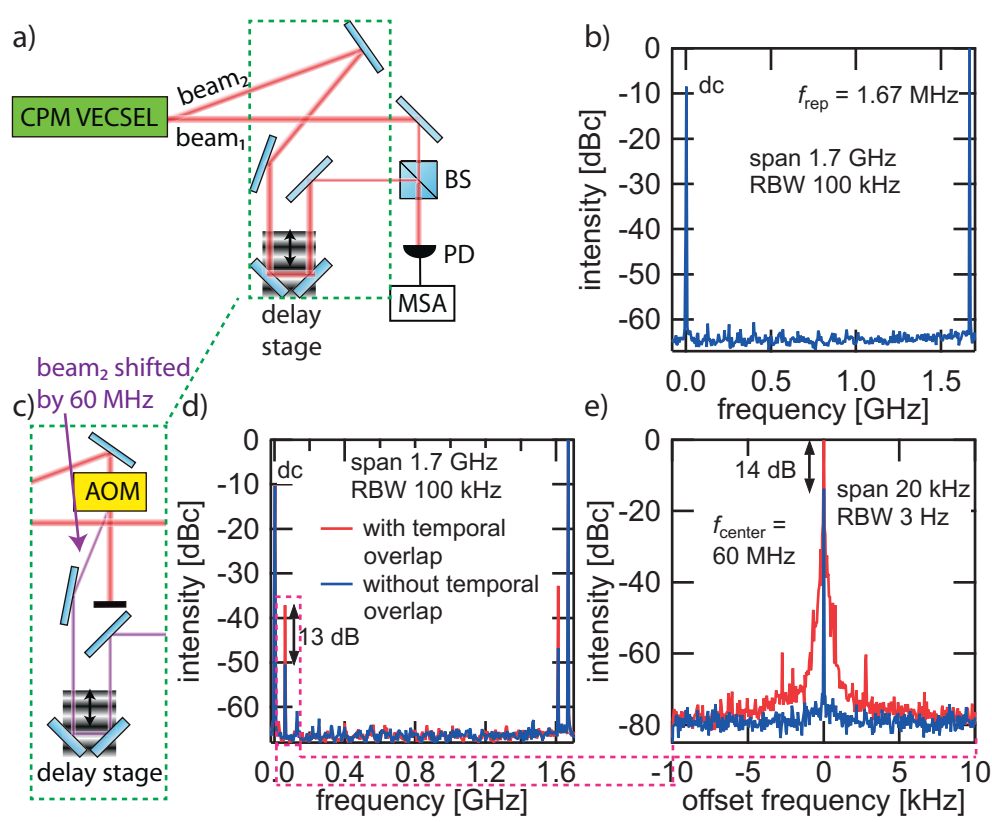

Figure 7.4: Locked carrier envelope offset (CEO) frequencies of the two output beams from the CPM VECSEL: a) Setup to combine both beams with temporal overlap. BS, beams splitter; PD, photodetector; MSA microwave spectrum analyzer. b) The microwave spectrum shows no relative $\mathrm{CEO}$ beat frequency, maybe indicating that both CEO frequencies are identical. c) This is confirmed by shifting the frequency of beam 2 with an acousto-optic modulator (AOM) by $60 \mathrm{MHz}$ and again combining the two beams with temporal overlap. d) CEO beat frequency is detected in the microwave spectrum at $60 \mathrm{MHz}$ (red line) which is stronger than the peak coming from the radio frequency driver of the AOM that is also present without temporal overlap (blue line). e) Magnification of the microwave spectrum around the $\mathrm{CEO}$ beat frequency.

We combine the frequency-shifted beam 2 with beam 1 and measure the microwave spectrum. Without temporal overlap, there is already a signal 
a)

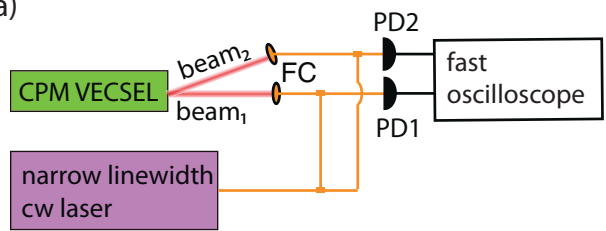

b)

CEO fluctuation

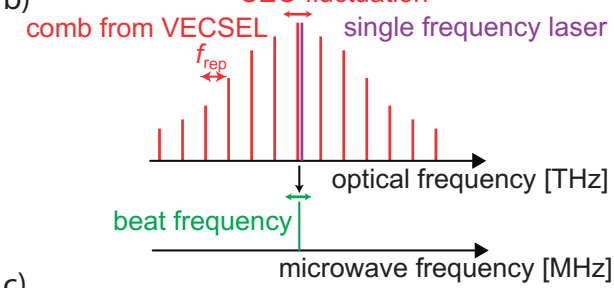

c)

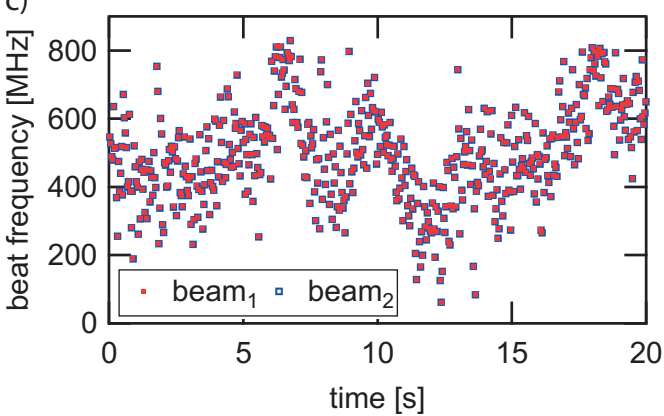

Figure 7.5: Long-term CEO frequency fluctuations of the two output beams of the CPM VECSEL: a) Setup to detect the CEO frequency fluctuations of both beams simultaneously, by beating each comb with a narrow linewidth laser. FC, fiber coupling; PD photodetector. b) The interference between each comb of the VECSEL and the narrow linewidth laser generate a beat frequency, whose fluctuations are equal to the fluctuations of the CEO frequency of the VECSEL comb. Since we only want to observe, if both CEO frequencies fluctuate identically, we can ignore the fluctuations due to the pulse repetition frequency since we already confirmed that they are intrinsically locked with CPM. c) The perfectly synchronized fluctuations of the two beat frequencies are measured over $20 \mathrm{~s}$ with a gate time of $5 \mu \mathrm{s}$. Due to the calculation time of the Fourier transformation, the time between two measurements is $37 \mathrm{~ms}$. 
detectable at $60 \mathrm{MHz}$. This signal comes from the strong radio frequency signal generated by the driver of the AOM and has a linewidth below the resolution of our MSA [blue line in Fig. 7.4(d), (e)]. When both beams are combined with temporal overlap, the beat signal at $60 \mathrm{MHz}$ increases by $13 \mathrm{~dB}$ [red line in Fig. 7.4(d)]. The magnification of the CEO beat signal with a span of $20 \mathrm{kHz}$ and a resolution bandwidth of $3 \mathrm{~Hz}$ shows a coherent peak of $23 \mathrm{~dB}$ on top of a noise pedestal of roughly $1 \mathrm{kHz}$ width [Fig. 7.4(e)]. The residual noise in the $1 \mathrm{kHz}$ range is not caused by a difference in CEO of the two beams but is a result of the phase noise accumulated on the different paths of the beams, especially due to the mechanically not perfectly stable delay stage. We confirm the origin of the noise by splitting beam ${ }_{1}$ with a beam splitter, sending one part through the AOM and the delay stage and then recombine it with the other part. The measured CEO beating shows the same noise pedestal, even though we beat the CEO frequency of beam 1 with itself, shifted by $60 \mathrm{MHz}$.

The measurement confirms that both CEO frequencies are identical, at least on a shorter time scale. To finally prove that they are perfectly locked also on a long time scale, we measure the CEO frequency of each beam simultaneously. This is realized by beating both beams with a fiber-coupled single frequency laser (Toptica DLC CTL 1050). The wavelength of the single frequency laser is tuned to the center wavelength of the two beams and combined with each beam using fiber combiners [Fig. 7.5(a)]. Both beat signals are recorded simultaneously with a photodetector and a fast oscilloscope with $1 \mathrm{GHz}$ bandwidth. The Fourier transformation of each time signal gives a frequency peak that fluctuates with the change of the CEO frequency of the corresponding beam [Fig. 7.5(b)]. Fluctuations of the pulse repetition frequency can be ignored since we have already confirmed in Section 7.1.3 that they are locked for both beams with CPM. We measure simultaneously the frequency fluctuations of the CEO frequency of each beam with a gate time of $5 \mu$ s [Fig. 7.5(c)]. The measurement shows clearly that both beat frequencies and therefore both $\mathrm{CEO}$ frequencies fluctuate in perfect synchronization. The large drifts of the CEO frequencies can be explained be the absence of any housing or shielding for the laser setup. 


\subsubsection{Coherent beam combining}

Since we have established that both beams of the CPM VECSEL have the same pulse repetition frequency and the same CEO frequency, all criteria for successful coherent beam combing of the two output beams are fulfilled. We test the coherent combination of the two beams in a peak-power sensitive second harmonic generation (SHG) experiment. Both beams are combined with a beam splitter (BS) and the temporal overlap is controlled with the delay stage in one of the beam paths [Fig. 7.6(a)]. The beams are then focused into a lithium triborate (LBO) crystal of $20 \mathrm{~mm}$ length that was temperature stabilized at $184^{\circ} \mathrm{C}$ for non-critical phase matching. A dichroic mirror is used to separate the near infrared light at $1034 \mathrm{~nm}$ and the generated green light at $517 \mathrm{~nm}$. The green light is measured with a photodetector and a fast oscilloscope.

If one of the beams is blocked, only half of the power of the other beam will go through the 50:50 BS and generate green [case I and II in Fig. 7.6(b)]. If both beams reach the BS but without temporal overlap, no interference occurs and half of the power of each beam will reach the crystal (case III). The power of the SHG signal scales with the square of the infrared pulse peak power. Therefore, without coherent combination the green signal in case III will be just the sum of the signals of case I and case II. The situation drastically changes for case IV in [Fig. 7.6]: If the pulses of both beams arrive at the same time on the beam splitter, they interfere constructively or destructively, depending on the phase difference of the beams accumulated in their respective beam paths. For ideal constructive interference, all of the power of each beam will go through the BS and arrive simultaneously on the SHG crystal and the peak power will add up. This leads to an eight-fold stronger green generation compared to case III. Since the interference is phase sensitive, it varies from constructive to destructive within half a wavelength $(517 \mathrm{~nm})$ of path-difference of the two beams. The experimental setup was on the open table without housing or special care for mechanically stable mounts. This explains why the SHG green power signal fluctuates strongly in case IV. However, it reaches a maximum of 7.7, normalized to case III, which is very close to the ideal maximum of 8 . The small difference to the ideal case can be explained by the fact that the power per beam reaching the BS was not identical 


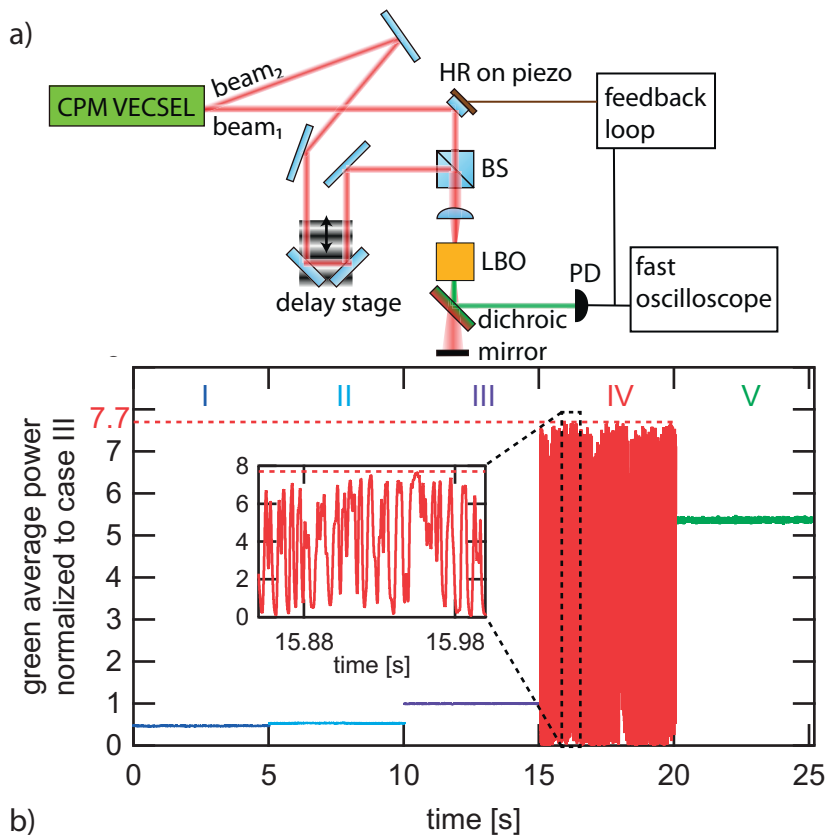

Figure 7.6: Proof-of-principle experiment for coherent beam combining of the two output beams from the CPM VECSEL. a) Both beams are coherently combined and focused into a 20-mm long lithium triborate (LBO) crystal to generate green light through second harmonic generation. PD, photodetector; BS, beam splitter. $b$ ) The generated green light is measured with a PD and a fast oscilloscope for 5 different cases: (I): beam ${ }_{2}$ is blocked and only half of the power of beam ${ }_{1}$ arrives at the LBO crystal. (II): beam 1 is blocked and only half of the power of beam 2 arrives at the crystal. (III): half of the power of both beams arrive at the crystal without temporal overlap which is simply a incoherent superposition of the two intensities. (IV): both beams are coherently combined and interfere constructive and destructive. (V): the coherently combined signal is stabilized by changing the path-length difference with a with a feedback loop and a piezo actuator. 
and by the imperfection of the BS. The oscillation-periods of the green signal are mostly above $1 \mathrm{~ms}$. This is confirmed by measuring the intensity noise of the SHG signal by taking the Fourier transformation of the signal in time [Fig. 7.7]. The noise drops to the noise floor in case IV above $\approx 1 \mathrm{kHz}$, which is a typical value for mechanical noise and air disturbances. This also corresponds to the noise pedestal for the relative CEO frequency measurement [Fig. 7.4(e)], since the origin of the noise is the same.

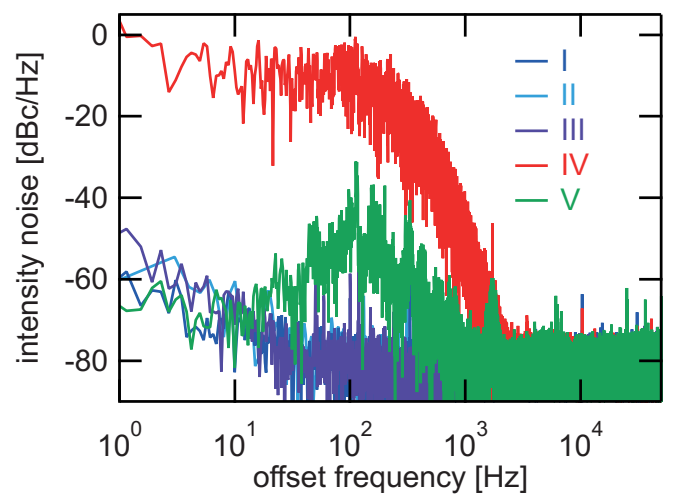

Figure 7.7: Intensity noise of the second harmonic generation (SHG), calculated via Fourier transformation from the time-dependent power signal measured in [Figure $7.6(\mathrm{~b})]$ for the five different cases. The mechanical fluctuations changing the beam path difference in case IV show a bandwidth of $\approx 1 \mathrm{kHz}$ before reaching the noise floor. They can be stabilized with a feedback loop and a piezo (case V).

The slow SHG power fluctuations with a bandwidth of $1 \mathrm{kHz}$ can be compensated with a mirror mounted on a piezo actuator. The voltage signal of the green light in case IV is compared to a fixed voltage from a power supply. The error signal is fed to the same PLL used in section 7.1.3 and the correction voltage is send to a piezo actuator to control the path length of beam 1 [Fig. 7.6(a)]. With this method, we achieved a stabilized second harmonic signal at a level 5.3 times higher (case V) than in case III. With this simple stabilization method, it is not possible to stabilize the signal to the maximum level, since the error signal must be on the slope of the voltage signal. The intend is here to show that the coherently combined 
signal can be stabilized with little effort even in a setup not optimized for low-noise operation. With a more advanced detection scheme such as a lock-in amplification method [19] or the Hänsch-Couillaud method using a polarizing beam splitter [20], it is also possible to stabilize the coherently combined signal to the maximum value.

\subsubsection{Conclusion and outlook}

We have realized colliding pulse modelocking (CPM) of a VECSELSESAM ring cavity. Our measurements show that both output beams have both identical pulse repetition frequencies and CEO frequencies. As expected from CPM we could only actively stabilize the pulse repetition rates of the two output beams with feedback over cavity length control and not with pump-power control. We can conclude that coherent beam combining of the two output beams is possible for further power scaling. We have demonstrated coherent beam combining in a proof-of-principle experiment with second harmonic generation.

For the current noise analysis experiments we did not optimize the VECSEL and the ring cavity for new world-record performance in terms of pulse duration and peak power. However, previously sub-200 fs pulses have been generated in such a CPM cavity configuration [7]. A coherent combination of the two beams has the potential to lead to a peak power of more than $1 \mathrm{~kW}$, which should be sufficient to enable coherent supercontinuum generation in silicon nitride waveguides [16] and generate a self-referenced octave-spanning optical frequency comb from such a CPM VECSEL without any external pulse amplification. Other applications may also benefit from two output beams with identical frequency comb properties.

Funding. Swiss Confederation Program Nano-Tera.ch, which was scientifically evaluated by the Swiss National Science Foundation (SNSF).

Acknowledgement The authors acknowledge support of the technology and cleanroom facility FIRST of ETH Zurich for advanced micro-and nanotechnology. 


\subsubsection{References}

1. R. L. Fork, B. I. Greene, and C. V. Shank, "Generation of optical pulses shorter than 0.1 ps by colliding pulse modelocking," Appl. Phys. Lett. 38, 617-619 (1981).

2. G. H. C. New, "Modelocking of quasi-continuous lasers," Opt. Commun. 6, 188-192 (1972).

3. J. A. Valdmanis and R. L. Fork, "Design Considerations for a Femtosecond Pulse Laser Balancing Self Phase Modulation, Group Velocity Dispersion, Saturable Absorption, and Saturable Gain," IEEE J. Quantum Electron. 22, 112-118 (1986).

4. Y. K. Chen, M. C. Wu, T. Tanbun-Ek, R. A. Logan, and M. A. Chin, "Subpicosecond monolithic colliding-pulse modelocked multiple quantum well lasers," Appl. Phys. Lett. 58, 1253-1255 (1991).

5. Y.-K. Chen and M. C. Wu, "Monolithic Colliding Pulse Modelocked Quantum Well Lasers," IEEE J. Quantum Electron. 28, 2176-2185 (1992).

6. A. Laurain, D. Marah, R. Rockmore, J. McInerney, J. Hader, A. R. Perez, W. Stolz, and J. V. Moloney, "Colliding pulse mode locking of vertical-externalcavity surface-emitting laser," Optica 3, 781-784 (2016).

7. A. Laurain, R. Rockmore, H. T. Chan, J. Hader, S. W. Koch, A. R. Perez, W. Stolz, and J. V. Moloney, "Pulse interactions in a colliding pulse mode-locked vertical external cavity surface emitting laser," J. Opt. Soc. Am. B, 34, 329-337 (2017).

8. R. L. Fork, B. I. Greene, and C. V. Shank, "Generation of optical pulses shorter than 0.1 ps by colliding pulse modelocking," Appl. Phys. Lett. 38, 617-619 (1981).

9. G. H. C. New, "Modelocking of quasi-continuous lasers, "Opt. Commun. 6, 188-192 (1972).

10. J. A. Valdmanis and R. L. Fork, "Design Considerations for a Femtosecond Pulse Laser Balancing Self Phase Modulation, Group Ve- 
locity Dispersion, Saturable Absorption, and Saturable Gain,“ IEEE J. Quantum Electron. 22, 112-118 (1986).

11. Y. K. Chen, M. C. Wu, T. Tanbun-Ek, R. A. Logan, and M. A. Chin, "Subpicosecond monolithic colliding-pulse modelocked multiple quantum well lasers," Appl. Phys. Lett. 58, 1253-1255 (1991).

12. Y.-K. Chen and M. C. Wu, "Monolithic Colliding Pulse Modelocked Quantum Well Lasers," IEEE J. Quantum Electron. 28, 2176-2185 (1992).

13. A. Laurain, D. Marah, R. Rockmore, J. McInerney, J. Hader, A. R. Perez, W. Stolz, and J. V. Moloney, "Colliding pulse mode locking of vertical-externalcavity surface-emitting laser," Optica 3, 781-784 (2016).

14. A. Laurain, R. Rockmore, H. T. Chan, J. Hader, S. W. Koch, A. R. Perez, W. Stolz, and J. V. Moloney, "Pulse interactions in a colliding pulse mode-locked vertical external cavity surface emitting laser," J. Opt. Soc. Am. B, 34, 329-337 (2017).

15. B. W. Tilma, M. Mangold, C. A. Zaugg, S. M. Link, D. Waldburger, A. Klenner, A. S. Mayer, E. Gini, M. Golling, and U. Keller, "Recent advances in ultrafast semiconductor disk lasers," Light Sci Appl 4, e310- (2015).

16. M. A. Gaafar, A. Rahimi-Iman, K. A. Fedorova, W. Stolz, E. U. Rafailov, and M. Koch, "Mode-locked semiconductor disk lasers," Adv. Opt. Photonics 8, 370-400 (2016).

17. D. Waldburger, S. M. Link, M. Mangold, C. G. E. Alfieri, E. Gini, M. Golling, B. W. Tilma, and U. Keller, "High-power 100 fs semiconductor disk lasers," Optica 3, 844-852 (2016).

18. D. J. H. C. Maas, A.-R. Bellancourt, B. Rudin, M. Golling, H. J. Unold, T. Südmeyer, and U. Keller, "Vertical integration of ultrafast semiconductor lasers," Appl. Phys. B 88, 493-497 (2007).

19. C. G. E. Alfieri, D. Waldburger, S. M. Link, E. Gini, M. Golling, G. Eisenstein, and U. Keller, "Optical efficiency and gain dynamics of modelocked semiconductor disk lasers," Opt. Express 25, 6402-6420 (2017). 
20. M. Mangold, S. M. Link, A. Klenner, C. A. Zaugg, M. Golling, B. W. Tilma, and U. Keller, "Amplitude noise and timing jitter characterization of a high-power mode-locked integrated external-cavity surface emitting laser," IEEE Photon. J. 6, 1-9 (2014).

21. S. M. Link, D. J. H. C. Maas, D. Waldburger, and U. Keller, "Dualcomb spectroscopy of water vapor with a free-running semiconductor disk laser,“ Science 356, 1164-1168 (2017).

22. F. F. Voigt, F. Emaury, P. Bethge, D. Waldburger, S. M. Link, S. Carta, A. v. d. Bourg, F. Helmchen, and U. Keller, "Multiphoton in vivo imaging with a femtosecond semiconductor disk laser," Biomed. Opt. Express 8, 3213-3231 (2017).

23. A. Klenner, A. S. Mayer, A. R. Johnson, K. Luke, M. R. E. Lamont, Y. Okawachi, M. Lipson, A. L. Gaeta, and U. Keller, "Gigahertz frequency comb offset stabilization based on supercontinuum generation in silicon nitride waveguides," Opt. Express. 24, 11043-11053 (2016).

24. H. R. Telle, G. Steinmeyer, A. E. Dunlop, J. Stenger, D. H. Sutter, and U. Keller, "Carrier-envelope offset phase control: A novel concept for absolute optical frequency measurement and ultrashort pulse generation,“ Appl. Phys. B 69, 327-332 (1999).

25. S. M. Link, A. Klenner, and U. Keller, "Dual-comb modelocked lasers: semiconductor saturable absorber mirror decouples noise stabilization," Opt. Express 24, 1889-1902 (2016).

26. T. M. Shay, V. Benham, J. T. Baker, A. D. Sanchez, D. Pilkington, and C. A. Lu, "Self-synchronous and self-referenced coherent beam combination for large optical arrays," IEEE J. Sel. Top. Quantum Electron. 13, 480 (2007).

27. T. W. Hänsch and B. Couillaud, "Laser frequency stabilization by polarization spectroscopy of a reflecting reference cavity," Opt. Commun. 35, 441-444 (1980). 


\section{Chapter 8}

\section{Conclusion and outlook}

Within this thesis, the MIXSEL technology has been developed from a single modelocked laser to a compact dual-comb laser that can be used in free-running operation to perform dual-comb gas spectroscopy.

The foundation for the use of MIXSELs for spectroscopic measurements was the thorough noise analysis of a single MIXSEL. The characterization revealed that these lasers have an excellent noise performance with recordlow timing-jitter values [32].

The key idea of this project was the invention of the single-cavity dualcomb concept: the intracavity birefringent crystal in the simple linear cavity of the MIXSEL enabled the simultaneous generation of two modelocked beams from a single laser cavity [35].

The interplay between the two beams in one cavity and their noise correlation was studied in detail. Within this analysis, it was also demonstrated that the dual-comb concept can be applied not only to semiconductor lasers but also to diode-pumped solid-state lasers with the presentation of a dual-comb SESAM-modelocked Nd:YAG laser [36].

It was possible to use the dual-comb MIXSEL in free-running operation to perform a first gas spectroscopy measurement of water vapor, due to the high mutual short-term coherence of the two beams sharing the same cavity. The stabilization techniques used in the previous noise analysis projects enabled a microwave-only stabilization of the down-converted 
dual-comb, allowing also for measurements with long integration times [49].

Since the main limitation of the first dual-comb MIXSEL presentation was the limited optical bandwidth due to the rather long picosecond pulses, special attention was paid to reduce the pulse duration of SDLs, resulting in the first sub-100 fs SDL with an optical bandwidth of $17 \mathrm{~nm}$ FWHM [21].

Finally, the knowledge about different noise characterization methods obtained during the work of this thesis was used to show for the first time that the $\mathrm{CEO}$ frequencies and the pulse repetition rates of the two counterpropagating beams in a CPM VECSEL are locked and that they can be coherently combined for power scaling [53].

The field of dual-comb spectroscopy has gained a lot of attention and is very rapidly evolving, as can be seen by the exponentially increasing number of publications and citations in this field (Fig. 8.1).
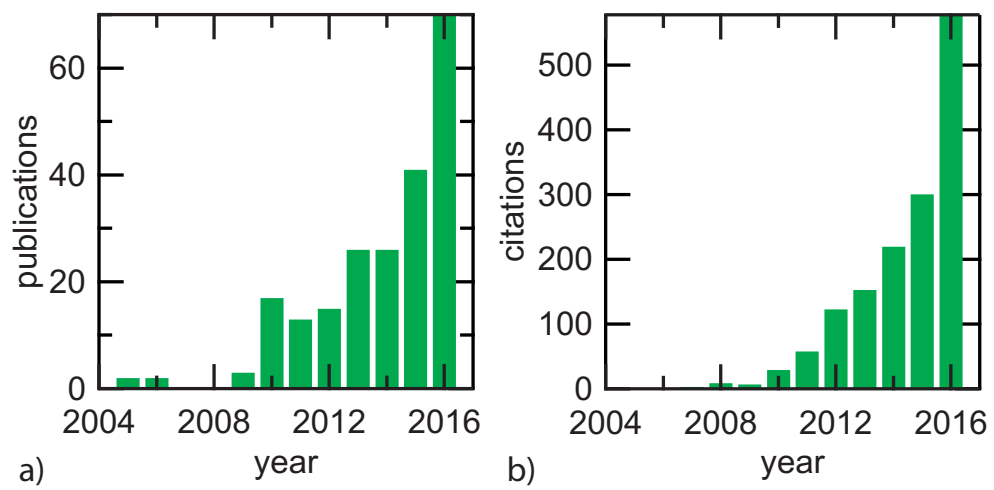

Figure 8.1: Increase of the number of a) publications and b) citations over the last years containing the key words "dual-comb" or "multi-heterodyne". The data is collected from "Web of Science" [54].

The dual-comb MIXSEL represents a very compelling, simple, compact, and cost-efficient source that could pave the way for wide-spread indus- 
trial dual-comb applications. In a next step, the new MIXSEL structures with their improved design, enabling femtosecond operation with several nanometers of optical bandwidth around a center wavelength of $1030 \mathrm{~nm}$ [27] need to be tested in the dual-comb configuration. Preliminary results in our laboratory already showed dual-comb operation with $10 \mathrm{~nm}$ FWHM optical bandwidth, which is a very promising step forward. This will allow for dual-comb spectroscopy on acetylene, an impurity occurring in the production of ethylene, which is a petrochemical produced on a large industrial scale. As a further outlook, new wavelength regimes need to be covered with the dual-comb MIXSEL.

The visible to ultra-violet regime is a spectroscopically very interesting region, since many molecules contain electronic transitions within this region. It should be straight forward to access this spectroscopy domain with the dual-comb MIXSEL technology via frequency doubling. Due to the relatively high average output power of SDLs, extra-cavity secondharmonic generation is possible. On the other side of the spectrum, the mid-infrared region is considered as the "fingerprint" region, since numerous molecules display strong absorption features in this region due to ro-vibrational transitions. To reach into the mid-infrared region with MIXSELs, different approaches could be pursued. Nonlinear broadening and difference frequency generation could be used to shift existing lasers into the mid-infrared. Since the emission wavelength of the semiconductor gain medium depends on its energy band-gap, it is also possible to tailor the wavelength with band-gap engineering. New SDLs based around $\mathrm{GaSb} / \mathrm{GaInAsSb}$ quantum wells could increase the emission wavelength to $3 \mu \mathrm{m}$. However, this requires a strong effort on the semiconductor growth, and different aspects such as thermal heat management and Auger-losses will pose new challenges.

The dual-comb MIXSEL represents a new class of compact and costefficient low-noise dual-comb lasers. The different chapters of this thesis retraced its development from the first conceptual idea to the experimental demonstration of its applicability for gas spectroscopy. There are still many new and exciting aspects to explore with this technology, but the presented results already show the very promising potential and the big impact that this laser could have. 



\section{Literature}

[1] T. H. Maiman, "Stimulated Optical Radiation in Ruby", Nature 187 (1960), 493-494

[2] T. W. Hänsch, "Nobel Lecture: Passion for precision", Reviews of Modern Physics 78 (2006), 1297-1309

[3] S. Schiller, "Spectrometry with frequency combs", Opt. Lett. 27 (2002), 766-768

[4] F. Keilmann, C. Gohle, R. Holzwarth, "Time-domain mid-infrared frequencycomb spectrometer", Opt. Lett. 29 (2004), 1542-1544

[5] I. Coddington, N. Newbury, W. Swann, "Dual-comb spectroscopy", Optica 3 (2016), 414-426

[6] H. R. Telle, G. Steinmeyer, A. E. Dunlop, J. Stenger, D. H. Sutter, U. Keller, "Carrier-envelope offset phase control: A novel concept for absolute optical frequency measurement and ultrashort pulse generation", Appl. Phys. B 69 (1999), 327-332

[7] R. N. Hall, G. E. Fenner, J. D. Kingsley, T. J. Soltys, R. O. Carlson, “Coherent Light Emission From GaAs Junctions", Physical Review Letters 9 (1962), 366

[8] H. Soda, K. Iga, C. Kitahara, Y. Suematsu, "GaInAsP-InP surface emitting injection-lasers", Japanese Journal of Applied Physics 18 (1979), 2329-2330

[9] M. A. Hadley, G. C. Wilson, K. Y. Lau, J. S. Smith, "High single transversemode output from external-cavity surface-emitting laser diodes", Applied Physics Letters 63 (1993), 1607-1609

[10] M. Kuznetsov, F. Hakimi, R. Sprague, A. Mooradian, "High-power ( $>0.5-\mathrm{W}$ $\mathrm{CW})$ diode-pumped vertical-external-cavity surface-emitting semiconductor lasers with circular TEM 00 beams", IEEE Photonics Technology Letters 9 (1997), 1063-1065

[11] U. Keller, A. C. Tropper, "Passively modelocked surface-emitting semiconductor lasers", Phys. Rep. 429 (2006), 67-120 
[12] N. Schulz, J. M. Hopkins, M. Rattunde, D. Burns, J. Wagner, "High-brightness longwavelength semiconductor disk lasers", Laser Photon. Rev. 2 (2008), 160181

[13] M. Guina, A. Härkönen, V.-M. Korpijärvi, T. Leinonen, S. Suomalainen, "Semiconductor disk lasers: recent advances in generation of yellow-orange and mid-IR radiation", Adv. Opt. Technol. 2012 (2012), 19

[14] B. W. Tilma, M. Mangold, C. A. Zaugg, S. M. Link, D. Waldburger, A. Klenner, A. S. Mayer, E. Gini, M. Golling, U. Keller, "Recent advances in ultrafast semiconductor disk lasers", Light Sci Appl 4 (2015), e310-

[15] A. Rahimi-Iman, "Recent advances in VECSELs", J. Opt. 18 (2016), 093003

[16] M. A. Gaafar, A. Rahimi-Iman, K. A. Fedorova, W. Stolz, E. U. Rafailov, M. Koch, "Mode-locked semiconductor disk lasers", Adv. Opt. Photonics 8 (2016), 370-400

[17] F. Zhang, B. Heinen, M. Wichmann, C. Möller, B. Kunert, A. Rahimi-Iman, W. Stolz, M. Koch, “A 23-Watt single-frequency vertical-external-cavity surfaceemitting laser", Optics Express 22 (2014), 12817-12822

[18] B. Heinen, T. L. Wang, M. Sparenberg, A. Weber, B. Kunert, J. Hader, S. W. Koch, J. V. Moloney, M. Koch, W. Stolz, "106 W continuous-wave output power from vertical-external-cavity surface-emitting laser", Electronics Letters 48 (2012), 516-517

[19] S. Hoogland, S. Dhanjal, J. S. Roberts, A. C. Tropper, R. Häring, R. Paschotta, F. Morier-Genoud, U. Keller, "Passively mode-locked diode-pumped surfaceemitting semiconductor laser", IEEE Photon. Technol. Lett. 12 (2000), 11351138

[20] C. W. Baker, M. Scheller, A. Laurain, A. Ruiz-Perez, W. Stolz, S. Addamane, G. Balakrishnan, S. W. Koch, R. J. Jones, J. V. Moloney, "Multi-angle VECSEL cavities for dispersion control and peak-power scaling", IEEE Photon. Technol. Lett. 29 (2017), 326-329

[21] D. Waldburger, S. M. Link, M. Mangold, C. G. E. Alfieri, E. Gini, M. Golling, B. W. Tilma, U. Keller, "High-power 100 fs semiconductor disk lasers", Optica 3 (2016), 844-852

[22] C. Hönninger, R. Paschotta, F. Morier-Genoud, M. Moser, U. Keller, “Qswitching stability limits of continuous-wave passive mode locking", J. Opt. Soc. Am. B 16 (1999), 46-56

[23] C. A. Zaugg, A. Klenner, O. D. Sieber, M. Golling, B. W. Tilma, U. Keller, "Sub-100 MHz Passively Modelocked VECSEL", in "CLEO: 2013", San Jose, California (2013), OSA Technical Digest (online), page CW1G.6, optical Society of America

[24] M. Mangold, C. A. Zaugg, S. M. Link, M. Golling, B. W. Tilma, U. Keller, "Pulse repetition rate scaling from $5 \mathrm{GHz}$ to $100 \mathrm{GHz}$ with a high-power semiconductor disk laser", Optics Express 22 (2014), 6099-6107 
[25] D. J. H. C. Maas, A.-R. Bellancourt, B. Rudin, M. Golling, H. J. Unold, T. Südmeyer, U. Keller, "Vertical integration of ultrafast semiconductor lasers", Appl. Phys. B 88 (2007), 493-497

[26] B. Rudin, V. J. Wittwer, D. J. H. C. Maas, M. Hoffmann, O. D. Sieber, Y. Barbarin, M. Golling, T. Südmeyer, U. Keller, "High-power MIXSEL: an integrated ultrafast semiconductor laser with $6.4 \mathrm{~W}$ average power", Opt. Express 18 (2010), 27582-27588

[27] C. G. E. Alfieri, D. Waldburger, S. M. Link, E. Gini, M. Golling, G. Eisenstein, U. Keller, "Optical efficiency and gain dynamics of modelocked semiconductor disk lasers", Opt. Express 25 (2017), 6402-6420

[28] S. M. Link, B. W. Tilma, M. Mangold, C. A. Zaugg, A. Klenner, U. Keller, "Pulsed laser", International Patent, WO 2016/049787 A1 (filed September 31, 2014, issued April 7, 2016)

[29] D. von der Linde, "Characterization of the noise in continuously operating mode-locked lasers", Appl. Phys. B 39 (1986), 201-217

[30] V. J. Wittwer, "New frontiers of ultrafast semicondcutor disk lasers: High power MIXSELs and low noise VECSELs", Ph.D. thesis, ETH Zürich (2012)

[31] R. Paschotta, "Noise of mode-locked lasers. Part II: Timing jitter and other fluctuations", Appl. Phys. B 79 (2004), 163-173

[32] M. Mangold, S. M. Link, A. Klenner, C. A. Zaugg, M. Golling, B. W. Tilma, U. Keller, "Amplitude noise and timing jitter characterization of a high-power mode-locked integrated external-cavity surface emitting laser", IEEE Photonics Journal 6 (2014), 1-9

[33] A. Bartels, R. Cerna, C. Kistner, A. Thoma, F. Hudert, C. Janke, T. Dekorsy, “Ultrafast time-domain spectroscopy based on high-speed asynchronous optical sampling", Rev. Sci. Instrum. 78 (2007), 035107

[34] B. E. A. Saleh, M. C. Teich, Fundamentals of photonics, (Chapter 6), John Wiley and Sons, Inc. (1991)

[35] S. M. Link, A. Klenner, M. Mangold, C. A. Zaugg, M. Golling, B. W. Tilma, U. Keller, "Dual-comb modelocked laser", Opt. Express 23 (2015), 5521-5531

[36] S. M. Link, A. Klenner, U. Keller, “Dual-comb modelocked lasers: semiconductor saturable absorber mirror decouples noise stabilization", Opt. Express 24 (2016), 1889-1902

[37] B. Bernhardt, "Dual comb spectroscopy", Ph.D. thesis, Ludwig-MaximiliansUniversität (2011)

[38] G. Villares, A. Hugi, S. Blaser, J. Faist, "Dual-comb spectroscopy based on quantum-cascade-laser frequency combs", Nat. Commun. 5 (2014), 5192

[39] M.-G. Suh, Q.-F. Yang, K. Y. Yang, X. Yi, K. J. Vahala, “Microresonator soliton dual-comb spectroscopy", Science 354 (2016), 600-603 
[40] A. Hugi, G. Villares, S. Blaser, H. C. Liu, J. Faist, "Mid-infrared frequency comb based on a quantum cascade laser", Nature 492 (2012), 229-233

[41] G. Villares, J. Wolf, D. Kazakov, M. J. Süess, A. Hugi, M. Beck, J. Faist, “On-chip dual-comb based on quantum cascade laser frequency combs", Appl. Phys. Lett. 107 (2015), 251104

[42] J. Faist, G. Villares, G. Scalari, M. Rösch, C. Bonzon, A. Hugi, M. Beck, “Quantum cascade laser frequency combs", Nanophotonics 5 (2016)

[43] M. Rösch, G. Scalari, G. Villares, L. Bosco, M. Beck, J. Faist, “On-chip, selfdetected terahertz dual-comb source", Appl. Phys. Lett. 108 (2016)

[44] T. J. Kippenberg, R. Holzwarth, S. A. Diddams, "Microresonator-based optical frequency combs", Science 332 (2011), 555-559

[45] X. Yi, Q.-F. Yang, K. Y. Yang, K. Vahala, "Active capture and stabilization of temporal solitons in microresonators", Opt. Lett. 41 (2016)

[46] N. G. Pavlov, G. Lihachev, S. Koptyaev, E. Lucas, M. Karpov, I. A. Kondratiev, N. M.and Bilenko, M. L. Kippenberg, T. J.and Gorodetsky, "Soliton dual frequency combs in crystalline microresonators", Opt. Lett. 42 (2017), 514-517

[47] X. Zhao, G. Hu, B. Zhao, C. Li, Y. Pan, Y. Liu, T. Yasui, Z. Zheng, "Picometerresolution dual-comb spectroscopy with a free-running fiber laser", Opt. Express 24 (2016), 21833-21845

[48] G. Millot, S. Pitois, M. Yan, T. Hovhannisyan, A. Bendahmane, T. W. Hänsch, N. Picqué, "Frequency-agile dual-comb spectroscopy", Nat. Photon. 10 (2016), 27-30

[49] S. M. Link, D. J. H. C. Maas, D. Waldburger, U. Keller, "Dual-comb spectroscopy of water vapor with a free-running semiconductor disk laser", Science 356 (2017), 1164-1168

[50] O. D. Sieber, M. Hoffmann, V. J. Wittwer, M. Mangold, M. Golling, B. W. Tilma, T. Südmeyer, U. Keller, "Experimentally verified pulse formation model for high-power femtosecond VECSELs", Applied Physics B 113 (2013), 133-145

[51] A. Laurain, D. Marah, R. Rockmore, J. McInerney, J. Hader, A. R. Perez, W. Stolz, J. V. Moloney, "Colliding pulse mode locking of verticalexternalcavity surface-emitting laser", Optica 3 (2016), 781-784

[52] A. Laurain, R. Rockmore, H. T. Chan, J. Hader, S. W. Koch, A. R. Perez, W. Stolz, J. V. Moloney, "Pulse interactions in a colliding pulse mode-locked vertical external cavity surface emitting laser", J. Opt. Soc. Am. B, 34 (2017), 329-337

[53] S. M. Link, D. Waldburger, C. G. E. Alfieri, M. Golling, U. Keller, "Coherent beam combining and noise analysis of a colliding pulse modelocked VECSEL", Opt. Express 25 (2017), 19281-19290

[54] https://apps.webofknowledge.com 


\title{
Curriculum Vitæ
}

\author{
Name Sandro Michael Link \\ Date of Birth September $23^{\text {th }}, 1987$ \\ Citizenship Germany
}

\section{Education}

PhD studies in the Ultrafast Laser Physics group of

2013 - 2017 Prof. U. Keller, Institute for Quantum Electronics, Department of Physics, ETH Zürich, Zürich, Switzerland

2012 - 2013 Master studies in Physics, ETH Zürich, Zürich, Switzerland

2008 - 2012 Bachelor studies in Physics, ETH Zürich, Zürich, Switzerland

1998 - 2007 Abitur, Alexander von Humboldt Gymnasium, Konstanz, Germany

\section{Research and Professional Experience}

PhD studies in the Ultrafast Laser Physics group of

since 2013 Prof. U. Keller, Institute for Quantum Electronics, Department of Physics, ETH Zürich, Zürich, Switzerland. Dual-Comb Modelocked Lasers.

Master thesis in the Ultrafast Laser Physics group of

Mar. - Jul. 2013 Prof. U. Keller, Institute for Quantum Electronics, Department of Physics, ETH Zürich, Zürich, Switzerland. Noise Characterization of a Prototype Integrated MIXSEL

Semester thesis in the Ultrafast Laser Physics group of Prof. U. Keller, Institute for Quantum Electronics,

Jun. 2012 Department of Physics, ETH Zürich, Zürich, Switzerland SESAM studies: Effect of Controlled Change of SESAM Temperature on Modulation Depth, Saturation Fluence, Damage Threshold and Recovery Time.

Sept. 2011 - Feb. Internship at greenTEG, Zürich, Switzerland. Development of 2012 an Automated Test Setup

2010 - 2012 Teaching assistant of the Department of Mathematics, ETH Zürich, Zürich, Switzerland.

Awards

Nov. 2016 Overall Best Student Presentation at Advanced Solid-State Lasers conference, Boston, USA

Oct. 2015 Outstanding Oral Presentation Award at Advanced Solid-State Lasers conference, Berlin, Germany

Jun. 2007 Award for outstanding overall performance in the Abitur 



\section{Acknowledgments}

I would like to thank and acknowledge all the people who supported me during my $\mathrm{PhD}$ and who made this thesis a successful endeavor and thanks to whom the last four years have been a very pleasant period for me.

Of course I would like to thank Prof. Dr. Ursula Keller for giving me the opportunity to pursue my PhD studies in her ULP group. The freedom and flexibility she gave me to work on new projects and her openness towards new ideas made the dual-comb MIXSEL possible. I am very grateful for all the instructive input and her believing in me.

Many thanks to Prof. Dr. Nathalie Picqué for accepting to be my coexaminer and for all the interesting discussions about spectroscopy. I am convinced that the future collaboration between her group and the ULP group will be very exciting and fruitful.

The collaboration with Dr. Deran Maas from ABB Switzerland was very pleasant and productive. I am thankful for all the useful input and the Matlab codes Deran gave to me.

The foundation for the success of this thesis was the great spirit and teamwork of the VECSEL and HIGHREP team and I would like to express my deep gratitude to all current and former team members, not only for the working time but also for the great time during conferences and trips: Dr. Mario Mangold for the excellent supervision of my Master thesis, Dr. Bauke Tilma for always leading the VECSEL team with patience and great support for the PhD students, Dr. Christian Zaugg for the great atmosphere in the lab and the office, Dr. Alexander Klenner for the perfect teamwork in the lab and all the knowledge about the "rfdomain", Dominik Waldburger and Cesare Alfieri for the discussions and all the projects that we worked on together efficiently and with fun, Jacob Nürnberg for continuing the dual-comb research, Aline Mayer for always providing a helping hand and for her dedication for all social group events and Dr. Florian Emaury for all his ideas and his enthusiasm. 
I would like to thank Dr. Matthias Golling and Dr. Emilio Gini for their effort on providing us continuously with new semiconductor structures and keeping the growth running.

The great atmosphere in the ULP group is one key aspect that enables all the excellent results and ideas from this group. I would like to thank all my current and former colleagues for the wonderful time during working hours but also during all the fun activities and for sharing great moments, exciting trips and many beers: Andreas Diebold and Ivan Graumann for sharing the office with me, it was always funny despite the extreme temperatures thanks to our lovely fridge, Dr. Chris Philips for the very informative and interesting discussions, Dr. Cinia Schriber for the supervision of my semester project, Nicolas Bigler for all the humorous conversations, Dr. André Ludwig, Dr. Benedikt Mayer, Dr. Cornelia Hofmann, Lamia Kasmi, Jannie Vos, Nadja Hartmann, Justas Pupeikis and Fabian Schläpfer for their great job as Partyministers, Dr. Robert Boge, Dr. Laura Cattaneo, Dr. Claudio Cirelli, Jaco Fuchs, Dr. Lukas Gallmann, Dr. Jens Herrmann, Dr. Sebastian Heuser, Stephan Hrisafov, Dr. Matteo Lucchini, Dr. Jochen Maurer, Luca Pedrelli, Dr. Mazyar Sabbar, Francesco Saltarelli, Dr. Clara Saraceno, Misha Volkov and Benjamin Willenberg.

I would like to thank Sandra Schmid for the perfect organization and the smooth administration of the group as well as the pleasant conversations about dogs.

Many mechanical parts of my projects were fabricated by the D-PHYS workshop led by Andi Stuker and Bernhard Morath and I would like to thank them for their precise and fast work. I am very thankful to Martin Gähwiler, Lukas Gautschi and the electronic laboratory for their expertise and advice on all kinds of electrical questions and the fabrication of several electrical devices.

For my time as responsible of the PECVD in FIRST I would like to thank Petra Burkard for the good teamwork and all her help, as well as Dr. Yargo Bonetti for the support of the machine.

I am deeply grateful to my parents Elke and Volker Link and my sister Helen Link for always supporting me. None of my achievements would have been possible without all your love and care.

My biggest gratitude belongs to my wonderful wife and soulmate Marion Link and our adorable son Joschua for always being there for me and making everyday unforgettable.

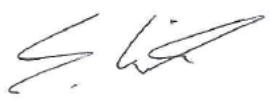

Zürich, 2017 